\title{
HOST-GUEST INTERACTIONS: FIRST-GENERATION FILIPINO IMMIGRANTS IN NEW ZEALAND AND THEIR VISITING FRIENDS AND RELATIVES (VFR) FROM THE PHILIPPINES
}

BY

ROBERT CHARLES GARCIA CAPISTRANO

\begin{abstract}
A thesis
submitted to the Victoria University of Wellington in fulfilment of the requirements for the degree of Doctor of Philosophy in Tourism Management
\end{abstract}

Victoria University of Wellington

2014 


\section{Abstract}

This thesis examines social interactions between Filipino immigrant-hosts residing in New Zealand and their visiting friends and relatives (VFRs) from the Philippines as guests. The growth of migration and immigrant communities globally has become a major source of tourists resulting from developing and extending relationships and kinship in the receiving country of the immigrant-hosts. Despite advances in VFR travel research, most studies focus on the VFR travellers and generally neglect the significance of the host as a factor in the overall travel experience. There is a need to examine host-guest relationships in the context of VFR travel research as travel and tourism have by and large neglected issues of sociality and how it is concerned with social relations. Similarly, there is an element of "othering" comprised in the host-guest relationship when the social interaction is a meeting of strangers. However, this study explores social interactions where the actors take on host and guest roles that are layered upon other elements of their pre-existing relationships. This research recognises that while the hosts and guests may share a similar cultural background, the social interactions under study take place in a different cultural setting.

The overall question that guides this research is: "How are social interactions between immigrant-hosts and their VFRs understood and interpreted by them?" Currently, there is a lack of conceptual and theoretical understanding of VFR travel and the hostguest phenomenon, as well as of the meanings and interpretations resulting from their social interactions. The underpinning paradigm for this thesis is hermeneutic phenomenology, which seeks to understand the truths derived from the experiences. This paradigm guides the study to derive an understanding of the social interactions and the meanings that immigrant-hosts and their guests attach to situations. A holistic approach was utilised to examine the social interactions of the immigrant-host and VFR relationship incorporating social exchange theory and the theory of emotional solidarity which will enable consideration of the various dimensions of social interaction. 
Individual and family/group interviews were conducted after the visit in order to provide a comprehensive approach and capture the range of interactions that occur between hosts and guests. The immigrant-host families and VFRs were interviewed after the post-visit phase in New Zealand and the Philippines, respectively. This study therefore incorporates multiple perspectives in studying VFR travel across time and space. Through thematic analysis and qualitative metasynthesis, the meanings provided by the hosts and guests to situations which occurred during the visit are analysed in order to give a voice to these groups.

The social interactions between immigrant-hosts and VFRs are dynamic, multidimensional and multi-faceted when examined from the multiple perspectives of the hosts and guests. The findings indicate that friendship and kinship appear to be special relationships to which people attach great importance, both personally and culturally, as friends and relatives provide a sense of identity and reaffirm social ties. This thesis contributes to current knowledge in interpreting the meanings of friendship and kinship in a cultural context and how it relates to VFR travel which may have an implication for both tourism and migration and on understanding the social interactions of immigrant-hosts living in their new homeland and their families and friends who visit them. 
This thesis is dedicated to the Filipino immigrant-hosts of New Zealand and their visiting loved ones from the Philippines. May succeeding generations of Filipinos in New Zealand continue to fuel VFR travel and inspire more research on other cultures. 


\section{Acknowledgements}

I would like to take this opportunity to express my gratitude to the people who have been instrumental in the successful completion of this thesis. Apart from my efforts, the success of any project depended largely on the encouragement and prayers of many others who I may have not mentioned in my thesis.

My acknowledgements begin at Victoria University of Wellington, the nexus of my work and study:

- I would like to show my deepest appreciation to my academic advisors, Dr. Adam Weaver and Prof. Douglas Pearce. I will be forever indebted to them and I will bring with me the wisdom that both have shared wherever I may go. I wish to thank them for their tremendous experience, guidance, and support during this sometimes difficult journey.

- Thank-you Dr. Ian Yeoman as he gave me a complimentary copy of his co-edited book on family tourism.

- My sincerest appreciation to Dr. Karen Smith for initially reviewing my thesis and to the examiners who have read the thesis in its entirety: Dr. Julia Albrecht, Dr. Heike Schänzel, and Dr. Elisa Backer.

- I am also very grateful to Dr. Sally Riad and Dr. Deborah Jones for being superb PhD Coordinators as well as to Megan Key, Luisa Acheson, and Sophia Lum for their administrative support.

This thesis would not have been possible without the financial assistance of the university including: the Victoria Doctoral Scholarship; Victoria Hardship Fund; Victoria Submission Scholarship; and funds from the School of Management to conduct interviews in the Philippines. Thank-you to Dr. Tina Cartmell and Dr. Jennifer Pilgrim, for looking after my overall wellbeing and I also wish to thank my colleagues in the PhD programme including: Ina Reichenberger, Tibor Zsirmik, Tuan Ngoc Le, Sandra Goh, Cesar Guala, Sarah Kimani, and Geoff Harrison for the smiles and words of encouragement. I wish them all success on their academic and professional journeys. 
Like VFR travel, my relationship with friends and relatives encompasses both time and space as my life, loved ones, and supporters are like the Filipino diaspora all over the world.

In New Zealand, I would like to thank:

- Pedro de Ocampo and Jude Doliente for hosting me during my stay in Auckland which allowed me to conduct interviews there and to obtain more hosting families from that city;

- Vicky Belegal, Ira Castro, and Rio Yonson for the laughter and humour that we share which kept our sanity intact while studying at Vic;

- Pola and Ging for the food and drinks that we shared and for accommodating me in their home during the final stage of my thesis (oral examination and revisions);

- Ka Uro and Mon Illana for allowing me to post the advertisement online in order to recruit my study participants;

- Dr. Maya Bernardo for being a good listener and a big sister to me; and

- The Philippine Embassy in Wellington for introducing me to the Filipino community during my first year in this city.

In the Philippines, I would like to extend thank-you to:

- my high school friend, Ariel Benipayo, for teaching me how to use Express Scribe, a software which facilitates transcription;

- Dr. Clarence Batan and his colleagues in the University of Santo Tomas for teaching me and allowing me to use the faculty room for the AtlasTi software tutorials;

- Rose Sontillanosa for the Philippine map and plotting the different locations for this research;

- Manang Amy, our nanny (domestic support in my ancestral home) for eight years of loyalty which is immeasurably appreciated;

- My dear uncle, Terry Capistrano for his financial assistance which allowed me to continue my studies, when the scholarships I have received had run out; and 
- Immigration New Zealand in Makati City for providing some statistical data relevant in this research.

In Canada, I am so thankful to:

- Liz Wilson, my former classmate in the Marine Affairs Programme (MAP), who pushed me to pursue a PhD and aspire and to excel at my academic passion and to stay committed to my goals;

- Becky Field of MAP-Dalhousie University, for keeping in touch with me even if I have embarked in a different academic discipline;

- Filipino immigrants of Halifax who have hosted me and for being true friends (including the families of Tita Cecille and Tito Sandy as well as Kuya Al and Ate Beth;

- Brad for sharing a wonderful time with your family during Christmas 2013 and our many overseas travels, involving VFR travel;

- My relatives in New Brunswick: Tita Susan, Tito Allan, Jan-jan, and Joshua.

In the United States, I thank my mother for her support and giving me the belief that I can survive the journey to earn a PhD. She has been my inspiration as she rises despite all adversities. I admire her for being an industrious and intelligent woman who studied federal and state accounting and taxation at a mature age and managed to succeed despite daunting obstacles in life.

While I may have chosen to take the "road less travelled" of the overseas scholar, there was a profound emptiness within me while I was separated from my homeland and that I felt most when I could not celebrate special occasions with family who were in another country. I especially missed sharing holidays and special moments with my family members in the Philippines, especially with my only sister Elaine and her daughter Eunice.

Most of all, I would like to thank the Filipino immigrant-host families in New Zealand and their respective visiting friends and relatives in the Philippines who willingly shared their precious time during the interview process. Aside from sharing their experiences of hosting or visiting New Zealand, they have at times provided me with 
accommodation, food, or transportation. Saying "thank you" is not nearly enough to express my sincere gratitude or utang na loob (debt of goodwill), in Philippine language. 


\section{Table of Contents}

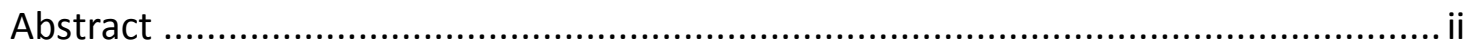

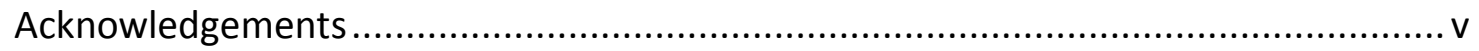

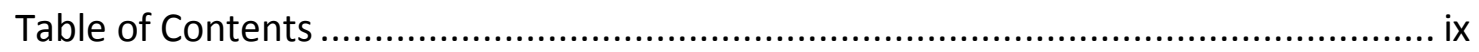

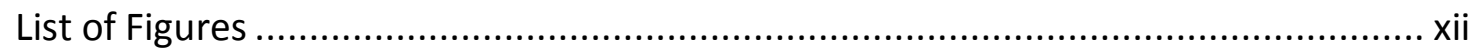

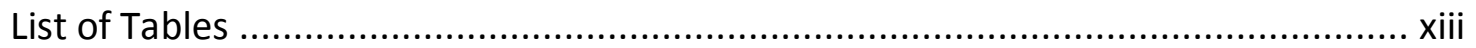

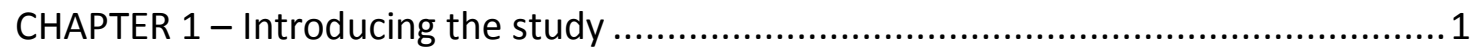

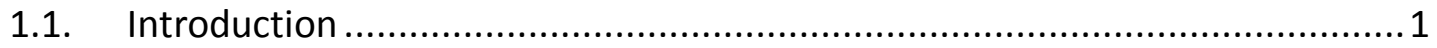

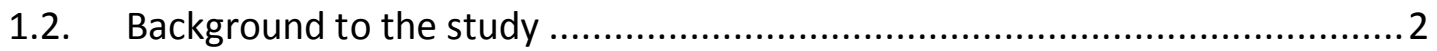

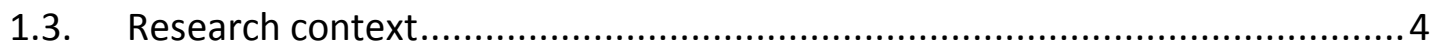

1.4. The research setting - New Zealand ................................................... 6

1.5. Overall objective and research questions .............................................. 9

1.6. Contribution of the research to wider knowledge ................................... 10

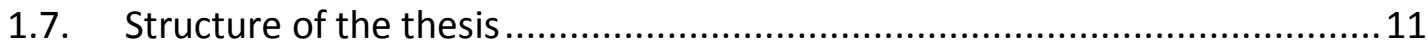

CHAPTER 2 - Literature review and conceptual framework ................................... 13

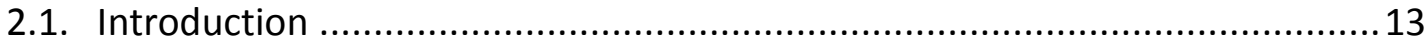

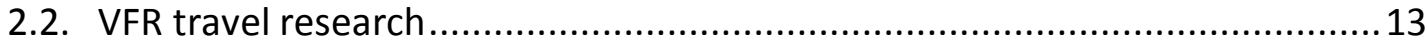

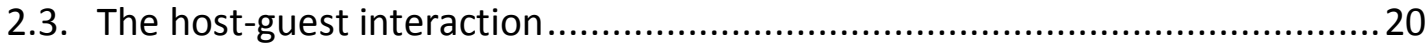

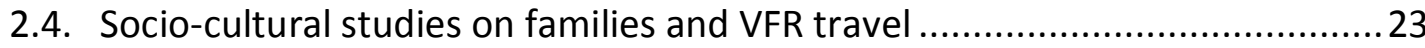

2.5. Friendships, social interactions, and VF travel research ..............................30

2.6. Towards a conceptual framework: a focus on Immigrant-VFR traveller

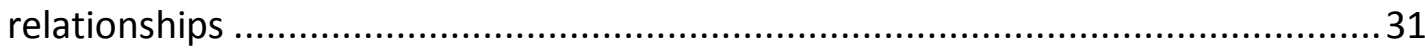

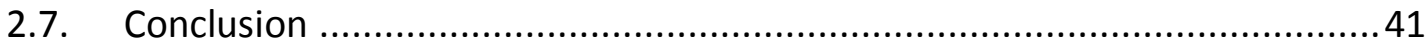

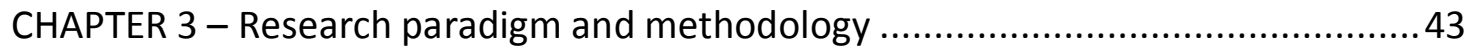

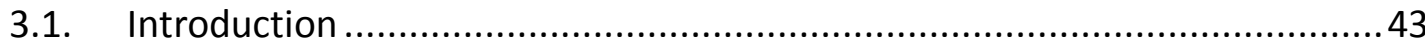

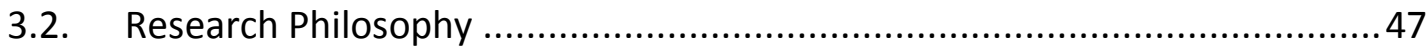

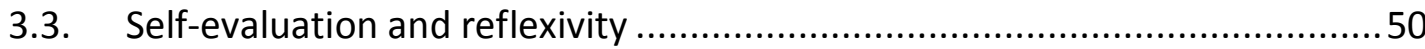

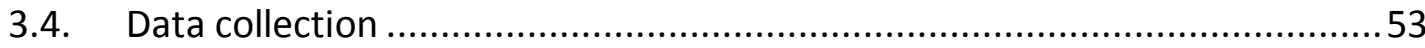

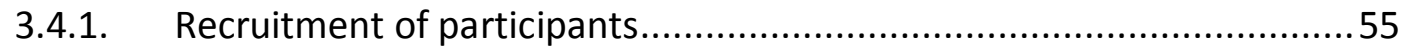

3.4.2. Individual and group interviews with the immigrant-hosts.................60

3.4.3. Individual and group interviews with the VFs and VRs.........................62

3.5. Analytical framework in examining social interactions between immigrant-hosts

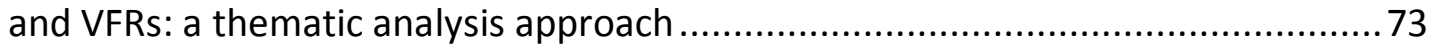

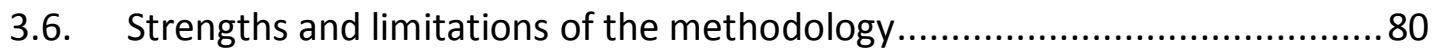

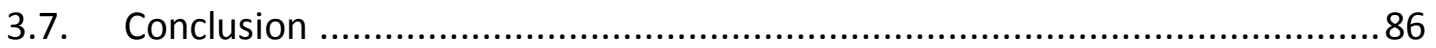

CHAPTER 4 - Analysis of the social interactions between immigrant-hosts and visiting friends 


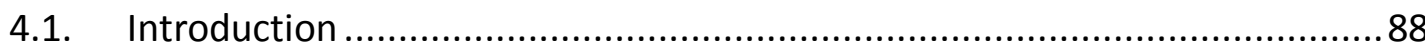

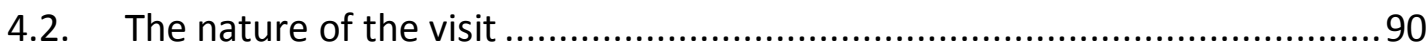

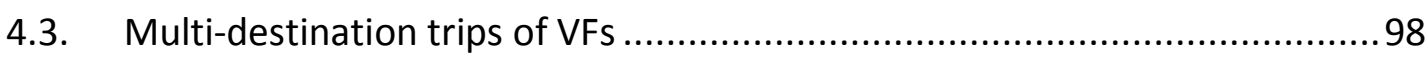

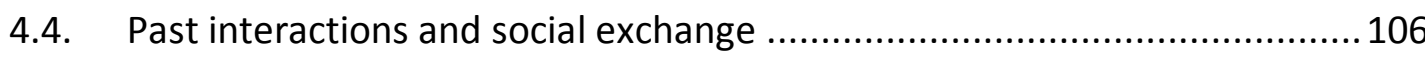

4.5. Emotional solidarity: connectedness in togetherness............................. 112

4.6. Cultural beliefs and behaviours on hospitality ......................................119

4.7. Meanings of the visit among friends................................................. 130

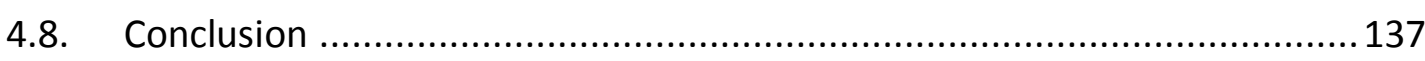

CHAPTER 5 - Analysis of the social interactions between immigrant-hosts and visiting

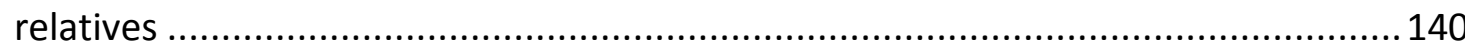

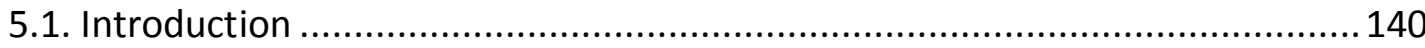

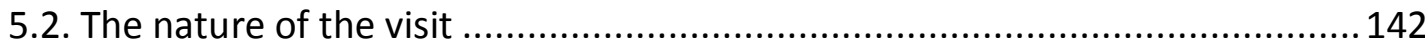

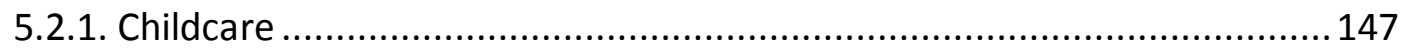

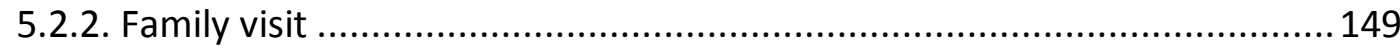

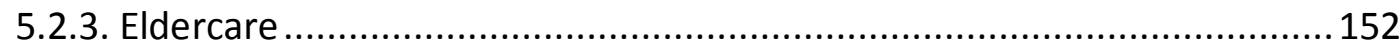

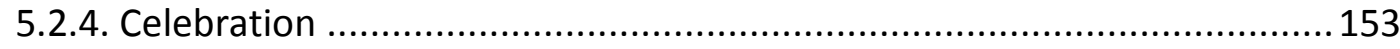

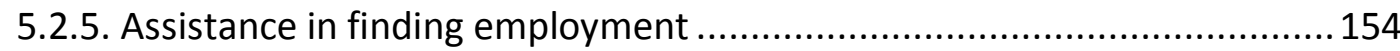

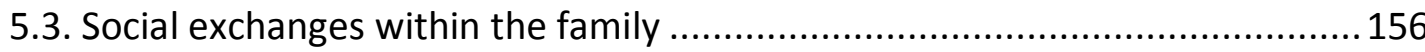

5.3.1. Interdependence of immigrant families and their visiting relatives ......... 156

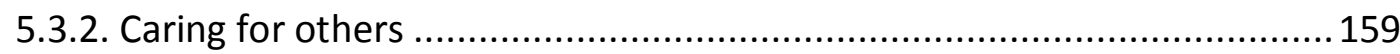

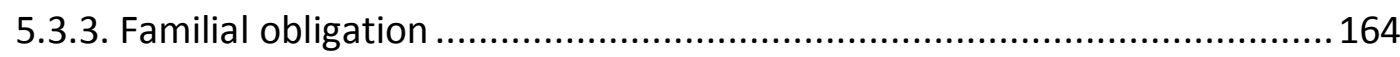

5.4. Familial solidarity: social identities and shared social interactions .................166

5.4.1. Hospitality and sociability between immigrant-hosts and VRs ................167

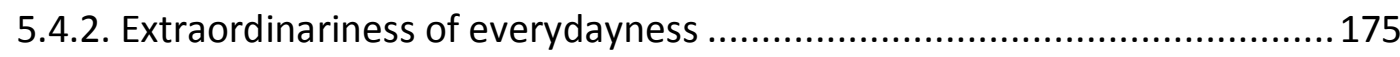

5.5. Cultural identities in New Zealand ........................................................... 181

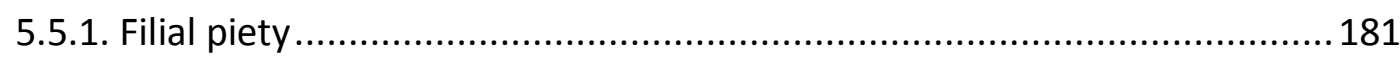

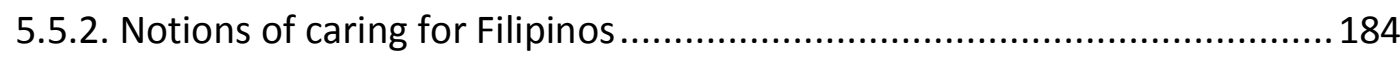

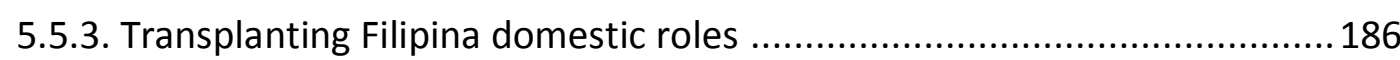

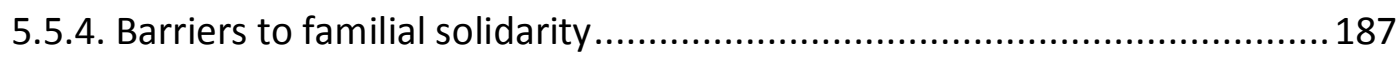

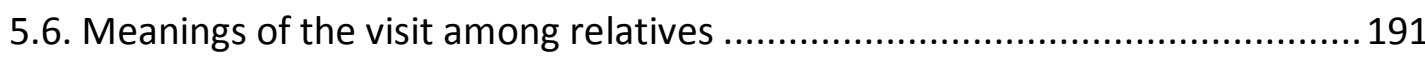

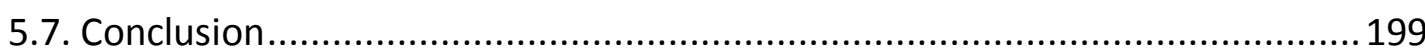

CHAPTER 6 - Qualitative metasynthesis and theoretical interpretation of the social interactions between immigrant-hosts and VFRs....................................................201

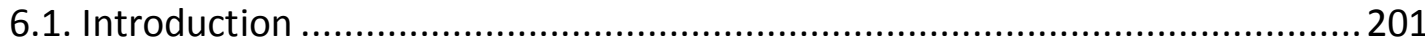

6.2. Reframing the conceptual framework on the social interactions between

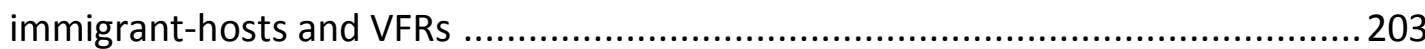

6.3. Nature of social interactions between immigrant-hosts and VFRs .................209 
6.4. Social exchanges between immigrant-hosts and VFRs: trust, reciprocity, and commitment

6.5. Emotional solidarity between immigrant-hosts and VFRs: sharing and giving of oneself

6.6. Cultural influences in the social interactions between immigrant-hosts and VFRs

6.7. Interpretations of social interactions between immigrant-hosts and VFRs .... 228

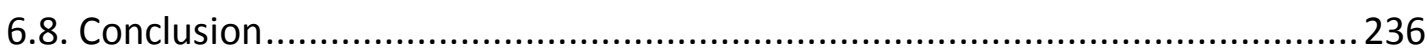

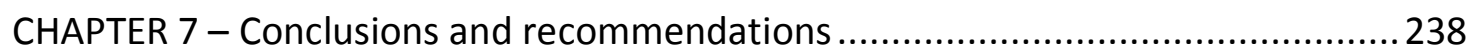

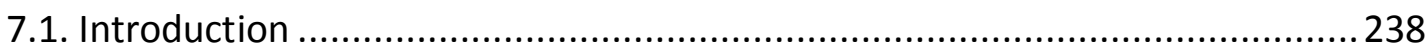

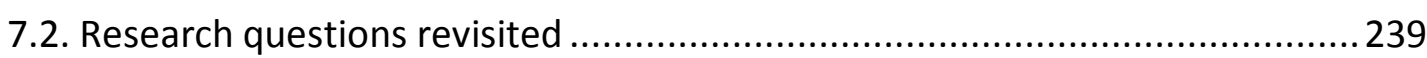

7.3. Theoretical contributions ...................................................................... 246

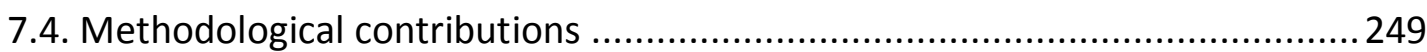

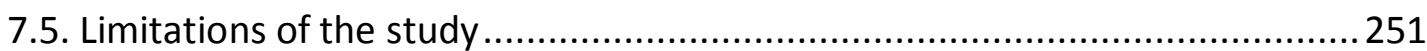

7.6. Implications for the tourism industry and policy.........................................256

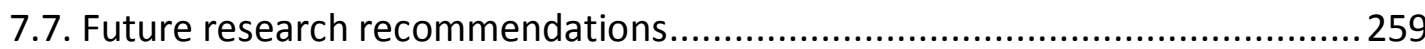

7.8. Conclusion: immigrant-hosts and VFRs - a kaleidoscope of social interactions

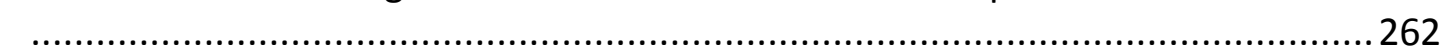

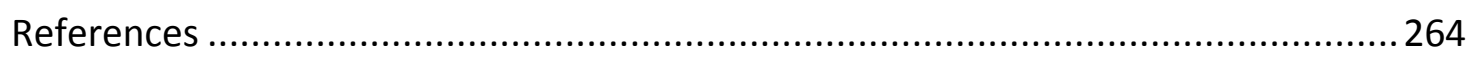

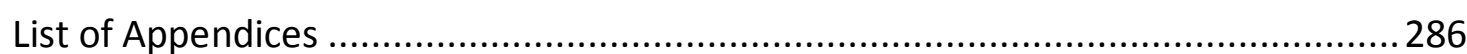

Appendix 1. E-mail permission to the internet community moderator. ...................287

Appendix 2. E-mail announcement to the internet community. .............................28

Appendix 3. Information sheet for the immigrant-host family interviews in New Zealand.

Appendix 4. Immigrant-host family in New Zealand/visiting friends and relatives in the Philippines Consent Form.

Appendix 5. Individual interview guide for the immigrant-host family in New Zealand.

Appendix 6. Group interview guide for the immigrant-host family in New Zealand. 295 Appendix 7. Information sheet for the visiting friends/visiting relatives in the Philippines. 296

Appendix 8. Individual interview guide for the visiting relatives/visiting friends in the Philippines.

Appendix 9. Group interview guide for the visiting friends/visiting relatives in the Philippines. 


\section{List of Figures}

1.1. Share of migrants from developing countries in selected developed countries

2.3. Social interactions between the immigrant-hosts and VFR travellers: a conceptual framework

3.1. Data collection process followed in studying the social interactions between immigrant-hosts and VFRs

3.2. Location of key provinces and key cities within Metro Manila covered during the interviews in the Philippines

3.3. Thematic analysis of the social interactions between immigrant-hosts and VFRs

3.4. Souvenirs bought by visiting relatives during their recent trip to New Zealand

6.2. Illustrative representation of the intergenerational ties between two families in the context of VR travel

6.3. Illustrative representation of the meanings of the social interactions between the immigrant-hosts and VFs

6.4. Illustrative representation of the meanings of the social interactions between the immigrant-hosts and VRs 


\section{List of Tables}

1.1. Residence approvals vis-à-vis Skilled Migrant Category approval in 2010/11 by source countries

2.1. An initial typology of VFR travel

2.2. Some factors affecting the hosting activities of the resident/host

2.3. Hierarchy of inter-relationship in Filipino culture

3.1. Hermeneutic phenomenology: preliminary guidelines for research in tourism studies

3.2. Characteristics of Filipino immigrant-host families vis-à-vis their visiting relatives

3.3. Characteristics of Filipino immigrant-host families vis-à-vis their visiting friends

4.1. Biographical information on the immigrant-host families vis-à-vis their friend's(s') visit.

4.2. Social Interactions between Halcon family and visiting friend

4.3. Social Interactions between Pinatubo family and visiting friend

4.4. Social Interactions between Taal family and visiting friends

4.5. Social Interactions between Ragang Family and visiting friends

5.1. Biographical information on the immigrant-host families vis-à-vis their relative's(s') visit

6.1. Nature of the social interactions between immigrant-hosts and VFs and immigrant- hosts and VRs 


\section{CHAPTER 1 - Introducing the study}

\subsection{Introduction}

In this thesis, I will explore the question: "How are social interactions between immigrant-hosts and their visiting friends and relatives understood and interpreted by them?" This study is consequently focused on the types and variety of social interactions between first-generation immigrants and their visiting friends and relatives (VFRs), in particular first-generation Filipino immigrant households, acting as hosts in New Zealand, and their VFRs from the Philippines, acting as guests. By studying the dynamics of family and friendship relations as expressed from recollections and maintained by exchanges and emotions, the research examines how the host family unit and its individual members interact with their respective visiting friends (VFs) and visiting relatives (VRs), and vice versa, from an individual and group perspective through post-visit interviews. Upon the return of the VFRs to the Philippines, the host families in New Zealand - which I will refer to in this study as "immigrant-host(s)" have been interviewed about their recollections of their experiences in interacting with their respective VFs or VRs. Afterwards, qualitative interviews with their respective guest(s) were conducted in the Philippines about recollections of their experiences in interacting with their immigrant-host family/friend(s) in New Zealand. This introductory chapter presents the background to the study and the researcher, highlighting research gaps and providing context regarding VFR travel, permanent migration, and VFR travel in New Zealand. The overall objective and the research questions guiding this study are presented as well as the contribution of this thesis to wider knowledge. 


\subsection{Background to the study}

My interest in studying VFR travel and immigrants stems from being Filipino with the majority of my relatives living overseas. My own identity is influenced by the fact that immigration has become a major element in Filipino society (see Martin, Abella, \& Midgley, 2004). I remember growing up in Manila in the 1980 s and seeing my late maternal grandmother regularly receiving remittances (money sent home by balikbayan $^{1}$ ) or a balikbayan box (a cardboard box filled with gifts usually sent home by a Filipino overseas worker) from her children who were living in the United States (US). On another corner of our ancestral house, I would see my auntie listening to a cassette tape of recorded greetings sent by her husband from Saudi Arabia and sometime later she would often treat my cousins to a meal at Jollibee ${ }^{2}$ when remittances would arrive. At that time there was very little available computer technology, and internet-aided virtual communication did not exist. My auntie would respond to her husband by recording her reply messages onto a cassette tape and wait for another Filipino going to the Middle East to hand carry the tapes and deliver it to her spouse. I also recall that my family would rent an entire jeepney (improvised mass transit based on jeeps discarded by US Forces) to pick up our relatives along with their many balikbayan boxes upon their arrival from overseas at the Manila International Airport. We would bring their balikbayan boxes to our homes and find them full of consumer goods from the West such as chocolates, shoes, lotions, and toothpaste. Of course, I had no idea at that time that a balikbayan in tourism terms refers to a "visiting relative" (or a "visiting friend", depending on the relationship) who is on a return-trip to their former homeland. Eventually, my mother also immigrated to the US and assumed the traditional role of many Filipino expats by regularly sending money and balikbayan boxes containing goods from discount stores such as Walmart or Costco, not only to our family, but the rest of her siblings in the Philippines.

\footnotetext{
${ }^{1}$ In English, a balikbayan is an overseas Filipino and the term applies to Filipinos who are both abroad indefinitely as citizens and permanent residents of a different country, and to those Filipino citizens abroad for a limited, defined period, such as on a work contract (including those working on a cruise ship) or a student.

2 The Philippines' leading fast-food chain brand. It is a Filipino multi-national chain of fast-food restaurants serving American-style fast-food with Filipino-influenced dishes having several locations in North America, Middle East and Southeast Asia where Filipino migrants live.
} 
Balikbayan boxes presumably represent one of the several types of relationships and exchanges between Filipino immigrants and their families in the Philippines. Several social relationships between and amongst Filipinos in the Philippines and Filipinos overseas have also been examined by other scholars. For example, Aguilar (2009) examined the meanings of houses as an investment in memory that are built through remittances by Filipino migrant labourers and constructed on their behalf by their relatives but are left unoccupied waiting for the return of the balikbayan and in the benefit of kinship and community ties in the present and the future. Meanwhile, McKay (2007) investigated the meanings of remittances as distributed through extended family networks resulting in economic exchanges and emotional intimacy that are deeply entangled. However, in a globalized world where families and friends are widely dispersed, what makes VFR travel different is that family and friends are co-present and have an opportunity to reconnect.

As a result of my experiences and having moved to New Zealand in 2011 to pursue my $\mathrm{PhD}, \mathrm{I}$ became keenly interested in studying VFR travel. My interest and reflection brought me to the realisation that families and friends are a significant portion of the tourism as relationships are vital in ensuring that travel continues between and among places. While VFR travel is not a "sexy" subject (Backer, 2012b), it is undervalued these days (Backer, 2012a; Jackson, 1990) and is still in its nascent stage as an area of academic study (Griffin, 2012) since it is an overlooked segment of tourism. I will examine the meanings and interpretations of the social interactions of Filipino immigrants here in New Zealand with their VFRs from the Philippines. Beyond the production and consumption (supply and demand) of VFR travel, I see the value of social interactions in VFR travel as integral to this study and of eventually disseminating my research to tourism practitioners (Crompton, 2005). Tourism practitioners could potentially benefit from an understanding of the ties between hosts based at a destination and their guests. 


\subsection{Research context}

VFR travel accounts for a significant portion of international and domestic travel. This segment is likely to grow over the next 20 years (Backer, 2012a) as VFR travel is directly proportional to migration as immigrants settle in their receiving country and VFRs extend and develop social relationships with the migrants from their former homeland(s). In addition, VFR travel itself can often lead to the permanent migration of the visiting friend or relative. VFR travel and migration enjoy a distinctive relationship as in most cases migration may create the conditions under which VFR travel occurs and vice versa. Similarly, VFR travel extends and develops pre-emigration social relationships between migrants and those guests who come to visit them in their new homeland. Indeed, globalisation has transformed tourism and migration into interrelated fields.

Being a tourist has various attributes - it requires travelling, visiting, and having a leisure experience (Leiper, 1979). In contrast, Boyne et al. (2002, p. 246) recognise that VFR visits are trips but not typically tourism trips as VFR travel may become more of an obligation on the part of the traveller (e.g., attending funerals, weddings, taking care of an elderly person or new born child). Backer (2012a) provides a definition of VFR travel as a form of travel involving a visit whereby either (or both) the purpose of the trip or the type of accommodation involves VFs or VRs. For consistency, this thes is will therefore use the term "VFR travel" throughout rather than "VFR tourism."

This thesis examines social interactions between first-generation Filipino immigrant households as hosts in New Zealand and their respective VFs and VRs from the Philippines as guests. In this study, first-generation Filipino immigrants are defined as those who were born in the Philippines to parents of Filipino heritage and eventually emigrated from the Philippines to New Zealand. First-generation immigrants, according to the literature, typically have stronger attachments to their former homeland than succeeding generations (Levitt \& Jaworsky, 2007; Mazzucato, 2004). Only VFRs from the Philippines were chosen as participants and they were interviewed 
after returning home from their visit to New Zealand. The rationale was that the firstgeneration immigrant's(s') cultural identity in New Zealand is still evolving while their visitors who reside in the Philippines are still completely rooted in their homeland. Culture is an essential element in understanding the activities of any social group. Although the immigrant-hosts and VFR travellers may have the same (or very similar) ethnic background, these groups have had very different cultural experiences over the course of their lives as the former may have been possibly influenced by New Zealand culture after residing in their new homeland while the guests who are coming from the Philippines have not been similarly influenced. What is distinctive is the current research potential of New Zealand as a setting due to the recent influx of Filipinos as compared with their long historical migration to other developed countries such as the United States (see Bonus, 2000; Espiritu, 2003; Root, 1997) and Canada (see Barber, 2000; Pratt, 2003; Velasco, 2002).

VFR travel research often asks new questions with respect to the nexus between tourism and migration. Globalisation has driven in scale and variety, the types and subtypes of tourism necessitating alternative approaches to research and practice. Migrants prompt an increase in international visitor flows by extending invitations to overseas guests, while conversely, they become tourists in returning to visit friends and relations in their areas of origin (Williams \& Hall, 2000). Consequently, migration creates the potential for the development of VFR travel (Boyne, Carswell, \& Hall, 2002).

The global growth of immigrant communities has become a major source of tourists thereby developing and extending relationships and kinship in their receiving country. For instance, Williams and Hall (2002) grouped tourism-migration relationships in three ways: tourism and labour migration, tourism and consumption migration, and VFR travel. Of these groupings, these authors have identified VFR travel as the most misunderstood, particularly when investigating its relationship with immigration and emigration. However, studies that examine the relationship between tourism and migration have only analysed it based on production and consumption. Like other tourism sectors, VFR travel is primarily market-driven and therefore research about it 
is more focused on quantification (Riley \& Love, 2000; Tribe, 2006) rather than seeking to understand the meanings of VFR travel as a phenomenon. It should be understood that VFR travel is distinct in that it may be more meaningful than the traditional travel paradigm since friendship and kinship ties are fundamental to the relationship (e.g., Hall, 2007).

The immigrant-VFR relationship is crucial to this research where the role of family and friendship networks in VFR travel remain understudied. An innovative approach of this thesis is that it addresses this gap by not only giving a voice to immigrant-host communities, but to their families including adult children as well as their respective VFs or VRs. I address the social interactions between the immigrant-host families in New Zealand and their VFs or VRs from the Philippines and examine what the familial and social relationships mean to them across vast distances and time. In essence, it also provides an understanding of the meanings attached to their social interactions.

\subsection{The research setting - New Zealand}

The total number of international migrants worldwide has increased over the last ten years from an estimated 150 million in 2000 to 214 million persons in 2010 (IOM, 2011). Some developed countries (e.g., Australia, Canada and New Zealand) have preferred highly-skilled workers and attempted to ensure recruitment of suitable immigrants by implementing a point system which takes into account characteristics such as education, occupation, language proficiency, and age (United Nations Development Programme, 2009). Over the last five decades, there was an increasing share of migrants from developing countries who immigrated to selected developed countries (Figure 1.1). Except for the United Kingdom (UK), there were double-digit increases in the share of migrants from developing countries. The UK's large share of immigrants came from developing Commonwealth nations during the 1960s. However, New Zealand experienced a significant increase in migration from developing countries toward the end of the $20^{\text {th }}$ century and the start of the $21^{\text {st }}$ century. During the $1960 \mathrm{~s}$, 
$10 \%$ of immigrants to New Zealand were from developing countries. This percentage increased to 35\% between 1990 and 2004 .

Figure 1.1 Share of migrants from developing countries in selected developed countries (United Nations Development Programme, 2009, p. 32).

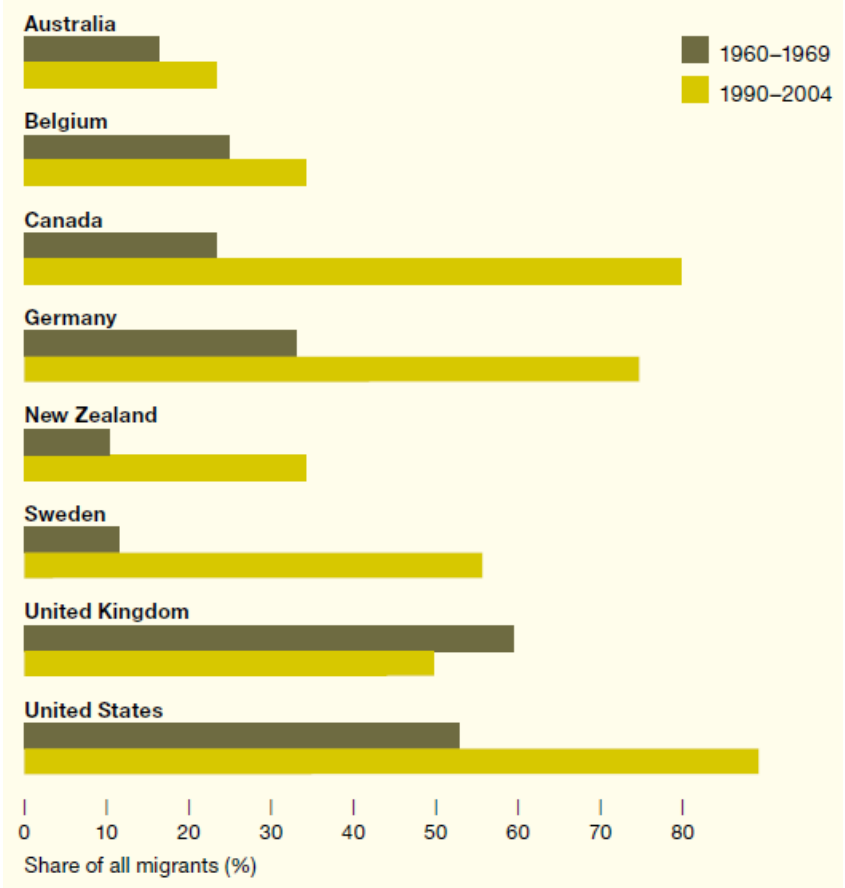

New Zealand is one country that has sought to attract particular types of immigrants and changed its immigration policy in 2003 with the introduction of the "Skilled Migrant" Category which replaced the "General Skills" Category and introduced revisions to criteria for immigrants seeking to reside in New Zealand. Immigration New Zealand has become a more active recruiter of skills and talent and adjusted the "Skilled Migrant" Category to recognise prior work experience gained in a "comparable labour market" which includes the Philippines (Statistics New Zealand, 2007).

The Filipino diaspora is among the largest in the world (Barber, 2000; Pijpers \& Maas, 2013). For instance, a New Zealand Department of Labour report shows that within the annual planning level of 45,000-50,000 places, 40,737 people were approved for permanent residence in New Zealand. Significantly, the Philippines ranked fifth as a top source country for permanent residency approvals and fourth under the Skilled Migrant Category (Department of Labour, 2011) as depicted on Table 1.1. The current population of Filipinos in New Zealand is now estimated to be approximately 35,000 , 
almost $1 \%$ of New Zealand's national population. Filipinos are well represented in industries such as dairy production, health care, information technology, engineering and manufacturing (New Zealand Ministry of Foreign Affairs and Trade, 2011).

Table 1.1. Residence approvals vis-à-vis Skilled Migrant Category approval in 2010/11 by source countries (Department of Labour, 2011).

\begin{tabular}{|l|c|c|c|c|}
\hline Source Country & \multicolumn{2}{|c|}{ Residence approval } & \multicolumn{2}{c|}{ Skilled Migrant Category approval } \\
\hline & $\begin{array}{c}\text { Approved People } \\
\text { (Percentage) }\end{array}$ & Rank & $\begin{array}{c}\text { Approved People } \\
\text { (Percentage) }\end{array}$ & Rank \\
\hline United Kingdom & $6,549(16 \%)$ & 1 & $3,708(17 \%)$ & 1 \\
\hline China & $5,262(13 \%)$ & 2 & $1,723(8 \%)$ & 5 \\
\hline India & $4,218(10 \%)$ & 3 & $2,816(13 \%)$ & 2 \\
\hline South Africa & $3,396(8 \%)$ & 4 & $2,593(12 \%)$ & 3 \\
\hline Philippines & $3,179(8 \%)$ & 5 & $2,478(12 \%)$ & 4 \\
\hline Fiji & $3,037(7 \%)$ & 6 & $1,712(8 \%)$ & 6 \\
\hline Samoa & $1,832(4 \%)$ & 7 & & \\
\hline South Korea & $1,229(3 \%)$ & 8 & & \\
\hline United States & $1,116(3 \%)$ & 9 & & \\
\hline Tonga & $756(2 \%)$ & 10 & & \\
\hline Other & $10,163(25 \%)$ & & $6,182(\mathbf{2 9} \%)$ & \\
\hline Total & $\mathbf{4 0 , 7 3 7 ( \mathbf { 1 0 0 } \% )}$ & & $\mathbf{2 1 , 2 1 2 ~ ( 1 0 0 \% )}$ & \\
\hline
\end{tabular}

VFR is the second largest visitor group in New Zealand after holiday visitors and over the last decade VFR numbers have shown the most dramatic increase, up from 412,000 in 1999 to 743,000 in 2008 (an increase of approximately 80\%). The number of VFR visitors as a proportion of total arrivals has increased from $26 \%$ to $30 \%$ over the same period (Ministry of Economic Development, 2009). However, immigrants in New Zealand have been overlooked as an important human resource for promoting tourism (e.g., Hall \& Duval, 2000). By the end of 2013, the Philippines was New Zealand's $27^{\text {th }}$ largest overseas market with approximately 10,400 international visitor arrivals, increasing from 32 ${ }^{\text {nd }}$ place in 2011 with 8,563 visitors (MBIE, 2013). The significant difference between the numbers of Filipino immigrants settling in New Zealand against the number of Filipino travellers supports the focus of this study. VFRs represent a large potential source of tourism and are an untapped resource in New Zealand's tourism industry that could increase international visitation. Brocx (2003) examines the hosting behaviour of residents in Auckland and also acknowledges the need to utilise the 
resident/host community who have extensive migration links in promoting international visitation to New Zealand.

\subsection{Overall objective and research questions}

This study examines the host-guest interactions of the Filipino immigrant community in New Zealand and their respective VFs or VRs from their former homeland. This complex yet reciprocal relationship will be addressed by examining the immigrant-host and VFR traveller relationship. The overall question that guides this research is:

How are social interactions between immigrant-hosts and their visiting friends and relatives understood and interpreted by them?

Central to the overall research question are supplementary research questions that guide this inquiry:

1. What is the nature of the social interactions between immigrant-hosts and their VFRs?

2. What are the social exchanges between the immigrant-hosts and their VFRs?

3. How do social interactions reflect emotional solidarity between the immigranthosts and VFRs as expressed by their shared beliefs and behaviours?

4. How does culture shape social interactions between the immigrant-hosts and VFRs?

The main purpose of this study is to explore the social experiences and meanings of the visit of the immigrant-host families and their respective VFs or VRs across the temporal dimensions of the pre-visit, the during-visit (actual visit), and post-visit (after the VFs/VRs departed New Zealand). The supplementary research questions build on the conceptual framework which will be fleshed out from the gaps in the literature related to VFR travel (discussed in Chapter 2). Most tourism studies on VFR travel are marketdriven and are not focused on the broader experiential dimensions. The first supplementary research question aims to establish the context of the visit. As VFR travel is multi-faceted - involving the perspectives of the hosts and guests (VFs or VRs) - each social interaction is unique as they put meanings to their experiences. The second and third supplementary research questions provide a conceptual 
understanding of the complexities of social interactions between immigrant-hosts and VFs or VRs using two theories: social exchange theory and the theory of emotional solidarity. These two theories are not always considered in relation to culture which is addressed in the fourth supplementary research question by presenting distinct ways of understanding social interactions between first-generation migrants in New Zealand and their VFs or VRs from the Philippines. While addressing the central research question, this thesis will also compare and contrast the social interactions between the immigrant-hosts and VFs and the immigrant-hosts and VRs when interpreting the social exchanges and emotional solidarity between and among families and friends.

\subsection{Contribution of the research to wider knowledge}

As a non-Western researcher, my academic contribution is to examine VFR travel through a cultural lens by studying first-generation Filipino immigrants in New Zealand and their VFRs. Specifically, the main contributions of this research will be:

(a) to explore host-guest interactions within a VF and VR contexts;

(b) to utilise different theories for interpreting host-guest social interactions in VF and VR settings;

(c) to understand the micro-level interactions amongst friends and family members;

(d) to examine the social interactions through the multiple-perspectives of immigranthosts and their respective VFs or VRs to further the study of VFR travel encounters, including the voices of the secondary members of the hosting/visiting groups;

(e) to examine hosts and guests who share a similar cultural foundation and recognise that this host-guest interaction between or amongst Filipinos is taking place within a non-Filipino cultural setting (New Zealand); and

(f) to understand the range of interactions between hosts and guests across the temporal dimensions of VFR travel.

In practical terms, this research also reflects the need for destination marketing organisations to consider non-traditional markets and "other" cultures when developing and enhancing experiences at tourist destinations. Studying a specific 
immigrant community and their experiences when hosting friends/relatives in their new homeland will delineate how kinship and friendship are related to host-guest interactions. From the perspective of a tourism scholar, studying culture is necessary in order to understand the social interactions of others such as the hosting experience of the first-generation immigrants who may possibly have a different view and perspective from Filipino residents and yet all were born and raised in the Philippines. As New Zealand becomes more culturally diverse through migration, research on other cultures will assist tourism planners in understanding the values and actions of "other residents." A better understanding of the travel and tourism experiences and interactions of immigrants and their VFRs to New Zealand may provide marketers with insights into host-guest dynamics within a VFR context, thus potentially enabling tourism marketers to create better marketing campaigns.

The intended outcome of this research is to offer a deeper understanding of the meanings and interpretations of individual and group experiences brought about by social interaction, thereby enhancing a reader's understanding of the behaviour of Filipinos, whether as a host or as a guest in New Zealand. The recollection of experiences of the immigrant-hosts and VFRs should be thoroughly understood. This study will hopefully reveal insights that prove to be useful for promoting international travel that may have an impact on both immigrants and their respective visitors.

\subsection{Structure of the thesis}

This thesis is composed of seven chapters including this introduction (Chapter One). It follows a "simple" traditional pattern described by Paltridge (2002) which reports on a single study and has a typical macro-structure: introduction, review of the literature, methods, results, discussion, and conclusion. I seek to understand the meanings and interpretations of the host-guest social interactions which are at the heart of this study. Chapter Two reviews the wider tourism and hospitality literature related to VFR travel and its link with international migration. Then, connections between VFR travel research and studies related to host-guest interactions are examined while providing 
an overview of Filipino culture in order to understand the context of the social interactions between the Filipino hosts in New Zealand and the VFRs. A conceptual framework is presented and discussed in conjunction with the research questions. Social exchange theory and the theory of emotional solidarity are used in order to understand the meanings and interpretation of immigrant-host's(s') and VFR's(s') social interactions. Chapter Three outlines the methodological approach and ethical considerations when conducting studies on VFR travel in the context of host-guest interactions, using a qualitative approach. Also, this chapter provides a detailed explanation about the selection of respondents, the data collection process, the challenges and limitations of the study, and the thematic coding and analysis of the data. It expands on the "interpretivist approach" through hermeneutic phenomenology which underpins this research and presents the analytical framework. Chapters Four and Five present the findings that emerged from the data analysis. In particular, Chapter Four provides context for this study, presenting the data analysis and discussion of the social interactions between the immigrant-host families in New Zealand and their VFs from the Philippines, while Chapter Five presents the data analysis and discussion of the immigrant-host families and their VRs. Chapter Six synthesizes the research findings from the two earlier chapters (Chapters Four and Five) through qualitative metasynthesis and delivers a theoretical understanding and interpretation of the immigrant-host's $\left(s^{\prime}\right)$ and VFR's(s') social interactions. This chapter also revisits the main research question and the supplementary research questions and interprets the data by unifying the conceptual foundations used in the study (social exchange theory and the theory of emotional solidarity) within a cultural context, together with the results and analysis of the research. In Chapter Seven, the key findings are summarized while presenting the contributions to knowledge, policy and practice. 


\section{CHAPTER 2 - Literature review and conceptual framework}

\subsection{Introduction}

This chapter positions this thesis in relation to different scholarly literatures - research that addresses VFR travel, the host-guest relationship, friendships, and families - and presents the conceptual framework of the study. It begins with section 2.2 which highlights advances in VFR travel research and its link with international migration as well as current gaps within studies related to VFR travel. Progress related to VFR travel research and its link with international migration is also highlighted as the subject deals mainly with immigrant communities. Section 2.3 connects VFR travel research with studies of host-guest interactions and how these two groups are represented in scholarly literature. Section 2.4 discusses Filipino culture and family ties to provide a better understanding of the relationships existing between immigrant-hosts and VFR while section 2.5 situates friendship as an academic discourse in relation to VFR travel studies.

The four bodies of literature identified earlier provide the conceptual framework guiding this thesis (section 2.6). The development of the conceptual framework begins by defining the nature of social interaction and its current conceptualisation within the tourism and hospitality literature. Social interaction is then expressed within the context of the relationship between immigrant-hosts and VFRs. Finally, the theoretical foundations of the immigrant-host's(s') and VFR's(s') social interactions are outlined using social exchange theory and the theory of emotional solidarity and the fundamental gaps in examining social interactions are discussed.

\subsection{VFR travel research}

Despite scholarly work in VFR travel over the last two decades, the contribution of this type of travel to the tourism industry is underestimated (Backer, 2012a). The early evolution of research related to VFR travel was catalysed by Jackson (1990) who argued that this form of travel was undervalued. In 1995, research on VFR travel was 
emphasised with The Journal of Tourism Studies which dedicated a special issue to the topic. Results from empirical studies in this special issue show that VFR travel was a significant form of travel. However, what is problematic is that VFR travellers may not self-identify as such and may state that they are simply "on holiday." Hence, official data measuring VFR as the focus of the visit will underestimate the actual size of VFR travel (Backer, 2012a).

Studies of VFR travel have provided a better understanding of its contribution to market analysis and segmentation (e.g., Brown, 2010; Morrison, Wood, Pearce \& Moscardo, 2000; Moscardo, Pearce, Morrison, Green, \& O'Leary, 2000), by considering friends and relatives separately in VFR travel research (e.g., Seaton \& Tagg, 1995); through an examination of the use of commercial accommodation by international VFRs (e.g., Lehto, Morrison, \& O'Leary, 2001); and by assessing its economic benefits (e.g., Asiedu, 2008). Pearce and Moscardo (2005) articulate the substantial scale and socio-economic importance of VFR travel in domestic and international settings and as an integral part of the broad definition of tourism. Moreover, Moscardo et al. (2000) provide five defining features of VFR travel (see Table 2.1): sector (as a major motive/trip type or as an activity), scope (international and/or domestic), effort (shortand/or long-haul), accommodation used (accommodated by friends/relatives, commercial accommodation, or a combination), and the focus of the visit.

Williams and Hall (2000) have established the relationship of tourism to other forms of human mobility including migration. The special edition of Tourism Geographies in 2000 was dedicated to exploring the convergence of tourism and migration through examining the relationship in the context of production and consumption. These authors explore further some of the interdependencies in order to understand tourismrelated migration in relation to contemporary human mobility over space and time including: (1) labour migration; (2) entrepreneurial migration; (3) return migration; (4) retirement migration; and (5) second home ownership. In 2002, Williams and Hall (2002) examined VFR travel within the context of the tourism-migration nexus. Through migration, VFR travel creates a spatial arrangement of friendship and kinship 
networks. However, these authors conclude that this is an oversimplification of VFR travel as there are other reasons for visits to family and friends as VFR trips are undertaken for many reasons.

Table 2.1. An initial typology of VFR travel (Moscardo et al., 2000, p. 252).

\begin{tabular}{|c|c|c|c|}
\hline Sector & Scope & Effort & Accommodation used \\
\hline \multirow{8}{*}{$\begin{array}{l}\text { Visiting Friends and } \\
\text { Relatives as: } \\
\text { major motive or trip } \\
\text { type; or }\end{array}$} & \multirow[t]{4}{*}{ Domestic } & \multirow[t]{2}{*}{ Short haul } & $\begin{array}{l}\text { AFR } \\
\text { (accommodated solely with friends } \\
\text { and/or relatives) }\end{array}$ \\
\hline & & & $\begin{array}{l}\text { NAFR } \\
\text { (accommodated at least one night in } \\
\text { commercial property) }\end{array}$ \\
\hline & & \multirow[t]{2}{*}{ Long haul } & AFR \\
\hline & & & NAFR \\
\hline & \multirow[t]{4}{*}{ International } & \multirow[t]{2}{*}{ Short haul } & AFR \\
\hline & & & NAFR \\
\hline & & \multirow[t]{2}{*}{ Long haul } & AFR \\
\hline & & & NAFR \\
\hline
\end{tabular}

Note: VFR - visiting friends and relatives; VF - visiting friends; VR - visiting relatives; VFVR - visiting both friends and relatives.

In a same vein, research on diaspora in tourism terms has been positioned under the umbrella of VFR travel (Causevic \& Lynch, 2009; Coles \& Timothy, 2004; Moscardo et al., 2000; Seaton \& Tagg, 1995). Coles and Timothy (2004) have identified six distinctive patterns of travel and tourism associated with the spaces and places occupied and travelled through diaspora, including: (1) members of diasporic communities who make trips in search of their roots and their routes with the aim of reaffirming and reinforcing their identities; (2) the search for roots and routes gives rise to genealogical, ancestral or family history tourism; (3) residents of the original "homeland" may make trips to diasporic spaces to discover how co-members of the diaspora have adapted to their living conditions in another place (the opposite of the first pattern) that may be considered VFR travel in the consumption of experiences beyond "home"; (4) diasporic destinations as notable attractions and features on "mainstream", non-diasporic tourists' vacation itineraries either deliberately or unintentionally; (5) diasporic scattering in transit spaces; and (6) destinations, resorts, retreats and vacation spaces developed by diasporic communities in their host state as a result of post-arrival colonisation. 
Moreover, Morrison et al. (2000) have described the relationship between tourism and diaspora as a function of ethnic tourism where people visit destinations with an interest in exploring their own ethnic origins. However, tourism and diaspora have been observed via narrow host-guest relationships such as the mapping out of commercial aspects of the interaction (Causevic \& Lynch, 2009). Studies of tourism and diaspora simply become a function of ethnic tourism. However, much of the research that analyses the relationship between tourism and migration and diaspora in tourism addresses macro-level flows of immigrants and VFR travellers but has yet to address the micro-level interactions between hosts and their VFR travellers. Societies and cultures are not fixed entities, nor are hosts the passive receivers of the stimuli to change that the visitors may bring (Williams, 1998).

Seaton (1994) recognises that VFR as a category is ambiguous as visiting friends and relatives can be a primary motivation for a trip, or merely a trip descriptor, meaning simply that friends and relatives provide accommodation, irrespective of whether they are the main reasons for the trip. In the context of the VFR traveller, studying tourist motivation remains difficult (Pearce, 1993; 2005). VFR is often not the sole reason for travel but more often involves a combination of motives that, when pursued at a destination, result in participation in a variety of activities and not only VFR (Moscardo et al., 2000). Nonetheless, if the objective is to (re)establish the bonds of family or friendship, it implies that the social interaction between the host and VFR may either be a social need where one derives a sense of self through companionship held in esteem with the others (Ryan, 2002) or there is a social obligation between actors.

Recently, Backer (2012a, p. 76) provided a definitional model which can be summed up as "a form of travel involving a visit whereby either (or both) the purpose of the trip or the type of accommodation involves visiting friends and/or relatives." This model (Figure 2.1) highlights three distinct VFR types: PVFRs or "pure VFRs" whose purpose of visiting and accommodation used are for and provided by friends and relatives; CVFRs or "commercial VFRs" who travel with the purpose of visiting friends and 
relatives but stay in commercial accommodations; and EVFRs or "exploiting VFRs" have taken a trip that does not purposefully involve visiting friends or relatives but were accommodated by them.

Figure 2.1. VFR definitional model (Backer, 2012a).

\begin{tabular}{|l|l|l|}
\cline { 2 - 3 } \multicolumn{1}{c|}{} & $\begin{array}{l}\text { Accommodation: } \\
\text { Friends \& family }\end{array}$ & $\begin{array}{l}\text { Accommodation: } \\
\text { Commercial }\end{array}$ \\
\hline $\begin{array}{l}\text { Purpose } \\
\text { of Visit: } \\
\text { VFR }\end{array}$ & & \\
\hline $\begin{array}{l}\text { Purpose } \\
\text { of Visit: } \\
\text { Non-VFR }\end{array}$ & & \\
\hline
\end{tabular}

Moreover, Backer (2010b) reviewed various tourism system models and examined them in terms of their suitability for capturing the complexities of the roles and behaviour of VFR travellers from a whole system approach. Among the models that were examined are: the functional tourism system (Gunn, 1988), the chaos model of tourism (McKercher, 1999), the tourism phenomenon model (Goeldner \& Ritchie, 2009), the tourism system model (Mill \& Morrison, 2006), and the whole tourism system (Leiper, 2004). Of all the models, Backer found Leiper's whole tourism system model (Figure 2.2) appropriate for studying the relationships between tourists (the VFR traveller) and the other elements in the system including: generating region, industry, destination region, and transit route. At the heart of the pentagon-shape structure are the hosts who may be influenced by the other elements in the whole tourism system.

However, these tourism models focus on the macro-level interactions of the different elements of VFR travel. Many of the studies related to VFR travel are focused on economic and marketing aspects while the social and community aspects of VFR travel are underexplored (Griffin, 2012, 2013). In the same vein, there is a lack of understanding of the social interactions that provide the voices of both the VFR traveller(s) and the host(s). Furthermore, Griffin (2012) conducted a content analysis 
of articles on VFR travel from 1990 to 2010 that shows that while quantitative studies were fundamental in shaping VFR travel research, there is a need to focus on developing an understanding of that social aspect that sets VFR travel apart from other forms of travel and tourism. Statistics are not able to convey much about human experiences (Carr, 2010) including the hosting and visiting of families and friends. Therefore, the holistic approach of this thesis proposes to examine the micro-level interactions that put both the hosts and guests at the centre of analysis (detailed further in section 2.6) by examining the meanings of their social interactions.

Figure 2.2. Whole-Tourism Model for VFR travel (Backer, 2008, p. 61).

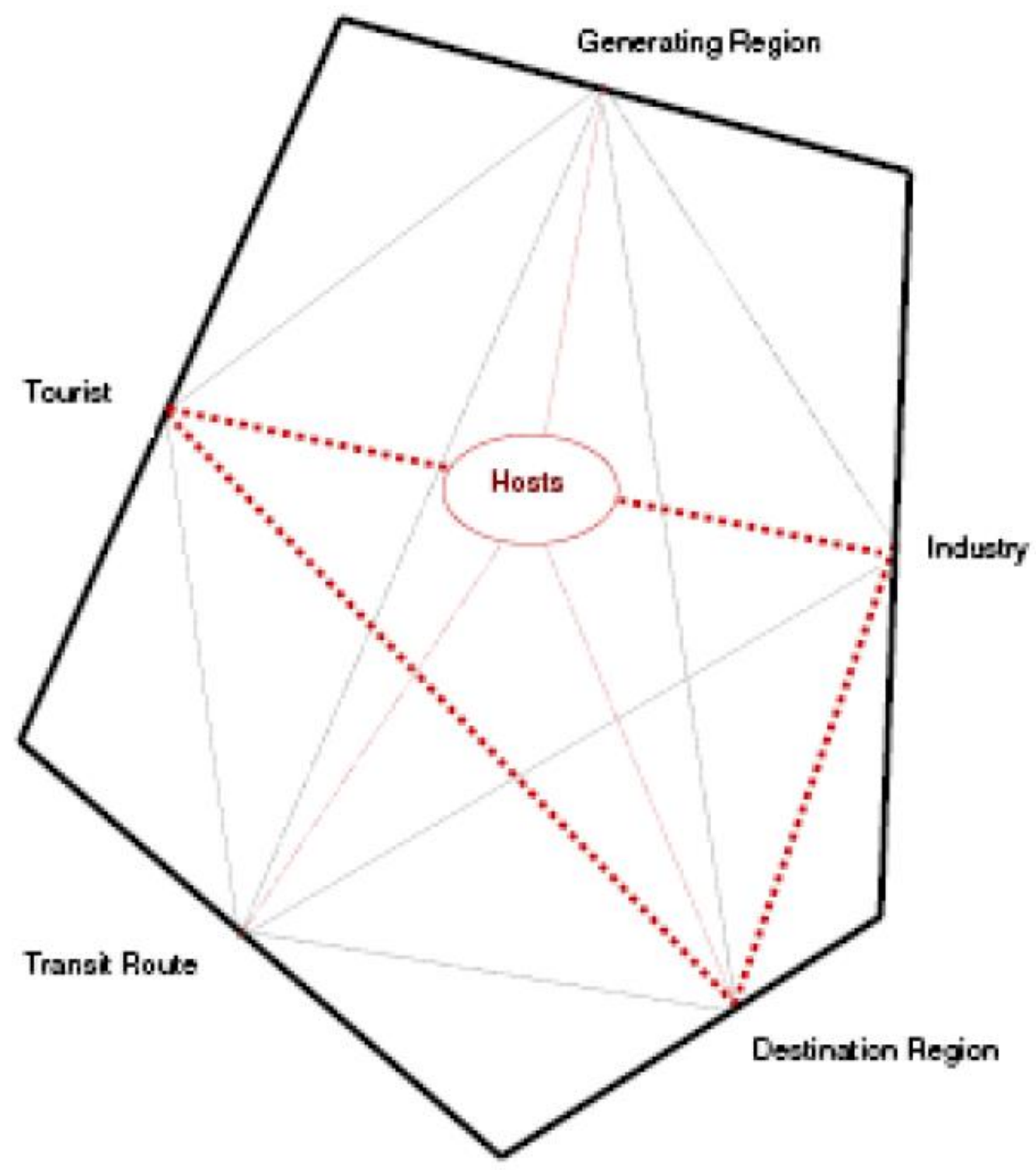


In the context of host-guest relationships, studies of VFR travel have explored the role of the immigrant's friends and relatives as hosts in their country of origin (e.g., Obsequio-Go, 2003; Obsequio-Go \& Duval, 2003). These studies emphasise the role of the Filipino relatives and friends who act as hosts to the immigrant and provide them with travel information. However, there are no empirical studies which provide both a conceptual understanding of the exchanges between immigrant-host families with their respective VFRs when the former expand their roots and assume the role of the host in their new homeland, and how ties of relatedness are still maintained across distance with their relatives or friends. Likewise, the conceptualisation of the meanings of the visit for immigrant-host families and VFRs are not completely understood. Nonetheless, these studies have begun examining how migrants explore their roots as they return to their former home country.

Immigrants become a "pull" factor for their VFRs to travel and visit them in their new homeland. They are an essential element of tourism, particularly for those who travel primarily for VFR purposes (Gheasi, Nijkamp, \& Rietveld, 2011; Hung, Xiao, \& Yang, 2013). However, there is a need to analyse the characteristics and attitudes of immigrant communities. Empirical studies on the role of immigrants as hosts in the VFR travel experience remain scant. Examining social interactions within immigrant communities with their friends and relatives who visit them will give them a voice and provide understanding of the meanings of their hosting experiences. Sheller and Urry (2004) observe that as "hosts" are increasingly from elsewhere, they are no longer static objects of the tourist gaze but are themselves agents in motion. As firstgeneration immigrants are residing in their new homeland, they still can benefit and learn by becoming more familiar with experiences and activities that their "new home" could offer them while performing their hosting role. Therefore, tourism has the potential to transform, contest, and communicate hosts' evolving cultural identity (Lacy \& Douglass, 2002; Steiner \& Reisinger, 2006). There is a need to examine VFR travel beyond the demand side (tourists/guests) but also from the supply side including the characteristics and behaviour of the hosts (Shani \& Uriely, 2011; Young, Corsun, \& Baloglu, 2007). It is also necessary to examine and detail the micro-level interactions 
of the hosts and guests as they are connected to each other (i.e., through kinship or networks) which is discussed in the next section.

\subsection{The host-guest interaction}

The host-guest paradigm was first developed in the seminal collection by Smith (1977) and is considered one of the central tenets of tourism studies (McNaughton, 2006). Consequently, edited books by Smith (1989) and Smith and Brent (2001) describe the host-guest relationship as a socially constructed phenomenon. It is actively produced by both hosts and guests who create their own meanings within the context of social interaction that varies by time, space, and culture. Smith's influential contribution has also established hospitality and the related concepts of hosts and guests as fundamental to understanding the social interactions between tourists and local residents in both commercial and non-commercial settings (Lynch, Molz, Mclntosh, Lugosi, \& Lashley, 2011). However, perceiving the host-guest relationship through a commercial lens narrows the interaction to a relationship between the provider and the consumer of services (Causevic \& Lynch, 2009; Tribe, 2004). While the host-guest interaction represents a human exchange, studies that look at this relationship often focus on tourists and their impact on more traditional or "other" social settings. The host-guest relationship fails to recognise that similarities may exist between the host and guest in terms of socio-cultural backgrounds, i.e., social ties or kinship.

Lynch et al. (2011) acknowledge the narrow focus on host-guest interactions that are commercial exchanges and economic activity in hospitality studies. Such perspectives also prevail when examining the relationship between tourism and diaspora, where diasporas are treated as markets rather than groups with social relationships (Duval, 2003). Meanwhile, Aramberri (2001) rejects the "host-guest" terminology on the basis that it is not relevant in commercial tourism, suggesting that "service providerconsumer" is more appropriate. Sherlock (2001) also proposes the need to revisit the binary opposition between host and guest which can be a blurred distinction due to the complex flow of residents arriving and leaving again. 
Recent studies done by Backer (2010a, 2010b) suggest the need to consider the role of hosts in VFR travel as they contribute their time and resources to the overall experience of their guest. Immigrants, as local residents, not only serve to attract VFR travellers, but can also be the motivators and instigators for visitors' participation in local tourism attractions and activities which frequently reflect the culture, values, and lifestyles of the community (Slater, 2002). Considering that there are now more than 214 million international migrants all over the world (IOM, 2011), such global movement of people should certainly highlight the importance of international migrants as catalysts in promoting VFR travel. As local residents, immigrants in their receiving countries may show VFR travellers around their new homeland and thus engender a sense of belonging to their community.

Despite advances in VFR travel research, most studies still focus on the VFR travellers and generally neglect the significance of the host in the travel experience (Shani \& Uriely, 2011; Young et al., 2007) which is not surprising given that VFR travel is treated merely as a market segment. Brocx (2003) and Slater (2002) both recommend examining New Zealand residents' hosting role in terms of exerting influence on the choices of activities of their VFRs. Results suggest that hosting VFR travellers in New Zealand is a significant activity for its residents. Specifically, Slater (2002) emphasises the contribution of the host in influencing the travel activities and experiences of their VFR guest(s) (Table 2.2).

Both Shani and Uriely (2011) and Young et al. (2007) relate the significant experiences of hosting VFR travellers. The need for scholarly research on other societies and cultures related to non-commercial hosting may have an impact on the hosting experience (involving behavioural styles of hosting) including social class and ethnic background, duration of the experience, strength of the social ties between guests and hosts and the purpose of the guest's visit (Shani \& Uriely, 2011; Young et al., 2007). Aspects related to why some people host more than others were also recommended for study, notably the dimensions of their personality, social identity, community 
involvement, and place attachment (Young et al., 2007). These recommendations have inspired the researcher to investigate the hosting experiences of first-generation Filipino immigrants in New Zealand.

Table 2.2. Some factors affecting the hosting activities of the resident/host (adapted from Slater, 2002).

\begin{tabular}{ll}
\hline Influence of Resident/Host & \multicolumn{1}{c}{\begin{tabular}{c} 
Specific examples of hosting activities \\
\hline Familiarity
\end{tabular}} \\
$\begin{array}{ll}\text { Tourism product awareness; previous travel } \\
\text { experience or utilisation of tourism products; local } \\
\text { knowledge; information used; word of mouth }\end{array}$ \\
\hline Preference & Selection of tourism products for the VFR traveller \\
\hline Ownership & $\begin{array}{l}\text { Sense of belonging; length of residency; sense of } \\
\text { responsibility; sense of pride }\end{array}$ \\
\hline Socialisation & $\begin{array}{l}\text { Socialisation; personal participation in VFR activities; } \\
\text { personal availability; pre-, during-, and post-visit } \\
\text { activities; confidence/trust; concern and care }\end{array}$ \\
\hline Contribution to resources & $\begin{array}{l}\text { Accommodation; transportation; financial } \\
\text { contribution }\end{array}$ \\
\hline
\end{tabular}

This thesis departs from previous studies of hosting for VFR travel by providing a holistic approach in examining the social interactions between immigrant-hosts and their respective VFs or VRs, including the meanings of the interpersonal relationships between and among friends and relatives. Presently, there are no existing studies that examine the multiple voices involved in host-guest interactions across time and distance where both actors are questioned regarding their hosting/visiting. Often, there is a tendency to look only at one dimension of the social interaction involved in VFR travel - whether hosting (e.g., Griffin, 2013; Obsequio-Go, 2003; Schänzel \& Brocx, 2013; Shani \& Uriely, 2011) or visiting (e.g., Duval, 2003; Hung et al., 2013; ObsequioGo \& Duval, 2003; Shani, 2013). VFR travel is multi-faceted where social interactions involves both hosts and guests encompassing both time and space. However, research on VFR travel is usually informed by positivist approaches (Capistrano, 2013; Griffin, 2012). 
The family is an essential component in VFR travel (Schänzel, Yeoman, \& Backer, 2012) where the relationships can occur between members of host families and their respective guests. Accordingly, Schänzel (2010b) suggests examining the perspectives from a whole-family experiential dimension (discussed further in section 2.4) to include gender (male/female; mother/father), generation (grandparents; parents; children), and group/familial dynamics that include the other members of the hosting/visiting family/group. However, the social interactions between immigrant-host families and their VFRs have been overlooked thus, missing the opportunity to examine the social benefits of family time together and the social and cultural development related to hosting and visiting relatives (Griffin, 2013). After discussing host-guest interactions in the context of VFR travel, the succeeding section will provide a perspective on families in relation to VFR travel with a specific focus on the context of Filipino culture and how it influences the dynamics between hosts and VFRs during visits occurring in a different setting that involves the former's new homeland (New Zealand) and their shared culture (Filipino).

\subsection{Socio-cultural studies on families and VFR travel}

This section discusses gaps that exist in the sociological facets of VFR travel and in particular provides cultural aspects to aid in conceptualising families. Specifically, families can be defined in universal, functional, structural, and inclusive ways (Humble, 2010). This may also refer to a social nucleus formed of two or more people linked to each other by matrimony, blood ties, affinities and/or other ties (legal, economic, or religious) (Bolaffi, Bracalenti, Braham, \& Gindro, 2003). However, this definition may no longer be sufficient as it does not include the social and cultural diversity that constitutes a family nowadays such as cohabiting arrangements, domestic partnerships of homosexuals, long distance relationships, families constituted by second marriages (also known as stepfamilies or blended families), and married couples without children either because of the decision to delay or the option not to have any offspring (see Medina, 2001, p. 13) of which the author is aware. In the context of this research, immigrant-host families immigrating to New Zealand are composed of couples (i.e., husband/father and wife/mother) who recognize their respective "family of 
orientation" which was the family in which they were reared in the Philippines. However, the family that a person establishes with a partner may be referred to as their "family of procreation" (Murdock, 1949).

As this thesis is situated in relation to the hosting experience of a specific immigrant community, Shani and Uriely (2011) highlight the need to focus on the sociological aspects of VFR travel including issues of ethnicity and migration as the latter may have a strong association with VFR travel. Both hosting and visiting are social functions based on relationships (Young et al., 2007). From a social science perspective, these arguments highlight the need for an empirical analysis that seeks to determine how culture and setting affect immigrant-host's(s') and VFR's(s') relationships. Sattar, Hannam, and Ali (2013) also suggest investigating the succeeding generations of immigrants in order to comprehend their identities and their obligations to travel. $A$ study that examines the issues raised by these authors would promote a better understanding of the immigrant-host's(s') and VFR's(s') social interactions which may have implications in promoting VFR travel to New Zealand now and in the future.

Although Griffin (2013) and Schänzel, Brocx, and Sadaraka (2014) have analysed the hosting experiences of immigrants, there are no existing studies that examine the multiple perspectives of the immigrant-host families that include the parents and children. Previous studies have suggested eliciting a whole-family perspective within family tourism research that is composed of a family group defined as consisting of at least one child and one adult (e.g., Schänzel, Smith, \& Weaver, 2005; Schänzel et al., 2012) but this approach has not been utilised in the context of VFR travel. A novel approach of this thesis is that the generational dimensions of hosting are explored as the perspectives of the adult immigrant-host children are included in relation to the social interactions that they had with their VFRs. Likewise, examining the noncommercial relationships between hosts and those of their VFRs along with the gender roles and the division of labour within the hosting household using a cultural lens provided a holistic approach in studying hosts' and guests' social interactions. 
Larsen (2008) suggested the need to de-exoticise tourism theory (MacCannell, 1976; Urry, 2002b) and adopt a non-elitist approach to tourism practices without dispensing with the exotic and extraordinary, but to make space within the theory for 'everydayness.' Analogously, this 'everydayness' in the context of hosting friends and family members is called domestic hospitality (Pilardi, 2010; Schänzel et al., 2014). Such domestic hospitality is offered at home which is not only the physical site where one lives. Underpinning domestic hospitality, Russo (2014) described home as 'shared intimacy' between people who have deep ties. An example of shared intimacy is through sharing food as commensality produces bonding. In all cultures, the act of eating the same food together likely brings friends and families closer to one another (Fischler, 2011; Schänzel, 2013).

However, there is little space for thick sociality and relations of domesticity in dominant theorizations of tourism that contrast tourism with the world of the everyday (Obrador, 2012). Domesticity is defined here as "being at home" where "home" is simultaneously a condition of and a consequence of self-giving (Lévinas, 1991; Russo, 2014). Visiting and hosting friends and relatives is by itself a form of sociality (or sociability) which is an interaction between participants that often involves connections rather than an escape from social relations and the multiple obligations of everyday social life (Larsen, 2008; Schänzel et al., 2014).

Within a home, the socialisation of families becomes a "theatre" of multiple relationships between genders and generations (Morris, 1990). In the context of immigrant families, analysing "home" may need a different conceptualisation as migration is invariably a process that dissociates individuals from their family and friendship networks, as well as from other socially significant referents that have strong emotional connotations (Skrbiš, 2008). In the context of immigrants, Gu (2010, p. 691) coined the term emotional transnationalism which describes "the emotions experienced when immigrants and their children search for behavioural guidance and a foundation for moral judgments from the cultural norms of both their previous and new homeland." Metaphorically speaking, immigrants are housed in a transnational 
space where their cultural identification with their former homeland (e.g., the Philippines) is exposed and influenced by the cultural norms of their new homeland (e.g., New Zealand) which may have an implication for how they may handle social relationships with their guests. Nevertheless, VFR travel becomes associated with reunions as a symbolic congregation of families and friends that are widely dispersed. Using these different constructs in VFR travel research to examine "home" from a generational perspective that includes the immigrant-host parents and the adult children will aid in understanding immigrant families involved in VFR travel.

When examining immigrant-host and VFR interaction in New Zealand, Filipino culture, kinship and social ties constitute important dimensions as these factors provide a perspective as to how Filipinos relate to their family and friends. Hofstede (2011) defines culture as the collective programming of the mind that distinguishes the members of one group or category of people from others. It may also refer to all the symbols, meanings, and values shared by members of a group, in contrast to other groups (Ritzer, 2005). Such symbols or meanings may also include norms, customs, and traditions (Reisinger \& Turner, 2003).

This thesis focuses on one cultural group: Filipinos. Aguilar (2009) described that within the Filipino culture, they create a strong discourse on the family and its centrality to individual and social life. Filipino identity and social status are defined by their families that serve as their major source of social, economic, emotional and moral support (Miralao, 1997). In terms of inter-personal relationships, Filipinos identify with "others" as kapwa (fellow being) which is the core value of their personality and at the heart of Filipino values (Pe-Pua \& Protacio-Marcelino, 2000). Unlike the English word "others", kapwa is not used in opposition to the self and does not recognise the self as a separate identity. Rather, kapwa is the unity of self and others and implies a shared identity or inner self. From this arises the sense of fellow being that underlies Filipino social interaction (Enriquez, 1992). Kapwa is the unity of the self and other and is recognition of a shared identity. Enriquez (1992, 1993; cited in Guevara, 2005) explains further: 
A person starts having a kapwa not so much because of a recognition of a status given by him by others but more so because of his awareness of shared identity. The ako (ego) and the iba-sa-akin (others) are one and the same in kapwa psychology: Hindi ako iba sa aking kapwa (I am no different from others). Once ako (I) starts thinking of himself as different from kapwa, the self, in effect, denies the status of kapwa to the other.

Analysing the meanings of immigrant-host and VFR traveller should be understood in the context of Filipino culture. Enriquez (1993), a prominent figure in Filipino psychology, identified different levels of interrelatedness in Filipino language based on two categories: one for the "outsider" and the other for "one of us" (Table 2.3). He further identified kapwa as the superordinate concept embracing both the "outsider" and "one of us" categories. The concept of pakikipagkapwa encompasses all levels in both categories; it is considered to be an important psychological term. It is not a superficial level of interaction, but refers to "humaneness to its highest level" (p. 161). Pakikipagkapwa is the fundamental ethical relation between the self and other (Guevara, 2005).

Table 2.3. Hierarchy of inter-relationship in Filipino culture (adapted from Enriquez, 1993).

\begin{tabular}{|l|l|l|}
\hline Inter-relationship & \multicolumn{1}{|c|}{$\begin{array}{c}\text { Levels } \\
\text { (in Philippine language) }\end{array}$} & \multicolumn{1}{c|}{ English translation } \\
\hline $\begin{array}{l}\text { One of us } \\
\text { (hindi ibang tao) }\end{array}$ & Pakikipagpalagayang-loob & Level of mutual trust \\
\cline { 2 - 3 } & Pakikiisa & $\begin{array}{l}\text { Level of fusion, oneness, and full } \\
\text { of trust }\end{array}$ \\
\hline \multirow{2}{*}{ Outside category } & Pakikitungo & $\begin{array}{l}\text { Transaction/civility with } \\
\text { Level of conforming }\end{array}$ \\
\cline { 2 - 3 } & Pakikibagay & $\begin{array}{l}\text { Level of adjusting; act of getting } \\
\text { along with others }\end{array}$ \\
\cline { 2 - 3 } & Pakikisama &
\end{tabular}


Examining the hierarchy of inter-relationships within Filipino culture assumes that the social interactions of the immigrant-host and VFR traveller reflect mutual trust and occur between individuals who see each other according to Enriquez (1993) as "one of us." The "we" (or "us") mentality or the so-called tayo-tayo lamang mentality among Filipinos help them to carve a deeper sense of Being with Others (cited in Andres, 1981; Babor, 2007):

Sharing is seen in the Filipino way of life, but unfortunately, it is limited to one's in-group to which one has personal relationships. Within one's group, sharing is not merely dictated by pressure, but it is voluntary. There is justice, charity, love to those who are part of one's group but not to all of society.

Concepts related to gratitude and returning favours are tied to Filipino interpersonal relations and can be incorporated into studying host-guest social interactions in the context of VFR travel. Examination of the host-guest relationship between the firstgeneration Filipino immigrants and their respective Filipino relatives/friends reveals values such as hiya and utang na loob were considered which may affect the course of their social interaction. First, the conventional interpretation of hiya which is "shame" or "'the uncomfortable feeling that accompanies awareness of being in a socially unacceptable position, or performing a socially unacceptable action" is inadequate because it does not take into account the importance of understanding the Philippine language. The more appropriate translation of hiya is not "shame" but "sense of propriety" (Pe-Pua \& Protacio-Marcelino, 2000). Second, utang na loob or "debt of gratitude" is defined as the principle of reciprocity incurred when an individual helps another (Andres, 1994). The person helped then feels obligated to repay the debt in the future when the helper himself or herself is in need of aid, or he or she may repay the debt by sending gifts. It is often not clear when a debt has been fully paid, so that the relationship becomes an ongoing one. However, looking more closely within Filipino culture, it actually means gratitude/solidarity. It is not necessarily a burden as the word "debt" connotes, because in the Filipino pattern of interpersonal relations, there is always an opportunity to return a favour. Many Filipinos who are overseas wish to retain strong ties with their homeland, particularly to family and friends who they left behind (Pe-Pua \& Protacio-Marcelino, 2000, p. 55-56). Similarly, Deloso (2007) 
recognises that the Filipino value of utang na loob may be ambivalent in the sense that it can be used positively or negatively. The author then reinterprets utang na loob not as "debt of gratitude" but as "debt of goodwill" arguing that:

Debts of gratitude are, in general, incurred by people who receive help or favours from others. But to say that a person has a debt of gratitude is not merely to say that he should be thankful for the assistance given. The indebtedness concerned is not confined to actual benefits received. In recognising a debt of gratitude, one also recognises the good will manifested by the benefactor in providing assistant or granting a favour.

While social interactions occurring between Filipino immigrant-hosts and their and VFRs from the Philippines are taking place in a different geographical setting (New Zealand), an important understanding about Filipino families is essential. However, the prominence of families and children in tourism contrasts with the blindness of academic research towards relations of domesticity and thick sociality (Obrador, 2012). The Filipino family is characterized as traditionally consanguineal for blood ties are considered to be so very important that even relationships with distant cousins, aunties, and uncles are commonly recognised. The descent system is bilateral which means that the individual at birth is affiliated with both paternal and maternal group of relatives. The larger kin group of the Filipino consists not only of the consanguineal kin or those related by blood, but also the affinal kin or those related by marriage. The rituals of baptism, confirmation, and wedding also expand the kinship structure because the family of the sponsors acquire kin-like relationships with the family of the sponsored (Medina, 2001). Within the broader discourse on family tourism, the hostguest social interactions in the context of VFR travel then becomes an opportunity for individuals to experience their "true selves" which is at its peak and a ritual experience of such existentially authentic relationships (Wang, 1999). This existential authenticity does not refer to the originality of objects and cultures but to a special state of being and a sense of togetherness (Obrador, 2012). The next section will now focus on friendships as a significant topic that may influence the social interactions between the hosts and guests. 


\subsection{Friendships, social interactions, and VF travel research}

Friendship is generally defined by five characteristics - voluntary, personal, equal, mutual, and affective (Rawlins, 1999). A major and unique aspect of friendship is the absence of formal bonds which makes the relationship voluntary (Wiseman, 1986) as compared with institutionalized relationships such as kinship. Previous studies on friendship and its interactions are usually found in social psychology (e.g., Derlega \& Winstead, 1986; Kenrick, Neuberg, \& Cialdini, 2010) and anthropology (e.g., Bell \& Coleman, 1999) and are undertaken using a feminist perspective (e.g., Friedman, 1993; O'Connor, 1992). Other scholarly literatures have examined friendship among adults (e.g., Blieszner \& Adam, 1992) or gays and lesbians (e.g., Adam, 2013; Macdonald, 2007; Nardi \& Sherrod, 1994; Torre, Manalastas, Sese, \& Catanghal, 2005).

However, the meanings of friendship in the context of VF travel research are absent within tourism scholarship considering that tourism revolves around social interactions (e.g., Kinnaird \& Hall, 1996; Moyle, Croy, \& Weiler, 2010; Papathanassis, 2012; Ryley \& Zanni, 2013). There is a need to recognise the social significance and value of friendship ties (Allan, 1989) when analysing the social interactions between the immigrant-host families and the VFs. Furthermore, Stringer and Pearce (1984) recommend harmonising social psychology and tourism studies particularly when examining social interactions because:

[I]t is through interaction in particular situations that one learns about tourism - itself a social institution - how to experience it, relate to it, and even modify it. Tourism phenomena are rarely encountered and interpreted directly and individually, but rather through other people ( $p$. $11)$.

Analysing the social interactions between the immigrant-hosts and VFs are therefore embedded in a larger context that is imbued with meanings. The role of social interaction in the active construction of self draws attention to how they interpret the meanings of their hosting/visiting and their friendships. After discussing friendships in relation to VFR travel, the next section will focus on families with a specific focus on the context of Filipino culture and how it influences the dynamics between hosts and VRs during visits occurring in a different setting that involves the former's new 
homeland (New Zealand) and their shared native culture (Filipino). The next section brings together the various schemes discussed earlier in the literature to develop a structure that examines the social interactions between immigrant-hosts and VFRs as a phenomenon under investigation.

\subsection{Towards a conceptual framework: a focus on Immigrant-VFR traveller relationships}

This section examines social interactions between the immigrant-hosts and VFR travellers which are at the heart of the conceptual framework. The meanings and interpretations attached by hosts and guests to situations are analysed in this research and this thesis gives a voice to these groups and provides an understanding of their experiences resulting from their social interactions. It is also a process through which two or more social actors reciprocally influence one another's actions (Ritzer, 2005), that is, any behaviour that tries to affect or take account of each other's subjective experiences or intentions. This means that the parties to the social interaction should be aware of each other - have each other's self in mind (Rummel, 1976).

There are two levels of social interaction: co-presence and focused interaction. Copresence is defined as the minimal level of social interaction which occurs when two or more individuals signal (through their bodily and facial demeanour, the use of space, or any other means) their awareness of one another's presence and their accessibility to one another should the circumstances arise. On the other hand, focused interaction occurs when people gather together and cooperate to sustain a single focus of attention (Gahagan, 1984; Goffman, 1967; Murphy, 2001). However, in the context of hosts and VFRs, social interaction is not only an encounter, but is deeply rooted in their relationship as either friends or relatives that cut across time and space. In this research, social interaction is defined as encounters between two or more individuals across time and space to which these individuals attach their own subjective meanings and interpretations. 
Current studies on VFR travel focused on typologies of hosts (Young et al., 2007), hosting experiences (Shani \& Uriely, 2011), considerations in studying immigrant-hosts (Griffin, 2013), and religious obligations of immigrants to visit (Sattar et al., 2013). Furthermore, there is still an element of "othering" when examining social interactions within tourism and hospitality research such as those focused on backpackers (e.g., Murphy, 2001), within and among tourists or guests interactions (e.g., Levy, 2010; Papathanassis, 2012; White \& White, 2008), tourists and guides (e.g., Pearce, 1984), and tourists and hosts (e.g., Pizam, Uriely, \& Reichel, 2000). This "othering" is the imaginary construction of different people by external individuals in their encounter with those who are considered exotic and unfamiliar (Hollinshead, 2000). What separates the social interactions between hosts and guests in the context of VFR travel from the traditional host-guest paradigm (earlier discussed in section 2.3) is that it does not involve strangers or "others", but people known to each other. This study examines social interactions between people who are familiar with each other. An innovative approach is therefore necessary when analysing the social interactions between and among friends and family members as they are specifically aware of their relationships.

Consequently, the succeeding sections of the thesis may use the word "significant other(s)" to either refer to families or friends. In the Western context, significant other is colloquially used as a gender-neutral term for a person's partner in an intimate relationship. In contrast, Owens (2007, p. 4320) defines significant others as "those persons who are of sufficient importance in an individual's life to affect the individual's emotions, behaviour, and sense of self." From a sociological perspective, the broader term therefore would include other relations such as family members and friends.

Figure 2.3 depicts a conceptual framework representing the relationships between the immigrant-hosts and the VFRs across the three temporal dimensions of their activities and incorporates both social exchange theory and the theory of emotional solidarity. The multiple social interactions between immigrant-hosts and VFRs are at the core of the framework and the issue of treating VFR travellers in isolation from their hosts is addressed by providing an opportunity to link these groups. In the conceptual 
framework, VFR travellers are divided into two groups: visiting relatives (VRs) and visiting friends (VFs). VRs could potentially interact differently with their hosts as compared to VFs. Examining the VR and VF behaviour and the experiences they have with their "significant" others, in particular with their hosts, in the context of VFR travel research are essential, as travel and tourism have mostly neglected issues of sociality and how much it is concerned with (re)producing social relations (Larsen, Urry, \& Axhausen, 2007). The result is a current lack of conceptual and theoretical understanding of VFR travel and the host-guest relationship. As well, the meanings and interpretations attached to the range of host-guest interactions that exist are poorly understood. While little scholarly attention has been dedicated toward understanding the social interactions of immigrant-hosts and VFRs which take place in a multicultural setting such as New Zealand, studying VFR travel from the cultural and social perspectives from a non-Western background may be meaningful and beneficial because of its unique nature (e.g., Hung et al., 2013; Larsen et al., 2007) as Western ${ }^{3}$ constructs may not be shared by other cultures and therefore requires fundamental understanding of their differences (Watkins \& Gnoth, 2011). For instance, this approach would also be useful in understanding the context of the social interactions among the country's "other" residents which is essential in formulating strategies in relation to the impact of VFR travel on immigrant-receiving countries such as New Zealand.

This thesis positions New Zealand as a "multicultural setting" where various immigrant communities interact. This study acknowledges the historically bicultural nature of New Zealand where the former tends to stress the relationship between the Tangata Whenua (the indigenous Maori people of New Zealand) and Pakeha (New Zealanders of European descent), the two largest population groups (Hill, 2010). New Zealand is also arguably multicultural due to its emerging ethnic diversity (see Statistics New Zealand, 2014a; The New Zealand Herald, 2013). It is beyond the scope of this study to discuss the increasing complexity of biculturalism and multiculturalism in New Zealand

\footnotetext{
${ }^{3}$ In this thesis, Western is defined as a social construct, rather than its geographic location which refers to more affluent capitalist societies, generally with an Anglo-American and predominantly white, middle class cultural focus (see Bulbeck, 1998).
} 
as the basis of this study focuses on a specific immigrant community and its culture. Nevertheless, this study acknowledges social interactions occurring in New Zealand as a "setting." It also recognises the uniqueness of New Zealand culture such as its conceptualisation of family and whanau (extended family) apart from other immigrant communities in relation to Filipino culture.

Figure 2.3. Social interactions between the immigrant-hosts and VFR travellers: a conceptual framework.

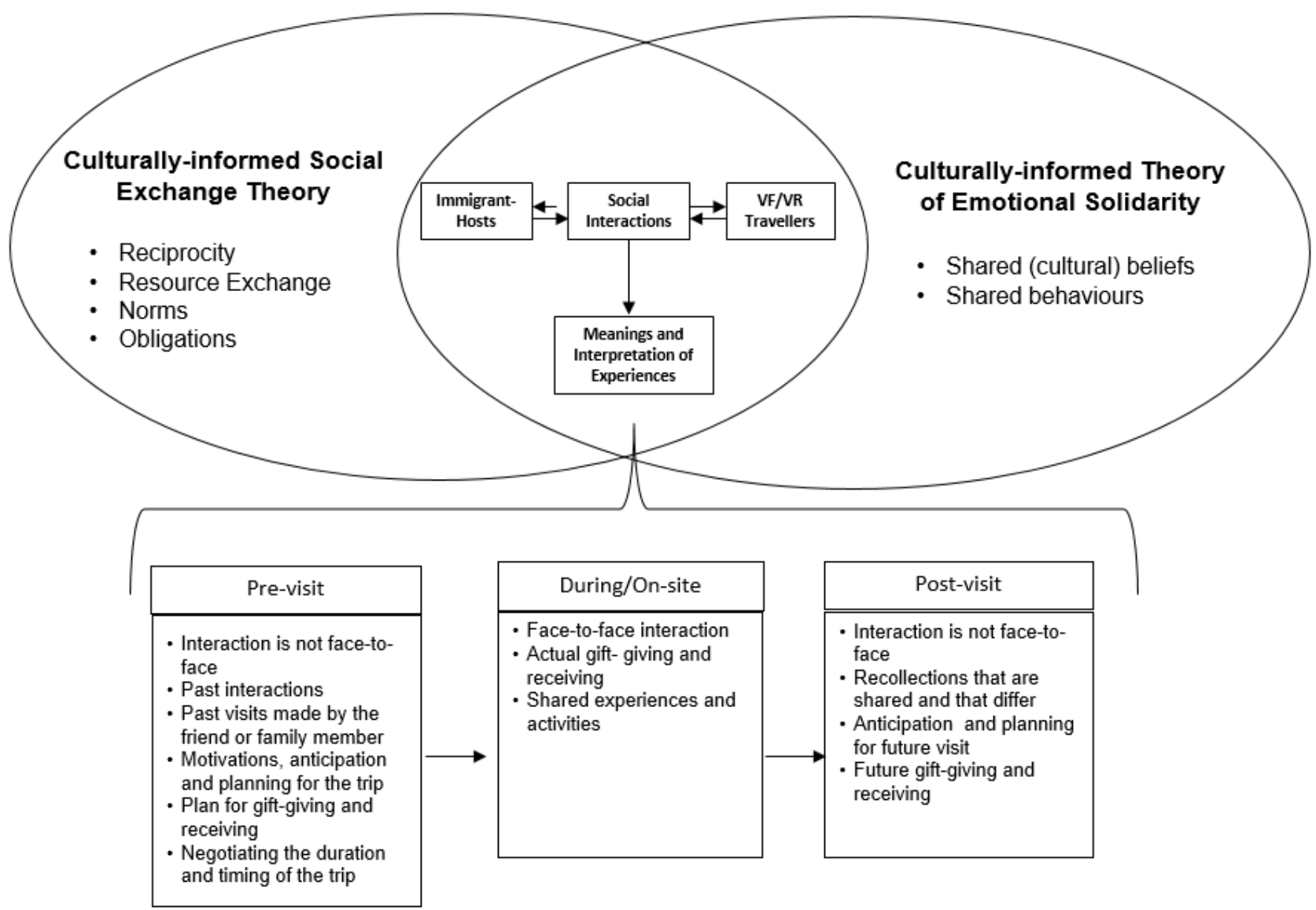


The centre of the conceptual framework depicts the immigrant-host's( $\left.s^{\prime}\right)$ and VFR's(s') social interactions by using social exchange theory and the theory of emotional solidarity in order to identify the attributes of their interactions. Both theories are used in an integrated fashion to guide this research in understanding the significance of hostguest social interactions. These theories have the potential to address relationships between different parties that have some connection with each other - for example, friends and relatives. Likewise, there are concepts that underpin each theory that reflect the relationships between hosts and guests.

Social exchange theory enables investigating relationships at the individual or collective level (Ap, 1992, p. 667) to be examined. Through this theory, the basic unit of analysis is the relationship between actors and is the central object of inquiry; specifically, as this relates to friendship and family ties that connect the immigranthosts to the VFR travellers and vice versa. The theory of emotional solidarity is another dimension that reinforces the interpreting of the immigrant-host's(s') and VFR traveller's(s') social interactions.

The foundational principles of social exchange theory are: reciprocity, resource exchange, norms, and obligations (Cropanzano \& Mitchell, 2005) and, as such, they will guide this study of the social interactions between the immigrant-host and VFR traveller. Reciprocity is regarded as a central tenet of social exchange theory as it is fundamental in the mutual exchange of benefits and also key to the interpersonal transactions occurring between immigrant-hosts and their VFRs. The former's action is contingent on the behaviour of the latter and in terms of resource exchange, the articles of exchange may not only be commodities but gifts that may be tangible or intangible (symbolic). The gesture of giving may also be interpreted as a "selfish act of generosity" as it tends to generate a sense of obligation to the giver on the part of the receiver which leads to reciprocation with the initial benefactor responding by providing something that is desired by the original giver. Trust is also a necessary component of this interaction because of the unspecified nature or implicit terms of exchange (Turner, 2006). 
When considering social exchange theory, the interdependence between the host and guest is influenced by norms which are informal rules that guide social interaction (Dandaneau, 2007). Blau (1964, p. 93; in Cropanzano \& Mitchell, 2005, p. 882) described social exchange as "an association which entails unspecified obligations and favours that may create or entail future reciprocal obligations and the nature of the return gesture cannot be negotiated." Obligations between the host and guest are articulated further by Aramberri (2001, p. 741-742) as the batch of duties for both sides where the host looks after and is concerned for the material well-being of the guest as the latter becomes a temporary member of the host's family. In the context of hospitality, this relationship is essentially a human exchange and has always been characterized by fundamental and elemental features: a concern for pleasure and welfare of the guest, and reciprocity in terms of benefit, wellbeing, and obligation (Santich, 2006).

Social exchange does not specify the exact nature of either actor's obligation(s) nor ensure a return of the favour or resources and the value is often not specified in advance. There is a general expectation of some type of return but it may be based on reciprocity norms rather than an explicit negotiation (Ritzer, 2005). However Backer (2007) recognises that while VFR travel can be primarily obligatory, this cannot be a generalisation as it can be purely leisure based in other cases.

Another interpretation of social exchange is also reflected within the Filipino culture, particularly stated as utang na loob (debt of goodwill). The dimensions of social interaction in terms of obligations (e.g., Heuman, 2005), reciprocity, and norms may vary across cultures. For instance, the notion of "one of us" (hindi ibang tao) within Filipino culture may exist between the immigrant-hosts and VFRs and is contrasted by "others" and these concepts move beyond the commercial view of host-guest interaction. A culturally-informed concept of social exchange will acknowledge that the basis of exchange may be shaped by Filipino understandings and interpretations of gratitude and sense of what is an appropriate exchanging of favours. 
Beyond social exchange theory, Ward and Berno (2011) suggested considering a range of conceptual frameworks arising from social psychology that can be applied to touristhost relations. These authors recognised that the potential of a mutually beneficial partnership between social psychology and tourism studies has not been systematically explored. In this study, a holistic approach of examining the immigrant-hosts and VFRs relationship that incorporates other theories, such as the theory of emotional solidarity will enable consideration of the various dimensions of social interaction resulting from emotional understandings and intimate exchanges. The theory of emotional solidarity is based on Durkheim (1915/1995) who argued that the most basic of religions have two essential features - beliefs and behaviours - which foster solidarity among its members through interaction. Analogously, the emotions that an individual experiences are jointly produced within the host-guest interaction, making relational or group affiliations a prospective source or cause of the emotions. In contrast to the studies carried out by Woosnam and Norman (2009) and Woosnam, Norman, and Ying (2009) which look at the emotional solidarity between residents and tourists, this thesis examines further the interaction of the immigrant-hosts and VFR travellers, which is not only a social relationship but is modified by a shared culture affecting their emotional solidarity as expressed through their networks, social ties, traditions, kinships and friendships. The notion of culturally-informed emotional solidarity depends upon the values of the shared culture of the immigrant-host and VFR traveller. Emotional solidarity, for instance, is likely to be strong amongst those who share a similar cultural background and similarly, emotional experiences are also shaped by culture (Mesquita, 2001; Mesquita \& Frijda, 1992).

Tourism research should also discuss and delineate alternatives to Western-centric research pedagogy and praxis and readjust the privilege of Western world viewpoints and epistemologies to (re)interpret experiences outside Western contexts (Jennings, 2009). This researcher is a Filipino trained in an Anglo-American academic setting intends to fuse Western-centric research practices/frameworks with his indigenous scholastic training and innate understanding of Filipino culture. The identity and language that this researcher shares with Filipino immigrants (at least, with the parents 
and some of the adult children) and their VFRs, provides this researcher the ability to discern the linguistic nuances and cultural cues and thus, expertly detail and analyse their shared experiences.

The immigrant-host's $\left(s^{\prime}\right)$ and VFR traveller's $\left(s^{\prime}\right)$ social interaction is further examined across the three temporal phases of pre-, during-, and post-visit that represents the meanings of experiences also studied by other tourism scholars (e.g., Gyimóthy, 1999; Schänzel, 2010a, 2010b). When reviewing the VFR travel literature, experiences are only captured within the world-view of the tourist or traveller (Brocx, 2003; Slater, 2002). However, the immigrant-host also shares these experiences and is an integral participant in the host-guest interactions. The role of experiences is particularly important specifically due to its capacity to provide shared meanings through shared experiences (Hall, 2007). While meanings may be shared, hosts and guests could also attach different and/or separate meanings to the same interaction. Capturing the potential range of meanings attached to host-guest interactions therefore provides a holistic approach.

The core product of tourism is the beneficial experiences that are gained (Prentice, Witt, \& Hamer, 1998). Within the temporal dimensions of the social interaction between the immigrant-hosts and VFRs are the psychological processes that concern the anticipation of the visit, the actual visit, and the recollection of the visit (Larsen, 2007). Moreover, VFR travel experiences are multi-faceted and dynamic, resulting from the nature of host-guest interactions (presented earlier in Table 2.1). When considering the temporal phases of immigrant-host and VFR social interactions, the during-visit phase would elicit face-to-face interactions while the pre-visit and post-visit phases involve contact over great distances using technology (e.g., e-mail, telephone calls, Skype, and other means of voice over internet protocol, which is known as "VolP" or non-technological means, e.g., letters and postcards). However, interactions within the during-visit phase may also be technologically mediated when the VFRs make excursions or have short travel by themselves and maintain contact with their hosts, possibly through the use of cellular phones or other technical means. Interaction is 
associated with social processes which involves not only face-to-face encounters, but may also arise out of interaction with others who are physically absent or by sharing past interactions with persons who may be deceased; the actors thus stretch away in time and space in terms of their wider implications for analysis (Turner, 2006). Equally, social interaction is not defined by type of physical relation or behaviour, or by physical distance. It is a matter of a mutual subjective orientation towards each other. Thus even when no physical behaviour is involved, there may be social interaction (Rummel, 1976).

Motivations often incite a deeper understanding of what it is that energizes the individual towards particular activities (Gnoth, 1997). A critical approach of the study is that motivations of the immigrant-hosts and VFRs are both examined from an individual and family/group perspective. In this case, VFR travel becomes multi-faceted where the motivations of the hosts and guests may be influenced or provide a "push and pull" factor depending on who catalyses the interaction in anticipation of the visit such that the visit parameters are inevitably negotiated by both parties prior to the during-visit. In addition, Larsen (2007) argued that experiences are influenced by expectancies and events and that they remain or are constructed in the person's memory. Therefore, the temporal dimensions of the social interactions between immigrant-hosts and VFRs may provide different understandings and interpretations.

A distinguishing feature of VFR travel is that the pre-visit experiences are rooted in the nostalgia of the host-guest relationship and past interactions. The host prepares for the upcoming trip of the guest while the latter may be planning to bring a unique gift which could be specific to the host's former homeland for exchange. Therefore, both actors are surely preparing but in different ways for the upcoming trip. The during-visit experiences explore the host's(s') and guest's(s') face-to-face social interactions resulting from the resource exchanges between them and as understood from their individual perspectives. One possible exchange may be the provision of accommodation by the host to the guest, and the guest having appreciation and showing gratitude for this accommodation may in turn offer to purchase food. In 
fostering emotional solidarity, pakikipagkapwa (humaneness) is expressed by the hosts to make their VFRs feel at home as their guest shows pakikisama (level of adjusting) and hiya (gratitude) for the host's hospitality. The duration of the visit depends on its purpose, the type of relationship shared between the host and guest and on the patterns of their reciprocity, obligations, resource exchange, and cultural norms. Various activities may be shared such as when the hosts act as tour guide, or not shared when the hosts and VFRs spend time on their own.

Post-visit experiences are provided by the recollections of experiences and may lead to anticipation of future interaction(s) or conversely diminish the likelihood of subsequent interaction. It may also entail a shared recollection of experiences as reflected by the memorabilia or souvenirs that they keep. There is also scope for recollections to differ as the members of the host family may have various interpretations of their social interactions with their corresponding guests and vice versa. In this instance, further reciprocal exchanges may be expected in anticipating future gift-giving and visits both in New Zealand and the Philippines. Since most studies related to social interactions are focused on "others", the ability to carry out longitudinal studies involving interactions of participants from a distance remains a challenge as compared with the innovative approach of this research as the interactions of the immigrant-hosts and VFRs will be examined after their during-visit interactions.

The conceptual framework departs from previous studies on VFR travel as it focuses on social interactions among friends and family members and emphasises their relationships at the micro-level as hosts and VFRs. While several studies focus on hostguest interaction, this framework recognises the cultural dimensions of their social exchanges and emotional solidarity. Bringing these concepts together forms the social interaction as a process which is a critical link between the individual and society and through which individuals collectively produce and reproduce culture and social arrangements (Ritzer, 2005). 


\subsection{Conclusion}

This chapter introduces studies related to VFR travel, host-guest interaction, friendships, and families as the four bodies of literature that have shaped the formulation of the conceptual framework. First, the scholarly literature on VFR travel was outlined in relation to international migration and the current gaps related to studies of VFR travel. Then, several typologies, models and definitions related to VFR travel were presented. However, studies related to VFR travel are still examined through a positivist approach which fails to capture the meanings that the actors provide to their social interactions that encompasses time and distance.

While the host-guest relationships were discussed, it is essential to look at them from a non-commercial, sociological, and cultural perspective. There is still an element of "othering" when considering host-guest interactions thus missing the opportunity to recognise that similarities may exist between the host and guest in terms of sociocultural backgrounds including friendship and kinship. Previous studies of VFR travel are one-dimensional which means that the research either focuses on the perspectives of the VFRs or those of the hosts. However, a holistic approach is recommended to examine multiple perspectives involved in the host-guest interactions as VFR travel is multi-faceted where social interactions also involve other members of the hosting or visiting family/friend household or social circle. In addition, social interactions between and among friends are usually found in various disciplines such as social psychology and anthropology and are evidently absent in current studies related to VF travel. In the same vein, the prominence of families and children in tourism or VR travel contrasts with the neglect of academic research towards social relations.

Finally, a conceptual framework for understanding the immigrant-hosts and VFRs social interactions is provided using a culturally-informed social exchange theory and a culturally-informed theory of emotional solidarity as the theoretical foundations. The multiple social interactions between immigrant-hosts and VFRs are at the centre of the conceptual framework. This departs from previous studies of VFR travel as it focuses on social interactions among friends and family members and emphasises their 
relationships at the micro-level as hosts and guests. This conceptual framework will be taken forward in the next chapter to benefit and clarify the research paradigm, methodology, analysis and interpretation of results (Pearce, 2012) in order to explore the immigrant-host's(s') and VFR's(s') social interactions. 


\section{CHAPTER 3 - Research paradigm and methodology}

\subsection{Introduction}

This chapter explains the methodological process that was undertaken. It will also aid in understanding the challenges and issues confronted when conducting this study. The five main sections of this chapter are: (1) research philosophy; (2) self-evaluation and reflexivity; (3) data collection; (4) analytical framework; and (5) strengths and limitations of the methodology.

When examining social interactions, addressing the complexity of social interactions between hosts and guests, both methodologically and ethically is important as it deals with friends and relatives who travel to see one another. Within the context of VFR travel, understanding the range of meanings of host-guest social interactions cannot be addressed through a quantitative approach. Quantitative research is seldom able to fully capture the subject's perspective since it has to rely on more remote, inferential empirical methods and materials which are different from qualitative research which puts emphasis on capturing the individual's point of view. Qualitative approaches provide a more comprehensive understanding of VFR travel, including its meanings as compared to quantitative studies (Griffin, 2012). Through qualitative research, one is able to capture what actually takes place and strive to understand phenomena and situations as a whole (Patton, 1982, 2002).

The main subject of this thesis is the social interaction between immigrant-hosts and their VFRs which is examined through a hermeneutic phenomenology approach by attempting to understand the meanings of their experiences. The study may be considered as multi-sited fieldwork as described by Robben (2007, p. 331) who discussed this method used by ethnographers which is designed around "chains", in this case the relationships between the hosts with their guests, and the connections between them. However, this cannot be classified as ethnography since the researcher did not insert himself into host-guest interactions. 
Individual interviews with the hosts and group interviews with the immigrant-host families were conducted in New Zealand (discussed later in section 3.4.2) in order to explore the meanings of their social interactions with their respective VFRs. This allows for each person to individually describe their experience(s) in a private and familiar setting with more time to explain and expand upon their responses. Commencing the interviews with the individual interviews ensured that responses were not influenced by group interviews and allowed each family member to provide individual meanings to the social interactions that are unique to them. As each family member may be connected to their guest(s) either as a visiting friend or as a visiting relative, there are multiple realities as there are as many different realities as there are knowers (Cunningham \& Fitzgerald, 1996).

Group interviews through the voices of multiple family members create a broader perspective of the multidimensional nature of social interactions as individual views of the world are blended to form family or group belief systems and interactions (Beitin, 2008). Group interviews were seen as a means to explore social interactions between and amongst family members, a crucial dimension of this research. Group interviews meant that there was a group dynamic reflected in the data collection. Consequently, the same interview format was followed in the Philippines (individual, then group interviews) amongst the respective VFs and VRs.

As discussed earlier, this thesis utilised qualitative research as framing for the hostguest relationship through a quantitative approach will be limited when exploring the interpretation of social interactions between the immigrant-hosts and their VFRs. However, given the various issues and challenges when studying VFR travel, in particular, the researcher was confronted with what Jamal and Hollinshead (2001, p. 71) describe in such qualitative research scenarios as "messy" circumstances which produced these specific examples encountered in relation to this study:

a. Difficulty in getting a holistic and readily confirmable grasp of the population in question: 
The heterogeneity of many immigrant-host families in New Zealand created challenges where eliciting a whole-family perspective that included parents and adult children made the project difficult. Convening the entire family was sometimes difficult, especially when children are no longer residing with their parents at the time of the interview - either they have moved out or have other commitments. In these cases, capturing the multiple-perspectives within the host family becomes challenging. This may not be the case when dealing with families that have minor children and would presumably be more dependent upon and therefore still living with their parents.

b. Difficulty in capturing (or even inventorying) the whole of a sought behaviour set, and consequently only "single-frame" /"one-time"/"frozen" identities were captured:

Examining social interactions of hosts and guests entails considering two different aspects of a specific social interaction and analysing the beliefs, behaviours, and exchanges of the hosts and their respective guests. While immigrant-host families in New Zealand were initially invited by the researcher to participate, they would in turn need to explain the mechanics of the study to their guest(s) and convince them to participate in the study. Such difficulty in achieving consent of all the participants was specifically experienced when interviewing VFs (as compared with VRs who are more readily and willingly available). Since the focus of the study is on host-guest interactions, failure to interview the other party (the guest/s) would therefore void any interviews that were completed with the respective immigrant-host family.

c. Difficulty in knowing whether one is analysing one distinct population or several sub-set populations:

As both the conceptual and analytical framework provides the basis for examining social interactions as a unit of analysis, this research has four distinct sub-sets of populations: (1) the immigrant-hosts of the VRs; (2) the VRs; (3) the immigrant-hosts of the VFs; and the (4) VFs. While a whole-family perspective was intended, the study also recognised that the relationship between immigrant-host families and their relatives are "interwoven" depending upon the identity of the host in relation to their 
significant other, or vice versa. In this case, the relationship between the actors are complex such that the classification of VRs being examined will depend on their relationship with the host family (whether as grandparent, parent, sibling, cousin, or in-laws). This was less complicated when examining immigrant-hosts and VFs where the relationship between the immigrant-host family and the guest is straightforward and easily defined as friendship although different household members may have different relationships to the VFs which are less readily classified.

d. Difficulty in completing the research interview because the target individual is coterminously engaged in a welter of other pursuits which thereby only yielded "unfinished" or "incomplete" texts:

As VFR travel involves mobility, difficulty was also experienced in interviewing the guests, which is similar to the second challenging circumstance outlined in part (b) above. Rather than scrapping the individual and family interviews conducted in New Zealand and making those efforts void, it required flexibility and patience on the part of the researcher to meet with their families and friends in the Philippines, to the extent that conducting virtual interviews via the internet is another option that was explored (the disadvantage of doing such is explained in section 3.4.3). Hence, the investigator did all that one can do in order to ensure that interviews were not left unfinished or incomplete. Other difficulties in undertaking qualitative research during the data collection process are detailed in sections 3.4.2 and 3.4.3 of this chapter.

Currently, methodological approaches in studying VFR travel in the context of hostguest social interactions are underdeveloped. Nonetheless, this study considers a holistic approach to examine the multiple perspectives of the hosts and guests. While this thesis is linked to studies related to family tourism, which is another understudied area (Carr, 2011; Schänzel, 2010b; Schänzel et al., 2005; Schänzel et al., 2012), capturing both the individual and collective perspectives was challenging. It was difficult to attain a holistic view and capture several perspectives when analysing hostguest interactions, whether from the perspective of immigrant families or of their 
respective $\mathrm{VF}(\mathrm{s})$ or $\mathrm{VR}(\mathrm{s})$ which will be discussed in the data collection section (outlined in section 3.4).

\subsection{Research Philosophy}

The philosophical perspective influenced the choice of research method based on the gaps identified in the literature and in developing further the conceptual framework (presented in Chapter 2) and analysing interviews (presented in section 3.5 of this chapter). There is a noticeable absence of articles examining VFR travel associated with a qualitative approach that reflects the perspectives of both the hosts and guests resulting from their social interactions. While quantitative studies have contributed to a better understanding of VFR travel, they fail to address social interactions occurring between hosts and guests. A qualitative approach will reveal the interactions between hosts and guests in a more in-depth manner and contribute to complement empirical knowledge (see Corbin \& Strauss, 2008). However, the majority of tourism research textbooks do not address or reflect the theoretical paradigms that underpin tourism research (Jennings, 2010, p. 34).

The underpinning paradigm, an accepted view of the way the world works and informing the research methodology (Jennings, 2009) of the study is rooted in interpretive social science. In particular, phenomenology explores how human beings interpret an experience and transform that experience into perceived consciousness, both individually and collectively, through shared meanings. Phenomenology captures and describes how people experience phenomena - how they perceive, describe, feel about, judge, remember, make sense of, and talk about their experiences (Patton, 2002) .

Phenomenology focuses on the knowledge of what people experience and how they interpret the world. It assumes that there is an essence or are essences to shared experiences (Patton, 2002, p. 106). However, Pernecky and Jamal (2010, p. 1063) argue that phenomenological research in tourism appears to have sought the essence of a 
phenomenon while disregarding the particulars of context and interpretation. Hence, this investigation utilised Heidegger's (1996) hermeneutic phenomenology as the underpinning paradigm which is primarily concerned about human existence as beings and seeking to understand the truths derived from the experiences (Table 3.1.). While hermeneutic phenomenology is understudied within tourism research (Pernecky \& Jamal, 2010), this has a potential to provide increased understanding of being-in-theworld or Da-sein of the immigrants-hosts and of their respective VFs or VRs. This paradigm focuses on "the mode of being human" or the "situated meaning of a human in the world." Likewise, this is also reflected on Chesla's $(1995$, p. 66) study underpinning hermeneutic phenomenology as an approach to understanding families:

Being situated means that we already understand who we are; and such understanding is not cognitive but is lived. Understandings of who we are distinctly are transmitted in the everyday habits and practices of those around us. Aspects of this understanding are common to all human beings; other aspects are culturally or regionally specific and even specific to families.

Unlike phenomenology which is interested in attending, perceiving, recalling, and thinking about the world and human beings as primarily knowers, hermeneutic phenomenology views humans as being primarily concerned creatures with an emphasis on their fate in an alien world (Laverty, 2003). Hermeneutic phenomenology offers an approach to study shared or common meanings of families and friendships and enables the study of phenomena that are difficult to address or examine using a science based on positivism or empiricism. Meanings and practices reside and are transmitted within friendships and the interactions of family members.

Therefore, hermeneutic phenomenology offers a philosophical approach to examine the social interactions between first-generation Filipino immigrant families and their VFs or VRs for this study, as they act as hosts and guests, respectively. Hermeneutic phenomenology guides this study in understanding the lived experiences that people have with respect to relationships and social interactions with each other and the meanings that they attach to situations. This study explores the memories of the immigrant-hosts and VFRs who may similarly or differently recall their pre-, during-, 
and post-visit phases. The reflection on lived experiences is by definition always recollective; it is a reflection on experience that is already passed or lived through (Patton, 2002; Van Manen, 1990).

Table 3.1. Hermeneutic phenomenology: preliminary guidelines for research in tourism studies (Pernecky and Jamal, 2010, p. 1067).

\begin{tabular}{|l|l|}
\hline $\begin{array}{l}\text { Reason for } \\
\text { Research }\end{array}$ & $\begin{array}{l}\text { To study lived experience and understand how experiences are } \\
\text { interpreted and understood (the meanings of these experiences to the } \\
\text { participants involved). }\end{array}$ \\
\hline \hline Ontology & $\begin{array}{l}\text { (Being-in-the-World) Realist: The World and Nature can be accessed by } \\
\text { means of our being-in-the-world: we make sense of our being and life- } \\
\text { world (the world we live in) through reflective representation and } \\
\text { analysis. All understanding of our being-in-the-world is perspectival and } \\
\text { shaped by pre-understanding, historicity, culture, practice, background, } \\
\text { and language. There is "realness" to the world and to our experiences; } \\
\text { Da-sein's involvement plays a key role in constructing "truth". }\end{array}$ \\
\hline \hline Epistemology & $\begin{array}{l}\text { Hermeneutic: The main focus is on interpretation, context, and } \\
\text { language; what counts as "truth" is based on interpretation, } \\
\text { co-construction and reflexive participation. Both the researcher and the } \\
\text { participant are self-interpreting beings who live in the "real" world and } \\
\text { hence both play an important role in the process of arriving at } \\
\text { understanding through dialogue and interpretation. Language plays a } \\
\text { key role. }\end{array}$ \\
\hline \hline Methodology & $\begin{array}{l}\text { Interpretive and dialogic: The researcher seeks to interpret and } \\
\text { understand the lived experience; searches for meaning, analyses, } \\
\text { critiques, and negotiates between theory and data, and is guided by } \\
\text { hermeneutic phenomenology. The focus is on the relationship between } \\
\text { self and other, rather than a "subjective" or "objective" stance. }\end{array}$ \\
& $\begin{array}{l}\text { Method: Interviews and participant observation, writing rich } \\
\text { description aimed at understanding and meaning. Co-construction, } \\
\text { reflexivity, and historicity are important guiding principles to this } \\
\text { interpretive task. }\end{array}$ \\
\hline
\end{tabular}

Hermeneutic phenomenology as an approach to interpretive research uses qualitative methods such as in-depth interviews with individuals who have directly experienced the phenomenon of interest; that is, they have "lived experience" as opposed to having had a second-hand retelling of an experience (Patton, 2002). It presumes that the case of qualitative research in the social sciences departs from static and quantitatively measurable knowledge towards a focus on understanding and expressing that aspect 
of being, which is dynamic, experienced and elusive of the positivist researcher (Jamal \& Hollinshead, 2001).

\subsection{Self-evaluation and reflexivity}

Reflexivity is used here as the practice of researchers of being self-aware of their own beliefs, values, and attitudes, and its effect on their interpretation of the data (Payne \& Payne, 2004). It is the process of reflecting critically on the self as a researcher, the "human as instrument" (Guba \& Lincoln, 1981; cited from Lincoln \& Guba, 2000). My subjectivity is situated in being a Filipino, bilingual, middle-class, raised by maternal grandparents and raised by a single-mother, educated in Anglo-American institutions (US, Canada, and New Zealand) and Philippine academic institutions, with various friends and relatives overseas that I have visited during my many overseas trips. My personal "situatedness" and "positionality" in relation to the location (whether in the Philippines or New Zealand) and the research participants (whether immigrant-host families or VFRs) are both a strength and limitation for this study. I did not grow up in New Zealand but have been studying in the country over the last three years, all the while developing an understanding of New Zealand culture, particularly in relation to Filipino immigrant families in New Zealand.

Such self-awareness is essential because I acknowledge my own limitations, influences, power, privilege, and biases just as well as denouncing the power structures that may surround the study (Castagno, 2012). One of my limitations as a qualitative researcher is my hearing disability. Hence, the use of a hearing aid, a digital recording device and my ability in lip reading allowed me to carry out the tasks of conducting qualitative interviews. As research participants were briefed regarding the study, they were also informed regarding my physical limitation and all the respondents were considerate enough to allow the audio-recording of the interviews even if the interview consent form provided them the option of not having their conversation recorded. 
In contrast, the power of the position that I occupy and represent rests in my social status in the Philippines and my current educational background. However, my background as a Filipino social scientist, conservationist, and feminist researcher guided me towards treating respondents as equals within the research experience. Narayan (1993) suggested the need to acknowledge subjectivity imparted from particular and personal locations and admit the limits of one's purview from these positions. Acknowledging my situation and background undermines the notion of objectivity, because from particular locations all understanding transforms as it becomes subjectively based and forged through interactions within fields of power relations.

Being an international student from the Philippines interviewing first-generation Filipino immigrants in New Zealand initially became an advantage in approaching Filipino immigrant-host parents and when engaging their adult children during the study. In the Philippines, however, there was one participant who shared an impression of this researcher during their interview:

Participant: My colleagues want to go to New Zealand but the airfare is expensive. When they saw you, I told them you've been there and also studying. I showed them the document [Information Sheet for VFRs]. They said, "You must be rich."

Interviewer: Definitely not. I am a scholar and my tuition fees are paid.

Participant: Yes, I told them you are a scholar. But they said, "You will still need money."

Interviewer: That's why I'm just taking a tricycle (very inexpensive local public transit utilised for short travel within the Philippines). I don't have much money.

Participant: I told them, "I don't know. Maybe he is rich."

In this case, while culture is shared between the researcher and the Filipino participant (in the Philippines), status is still determined by where one stands in relation to the "other" (Merriam, Johnson-Bailey, Lee, Kee, Ntseane, \& Muhamad, 2001). Upon arriving in the Philippines, this researcher believed there was a benefit in maintaining a "low profile" when interviewing the VFRs due to the researcher's professional 
background and familiarity with participatory action research (Brzeski, Graham, \& Newkirk, 2001; IIRR, 1998) gained through previous interactions with Filipinos in rural communities. The researcher chose to use public transport and to stay in budget hotels whenever possible during the trips in Metro Manila and in the provinces not only for financial reasons but also in an effort to be discreet, respectful, and socially conscious. There are instances when travel requires going to rural areas or passing through slum areas and as a cultural insider, one should try to "fit in" with the community (i.e., follow the local dress standards or by taking the most commonly used means of local public transport) in order to blend in and put people at ease. These measures were utilised so that guests (usually the VRs rather than VFs who are frequently more affluent) would not believe that the researcher was attempting to insinuate that he has an elevated status by behaving as a successful foreign educated balikbayan who is creating an impression of having a high profile. The low key approach also helped to stretch the modest research funding as well. While intentionally modest travel means and dress were initially intended to not draw attention to an unknown visiting scholar, it may have also prompted the participants to share their experiences in a more comfortable and open manner, which may have had an implication for the research outcomes through the quality of the interviews (see section 3.6).

Furthermore, the use of first person "I", instead of the third person is now accepted in tourism journals such as the Annals of Tourism Research provided that the method deployed by the research justifies and explains its use (Tribe \& Xiao, 2011). Since the underpinning paradigm for this study is hermeneutic phenomenology, "I" am an intrinsic part of the interpretation that emerges and that "I" cannot be "bracketed" out of the process. Therefore, the researcher is an active shaper of knowledge (Pernecky \& Jamal, 2010). Utilising this interpretive stance when analysing the interviews is an engaging process where the orientation towards the phenomenon under study, which is the social interactions between hosts and guests, is the matter of central concern. As a researcher, I accept the notion that while research participants share their own views, there are many possible perspectives on a phenomenon which can be metaphorically described as a prism, where one aspect may be transparent while another one is 
hidden. However, the interpretation of the phenomenon is also actively shaped and influenced by one's own understanding or "situatedness" in the world (Laverty, 2003).

\subsection{Data collection}

Figure 3.1 depicts the data collection process undertaken for this thesis. It is divided into three phases: (1) recruitment of participants; (2) individual and family/group interviews with the immigrant-hosts; and (3) individual and family/group interviews with the VFRs. The criteria for selection of first-generation Filipino immigrant families in the study should meet all the following criteria: at least 18 years old; originally from the Philippines; permanent New Zealand residents or those who have acquired New Zealand citizenship; and those who have hosted a Filipino friend or relative whose visit was made within the last three to twelve months. In this thesis, first-generation Filipino immigrants are defined as those who were born in the Philippines and eventually migrated to New Zealand. On the other hand, VFR travellers for this study were Filipino, at least 18 years old and currently residing in the Philippines. It was decided to interview hosts and guests between three to twelve months after the visit had been made because the key focus of the study is to capture recollections of the trip and understand the social interactions that took place. A very recent visit would mean the

post-visit period would be deemed too short or negligible but may be remembered in greater detail. 
Figure 3.1. Data collection process followed in studying the social interactions between immigrant-hosts and VFRs.
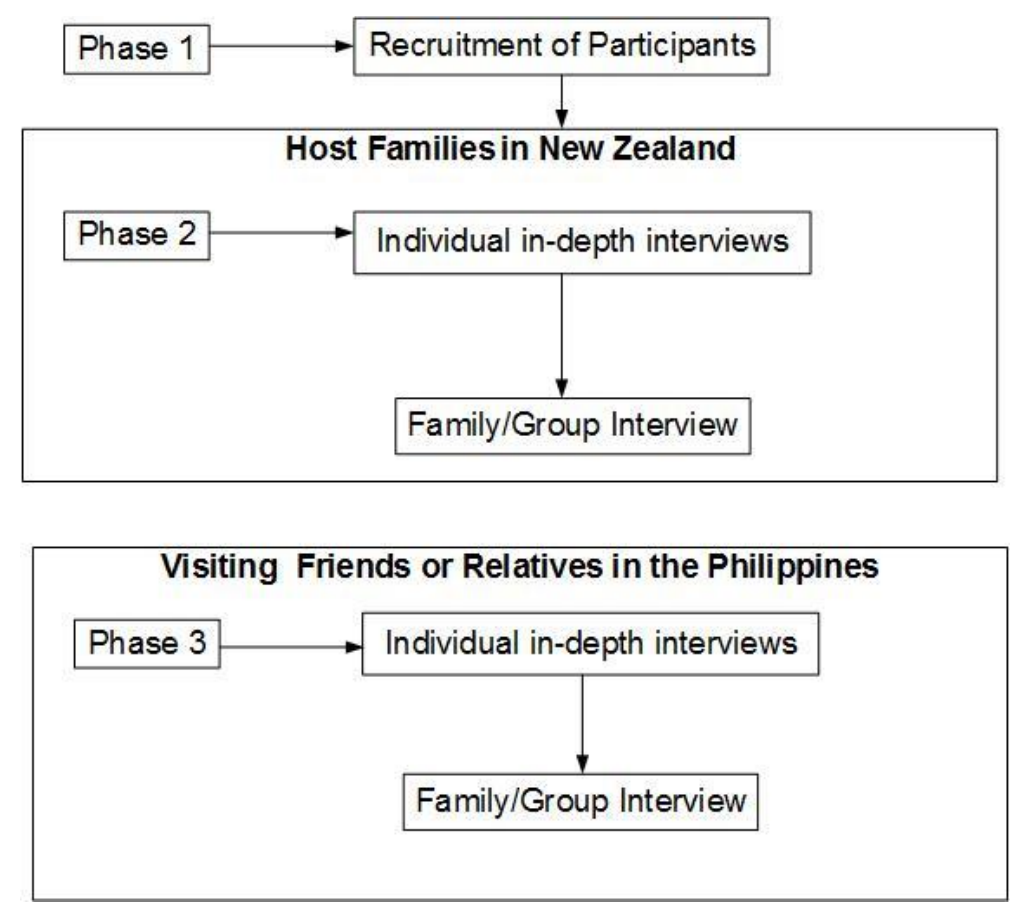

Interviewing the participants is underpinned by a retrospective leisure experience research method that analyses the respondents' recall within one year of recent leisure events and the activities experienced (Yu \& Lee, 2014). The lack of a methodological approach underpinning qualitative interviews for VFR travel when eliciting the perspectives of the hosts and guests has meant that the author had to adopt techniques that are utilised in leisure studies. In particular, the period following the during-visit phase was chosen for academic and practical reasons. In reality, setting the interviews at least three months after the visit was practical since conducting interviews in New Zealand took at least two months. These interviews in the host country were underway while still recruiting additional participants as it was necessary to meet the target number of host families who have hosted for friends or relatives.

Difficulties in recruiting research participants are discussed in section 3.4.1. Equally difficult was arranging interviews in the Philippines where VFRs were geographically scattered throughout the country (see Figure 3.2 in section 3.4.3). Interviewing the guests required travelling long distances to various provinces which was an additional logistical challenge for arranging the actual date of the interview. Therefore, the three 
to twelve month period after the visit for the interview to take place becomes a realistic criteria when examining the social interactions of the hosts and guests across considerable distances.

Due to the apparent limitations and challenges encountered when arranging interviews both in New Zealand and the Philippines, only post-visit interviews with the immigrant-hosts and VFRs were held. The interviewing process for the immigrant-host families in New Zealand and the VFRs in the Philippines began with the individual interviews followed by a group interview. While the benefits of commencing with a group interview would have allowed the participants to become more comfortable within a group setting, the author is a cultural insider which has advantages with regard to commonalities in terms of language, culture, and ethnic background. Due to these similarities, the research participants were able to communicate comfortably with the researcher during both individual and group interviews. Also, individual interviews were expressly completed first in order to determine the individual meanings of the social interactions for the immigrant-hosts and VFRs. In addition, commencing with individual interviews prevented the individual responses from being influenced by other members of the group as well as preventing the possibility that a specific member of the family would dominate the interview process. The details of the data collection process are provided in the succeeding sub-sections.

\subsubsection{Recruitment of participants}

Participants were recruited in various ways: (a) public posting of an announcement/ invitation on the bulletin boards of some Filipino-owned establishments in New Zealand (i.e., coffee shops; restaurants; grocery stores) with verbal permission sought from the owner(s); (b) through internet communities (i.e., mailing lists, newsgroups or websites); and (c) previous acquaintances with immigrant-host families in New Zealand. The use of the internet was more convenient and safe and resulted in a higher response rate when compared to recruiting by using posters. It was also very challenging to convey the message using a poster format as one could not fully indicate all of the necessary information and requirements. 
The announcements used both for the poster and internet communities were written in the English language so as not to favour Tagalog, the structural base of the Philippine language. However, given the diverse ethno-linguistic groups in the Philippines, language differences reflect underlying cultural value differences (Church, Katigbak, \& Castaneda, 1988). As a result, the concept of hosting may be interpreted differently by immigrant communities particularly with regard to their obligations to their guest. Some Filipino immigrants approached during the recruitment phase interpret hosting for VFRs as equivalent to sponsoring their friends and family members to assume residency in New Zealand which includes providing proof of financial support by the host (or sponsor) to bolster the application of their guest, which is one of the requirements of Immigration New Zealand for obtaining a tourist or visitor visa. ${ }^{4}$ Therefore, the Western concept of hosting may be problematic in revealing the meaning of a "host" and as understood from a different language or cultural context.

Difficulties were also experienced in recruiting the appropriate participants who met the study criteria. Some immigrant-hosts who expressed interest in participating in the study informed me after some questioning that their VFRs were visiting at that time (still in the during-visit phase of their social interaction) or their VFRs may have been accommodated in their residence but the primary purpose of the visit was to find a job and their visitor was holding a "skilled-migrant" visa. While Backer's (2012a) definition of VFR travel may be useful, making judgements about which participants to accept in certain cases was necessary to ensure that VFRs from the Philippines who were interviewed were holding a "tourist/visitor" visa as Immigration New Zealand provides a wide range of visas to foreign nationals. For example, there were prospective immigrant-hosts who have accommodated their respective VFRs in their residence and the guests were holding a skilled migrant visa instead of a visitor visa. These families mentioned that they have helped their guests by accommodating them while looking for a job. Categorically, these guests of the immigrant-hosts may fall under EVFRs (exploiting VFRs). However, in this case the legal basis of holding a "visitor visa" was

\footnotetext{
${ }^{4}$ See details of Sponsorship Form for Temporary Entry at http://www.immigration.govt.nz/NR/rdonlyres/31F1BFFC-2BB2-442D-981A30FB01F4C18E/0/INZ1025.pdf which outlines the responsibilities of the sponsor such as financial support, accommodations, and health care.
} 
used for interviewing VFRs while missing the opportunity of examining the social exchanges and emotional solidarity underpinning the relationships between immigrant-hosts and EVFRs.

When recruiting using internet communities, permission was sought from the site moderator(s) of the web-based Filipino associations in Wellington and Auckland before canvassing potential participants from their site (Appendices 1 and 2). There is a need to first obtain permission from the "list owner" (the individual responsible for maintaining the list) or moderator(s) (if any) before posting a request out of courtesy and out of recognition that they would know the make-up of the online community better than this researcher (see Eysenbach \& Till, 2001). The announcement within the internet communities included specific instructions that were given for prospective participants to contact or respond privately should they wish to participate. Some of the internet groups who approved the advertisement were:

a. WlgNew Zealandpinoys@yahoogroups.com - membership consists of Wellington-based Filipino immigrants;

b. AkINew Zealandpinoys@yahoogroups.com - has more than 1,500 Filipino-Kiwi members mostly from Auckland; and

c. WFSA-Badminton@yahoogroups.com - a sports group based in Wellington where most of the members are Filipinos

The aforementioned sites are the groups with which the researcher has built a rapport and had dealings with the moderator(s) and/or the members. Other web-based Filipino associations in New Zealand were also approached but making a connection was unsuccessful due to the moderator wanting to filter information being distributed to their group or because the researcher was considered to be an "outsider" and was therefore denied permission to contact the membership by the site owner(s)/moderator(s). While communicating with another fellow Filipino online (the web-based moderator), the investigator may have been considered to be an outsider because he does not belong to their ethno-linguistic group (i.e., the researcher grew up in Manila and he does not speak regional dialects found in the various provinces of 
the Philippines). On the other hand, some moderator(s) are very strict as to the content and the purpose of the posting(s) on their site(s). The moderator(s) of one group would not grant permission to post a notice to canvass participants as they prefer messages or inquiries by Filipinos that pertain only to immigration and resettlement in New Zealand.

During the proposal stage of the thesis, five Filipino immigrant-host families and their VRs and another five families representing immigrant-host families and their VFs were identified as potential participants for this study. This decision was underpinned by the recommendation of Seaton and Tagg (1995) who suggest considering friends and relatives separately in VFR travel research. However, difficulties were experienced, particularly in recruiting pairs of immigrant-host families and their VFs. When attempting to achieve an equal number of pairs of respondents for host-VFs and hostVRs, the context of each social interaction will vary as hosting or VFR travel is motivated by different reasons. This researcher decided to recruit ten families who have hosted for VRs and should result in enough pairs of immigrant-hosts and VFs to conduct this study but, failed to achieve the goal. Just a few days before finishing the interviews in Wellington and before moving onto Auckland and the Philippines, this researcher was only successful in recruiting only four pairs of immigrant-host families and VFs - then, sometime later, one family who hosted for a VF in Wellington were encountered and they agreed to be interviewed before the investigator's return to New Zealand in March 2013. Therefore, the researcher needed to modify the data collection process necessitated by becoming more flexible and first interviewing the guest (visiting friend) in the Philippines before interviewing the immigrant-host family upon returning to Wellington.

Of the sixteen immigrant-host families enlisted (eleven families hosted for VRs and five hosted for VFs), seven were recruited through the use of the internet. Those recruited through the internet received an e-mail explaining the purpose of the study. Recruiting the immigrant-host families by way of e-mail allowed this researcher to explain the purpose of the study. Some respondents requested an initial telephone call as they 
must have preferred verbal communication for further clarification about the study (particularly the participants who were based in Auckland). However, eight participating immigrant-host families were previous acquaintances of this researcher who he met in New Zealand during several social occasions (i.e., Philippine Independence Day celebration, church gatherings, or birthdays). The last immigranthost family was successfully recruited when the researcher happened to meet them on the bus (at that time there was uncertainty whether the target number of respondents for the immigrant-host and visiting friend pair will be achieved) and through happenstance and persistence in approaching Filipinos at every opportunity (which was earlier explained in relation to difficulties in recruiting host families for VFs in New Zealand).

Establishing a good rapport with the families before formally beginning the data collection was necessary to build trust with the participants. Upon approaching the families, they were told about the mechanics of the research as time was a crucial aspect of the research (such as the schedule of the visit between the immigrant-host family and their respective VFs or VRs who resided in the Philippines; the visit must have been completed and the VFs or VRs must have returned to their home in the Philippines when the interviews took place). While recruiting participants, planning the trip from New Zealand to the Philippines was made contemporaneously and the many domestic trips within the latter travel phase were planned as the logistics of travel were complicated.

Planning the sequence and layout of the interviews was crucial in order to conserve both travel expenses and time. Upon approaching families in New Zealand, they were familiarized with some of the chronological issues related to the project. Host families had to have hosted either visiting relatives or friends between three and twelve months prior to the interviews. Obviously, the visit must have therefore been completed by the time of the interview and the VFs or VRs must have then also returned home by the time the interview took place. 
The immigrant-host families who responded and expressed their interest in participating in the study were emailed a copy of the information sheet including the objectives of the study (Appendix 3) and they were scheduled for individual and group/family interviews. Interviewing the participants in their residence was preferred so as to make them feel more comfortable and more able to focus (Riley, 2012). However, the respondents were given the option of conducting the interview outside of their residence in case they had a preference. For instance, one interview with an immigrant-host female participant who is categorised as "a single-person household" was arranged in a nearby restaurant so that she would not feel awkward or uncomfortable by allowing a lone male to enter her residence.

\subsubsection{Individual and group interviews with the immigrant-hosts}

The individual and group interviews with the immigrant-host families were conducted in phases. The initial phase took place between the first week of September and the first week of October 2012 in Wellington followed by a subsequent phase of interviews in Auckland from the second week October to the first week November 2012. All the interviews with the immigrant-host families were conducted in New Zealand while the guests were interviewed in the Philippines after they had returned home. There were

some similarities between this study and a multi-sited fieldwork done by ethnographers as Filipino families in New Zealand who were interviewed in their residence where most of their interactions may have occurred with their respective friends and relatives prior to the conduct of interviews. However, ethnography requires observation and/or interaction with the participants that usually covers a longer period of time (Lowery, 2001; Robben \& Sluka, 2007). In actively listening to the recollection of experiences by the immigrant-hosts family members in their respective residences, the stories concerning places and circumstances were imagined to be real where the immigrant-host(s) interacts with their guest(s).

The typical individual interview consisted of a one-on-one interview with each adult member of the immigrant-host family (parents and their children who were over 18 
years of age) in New Zealand which was followed by a group interview with the entire immigrant-host family unit. Therefore, each adult family member was interviewed twice (first, individually, then as part of a group). Prior to the interview, the research agreement was first discussed with emphasis on the human ethics procedures/consent form for the immigrant-host families (Appendix 4). A semi-structured interview schedule was used in conducting the individual and group interviews (Appendix 5 and 6). Probing questions were asked throughout the interview in order to elicit more detailed responses. The interview questions were translated into Tagalog while still encouraging the participants to speak in their language of preference, whether it was Tagalog or English. Bilingual Filipinos (including this researcher) typically switch back and forth or intersperse another language concept into conversations (usually English ${ }^{5}$ ), presumably in part because each language provides more precise or easier expression of particular meanings (Church et al., 1988, p. 190). In some instances, the entire interview was conducted in English, particularly with immigrant-host parents who are not Tagalog speakers (e.g., those who came from other ethno-linguistic regions in the Philippines) and in cases where immigrant-host children who were raised in New Zealand and whose first language is English.

The next phase of interviews with immigrant-host families resumed in Auckland from the first week October to the first week of November 2012. A second study city was targeted not only with the goal of achieving the targeted number of respondents for the host-VRs or host-VFs pairs, but to also compensate for the inherent intimacy among Filipino immigrants in Wellington and thus making the research participants less identifiable if they were recruited only in one city/region. In some cases, certain immigrant-host families that were interviewed offered to refer the investigator to other Filipino families in Wellington, but such kind offer(s) of assistance in recruiting were declined so as to ensure other participants could not easily identify each other. Difficulties were also experienced in recruiting a sufficient number of participants in the small and compact city of Wellington which necessitated searching for more

\footnotetext{
${ }^{5}$ English is the official second language of the Philippines and is a medium of instruction and communication within academic institutions and businesses. In certain cases, the researcher or the participant(s) speak in Taglish, an example of code-switching between Tagalog and English.
} 
participants in Auckland where the majority of immigrant families have settled in New Zealand.

The choice to conduct the individual interviews prior to group interviews is justified earlier in section 3.1 as it prevented individual responses from being influenced by group interviews and allowed each family member to provide their individual meanings to the social interactions that are unique to them and in retrospect this researcher still stands by that decision. There would also be advantages to commencing with the group interviews as an initial interview with the entire family group may make interviewees feel more comfortable with the researcher in subsequent individual interviews. While the researcher recognises that conducting an initial group interview could have benefits, in practical terms, it was necessary to conduct the individual interview first as each member of the family/group often arrived home at different times. Since most of the interviews were held in the evening, the researcher had to maximise the time by interviewing those who were immediately available (usually the immigrant-host mother) then moving onto the group interview after the entire family unit had arrived home for the evening. The same format was followed in interviewing groups of VFs and VRs in the Philippines which is discussed in the next section.

\subsubsection{Individual and group interviews with the VFs and VRs}

From the second week of November 2012 to the third week January 2013 (excluding the Christmas break), appointments were made with the respective VRs and VFs of the immigrant-host families in the Philippines. Whenever possible, an advance copy of the information sheet for visiting relatives/friends was provided via e-mail (Appendix 7) similar to those received by their respective immigrant-hosts except for those VRs who are not adept in using e-mail, such as many of the visiting grandparents. A copy of the information sheet was provided to the principal contact person within the immigranthost family of the VRs in the Philippines. Alternatively, a "soft-copy" of the information sheet was e-mailed or coordination with their immediate family members upon this researcher's arrival in the Philippines. It was easier to explain the objectives of the 
study in person and this researcher detected no feelings of hesitation on the part of VFRs to listen as they were welcoming and seemed willing to give their precious time. It was also assumed that the immigrant-host families in New Zealand had oriented or discussed their research experiences with their VFRs and provided them with their anecdotal information with regard to the background of the study and the possible questions they may expect during the interview. The researcher felt the need or obligation to stay longer and visit with the participants in their residence as an expression of pakikisama ("getting along with") as they usually had prepared a meal expressly to be consumed with the researcher during the interview visit. During the course of the visit, most of the time a family member would also ask questions about the investigator's interest in studying VFR travel. The aforementioned technical issues experienced by the VRs were not encountered in distributing the information sheet to the VFs as they all had access to the internet.

The fieldwork in the Philippines was physically demanding as it covered considerable distances (Figure 3.2). Time management was also necessary especially when travelling from the researcher's residence in Manila to the domestic airport on several occasions (even if the distance is only 25 kilometres it can take an hour or more one way in heavy traffic). Travelling for the interviews of the VFs and VRs covered approximately 11,500 kilometres ${ }^{6}$ including various parts of Metro Manila (Caloocan, Makati, and Marikina) and to key places in the Philippines such as Baguio, Bataan and Tuguegarao in the North; Naga City in the Bicol region; Bacolod City in Central Visayas; and Davao City and Cagayan de Oro City in Mindanao. The majority of the interviews with the VFs and VRs were done face-to-face, except for one interview with a VR that was conducted virtually through Skype as the guest had travelled overseas by the time that the researcher returned to Manila. During the virtual interview, intermittent connection issues were experienced and many repeat calls were made to complete the interview and these difficulties were probably caused by limited bandwidth connection. Despite the difficulties, the participant was very cooperative and was willing to answer the questions as both parties agreed to use Skype to facilitate the interview process. While

\footnotetext{
${ }^{6}$ Distance was computed using Google Maps from Quezon City to other places in Metro Manila or the provinces.
} 
the internet has an advantage in facilitating contact between people across distance, it falls far short of communicating what may have been better conveyed in person (such as emotions, visual and aural cues) (Evans, Elford, \& Wiggins, 2008).

A similar or parallel interview process was followed with the VFRs and began with explaining the human ethics procedures/consent form (Appendix 4) for the VFs and VRs, followed by conducting individual interviews (Appendix 8 ) with the respective visiting family member(s) or friend(s) and a subsequent group interview (Appendix 9), if there were multiple visitors. Each visiting family member/friend was interviewed twice (first, individually, then as part of a group). All of the VRs were interviewed in their home, but all of the interviews with the VFs were conducted either in their workplace or restaurant at their request to accommodate their schedules.

Language was not an issue except for one grandparent who suggested that the consent form he signed should have been translated into Tagalog which was unfortunately not possible due to short notice. He was one of the last remaining interviews that were conducted in the Philippines and this issue had not been encountered in previous interview process. Nonetheless, the participant's language concern was addressed by verbally translating into Tagalog each point outlined in the consent form prior to his signing. 
Figure 3.2. Location of key provinces (outset) and key cities within Metro Manila (inset) covered during the interviews in the Philippines (Sontillanosa, 2013).

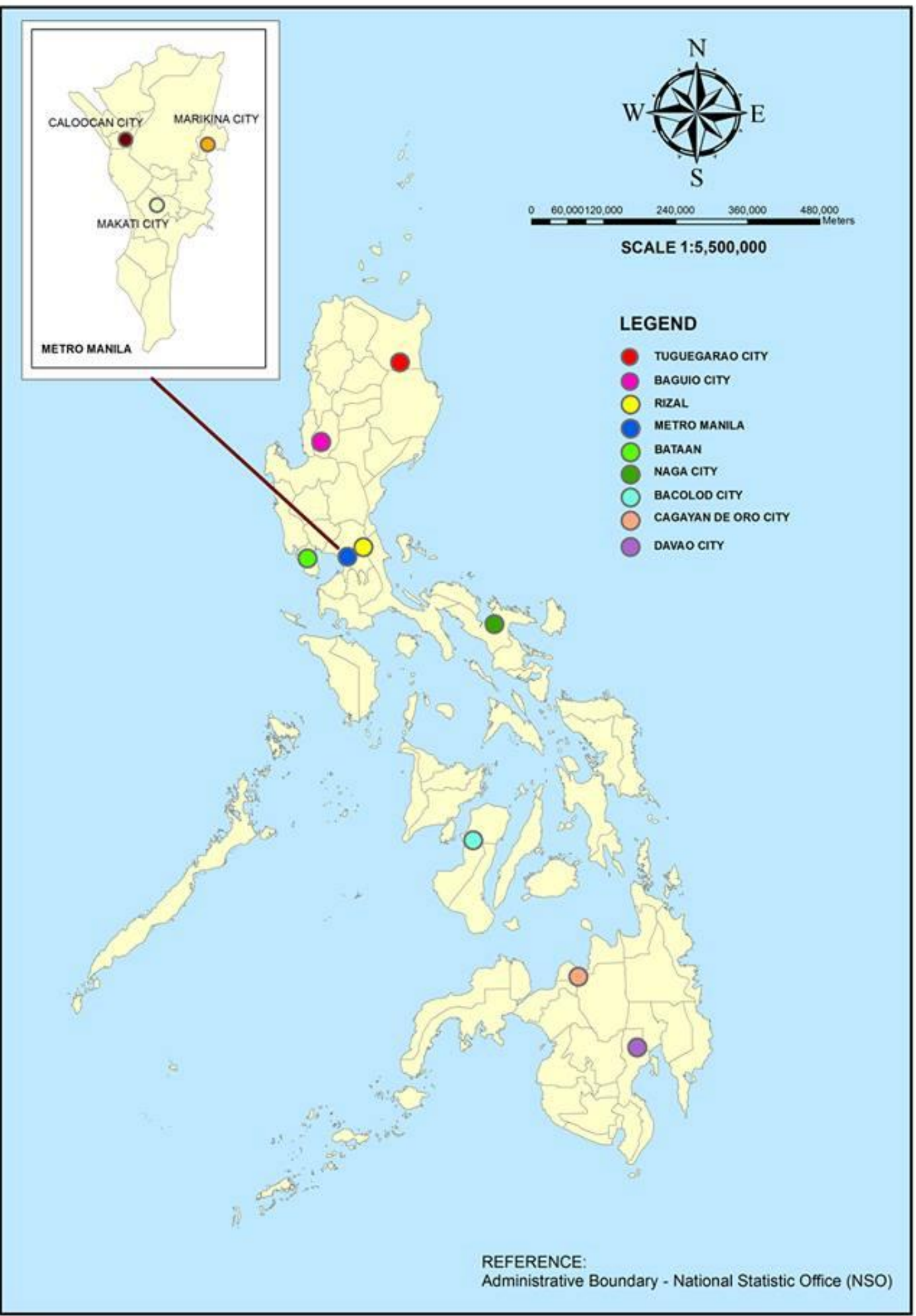


Tables 3.2 and 3.3 show the general characteristics of the research participants using different code names for each host family so, that they can be easily differentiated while still maintaining their anonymity. The pseudonyms selected to represent the immigrant families who have hosted for their relatives and friends are derived from the important biodiversity areas (IBAs) and active volcanoes in the Philippines, respectively. Data collection consisted of interviewing eleven immigrant-host families who have hosted for VRs (28 people, composed of eleven host-mother(s)/wives, ten host-fathers, five host-sons, and two host-daughters) and five immigrant-host families who have hosted for VFs (nine people, composed of one host-mother, two host-wives, one host-father, one host-husband, two members of a lesbian couple, one singlefemale household; and one host-son). Additionally, 17 visiting relatives were interviewed (consisting of eight visiting mothers of host-mothers, four visiting fathers of host-mothers, two visiting sisters of host-mothers, one visiting auntie (actual) of a host-mother, one visiting auntie (distant relative) of host-husband, and one visiting mother of a host-father). The composition of visiting relatives generally indicates that for the majority of the guests, the principal contact person is the immigrant-host mother in New Zealand, therefore typically following a "matrilineal" pattern. 
Table 3.2. Characteristics of Filipino immigrant-host families vis-à-vis their visiting relatives.

\begin{tabular}{|c|c|c|c|c|c|c|}
\hline Family Code* & $\begin{array}{l}\text { Composition } \\
\text { of family }\end{array}$ & $\begin{array}{l}\text { Number of } \\
\text { individual } \\
\text { interviews }\end{array}$ & $\begin{array}{l}\text { Number of } \\
\text { group } \\
\text { interview }\end{array}$ & $\begin{array}{l}\text { Composition of } \\
\text { VR/s }\end{array}$ & $\begin{array}{l}\text { Number of } \\
\text { individual } \\
\text { interviews }\end{array}$ & $\begin{array}{l}\text { Number of } \\
\text { group } \\
\text { interview }\end{array}$ \\
\hline Matutum & $F, M, D, S$ & 4 & 1 & $\begin{array}{l}\text { Parents of host } \\
\text { mother }\end{array}$ & 2 & 1 \\
\hline Banahaw & $\mathrm{H}, \mathrm{W}$ & 2 & 1 & $\begin{array}{l}\text { Mother of host } \\
\text { husband }\end{array}$ & 1 & \\
\hline $\begin{array}{l}\text { Sierra } \\
\text { Madre }\end{array}$ & $\mathrm{M}, \mathrm{S}$ & 2 & 1 & $\begin{array}{l}\text { Father of host } \\
\text { wife }\end{array}$ & 1 & \\
\hline Penablanca & $H, W$ & 2 & 1 & $\begin{array}{l}\text { Auntie of host } \\
\text { husband }\end{array}$ & 1 & \\
\hline Isarog & $F, M, S, S$ & 4 & 1 & $\begin{array}{l}\text { Parents and } \\
\text { sister of host } \\
\text { mother }\end{array}$ & 3 & 1 \\
\hline Makiling & $H, W$ & 2 & 1 & $\begin{array}{l}\text { Mother of host } \\
\text { wife }\end{array}$ & 1 & \\
\hline Apo & $H, W$ & 2 & 1 & $\begin{array}{l}\text { Mother and } \\
\text { auntie of host } \\
\text { wife }\end{array}$ & 2 & 1 \\
\hline Balbalasan & $H, W$ & 2 & 1 & $\begin{array}{l}\text { Mother of host } \\
\text { mother }\end{array}$ & 1 & \\
\hline Arayat & $F, M, D$ & 3 & 1 & $\begin{array}{l}\text { Parents of host } \\
\text { mother }\end{array}$ & 2 & 1 \\
\hline $\begin{array}{l}\text { Guiting- } \\
\text { guiting }\end{array}$ & $H, W$ & 2 & 1 & $\begin{array}{l}\text { Mother and } \\
\text { sister of host } \\
\text { wife }\end{array}$ & 2 & 1 \\
\hline Pulag & $F, M, S$ & 3 & 1 & $\begin{array}{l}\text { Mother of host } \\
\text { mother }\end{array}$ & 1 & \\
\hline \multicolumn{2}{|c|}{$\begin{array}{l}\text { Total interviews with } \\
\text { hosts }\end{array}$} & 28 & 11 & $\begin{array}{l}\text { Total } \\
\text { interviews with } \\
\text { VRs }\end{array}$ & 17 & 5 \\
\hline
\end{tabular}

*Note: pseudonyms used

Legend: F-father; M-mother; D-daughter; S-son; H-husband; W- wife 
Table 3.3. Characteristics of Filipino immigrant-host families vis-à-vis their visiting friends.

\begin{tabular}{|c|c|c|c|c|c|c|}
\hline $\begin{array}{l}\text { Family } \\
\text { Code* }\end{array}$ & $\begin{array}{l}\text { Composition of } \\
\text { family }\end{array}$ & $\begin{array}{c}\text { Number } \\
\text { of } \\
\text { individual } \\
\text { interviews }\end{array}$ & $\begin{array}{l}\text { Number of } \\
\text { group } \\
\text { interview }\end{array}$ & Composition of VF/s & $\begin{array}{c}\text { Number of } \\
\text { individual } \\
\text { interviews }\end{array}$ & $\begin{array}{l}\text { Number of } \\
\text { group } \\
\text { interview }\end{array}$ \\
\hline Mayon & $\mathrm{F}, \mathrm{M}, \mathrm{S}$ & 3 & 1 & Female friend & 1 & \\
\hline Pinatubo & $\begin{array}{l}\text { couple } \\
\text { (Maria and } \\
\text { Leonora)* }\end{array}$ & 2 & 1 & Male friend & 1 & \\
\hline Taal & $\begin{array}{l}\text { Single- } \\
\text { person } \\
\text { household } \\
\text { (Mara)* }\end{array}$ & 1 & & $\begin{array}{l}\text { Female friend } \\
\text { (Clara)* }\end{array}$ & 1 & \\
\hline Ragang & W & 1 & & $\begin{array}{l}\text { Female friend of } \\
\text { host wife and } \\
\text { her mother }\end{array}$ & 2 & 1 \\
\hline Halcon & $\mathrm{H}, \mathrm{W}$ & 2 & 1 & Male friend & 1 & \\
\hline \multicolumn{2}{|c|}{$\begin{array}{l}\text { Total interviews with } \\
\text { hosts }\end{array}$} & 9 & 3 & $\begin{array}{l}\text { Total interviews } \\
\text { with VFs }\end{array}$ & 6 & 1 \\
\hline
\end{tabular}

On the other hand, there were only six visiting friends who were interviewed: three of them were solo travellers (two male and one female); one female VF (accompanied by her relatives who were not interviewed); and one family group (two members composed of a mother and daughter). A common feature of the friendships that may have been formed between the immigrant-hosts and VFs are that the relationships have survived and are maintained over time through visits prior to the migration of the immigrant-hosts. In this case, however, the researcher recognised that other types of friendships may have been formed such as from being pen pals or by way of online websites and other forms of social media. The study acknowledges that friendships may also involve a romantic relationship which apparently was not a characteristic of the social interactions between immigrant-hosts and VFs interviewed for this research. 
Arranging the group interviews with VFs was significantly more difficult than scheduling interviews with the VRs as the composition of the traveling groups within the VFs was more diverse. For instance, the group of visiting friends of the Ragang family who are Filipino-Chinese is composed of five members including: (1) the host-wife's best friend [will be referred to here as "female visiting friend of the Ragang family"] and (2) the best friend's mother [will be referred to as "mother of female visiting friend"]; (3) the best friend's auntie; (4) the auntie's best friend; and (5) another female family friend of her best friend. Convening a group interview that included all five visitors was not possible as they reside in different regions of the Philippines and only two of them were interviewed (for both individual and group interviews). In this case, only the Ragang family's female visiting friend and her mother were interviewed (both individually and as a group) in the province as the rest of the traveling members were based in Manila. As such, within the Ragang family, only the immigrant-host wife in the Ragang family has a significant relationship with only one member of the visiting group, and that was her best friend from college and this therefore led to prioritising the interviews in the province over interviewing the other less significant members of the visiting group in Manila which could have been more easily conducted. Travelling outside Manila was consequently necessary in order to obtain significant information in relation to the social interactions between the primary actors, particularly the immigrant-host wife for the Ragang family and her female visiting friend (her best friend). In addition, interviewing the host-husband of the Ragang family was missed as he was unable to participate in the study due to conflicting shifts at work. It was nonetheless the discretion of the researcher to focus on interviewing participants who might provide richer and deeper insights regarding their social interactions regardless of the inconvenience or distance of travel. For the Taal family, only the female guest (henceforth called "Clara") was interviewed after work as her office was more conveniently located than the family's residence and moreover, a group interview would be quite difficult to arrange as the various family members have very different schedules.

Sixty individual and twenty group interviews were undertaken for this project. The interviews were audio-taped and transcribed verbatim using Express Scribe software. 
An advantage of this software is that it can slow down the replay speed of the audiorecorded interview without changing the voice pitch and that instant play or pause can be done easily with one button. The average duration of the individual interviews for the immigrant-host family members for VRs was 46 minutes, and 30 minutes for the family interview. Immigrant-host families for VFs on average had individual interviews that were 36 minutes in duration while family interviews averaged 20 minutes. The interviews with immigrant-host families who hosted VRs were longer compared to those with the families who hosted VFs. The former group spoke about their relationships that are rooted in kinship, the social obligations between them and their visiting relatives, and these relationships extend to all members of the host family. However, hosting a VF usually is the result of one member of the host family having a significant bond with the VF and this bond may not be uniformly strong or even extend across all members of the host family unit. The variability in number and in the significance of bonds between the host family members and their guest(s) when hosting a VF(s) as opposed to the ties of relatedness between all members of the host family when hosting VR(s) would account for the variance in interview durations.

Individual and family interviews for VRs on average lasted for 28 and 17 minutes, respectively; individual and group interviews for VFs on average were 35 and 15 minutes, respectively. Compared with VRs, the length of the individual interviews with VFs may reflect the amount of information that individuals may need to share with regard to their relationship. As such, the nature of the relationships between relatives are usually considered to be well understood across cultures. While every relationship is unique, there are generally accepted commonalities of family bonds (i.e., between host family and VR) as compared with friendships were participants may need to explain how they became friends. Friendships are relationships that are each unique but may require more explanation to establish the mutual understanding and explain its significance. So, the VF individual interviews are longer. The group interviews for VFs were slightly shorter which indicates the family/group required somewhat less time to describe the significance of their experiences as VFs, and a VF may not have as uniform of bond with all the members of the hosting family. 
Since most of the visiting relatives in this study are directly related to the host-mothers (being daughters/sisters), the researcher recognised that the immigrant-host mother should be the first person in the family to be interviewed in order to have a better understanding of the overall context of the hosting experience. Interviewing the remaining members of the host family members (fathers and adult children) then became easier after interviewing the "principal host." For the families who have toddler(s), focusing on the interview was difficult for parents as young children invariably need frequent attention and care.

At times, there were breaks between interviews or this researcher had to wait while the host family prepared a meal prior to the interview (both in New Zealand and the Philippines). The researcher used any waiting time as an opportunity to request any available photos and souvenirs (whether given as a gift by their guest or bought from a previous visit to the Philippines) that the host/s may have with them. This was an approach suggested by Tung and Ritchie (2011) in order to increase the depth and clarity of the interviews for research participants who are reflecting on their experiences. Collecting photos from the immigrant-host families, especially those who hosted for their VRs, was useful as most grandparent(s) that were interviewed did not have any photos with them of their recent visit to New Zealand (except for one grandfather who showed me a diary of his daily itinerary with his host). In addition, showing the VRs photos taken in New Zealand with the researcher together with their respective host families and allowing them to show their photos and souvenirs of their visit before the interview was a technique that was frequently utilised to establish a rapport and to cultivate a deeper interpersonal relationship with the VRs. However, the use of souvenirs as an approach to facilitate recollection was not possible for the VFs that were interviewed as it was previously mentioned that they were interviewed outside their residence and in a public space.

It also would have been useful to bring a New Zealand map as some of the respondents (even Filipino immigrants in New Zealand) interviewed could not remember many names of places they visited or areas travelled with their host/guest. A New Zealand 
map could have assisted the respondents with their recollections of place and locality. Nonetheless, the study was not about the accuracy of the location but on the meanings of the experiences of the participants and their social interactions. In particular, it is interesting to examine whether there is a stronger emphasis on social interactions of the respondents and less emphasis on the places visited which will be highlighted in subsequent chapters.

The participants were encouraged to speak in a language with which they are comfortable and the respondent's own words were used (regardless whether the expression is in English or Tagalog) to explore the meanings of their social interactions. Interview transcripts in Tagalog have an English translation provided in this study that is more contextual rather than literal as "word for word" translations are awkward at best. This approach will be made to capture the meanings of Filipino language while making sure it makes sense to an English speaking audience. While some nuances may be lost, it ensured that the translated transcripts will be readable, understandable, and insightful (e.g., Batan, 2010). Being consistent with the research paradigm (hermeneutic phenomenology), language plays an essential role where the experience of immigrant-hosts and VFRs is based on interpretation and understanding (Laverty, 2003; Pernecky \& Jamal, 2010).

The unit of analysis is the immigrant-host and VFR social interactions and the host/s can easily identify their guest/s (and vice versa) through their assumed role or relationship (i.e., as parent/s, grandparent/s, sibling/s, or friend/s) or any specific anecdote specifically related to their social interactions. Despite providing pseudonyms for each family, this project acknowledged from the outset that the interviews were neither anonymous nor confidential between the respective members of host families and their guests. This inherent lack of confidentiality within a given family unit was explicitly explained and outlined in the consent forms for host families and VFRs. However, strangers and common acquaintances cannot identify the research participants as they would have to possess detailed and very personal knowledge of the hosts and of their visiting relatives and friends in order to identify any of the 
participants. Participants were cautioned that care should be taken when responding to questions despite the removal of identifying features such as names and other details as their responses could still be attributed to them by their relative(s) and friend(s) participating in this study. This verbal caution acted as a reminder for participants to maintain a certain level of discretion in order to prevent harm being done to the relationships between hosts and guests due to participation in this research.

\subsection{Analytical framework in examining social interactions between immigrant-hosts and VFRs: a thematic analysis approach}

While the main body of this chapter has justified the decisions made regarding the research paradigm and methodology, this section expands upon the development of the analytical strategy. An analytical framework was useful to provide guidance for interpreting the data (Pearce, 2012). A thematic analysis approach (Figure 3.3) was adapted to examine the complexities of immigrant-hosts and VFs and also immigranthosts' and VRs' social interactions. It shows four sample cases (four host and VF or VR pairings) which were worked through for the purpose of analysis. The thematic analysis process is composed of five stages: (1) contextualising; (2) coding; (3) coalescing; (4) conceptualising; and (5) interpreting. The thematic analysis focuses on the core, the social interactions occurring between the first-generation Filipino immigrants in New Zealand as hosts and their relatives and friends from the Philippines as guests.

The analytical framework was developed out of the literature review and is embedded within the conceptual framework on the immigrant-host's( $\left.s^{\prime}\right)$ and VFR's(s') social interactions (discussed earlier on Chapter 2). It depicts the individual and multiple perspectives of the hosts through the individual and group interviews, including hostparent(s) and adult children, together with their visiting relatives or friends encompassing the three stages of time (pre-, during-, and post-visit); underpinning social exchange theory and the theory emotional solidarity and are informed by the values of the shared culture of the immigrant-hosts with their guests which is the 
Filipino culture, while the former group may also be influenced by the culture of their new homeland (New Zealand).

For each case of social interaction between immigrant-hosts and VFs or VRs, the transcribed interviews (or source materials) were read and reread to gain an understanding of each interview and reach a picture of the data as a whole (Marshall \& Rossman, 2006), in this instance, the social interactions between hosts and guests. Being bilingual allowed the researcher to analyse the source materials or interview transcripts regardless whether in Tagalog or English. Each social interactions were analysed across the group of immigrant-host family members or other types of hosts (e.g., single-household and common-law partnership-based relationships) together with their respective VFs or VRs. This study also acknowledged the various types of "hosts" that occur in VF or VR travel and that has shaped the analysis to accommodate the various family members' voices mentioned earlier in section 3.4 .3 , particularly Tables 3.2 and 3.3. 
Figure 3.3. Thematic analysis of the social interactions between immigrant-hosts and VFRs.

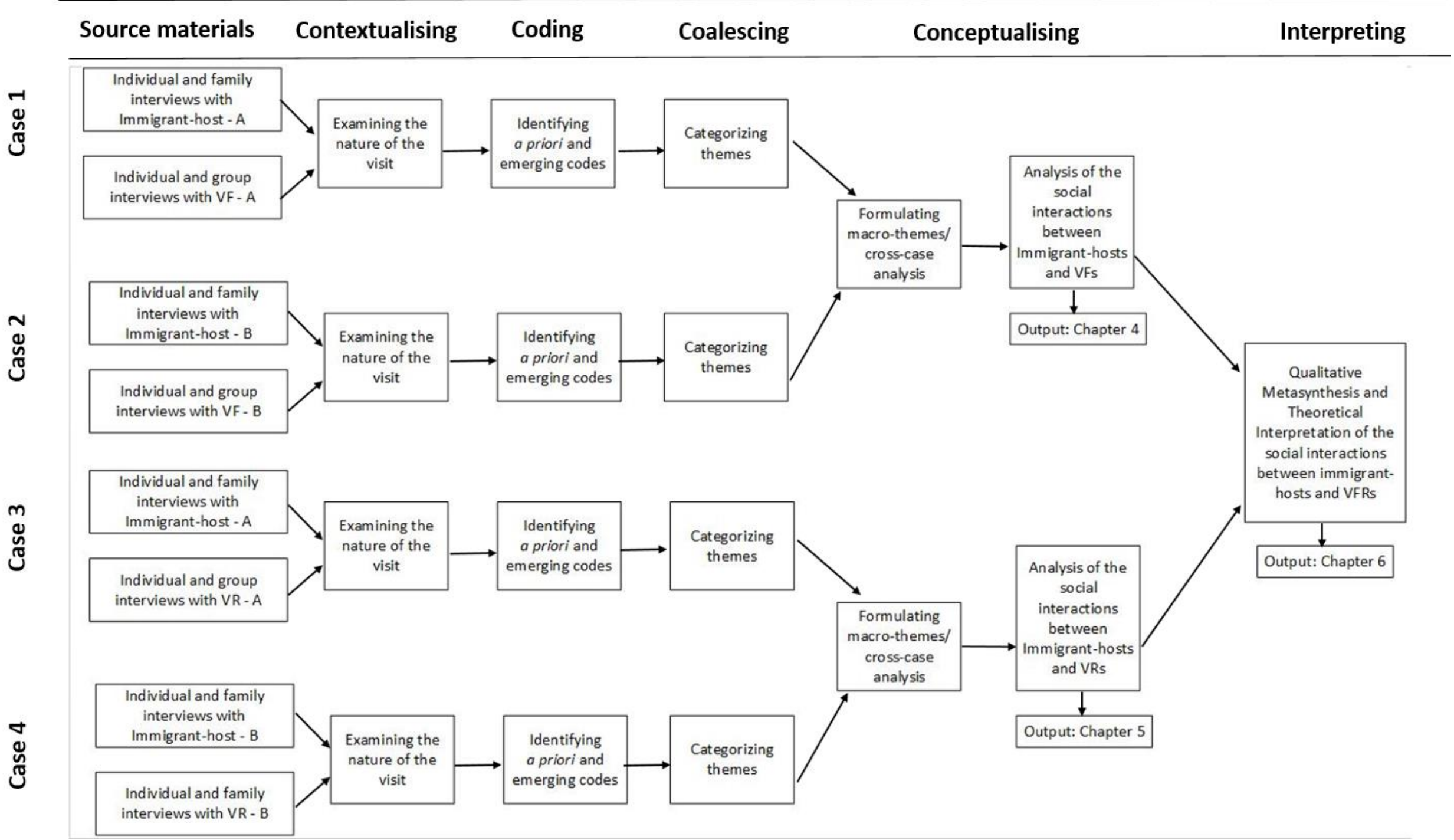


The first phase consisted of contextualising the social interactions between the hosts and guests. Note that the initial state of examination and analysis separates the social interactions of the immigrant-hosts and VFs from those of the immigrant-hosts and VRs due to the following reasons: first, family relationships are different from friendships as the latter are voluntary relationships which contrasts with blood ties with family that tend to persist due to the influence of societal convention and whether a person desires them or not. Hence, the social exchanges and emotional solidarity may be differently viewed by and between immigrant-hosts and VFs as compared against the relationships of immigrant-hosts and VRs; second, research on VFR travel needs to be contextualised as it involves both hosting and visiting of friends or relatives; lastly, each social interaction between immigrant-hosts and VFs or immigrant-hosts and VRs are unique and dynamic. VF or VR travel is a multifaceted phenomenon and it can be difficult to disentangle from the complex aspects of a visit to identify a single factor, activity or trip motivator (Moscardo et al., 2000). Thus, a more integrated approach is needed to advance knowledge about and understanding of this complicated and still growing phenomenon.

Upon analysing each case, the qualitative researcher's first and foremost responsibility is doing justice to each individual case (Patton, 2002, p. 449). The other aspect contained within the phases of analysis was determined by the research questions. While examining the nature of the visit may be descriptive, an innovative approach is that the study presents the different perspectives of the immigrant-hosts and their respective VFs or VRs including their: motivations, expectations, anticipations, duration of the visit, amongst others.

The second phase involved coding the individual and group interviews by examining them line by line and underlining and labelling passages with tentative theme labels. Coding is a way of indexing or categorising the text in order to establish a framework of thematic ideas contained within it (Gibbs, 2007). Passages from the interview text and labels, or theme labels, for each interview are compared with passages and themes between and among all other interviews (Porter \& Cohen, 2013, p. 185) within a 
specific social interaction. Atlas.ti (version 7), a computer-assisted qualitative data management and analysis program, was used to facilitate the coding and subsequent analysis.

Data coding was carried out to identify the a priori codes and emerging codes (Gibson $\&$ Brown, 2009). The a priori codes are general categories that were derived from the theories (pre-specified themes). Then, emerging themes from the transcripts were classified as emerging codes which may surface through the exploration and examination of the data. The a priori and emerging codes were utilised for auto-coding interview transcripts which facilitated the automatic assignment of codes to paragraphs or statements. However, despite the use of auto-coding, all transcripts were carefully read to ensure thorough analysis of the data. Quoted passages could also be classified in more than one category as some statements made by respondents may address more than one theme.

Given the possible range of reasons for undertaking VF or VR travel, other themes may emerge or other forms of meanings may arise from the analysis of the interviews. Nevertheless, these accompanying themes were used to locate and explain what goes on within a specific social interaction in terms of its wider context (see Burawoy, 1998; Sharp, 1982), particularly within the host(s)-guest(s) relationship. Memo writing is another fundamental process for empirical codes as the memos provide a bank of ideas which can be revisited and help map out the emerging theory (Goulding \& Saren, 2010). In total, there were 80 transcripts in .Doc format uploaded to Atlas.ti 7 for analysis which, when compiled, consisted of 1,398 single-spaced pages of transcribed interviews.

The third phase of analysis involved coalescing codes in order to develop and categorise the themes. At this stage, a priori and emerging codes were coalesced to bring together components or fragments of ideas which often are meaningless when viewed alone. Codes that emerged from the interviews are pieced together to form a comprehensive picture of the social interactions (e.g., Aronson, 1994). For example, when examining a 
particular immigrant-host family and VF social interaction, the activities done by the host such (an a priori code: activities of the host) as treating a friend by taking them out or by accommodating them in the residence pertains to their social obligations to their guest (another a priori code) which when examined further is connected to the host's notion of cultural norms related to "hospitality" (an emerging code). Note that while codes are being coalesced for the immigrant-hosts, a similar mirror pattern occurs with their respective VFs or VRs.

Quotations from the transcripts are thereby extracted and then incorporated into the analytical framework for each social interaction. The identified codes prior to analysis are derived from the theories that were used to understand the host(s)-guest(s) interactions. In this case, the cultural norm of hospitality is a social exchange offered by a host to a VF. A hermeneutic study allows the researcher to draw upon an eclectic range of theories from contextual/perspectivist/inter-subjective outlooks to interpret the phenomenon being investigated (Jamal \& Hollinshead, 2001) and analysis begins by reading each transcript several times to get the perspective of the subjects and a good sense of each interview as a whole.

Moving to a higher level of analysis, the fourth phase involved conceptualising the themes and the macro-themes which evolved from categorising the micro-themes while considering the specific research questions related to immigrant-host's(s') and $V F^{\prime} s\left(s^{\prime}\right) / V R^{\prime} s\left(s^{\prime}\right)$ social interactions in relation to the social exchanges, emotional solidarity, and the cultural context of their interactions. All themes of the social interactions that were contextualised and coalesced were formulated to develop the macro-themes. Going back to the earlier example which generates codes such as (1) activities of the host, (2) obligations, (3) cultural norms, and (4) hospitality - these were incorporated to form the social exchanges between the hosts and VFs. However, the social exchanges between them are rooted in their past interactions or the historical origin of their friendships. During this stage, cross-case comparison was made and found that such acts of hospitality stem from their social exchanges as friends as these actors were maintaining such relationship across distances. Therefore, the macro- 
theme: past interactions and social exchange was developed at the conceptualisation stage. This reinforces the view of Corbin and Strauss (2008, p. 52) who stated:

The more one moves up the conceptual ladder, the broader and more explanatory the concepts become, yet as they move toward greater abstraction, concepts, while perhaps gaining in explanatory power, begin to lose some of their specificity. However, if the conceptual pyramid is carefully crafted, the higher-level concepts will rest on a solid foundation of lower-level concepts.

When compared with most quantitative research which may take the individual as the unit of analysis, qualitative research can accommodate multiple perspectives and can better analyse families and internal relationships as units to enable richer accounts of lived family experiences (Handel, 1992) and interpret the meanings attached to the range of host-guest interactions. Furthermore, it was only during the conceptualisation phase when interviews in Tagalog or Taglish were translated into English in order to consistently present them and which forms the basis of Chapters Four (Analysis of the social interactions between immigrant-hosts and VFs) and Chapter Five (Analysis of the social interactions between immigrant-hosts and VRs). The basic assumption when translating interview transcripts at the higher level of analysis is that the coding process at the lower level of analysis remains faithful to the research participants' intentions. The translator's task is to remain faithful to the "original" without overturning the privileged status of English language (Temple, 2005).

The final phase is the interpretive stage. In this stage, the act of analysis could be viewed as being similar to the role of a researcher when reading the pieces of qualitative research and deciding how useful it is when examining the social interactions of the immigrant-hosts and VFRs as phenomena. Interpretation means attaching significance to what was found, making sense of the findings, offering explanations, drawing conclusions, extrapolating lessons, and making inferences (Patton, 2002, p. 480). In the case of qualitative metasynthesis, it is suggested that themes and concepts are compared from one situation to another are transferable across other concepts (Thomas \& Harden, 2008) and it involves bringing together the results of Chapters Four and Five. The interpretation of the phenomenon is a 
productive process that sets forth the multiple meanings of social interactions which illuminate and throws light on experience with the intention of bringing out and refining the meanings of the social interactions of the immigrant-hosts and VFs/VRs (see Corbin \& Strauss, 2008; Denzin \& Lincoln, 1998). As in qualitative research, this study of host(s)-guest(s) interaction in the context of VFR travel recognises that familial relationships and friendship groups entail social realities. The last section of this chapter discusses the strengths and limitations of the methodology.

\subsection{Strengths and limitations of the methodology}

This section reflects the methodological strengths and limitations when conducting studies related to the social interactions of immigrant-hosts and VFRs. In order to highlight the strengths of the methodology, it focuses on the quality of interviews using the indicators developed by Kvale (2007) and Kvale and Brinkmann (2009): (a) the extent of spontaneous, rich, specific, and relevant answers from the interview; (b) the extent of short interviewer questions and longer interviewee answers; (c) the degree to which the interviewer follows up and clarifies the meanings of the relevant aspects of the answers; (d) to a large extent, the interview providing rich texts for further interpretation; (e) the interviewer attempting to verify his or her interpretations of the subject's answers over the course of the interview; and (f) the interview being "selfreported"; a self-reliant story that hardly requires additional explanations. When examining the duration of individual interviews with the different members of the immigrant-host family, the wealth of information that was offered by the host-mothers for visiting relatives and their individual interviews had an average length of 57 minutes. The average interview with host-fathers and adult children (both sons and daughters) ranged between 36 to 38 minutes. On the other hand, the average length of individual interview with visiting relatives (regardless of gender) was 42 minutes.

As the researcher had a previous social connection with some Filipino immigrant-host families (indicated in section 3.4.1), there is a discernible increase in level of spontaneity, openness and sincerity gathered from those interviews - regardless of the role of the participant within the family (host parent(s) or children). Most of the 
families kindly invited me to share a meal with them in their home, either before or after the interviews, which I did not decline as a sign of acceptance and consideration of their kindness for participating in the study. The offer of a meal prior to the interview was an ideal opportunity to establish a rapport with a family, if not previously known by them or, in the instances where I knew one or two family members this was an opportunity to make the acquaintance of the remaining family members. As the intended purpose of my visit and the nature of my study were discussed at some point during the meal, it also allowed me to get comfortable with the respondents and for them to feel comfortable and accepting of one another in collectively responding to questions about shared personal experiences. Certainly, the level of interaction or relationship that exists between the researcher and the participants significantly determines the quality of the data obtained (Pe-Pua, 2006).

Overall, the participants were approachable and interacting with them was quite effortless as they share the same cultural background and language with the author which facilitated in building a rapport with them. This situation encouraged them to be more open and forthcoming in their responses. Such significant advantages allowed the researcher to enter into a meaningful discourse with the VFs and VRs without the impediments and typical barriers to achieving a quality interview that a typical Western researcher could face: initial shyness, excessive respect and deference, reserved behaviour and hesitancy to reply in a direct and straightforward manner for fear of offending, and hesitancy or embarrassment to be interviewed for fear that their English is poor. In conversing with the Filipino immigrant-hosts or the VFRs, the language of the participants was used as the language of the research at all times as it is through their own mother tongue that they can truly express their innermost sentiments, ideas, perceptions, and attitudes (Pe-Pua, 2006).

Being a "cultural insider" gave the researcher the ability to ask more meaningful questions and to more fully read non-verbal cues, and most importantly, be able to project a more truthful, authentic understanding of the culture under study (Merriam et al., 2001), in this case, Filipino culture that is situated in the multicultural setting of 
New Zealand. The researcher's previous profession as a conservationist involved interviewing indigenous people and coastal communities from various regions of the Philippines and, as a result, a natural facility of approaching and entering into discussions on a broad range of topics in a non-threatening and supportive manner was gained and put into practice for this study.

Even when dealing with adult children, particularly for those who were raised in New Zealand (e.g., the immigrant-host daughter for the Arayat family and immigrant-host sons for the Isarog family) who are more comfortable speaking English rather than in their parents' native tongue, the researcher shares the same ethnicity, language, and cultural identity of their parents and their respective VRs, allowing both parties (the researcher and the participant) to quickly become more engaged in a meaningful discourse. With regard to the other adult children who can fluently speak Tagalog, their use of verbal expressions po or opo when speaking were noticeable (this is an expression of individual's respect for an elder or a person of high standing, regardless of gender and is uttered before and after every sentence) although the researcher did not expect or demand to be addressed in this manner during the course of the interview as the researcher treated each and every individual member of the family as an equal (Church, 1987).

A non-Filipino would no doubt approach the research differently and the expected "bias" of a (partial) insider would be replaced with the perspective of someone (a socalled "outsider") who does not have the same cultural connections (e.g., Pe-Pua, 2006). However, a researcher may be an outsider to the subculture under study (e.g., immigrant-host children who grew up in New Zealand) but may be considered an insider due to a shared language and culture (Enriquez, 1993). In this case, the researcher shares the native culture and ethnicity of origin of the immigrant-host children and their parents. The Filipino culture is a commonality that the researcher shares with the parents and even their adult children. However, as the researcher began studying in Wellington in 2011 and was therefore unfamiliar with some aspects of New Zealand, a culture within which these adult children are immersed. 
The breadth and depth of individual interviews varies depending on the relationship of the hosts and VFs/VRs as well as the purpose, duration, and obligations while hosting/visiting. Since the principal contact person for most of the VRs is the immigrant-host mother (therefore, directly related as daughter to their visiting parents), the higher quality and more detailed responses usually came from the immigrant-host mother as compared to the remaining family members who did not share the same level of past interactions or hosting responsibilities. In addition, the use of photographs, souvenirs, and diaries during the interviews with the VRs were also useful in improving the quality of responses and in providing richer information (Figure 3.4) and due consideration when interviewing elderly participants was also made by Holstein and Gubrium (2003).

\section{Figure 3.4. Souvenirs bought by visiting relatives during their recent trip to New Zealand.}
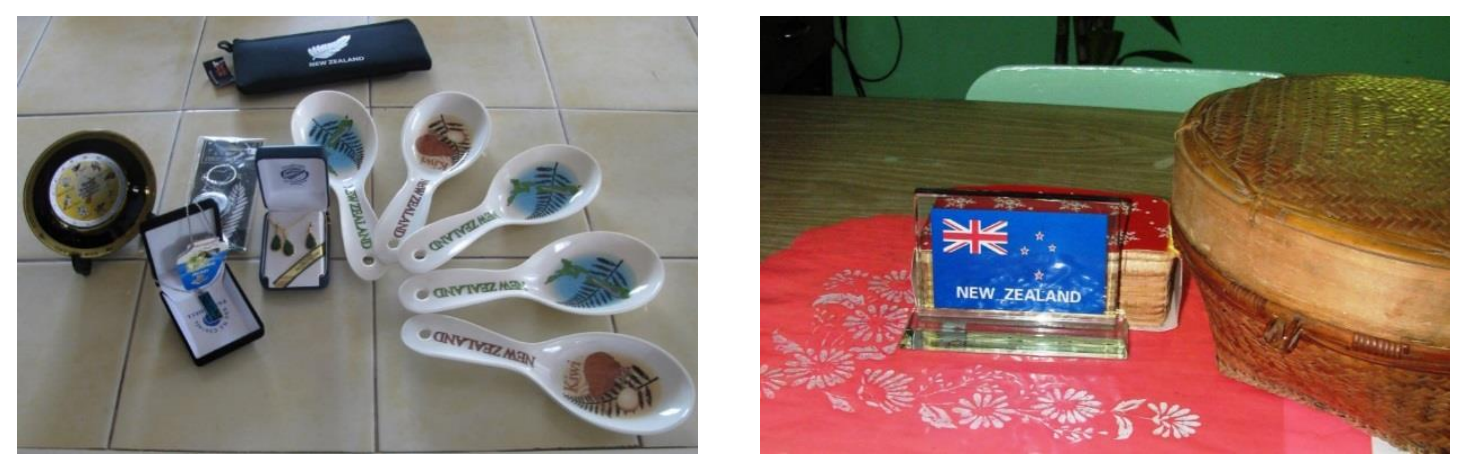

There is a gendered dimension in hosting for VFRs which will be discussed in Chapters Four and Five. Hosting is for the most part a gender specific activity of immigrant-host mothers and to a lesser extent, the remaining family members as they accommodate their visiting relatives within their residence. On the other hand, Filipino men, particularly the immigrant-host fathers or husbands, were less expressive during individual and group interviews, except for host-husbands in the Penablanca and Banahaw families as they were the principal contact persons of their family's VRs (hosting their auntie and mother, respectively). 
The length of the interviews with the hosts is relatively longer than the VFRs. Hosting is by custom a major responsibility and a Filipino family who has much to prepare in the anticipation of their relative's(s') visit, particularly when the guest(s) is/are staying in their residence. In particular, most of the visiting relatives were grandparents. As such, they are highly respected and have special needs due to their age and role within the family as compared to visiting friends who are mostly professionals and are largely independent and can be left to their own devices for extended periods of time. The duration of the interview also depends on the level of care the VR requires or, on the obligation of the host to the VR, and vice versa.

The differences in affinity between adult children and their visiting relatives, particularly those who grew up in the Philippines versus those who grew up in New Zealand is evident from the differences in their responses during the individual interviews. In both cases they were open and candid in responding to the individual and group interviews, but there was more information provided by the participant children who grew up in the Philippines. To get the bigger picture of the relationship between immigrant-host children with their VFRs, informal questions were asked about their return visits to the Philippines, in order to examine the significance of their social relationships with their Filipino relatives determined from their recounting of the trips that they made to their parents' former homeland (e.g., Duval, 2003; Huong \& King, 2002).

However, there are also limitations when conducting this type of research specifically in the use of photo-elicitation and the achievement of a whole-family perspective, in particular with an immigrant-host family. While photo-elicitation triggers memories for the participants (Cederholm, 2004), requiring the immigrant-host families to provide photos of them together with their VFRs presented them with a burden despite an early request from the researcher (as reflected in the pre-interview information sheet which is found in Appendix 3). Perhaps it was time consuming and/or confusing, particularly for families that had to sort through their photos and it may have been especially difficult for those who have hosted their relatives on multiple occasions 
(return visits) to pick out the photos from the most recent visit. Conceivably, a deeper reflection may be needed as to whether succeeding visits of the VFRs produce less photographs for both the hosts and guests as there may be a decrease in the desire or need to capture moments of a similar visit that has been made previously.

For immigrant-host families who did not have time to provide photos of the visit, they have entrusted the author to download their photos from Facebook and bring them when their family or friends were interviewed. Nonetheless, the photos that were copied and brought to the Philippines were useful in gaining the trust of the VRs as the researcher had a significant souvenir of their visit with their host-family in New Zealand. Interviewing VRs in the Philippines was easier to conduct despite interviewing many aged (grandparents) VRs who may be technologically-challenged (e.g., unable to produce and store digital photos). Despite the technological challenges experienced by aged participants, they frequently had more time to accommodate the researcher and they readily bring out their souvenirs and/or diaries from their visit. This researcher also observed that members of the family of the VRs assisted in preparing a meal for the researcher who was treated as guest. This scenario was not observed in interviewing the VFs as they were not interviewed in their homes.

The complexity of immigrant-host families was a common impediment to achieving a whole-family perspective derived from the perspectives of at least one parent and one child (Schänzel et al., 2005). A commonality observed among the participants is that most immigrant-host families are younger and are required to frequently tend to their children that are below 18 years old. This study acknowledges excluding the voices of younger family members, particularly in missing the input of seven children who have hosted for their VRs (four high school and three primary school children from five different immigrant-host families) and only one child (primary school) who hosted his parents' VFs. From an ethical point of view, this author also felt it to be unsuitable or a potential ethical risk should there be interviews with children or teenage youth with a lone adult male researcher. Also, this researcher does not have any prior experience in 
conducting interviews with minor children as compared to extensive experience with interviewing adults.

Overall, the quality of the interviews with visiting relatives provides richer insights than the interviews conducted with visiting friends because the former group were interviewed in their home and they were free from other commitments and in a relaxed environment (Astedt-Kurki, Paavilainen, \& Lehti, 2001). As mentioned previously in the data collection section, interviews with VFs were held outside of their residence in cafés or restaurants which was neither conducive to reflection nor to the providing of detailed and nuanced responses due to the distractions of the inherent hustle and bustle of public spaces. Nonetheless, the openness of the VFs is expressed by their receptiveness during the interviews and the interviews yielded valuable data.

\subsection{Conclusion}

This chapter has outlined the philosophical (interpretive) and research paradigm (hermeneutic phenomenology) that serves as the foundation for this study. There has been difficulty in identifying or locating the theoretical paradigms informing VFR travel research as previous studies are informed by positivist approaches. Equally, there is an absence of articles examining VFR travel research associated with using qualitative methods that reflect the perspectives of both the hosts and guests resulting from their social interactions. The practical steps engaged in (in terms of participant recruitment, data collection, ethical considerations, analyses and evaluation) have been detailed. This chapter has provided an original contribution towards considering the methodological and ethical implications of studying VFR travel in the context of hostguest interactions (see Capistrano, 2013). Dealing with pairs of hosts and guests at the micro-level has certain methodological and ethical implication as these participants have specific personal knowledge about their relationship.

A strength of this research lies in the analysis; the data collection of the social interactions between the immigrant-hosts and VFs and the immigrant-hosts and VRs, related to both the hosts and their guests encompasses time and distance as the 
research participants were all interviewed after their face-to-face interaction in New Zealand (and not at the during-visit phase). Qualitative methods could complement the existing quantitative-oriented epistemologies and methods used in VFR travel studies, particularly when examining the meanings and interpretations of the hostguest social interaction and the cultural context embedded in their relationship.

Similarly, the analytical framework captures the multiple-perspectives of the immigrant-hosts and VFs/VRs for examining the cultural context of their social exchanges, emotional solidarity, and the interpretation of the meanings of their social interactions. The analytical framework provides a guide for analysing the data and enabled in structuring the presentation of the results and analysis. The associated findings will be presented in the analysis section and are outlined in the next three chapters. Chapter Four presents the findings on the analysis of the social interactions between immigrant-hosts and VFs, and Chapter Five discusses the analysis of the social interactions between immigrant-hosts and VRs. Chapter Six provides a theoretical understanding of the social interactions between immigrant-hosts and VFs and between immigrant-hosts and VRs. 


\title{
CHAPTER 4 - Analysis of the social interactions between immigrant-hosts and visiting friends
}

\author{
Hospitality is shown to friends because "liking and affection \\ are inherent in friendship; the liking produces a wish for the \\ friends' company (as distinct from company in general), the \\ affection a desire to please them." (Telfer, 1996, p. 96; cited \\ in O'Gorman, 2010, p. 23)
}

\subsection{Introduction}

As the above quotation would suggest, this chapter is focused on the immigranthost's $\left(s^{\prime}\right)$ and visiting friend's(s') social interactions, specifically in the context of Filipino immigrants in New Zealand and their visiting friends from the Philippines. In particular, this chapter discusses how social interactions occur through social exchanges and emotional solidarity within New Zealand, particularly for the immigranthosts and VFs who may still share common beliefs and behaviours about their friendships and culture as Filipinos despite the situational and cultural influences of a new homeland upon the immigrant-hosts. Examination of the immigrant-host's(s') and VF's(s') social interactions reveals that they are multi-faceted and layered, which generates a range of themes encompassing time, persons involved, places, and culture. Therefore, this is evidence that the immigrant-host's $\left(s^{\prime}\right)$ and $V F^{\prime} s\left(s^{\prime}\right)$ social interactions persist and remain quite complex despite their separation over time and space.

This chapter addresses the main research question in the context of immigranthost's(s')-VF's(s') social interactions: “How are social interactions between immigranthosts and their visiting friends understood and interpreted by them?" This question is addressed by answering the four supplementary questions: (1) What is the nature of social interactions between the immigrant-hosts and VFs? (2) What are the social exchanges between the immigrant-hosts and their VFs? (3) How do social interactions reflect emotional solidarity between the immigrant-hosts and VFs as expressed by their shared beliefs and behaviours? and finally (4) How does culture shape social interactions between the immigrant-hosts and VFs? 
This chapter explores the immigrant-host's(s') and VF's( $\left.s^{\prime}\right)$ social interactions in terms of two theoretical frameworks: social exchange theory and the theory of emotional solidarity. These two theories provide a conceptual basis for examining the phenomenon and in understanding the complexities of the relationships between immigrant-hosts and VFs within a cultural context. Such an approach offers an innovative way of combining these two theories that are not always considered in relation to culture, thereby presenting distinct ways of understanding social interactions in the context of the cultural identities, exchanges, beliefs, and behaviours of first-generation migrants in New Zealand and their visiting friends from the Philippines.

The structure of this chapter parallels the specific research questions presented earlier. The first supplementary research question is addressed in section 4.2 as it provides background about the nature of the visits between immigrant-hosts and VFs. The social interactions between them are also reflected by the multi-destination trips taken by some VFs that may either be influenced by their individual motivations, their personal networks, or those of their respective hosts (discussed in section 4.3). Additionally, a better understanding of the social exchanges, emotional solidarity, and the cultural beliefs and behaviours of the immigrant-host's(s') and VF's(s') social interactions are revealed and discussed in detail.

The second supplementary research question which addresses social exchange is discussed in section 4.4. Both hosting and visiting are a product of past social exchanges between immigrant-hosts and VFs. Friendships that exist between the immigrant-hosts and VFs are based on the nature of their past interactions which result in on-going and intertwined mutual exchanges such as the paying of a visit to a friend's home which naturally would entail the hosting of visiting friends. The third supplementary research question underpins the role of emotional solidarity and is discussed in section 4.5 (Emotional solidarity: Connectedness in togetherness). It describes how social interactions are strengthened through a friend's co-presence. The fourth supplementary research question is related to cultural beliefs and behaviours on 
hospitality and is addressed in section 4.6. The main research question for this chapter is addressed in section 4.7 (Meanings of the visit among friends) as immigrant-hosts and VFs interpret their social interactions. All the themes from sections 4.1 to 4.6 which address the various research questions are brought together in the concluding section of this chapter (section 4.8).

\subsection{The nature of the visit}

As for addressing the first supplementary question: "What is the nature of social interactions between the immigrant-hosts and VFs?", Table 4.1 displays the pseudonyms of the different immigrant-host families interviewed in New Zealand, including the duration of the visit of their respective VFs, the total duration of their visit in New Zealand and the general characteristics of the immigrant-hosts and VFs together with their stated motivation to host or visit from their own individual perspectives. However, interviews on occasion reveal other motivations that emerge from the analysis. The residency of immigrant-host families in New Zealand ranges from two to fifteen years while the length of stay of the VFs with the immigrant-hosts ranges from three to ten days. Whereas the total duration of stay of Filipino VFs in New Zealand ranges from one to three weeks given that most of the VFs interviewed are professionals who had other work commitments or obligations in New Zealand which occupied the remainder of their stay over and above the duration of the visit with their host(s). Coincidentally, all the guests of the immigrant-host families from the Philippines participating in this study were first-time visitors to New Zealand and are active professionals, often making it difficult to arrange for an interview (previously discussed in the methodology section in Chapter 3).

Discussions on what defines VFR travel were presented earlier in Chapter 2 and include the purpose of the visit, accommodation used, and motivations. However, a wider debate on motivations in the context of visiting a friend may be needed as they are multi-faceted and frequently involving not only the guest(s) but also member/s of the 
hosting family/party. The range of responses from immigrant-hosts and VFs reflects the complexity of social interactions in the context of hosting for visiting friends.

Prior to analysing the social interactions of the immigrant-hosts and VFs, it was necessary to establish the nature of the visit by investigating the following: (a) anticipation and planning; (b) purpose of the hosting/visit; (c) motivations; and (d) expectations. As several actors were engaged, the dynamics for each social interaction were difficult to capture given the different responses of the hosts and guests in terms of the purpose and motivations for hosting and visiting compared with the nature of the social interactions between immigrant-hosts and VRs presented in Chapter 5 . In this case, the perspectives of the immigrant-hosts are presented first followed by those of the guests.

In particular, the pre-visit phase entailed anticipation and planning which was usually carried out by the principal host within a specific family unit. As friends from the Philippines expressed their plans to visit New Zealand, immigrant-host families took advantage of the opportunity to reconnect and invited their friends to visit.

I think he sent me an email saying that he is going to New Zealand [to visit his relatives in Auckland]. Maria, female immigrant-host, individual interview, Pinatubo family

I think it was only two days before I found out that she was coming [attending the wedding]. I urgently sent her an e-mail and gave my other contact details in case she experienced any issues in the airport. I oriented her as to what to do [and expect] in the airport. Immigrant-host mother, Mayon family, individual interview 
Table 4.1. Biographical information on the immigrant-host families vis-à-vis their friend's(s') visit.

\begin{tabular}{|c|c|c|c|c|c|c|c|c|}
\hline $\begin{array}{l}\text { Immigrant- } \\
\text { host } \\
\text { Family } \\
\text { Name }\end{array}$ & $\begin{array}{l}\text { Years of } \\
\text { residency }\end{array}$ & $\begin{array}{l}\text { Composition of } \\
\text { immigrant-host } \\
\text { family }\end{array}$ & $\begin{array}{l}\text { Motivation of the } \\
\text { Immigrant-host }\end{array}$ & $\begin{array}{l}\text { Composition of } \\
\text { visiting friends }\end{array}$ & Motivation of the VF & $\begin{array}{l}\text { Duration of } \\
\text { friend's(s') } \\
\text { visit with the } \\
\text { host family }\end{array}$ & $\begin{array}{c}\text { Total } \\
\text { duration of } \\
\text { stay of VF(s) } \\
\text { in New } \\
\text { Zealand }\end{array}$ & $\begin{array}{c}\text { First-time visit of } \\
\text { the VF to New } \\
\text { Zealand } \\
\text { (Yes or No) }\end{array}$ \\
\hline \multirow[t]{2}{*}{ Halcon } & \multirow[t]{2}{*}{2} & $\begin{array}{l}\text { Immigrant-host } \\
\text { husband }\end{array}$ & $\begin{array}{l}\text { invited guest to visit } \\
\text { New Zealand }\end{array}$ & \multirow{2}{*}{$\begin{array}{l}\text { Male visiting friend } \\
\text { of immigrant-host } \\
\text { husband }\end{array}$} & \multirow{2}{*}{$\begin{array}{l}\text { - attended a conference } \\
\text { - visit relatives of girlfriend } \\
\text { - visit friend/s }\end{array}$} & \multirow[t]{2}{*}{3 days } & \multirow[t]{2}{*}{2 weeks } & \multirow[t]{2}{*}{ Yes } \\
\hline & & $\begin{array}{l}\text { Immigrant-host } \\
\text { wife }\end{array}$ & $\begin{array}{l}\text { assist husband in } \\
\text { hosting }\end{array}$ & & & & & \\
\hline \multirow[t]{3}{*}{ Mayon } & \multirow[t]{3}{*}{$>15$} & $\begin{array}{l}\text { Immigrant-host } \\
\text { father }\end{array}$ & $\begin{array}{l}\text { invited guest to visit } \\
\text { New Zealand }\end{array}$ & \multirow{3}{*}{$\begin{array}{l}\text { Female visiting } \\
\text { friend of immigrant- } \\
\text { host parents }\end{array}$} & \multirow{3}{*}{$\begin{array}{l}\text { - attended a wedding of } \\
\text { another friend } \\
\text { - visit friend/s }\end{array}$} & \multirow[t]{3}{*}{1 week } & \multirow[t]{3}{*}{3 weeks } & \multirow[t]{3}{*}{ Yes } \\
\hline & & $\begin{array}{l}\text { Immigrant-host } \\
\text { mother }\end{array}$ & $\begin{array}{l}\text { invite guest to visit } \\
\text { New Zealand }\end{array}$ & & & & & \\
\hline & & Immigrant-host son & $\begin{array}{l}\text { assisted parents in } \\
\text { hosting }\end{array}$ & & & & & \\
\hline \multirow[t]{2}{*}{ Pinatubo } & \multirow[t]{2}{*}{3} & $\begin{array}{l}\text { Maria (lesbian } \\
\text { couple) }\end{array}$ & $\begin{array}{l}\text { invited guest to visit } \\
\text { New Zealand }\end{array}$ & \multirow[t]{2}{*}{$\begin{array}{l}\text { Male visiting friend } \\
\text { of Maria }\end{array}$} & \multirow[t]{2}{*}{$\begin{array}{l}\text { - holiday } \\
\text { - visited relatives and Maria }\end{array}$} & \multirow[t]{2}{*}{3 days } & \multirow[t]{2}{*}{3 weeks } & \multirow[t]{2}{*}{ Yes } \\
\hline & & $\begin{array}{l}\text { Leonora } \\
\text { (lesbian couple) }\end{array}$ & $\begin{array}{l}\text { assisted partner in } \\
\text { hosting }\end{array}$ & & & & & \\
\hline \multirow[t]{2}{*}{ Ragang } & \multirow[t]{2}{*}{4} & \multirow[t]{2}{*}{$\begin{array}{l}\text { Immigrant-host } \\
\text { wife }\end{array}$} & \multirow[t]{2}{*}{$\begin{array}{l}\text { assisted best friend } \\
\text { in traveling within } \\
\text { New Zealand }\end{array}$} & $\begin{array}{l}\text { Female best friend } \\
\text { of immigrant-host } \\
\text { wife }\end{array}$ & $\begin{array}{l}\text { - holiday } \\
\text { - visit relatives } \\
\text { - visit best friend }\end{array}$ & \multirow[t]{2}{*}{1 week } & \multirow[t]{2}{*}{10 days } & \multirow[t]{2}{*}{ Yes } \\
\hline & & & & $\begin{array}{l}\text { Mother of female } \\
\text { best friend }\end{array}$ & $\begin{array}{l}\text { - holiday } \\
\text { - visit relatives }\end{array}$ & & & \\
\hline Taal & 3 & Female host (Mara) & $\begin{array}{l}\text { assisted former } \\
\text { partner's relatives } \\
\text { who are on holiday } \\
\text { to New Zealand }\end{array}$ & $\begin{array}{l}\text { Female visiting } \\
\text { friend (Clara) }\end{array}$ & $\begin{array}{l}\text { - holiday } \\
\text { - visit relatives and friends }\end{array}$ & 10 days & 10 days & Yes \\
\hline
\end{tabular}


However, as one would expect in the pre-visit phase, some members within the immigrant-host family have less involvement and interaction with the guests due to a lack of social interconnectedness. Therefore, other members of the household hosting VFs often relate passive notions of being aware but having little to no obligations in the pre-visit phase as compared with the principal contact person/host of the guest:

Nothing. It is all about Maria coordinating that. She bought the ticket and told me that Kuya ${ }^{7}$ was visiting. Leonora, female immigrant-host, Pinatubo family, individual interview

As it was being planned, no, ah it was more my former partner. So, I wasn't involved in that. Mara, female immigrant-host, Taal family, individual interview

When he is about to visit? It was our guest and my husband who communicate with one another. Immigrant-host wife, Halcon family, individual interview

From the perspective of the guests, other components underpinning the nature of the visit cut across a wide spectrum of intentions such as determining the purpose of the visit and motivations:

I just wanted to see my cousin and wanted to see New Zealand, my family loves traveling and we've never been there. Clara, female visiting friend of Taal family

To visit New Zealand; I knew that New Zealand is a very beautiful country, right? I see that on TV and read about them in the Book of Knowledge when I was in primary school. The pictures of New Zealand were beautiful. So, since then, I wanted to go there. And then, I knew some places that I really wanted to visit like Rotorua and its geysers. I wanted to visit those and then I wanted to see what the Maori look like and their culture. Male visiting friend of Pinatubo family

Meanwhile, guests of the Mayon and Halcon families have other individual purposes or motivations for visiting New Zealand other than visiting with their respective host(s), which form the main context of the interviews of this study. The female visiting friend of the Mayon family was also motivated to visit New Zealand as she had an obligation

\footnotetext{
${ }^{7}$ A Filipino term used as a sign of respect to an older male relative such as a brother, cousin, or family friend. The word may also be used to call a man older than them with respect, even if they are not relatives.
} 
to participate in a $25^{\text {th }}$ wedding anniversary celebration as she was a primary sponsor (or godmother) of another friend's wedding and they also reside in Auckland. Similarly, the purpose of the trip of the visiting friend of the Halcon family was to attend a professional conference in Auckland but, he decided to make an ancillary trip to Wellington to visit his best friend and family. The Ragang family's visitor also selfidentified multiple motivations to travel to New Zealand:

Because I have two reasons to go there - to visit a new country and at the same time visit family and friends. Female visiting friend of Ragang family

This underscores that multiple motivations which frequently incite the visiting of friends and they often have other reasons for traveling to New Zealand, some of them being work-related and/or other social obligations. As a consequence, the respective immigrant-host families seize upon the knowledge of the trip made by their friend(s) from the Philippines to New Zealand as an opportunity to extend an invitation to their potential guest(s) and suggest that they spend a portion of their trip with them to maximise their travel to New Zealand and re-acquaint, maintain, and/or renew their friendship ties:

Actually, I told him that if he visits New Zealand, he should drop by Wellington. Although it wasn't really part of his plan to come to this city. From Auckland, he wanted to go straight to Christchurch. While in Auckland, I know he attended a conference. I told him, come and visit us to see Wellington and see what's happening in our lives here, which he accepted to do. Immigrant-host father, Halcon family, individual interview

To see him. We are very good friends. I paid his travel from Auckland to Wellington because his primary purpose was to visit his relatives in Auckland and he told me that he was in New Zealand. So, I said, "Why not come and visit me here?" [...] Even before he came to New Zealand, he already asked me how I would feel if he would visit and I really encouraged him to come and stay with me. Maria, female immigrant-host, Pinatubo family, individual interview

We also would like to invite her to show New Zealand, particularly Wellington. Since Auckland is far from here, we wanted her to visit us since it's her priority to see us being her friends. Immigrant-host father, Mayon family 
Meanwhile, the Ragang and Taal families' primary motivation for hosting was the feeling of being compelled due to their guests' need for accommodation. As the Ragang family felt unable to house a large group of visitors within their home, the immigranthost mother for the Ragang family turned down her friend's request to stay in their residence and instead offered a recommendation:

Around November or December, she communicated with me through text or Facebook. I told her I would love her to stay at my place. However, I only have three rooms and since she has her uncle [in New Zealand], he may be offended if he finds out that she stayed with others. My friend said that she is not really close with her uncle even if he is the brother of her mother. He only goes home to [name of province] every now and then. It has been fifteen years and that his attitude may have changed and may no longer reflect being Filipino. Immigrant-host wife, Ragang family, individual interview

The preceding account indicates that while the guest has an immediate relative in New Zealand, she would rather choose to stay with the Ragang family, even if the living quarters would be minimal. However, the socio-economic status of the Ragang family's guest is more affluent and the Ragangs are concerned that they would not be able to properly host them in their small home. So, the immigrant-host mother convinced their guest to stay with a nearby uncle who enjoys a higher standard of living and has a larger home. The guest agrees to the Ragang's suggestion to reside with their uncle despite their loss of ties with him since the uncle has been living overseas for many years.

On the other hand, the female host (pseudonym: Mara) felt compelled to conform (or pakikibagay in Filipino terms) and agree to accepting guests within their home out of respect and consideration to her cohabitating former partner's desire to host guests:

It was a surprise visit. And they wanted to visit my former partner. So, instead of them, ah renting out, or staying in a hotel, we just welcomed them to our house since we can still accommodate them. We have space at least in the living room for them to sleep. Mara, female immigrant-host, Taal family

There were commonly held expectations of New Zealand recounted by several guests with regard to their visit. Considering that they were first-time visitors to New Zealand, which possesses awe inspiring variance in topography, unique cultures and diverse 
opportunities to experience, several guests expressed similar and familiar expectations about their upcoming visit to New Zealand. Instead of providing hard facts, specific activities, or descriptors gathered from accurate accounts of the place provided by their hosts, the guests provided typical examples of commonly held anecdotal expectations of New Zealand:

See some sheep and cows. Clara, female visiting friend of Taal family

That it [New Zealand] would be as beautiful as I imagined it to be. Then, ah, I've heard there are [as] many cows compared to the people. Male visiting friend of Pinatubo family

I want to see as much as I can. I want to maximise my time - and -- what else -- I just want to look around. I'm not into shopping but I just want to see the scenic spots of New Zealand. Female visiting friend of Ragang family

Well, I expected to check around New Zealand, because I'm into sightseeing and New Zealand is a famous place, like for example, Milford Sound. I research about the Milford Sound, Arthur's Pass -- so of course I am focusing on the conference but like in all other conference, I usually tour around the place. Male visiting friend of Halcon family

However, the expectations for the trip were less descriptive for the Mayon family's friend as the guest expressed being comfortable and relaxed with regard to the preparations of her host, who is a long-time resident to New Zealand:

I am not expecting anything because I know that they have planned everything for me. They plan for what they want to show me. It just happened. Female visiting friend of Mayon family

In the context of friendship, one expects friends to provide support and are surprised if they let the person down (Annis, 1987). Unlike the anticipation and planning made by the immigrant-hosts, the guests were more tied towards logistical preparations such as applying for a visitor visa and their other motivations of visiting New Zealand while the role of the immigrant-hosts was tied to providing advice about travelling to New Zealand (i.e., what to bring or the customs clearing process). This also reflects that anticipation and planning was more of an activity for the immigrant-hosts given that Filipino guests expressed an expectation that immigrant-hosts provide first-hand information about the place. From the perspective of the immigrant-host families, the expectation to host is also connected to their anticipation and planning for their 
guest's $\left(s^{\prime}\right)$ visit and was more focused on looking after the enjoyment and wellbeing of their guest(s):

We just found out that she's coming since we were also invited in the wedding where she [visiting friend] is attending. The bride did not mention that Manang $^{8} \ldots$ will be there although we will really attend since we were invited as early as January so we have prepared for our leave. When I later on found out that Manang is coming, I have maximised my leave. Immigrant-host mother, Mayon family

Our preparation is to accommodate her and ensure that everything is provided. We actually spend everything for her visit and her tour here to see the views and the tourist attractions. Immigrant-host father, Mayon family

Nothing really maybe except that I hope he would somehow enjoy his stay here. Immigrant-host wife, Halcon family

I expected that she will enjoy her tour and that her stay will be memorable. Immigrant-host wife, Ragang family

For the Pinatubo family, as Maria's partner and the guest had not yet met one another, the act of hospitality that is expected of Leonora (being a secondary host in the family) is "getting along" with the guest (or pakikisama) while also allowing the time and opportunity for Maria to socialise with her friend in their residence whenever possible:

I don't know him [the guest] that much, but I tried to be as hospitable as I could be. That's all. However, I think one of my expectations was that I would have to give them space to catch up. Leonora, female immigrant-host, Pinatubo family, individual interview

Regardless of whether the visitor was an invited or an un-invited guest, the unwritten contracts of friendship are focused on expectations of aid and/or solicitous behaviour growing out of assumed bonds of investment, commitment, and reward dependability which fulfil a friend's need (Wiseman, 1986). This is apparent among the immigrant-hosts who are expected to carry out their duties as hosts for their friends. After providing the context of the visit in this section, examining VFR travel using Backer's (2012a) definitional model (in Figure 2.1,

\footnotetext{
${ }^{8}$ An llocano term which refers to older sister; it is a term of respect but not comparable to the English word "Ma'am.
} 
Chapter 2) may too narrowly define what constitutes VFR travel in the context of the hosts and VFs interactions by simply basing the context on the purpose of the visit and type of accommodation without really examining the exchanges (regardless if they were mutual or not) and what the meanings of friendships are for the hosts and guests. For instance, while the guests of the Halcon, Mayon, and Pinatubo families have other motivations for visiting New Zealand, immigrant families choose to take their visits to the country as an opportunity to host for their respective friends, to see them again and to re-establish their bonds of friendship. Once VFs establish their plans for an overseas trip, the chance to reconnect with their friends are made and the VF's travel are viewed as an occasion to expand upon the trip's main purpose, particularly by the immigrant-hosts who want to make their friend's trip worthwhile by escorting them to other places and there is also a desire to see them and to please them. The same scenario occurs for the Ragang and Taal family's guests who have other purposes for visiting New Zealand but they asked their host to act as "broker" and facilitate other aspects of the trip. A descriptive overview of the multi-destination trips of the VFs is presented in the next section.

\subsection{Multi-destination trips of VFs}

The trips and visits undertaken by VFs are frequently a product of complex interactions with a number of hosts. When the VF's visit occurs with more than one host, they carry out a series of visits with either VFs or VRs in a range of places. One factor that influences the multi-destination trips of VFs as itinerant guests is collaborative hosting responsibilities shared between and/or within the respective immigrant-host families. There are two different types of collaborative hosting: (a) within the household and (b) outside the household. An example of collaborative hosting outside the household includes contacting relatives and/or friends elsewhere in New Zealand (usually Filipinos) to accommodate their guest during other phases of the trip. With regard to collaborative hosting within the household, the obligation to serve the guest(s) is something that is done in cooperation with the rest of the members of the immigranthost family. Currently, there are insufficient studies on the role that residents play in 
stimulating and influencing tourism activities for their visitors (McKercher, 1996; Young et al., 2007) and the dynamics occurring particularly at the household level. Even outside a particular household, relatives of immigrant-host families are also frequently engaged and considered in planning the trip of their guest.

As interview transcripts were coded and analysed in the process of examining the patterns of social interaction between the immigrant-hosts and VFs, tables were used to analyse and represent the complexity of trips or activities undertaken by the guests and how the hosts are able to influence their trips in New Zealand (see Tables 4.2 to 4.6). These were useful to map out the activities undertaken by the immigrant-hosts and the guests and in examining the geographical pattern of the visit or hosting (e.g., Lew \& McKercher, 2006; Lockyer \& Ryan, 2007). The destinations in New Zealand were highlighted using a red font to show the places visited by VFs.

However, social relationships in the context of VFR travel involving immigrant-hosts and guests are complex. Coles, Duval, and Hall (2004) and Hall (2005) suggest that the conceptualisation and development of theoretical approaches to tourism should consider relationships which include VFR travel. In this case, social interactions become nested relationships between the immigrant-hosts and guests that may involve multiple actors within a network in varying degrees. Hence, each case is examined by way of presenting them on a table where the relationships between the immigranthosts and their VFs are outlined while including other individuals who may have been involved in the social interactions or those who may not have been interviewed but may have "indirectly" influenced the social interactions between immigrant-hosts and VFs and hosts/guests who were not interviewed due to their inaccessibility (as explained previously in the methodology section). However, a deeper understanding and contextualisation of social exchanges and emotional solidarity between actors are further highlighted in sections 4.4 and 4.5 as they deal with the past interactions between hosts and guests and their solidarity which is largely the product of their social interactions. 
Group dynamics within immigrant-host families and their respective visiting friends were difficult to examine as the relationship varies between them and in the involvement of other members in the family. The relationship between a host and visiting friend(s) were initially thought to be dyadic, which means that the bond (or exchanges) only exists between one specific host and one specific guest. However, in the case of the Halcon, Pinatubo and Taal families (see Tables 4.2 to 4.4), other members may be involved in the social interactions. For instance, the immigrant-host husband in the Halcon family and the male visiting friends are childhood friends (Table 4.2). Obviously, the bond between the immigrant-host wife and the visitor is not as apparent when compared to that of her husband, which includes both historical and social ties with their guest. However, the gendered role of the wife as a secondary host within the family is revealed further in the latter section (section 4.6) to show how she was also involved with and influenced by the guest's visit. While the male visiting friend was hosted by the Halcon family in Wellington, he was also accommodated by the sister of the immigrant-host father in Auckland, even though the primary purpose of the guest's visit in Auckland was to attend a conference.

Table 4.2. Social interactions between Halcon family and visiting friend.

\begin{tabular}{|c|c|c|}
\hline Host(s) & Guest(s) & Key Characteristics of the Visit \\
\hline \multirow[t]{4}{*}{$\begin{array}{l}\text { Host-husband } \\
\text { Host-wife }\end{array}$} & \multirow[t]{4}{*}{$\begin{array}{l}\text { Male visiting friend } \\
\text { of the host- } \\
\text { husband }\end{array}$} & $\begin{array}{l}\text { Attending a conference, the male guest was } \\
\text { also accommodated by host-husband's sister } \\
\text { in Auckland }\end{array}$ \\
\hline & & $\begin{array}{l}\text { Male guest attended a conference in } \\
\text { Auckland }\end{array}$ \\
\hline & & $\begin{array}{l}\text { Male guest was accommodated by Halcon } \\
\text { family in Wellington }\end{array}$ \\
\hline & & $\begin{array}{l}\text { Male guest was accommodated in } \\
\text { Christchurch by his girlfriend's relatives } \\
\text { together with his girlfriend. }\end{array}$ \\
\hline
\end{tabular}

Note: Two toddlers of the host family (not interviewed); male friend was a lone visitor.

In the case of the Pinatubo family, only Maria (within the Pinatubo family) and the male guest were close friends (Table 4.3). Nonetheless, Leonora being the secondary host within the household assisted in hosting the guest which included installing a bedframe in the guestroom and taking their visitor around Wellington despite the fact that the 
visitor was her partner's friend. The selection of activities for the male visiting friend was arranged by both hosts and decided mutually within the host family unit.

Table 4.3. Social interactions between Pinatubo family and visiting friend.

\begin{tabular}{|l|l|l|}
\hline \multicolumn{1}{|c|}{ Host(s) } & \multicolumn{1}{|c|}{ Guest(s) } & \multicolumn{1}{c|}{ Key Characteristics of the Visit } \\
\hline Female host (Maria) & $\begin{array}{l}\text { Male, visiting friend } \\
\text { of Maria }\end{array}$ & $\begin{array}{l}\text { Male visiting friend was accommodated by his } \\
\text { niece in Auckland }\end{array}$ \\
\cline { 3 - 3 } $\begin{array}{l}\text { Female host } \\
\text { (Leonora) }\end{array}$ & $\begin{array}{l}\text { From Auckland, Pinatubo family paid for the } \\
\text { trip to go to Wellington (return) }\end{array}$ \\
\hline
\end{tabular}

Note: Host family consists of lesbian couple; male visiting friend travelled to New Zealand for a holiday.

In the Taal family, the inter-relationship between Mara and the guests is derived out of the need to conform to her former partner's wishes, and Mara's relationship with Clara would only function due to her sense of obligation to accept guests within the household as she and her ex-partner were cohabiting at the time of hosting (Table 4.4). This relationship may also be viewed from the perspective of the deeper sense of relationship that typically exists between relatives; that is to say, between Mara's former partner and his visiting relatives (auntie, uncle, and cousins). Mara remains very civil and cordial as she accompanies her former partner and his guests for dinner and even while traveling outside of Auckland. Clara and her family also visited other friends on this trip:

They actually didn't go out much because they didn't like the weather here. They find it very cold. So, instead of them going out they just stayed inside. We just go out when we have dinner or during the weekend before they left. We went to Rotorua and then a long drive to Hobbiton in Matamata. They shared with the expenses. Mara, female immigrant-host, Taal family

[We also visited other places]. They are good family friends. They are all staying in New Zealand, like three families, like three siblings so all the [family name] are staying there so we visited them. It was like a big reunion to everyone. The [family name] were our neighbours before and [name of cousin] live in the same area. They all have been staying in New Zealand for like ten years. So, we just visited them. Clara, female visiting friend of Taal family 
Table 4.4. Social interactions between Taal Family and visiting friend(s).

\begin{tabular}{|c|c|c|}
\hline Host(s) & Guest(s) & Key Characteristics of the Visit \\
\hline $\begin{array}{l}\text { Female host (Mara } \\
\text { as secondary host) }\end{array}$ & \multirow[t]{2}{*}{$\begin{array}{l}\text { Female visiting } \\
\text { friend of Mara } \\
\text { (Clara) }\end{array}$} & $\begin{array}{l}\text { The guests were accommodated in the } \\
\text { residence of Mara and her former partner in } \\
\text { Auckland }\end{array}$ \\
\hline \multirow{2}{*}{$\begin{array}{l}\text { Ex-partner of } \\
\text { Mara/a cousin of } \\
\text { Clara } \\
\text { (not interviewed) }\end{array}$} & & $\begin{array}{l}\text { The group of guests were assisted by the ex- } \\
\text { couple in Rotorua and Hamilton (Hobitton) }\end{array}$ \\
\hline & $\begin{array}{l}\text { Three other } \\
\text { relatives of Clara } \\
\text { including her } \\
\text { parents and brother } \\
\text { (not interviewed) }\end{array}$ & $\begin{array}{l}\text { The guests were assisted by the hosts to } \\
\text { meet other family friends in Auckland }\end{array}$ \\
\hline
\end{tabular}

Note: Due to personal circumstances, the former partner of Mara and the other members of the visiting group were unavailable and were not interviewed in this study. Mara knows the relatives of her former partner from her previous residency in the Philippines.

The Ragang family's social interactions with their guests were more complex (Table 4.5) as several parties were either "indirectly" involved, but the principal host and principal guest uniting the parties are the immigrant-host mother and her visiting best friend. On the host side, the immigrant-host mother has arranged for the trip of the visiting group to Auckland, but also to Rotorua and Taupo with her brother acting as the visiting group's guide or secondary host, who was their driver as the immigrant-host husband could not join them due to his work obligations. The guests of the Ragang family were also invited for a dinner given by the relative of the host in Taupo. On the guest side, the best friend deals with the Ragang family on behalf of the visiting group while the mother of the female visiting friend has another purpose for visiting New Zealand. Also, while visitors of the Ragang family were escorted around the North Island, they arranged for a separate package tour in Queenstown which did not include an escort from the immigrant-host mother of the Ragang family. 
Table 4.5. Social interactions between Ragang Family and visiting friends.

\begin{tabular}{|c|c|c|}
\hline Host(s) & Guest(s) & Key Characteristics of the Visit \\
\hline \multirow{2}{*}{$\begin{array}{l}\text { Host-wife } \\
\text { Brother of host- } \\
\text { wife (as driver; not } \\
\text { interviewed) }\end{array}$} & $\begin{array}{l}\text { Female visiting } \\
\text { friend (best friend of } \\
\text { host-wife) }\end{array}$ & $\begin{array}{l}\text { The entire visiting group was accommodated } \\
\text { by the female visiting friend's uncle in } \\
\text { Auckland }\end{array}$ \\
\hline & $\begin{array}{l}\text { Mother of female } \\
\text { visiting friend }\end{array}$ & $\begin{array}{l}\text { Guests were escorted by host-wife (together } \\
\text { with her brother as the driver) within } \\
\text { Auckland city and out-of-town trips in } \\
\text { Rotorua and Taupo }\end{array}$ \\
\hline \multirow{3}{*}{$\begin{array}{l}\text { Relatives of host- } \\
\text { wife in Taupo (not } \\
\text { interviewed) }\end{array}$} & \multirow{3}{*}{$\begin{array}{l}\text { Three other } \\
\text { friends/relatives of } \\
\text { female visiting } \\
\text { friend (not } \\
\text { interviewed) }\end{array}$} & $\begin{array}{l}\text { Guests used commercial accommodation in } \\
\text { Taupo; treated for dinner by host-wife's } \\
\text { relatives in the said area }\end{array}$ \\
\hline & & $\begin{array}{l}\text { Guests used commercial accommodation in } \\
\text { Queenstown but were unescorted by Ragang } \\
\text { family }\end{array}$ \\
\hline & & $\begin{array}{l}\text { Guests were treated for a dinner by host- } \\
\text { wife's husband in Auckland }\end{array}$ \\
\hline
\end{tabular}

Note: The Ragang family is composed of host-wife with her other relatives as 'indirect' hosts for their guests composed of her best friend and four other companions.

The Mayon family and their female visiting friend represent multilateral social interactions as the entire immigrant family group was directly involved in hosting for their guest. Social exchanges occur and a common solidarity is felt across all members of the interacting group. As for the immigrant-host family, while they had the opportunity to travel together with their friend in the North Island. They also arranged a different independent trip for their guest to maximise their friend's trip to New Zealand, which was paid for by the host family. The immigrant-host family encouraged and arranged for their guest to travel to the South Island even if she is unescorted by them:

We looked for someone to host her. One good thing is that, my cousin who worked before in South Island for one and a half years is now living in Wellington, and they looked for other Filipinos to accommodate our visiting friend. Then, I just paid for her accommodations in South Island to make sure that she is toured around. Immigrant-host mother, Mayon family, individual interview

Through her own expense [to travel to New Zealand]. [However,] We guaranteed her that once she arrives, we would shoulder all the expenses here. So, she did not spend anything when she came here. Even when she went to Queenstown by herself, we arranged for her accommodation and her flight going to Queenstown to stay with another friend over there. Immigrant-host father, Mayon family, individual interview 
Table 4.6. Social interactions between Mayon family and visiting friend.

\begin{tabular}{|c|c|c|}
\hline Host(s) & Guest(s) & Key Characteristics of the Visit \\
\hline \multirow[t]{6}{*}{$\begin{array}{l}\text { Host-father } \\
\text { Host-mother }\end{array}$} & \multirow{6}{*}{$\begin{array}{l}\text { Female visiting } \\
\text { friend of the host- } \\
\text { father and host- } \\
\text { mother }\end{array}$} & $\begin{array}{l}\text { The female visiting friend attended a friend's } \\
\text { wedding in Auckland and was seen by the } \\
\text { Mayon family during the celebration }\end{array}$ \\
\hline & & $\begin{array}{l}\text { From Auckland, the host parents for the } \\
\text { Mayon family drove together with their guest } \\
\text { to Hamilton to stay with the host-mother's } \\
\text { brother }\end{array}$ \\
\hline & & $\begin{array}{l}\text { Drove to Rotorua and stayed in commercial } \\
\text { accommodation }\end{array}$ \\
\hline & & $\begin{array}{l}\text { From Rotorua, went to the host's residence in } \\
\text { Wellington and stayed for a couple of days }\end{array}$ \\
\hline & & $\begin{array}{l}\text { From Wellington, the host family arranged a } \\
\text { trip for their guest in Queenstown by looking } \\
\text { for other Filipino friends in the said area. } \\
\text { Though unescorted, the Mayon family } \\
\text { coordinated and paid for the guest's trip }\end{array}$ \\
\hline & & $\begin{array}{l}\text { Returned to Wellington after the trip and } \\
\text { went back to Auckland to stay with another } \\
\text { friend }\end{array}$ \\
\hline
\end{tabular}

Note: One son of the immigrant-host family who is a minor was not interviewed.

Travelling allows individuals to turn to others (including friends) within their social network for emotional support as well as help in decision-making as they represent relatively low-cost heuristic solutions (Ryley \& Zanni, 2013). Immigrant-hosts may, on occasion, provide logistical support, accommodation, and may also link their guests to other friends or relatives in their new homeland in order to facilitate other side trips to maximise the visit of their VFs. This also underpins the fact that VFs undertake several side trips within New Zealand which can be a complex process and would take into account preferences, constraints, and the extent of personal networks. As the male visitor of the Pinatubo family stated:

It made it more enjoyable and, easier in terms of, you know, being with them and staying with them. You know that they are Filipinos and they would understand your needs and requirements as a visitor. It becomes spontaneous that they would take care of your accommodation, food or bringing you around - and then the expenses that go with the tour. 
While there are occasions for immigrant-hosts and VFs to be together, they also have other social networks in New Zealand which they are also maintaining and this motivates them to travel to other places. Particularly, VFs are being hosted or accommodated by other host families in New Zealand (usually other Filipinos) which would mean they are itinerant guests, yet, they may be unescorted by their immigranthosts from place to place. The types of activities undertaken by VFs during their visits are influenced not only by one host as they negotiate a series of activities using one's social network but also by other secondary hosts who may live in different places in New Zealand.

As friendships are developed and nurtured over time, such relationships are maintained by first-generation migrants with their guests and are rooted in their past interactions in the Philippines $^{9}$, which are contextualised in order to deeply comprehend and interpret the meanings of their social interactions (discussed in detail in section 4.7). Furthermore, social exchange theory and the theory of emotional solidarity are being utilised to help to better understand the social interactions between immigrant-hosts and VFs as phenomena.

In brief, the multi-destination trips that are undertaken by guests frequently have several motivators at different destinations that may or may not be influenced by and/or affect one or more of the host families, making the VFs itinerant guests (discussed in section 4.2). Regardless of the motivation(s) for the guest(s) to travel overseas or for being invited, hosting for friends on the part of the immigrant involves caring for and being concerned about the welfare of the visiting friend. Friendship is an example of a relationship that sometimes produces special duties or obligations, such as witnessing a celebration with a friend or experiencing a mutually rewarding event or destination. Likewise, hosting a friend for a visit is another of life's special duties that one does occasionally and is a specific duty that one does for a friend which is not usually extended to individuals beyond family members. In friendship, there is a mutuality of affection, sharing, concern and trust. This mutuality is the basis of special

\footnotetext{
${ }^{9}$ Or elsewhere, for the case of Maria in Pinatubo family.
} 
responsibilities (Annis, 1987) and is a characteristic of friendship and is an essential element in a social exchange which is further elaborated on the next section.

\subsection{Past interactions and social exchange}

In order to have a deeper understanding of the social exchanges between immigranthosts and VFs, there is a need to examine their past interactions so as to provide a perspective of what constitutes the contours and gradations of their relationship. Friendships may involve social exchanges that are developed over time resulting in a special bond that produces certain responsibilities which are understood or assumed. Providing a context of the past interactions between immigrant-hosts and VFs would be useful in order to understand the mutual exchanges that produced their social interactions and in terms of how their emotional solidarity is felt and experienced (see section 4.5).

Social exchange theory is the underpinning principle that guides in addressing the second supplementary question: "What are the social exchanges between the immigrant-hosts and VFs?" It is through VFR travel that social exchanges between immigrant-hosts and their visiting friends, who may no longer reside within a reasonable proximity of each other, can continue face-to-face interactions giving them the opportunity to reminisce about their past social interactions, share personal stories, and strengthen and maintain their future friendship ties. As a social exchange, the obligation of hosting or visiting in and of itself means that there is an on-going relationship that the participants want to maintain, even if it is across great distances.

Social exchanges also depend on the degree of depth of friendship ties, which may not necessarily be mutually or uniformly felt across a specific group of hosts or guests and will also depend on the motivation of a specific party. Friendships are built over time and the past behaviour, understandings, expectations, and loyalties create a special bond that produces special responsibilities (Annis, 1987) including hosting and visiting friends. Friendship and social exchanges between immigrant-hosts and VFs are rooted 
in their past interactions, and particularly in the Philippines where they had established and strengthened their ties until the hosts eventually migrated to their new homeland. As explained earlier in Chapter 3, this research recognises that there are other types of friendships that may be formed or sustained which was not a characteristic of the research participants involved in the study, such as those friendships formed across great distance (e.g., pen pals), virtually (e.g., dating sites, social media), or on-line romantic relationships. However, the friendships that were formed by the research participants were all created through personal face-to-face interactions prior to the hosts migrating to New Zealand.

One of the basic tenets of social exchange is that relationships evolve over time into trusting, loyal, and mutual commitments (Cropanzano \& Mitchell, 2005) which may also weaken and diminish. As such, the reciprocal forms of exchange (of hosting/of visiting) provide benefits to each other without each participant actually knowing what returns, if any, that they will receive. As a social exchange, the obligation of hosting or visiting in and of itself means that there are on-going relationships that the participants want to maintain even across great distances. To further facilitate the analysis of the social interaction between the immigrant-host family and the VF and understand the social exchanges between friends, their past interactions are individually enumerated to provide the context of their relationship which began in the Philippines, such as:

She is our friend and ninang (godmother) in our wedding, a primary sponsor in our wedding. We've known her for so long. She was an officemate of my wife's in __ [...] We have known her (visiting friend) for around 40 years, even before my wife and I got married. Immigrant-host father, Mayon family, individual interview

In the Philippines, the social system of ninong and ninang (the male and female sponsors, respectively) at weddings involve an obligation to provide life-long counsel and wisdom to the couple being married. In a typical Filipino wedding, one can count several ninongs and ninangs who stand behind the couple, pledging their support and the promise of guidance and care (Song, 2006). Even if the Mayon family migrated to New Zealand a long time ago, social exchange still occurs and is manifested in hosting for their ninang. These are friendships that are reinforced and the ninang is expected 
to serve the role of the couple's "second parent" or "counsellor" especially when the newlywed's parents are deceased and are no longer able to serve as their guide. This cultural (and spiritual) practice in Filipino celebrations, including weddings, reinforces the friendship bond which binds the Mayon family and their guest. Within the context of Filipino culture, godparents are examples of an invitation to become an extension of a family (Gough, 2001). Therefore, a hybrid friend/family relationship or an overlap between family and friends occurs.

A similar bond exists between the immigrant-host father of the Halcon family and their respective male VF as the latter was a godfather to one of the Halcon family's children in a baptismal celebration. The traditional concept of god parenting makes a co-parent equally responsible for ensuring the child's healthy upbringing and spirituality. This bond also designates that this individual should be the child's guardian should he or she become orphaned. Being a godparent is therefore being "a presence" in a child's life but requires a moral responsibility and could entail a lifelong parental commitment. The establishment of such relationships among equals is generally a symbol of mutual trust and confidence and a commitment of each to assist one another. However, past interactions still stem from being long-time friends:

We're of the same age. When I first saw him, I threw a stone at him. I was curious because my family were long-time residents in the province. Probably because he is the only child, his mother invited me to watch a movie. [...] And then every time there is an occasion, say, a birthday, we are invited. And since he is the only child, he always comes to our home and see my parents as well as my other siblings. Then, we went to the same primary school. During high school, I went to a different school but in university, we were still together. He even stayed in our place. Oh no, I mean, that was when he went to law school. Immigrant-host father, Halcon family, individual interview

The following statement made by the immigrant-host father resulted when the researcher asked about what constitutes their friendship and how each of their families is related to one another:

Yes, [he is] a family friend. My father has even joked with him, "You should start changing your last name." [laughs]. He attends different functions within my family such as my parents' wedding anniversary. As well, his girlfriend is a former classmate in high school and her parents are based in 
Christchurch. I think nothing has really change -- more or less; we are at ease with one another. Immigrant-host father, Halcon family, individual interview

In contrast to the preceding account of a lifelong multidimensional relationship, Maria's (Pinatubo family) uncomplicated friendship with her guest developed at a later stage in life, during her professional career, as she and her guest met when they were overseas scholars:

Well, he is a very good friend of mine and so very close to me when we were in Malaysia. We haven't seen each other for perhaps seven to ten years. I don't know - but the last time we saw each other was when we were in Japan. So, after Malaysia, we went to Japan and we went to different universities so we parted ways. We went to Japan [in] 2003 and probably he visited me at my place in 2004. He stayed with me for a couple of days for a visit and after that we haven't seen each other since. Maria, female immigrant-host, Pinatubo family

Meanwhile, the immigrant-host mother for the Ragang family and her visiting friend have a long and very close relationship as they were classmates in the Philippines and have known each other for over two decades. During the interview, a probing question to clarify what the relationship means to her when she said, "best friend":

She's my close friend - as in a confidante in college. We shared so many common things. Immigrant-host mother, Ragang family

The Ragang immigrant-host mother's VF also recounted a similar interpretation of their relationship:

Female visiting friend: We became friends because we were classmates -that was how many years back when we were just in college [university].

Interviewer: So that was like, ten years? Twenty years?

Female visiting friend: Yes, that was in 1980s -- late 80 s. We were classmates. We studied together. During our spare time, we are always together. So, that's how our friendship started and it has grown over the years. 
The period when the immigrant-host mother in the Ragang family and her guest became best friends was during adolescence, a period when teenagers (including university students) explore their sense of identity as they search for a group to which they relate or belong. Therefore, this can be an intense and frequently emotional period of development where mutual exchanges and trust are established between Filipino adolescents. This also suggests that social exchange within interpersonal relationships exist as a pattern of exchanges of material and perhaps non-material rewards (e.g., psychological) leading to commitment in keeping their emotional investment and the relationships intact.

Of the five immigrant-host families who have hosted for their friend, Mara's case was different as she was cohabiting with her ex-boyfriend at the time of her hosting. Although she casually knew her visitors while she was still residing in the Philippines, there was a need for her to conform and to be accepting as her former partner and housemate hosted for his relatives:

She is the cousin of my former partner. They travelled together as a whole family - the dad, mom, and two children, four of them. They are my former partner's relatives. Since we are living in the same house, my relationship to them is friends. Mara, female immigrant-host, Taal family

Social exchanges are also manifested in the side trips and visits taken by VFs in the company of their immigrant-hosts. As friendship is a multidimensional experience, the shared or communal nature of friendship allows individuals to participate in activities of mutual interest (de Vries, 1996) whether through hosting or visiting. By way of social exchange, the occasion of a visit gives an opportunity for friends to exchange stories, reminisce about their past interactions, and get re-acquainted after they have been separated since the immigrant-hosts left their former homeland (the Philippines). The relationship has continued as they still have mutual trust in each other which allows the sharing of personal intimacy. Liking implies enjoying the person and being concerned about the person which gives rise to seeking out the person's companionship and doing things together (Annis, 1987). Through social interactions, the mutual exchanges provide the immigrant-hosts and VFs the opportunity to become re-acquainted which is recounted in the following quotation from the immigrant-host parents of the Mayon 
family who describe what they felt and experienced during the visit of their friend:

We enjoyed dining together, sharing stories, what we did 40 years ago when we [wife and I] were still single and we're going out together - stories from way back. Of course, you catch up, recall stories and ask what happened on her side when she was away from us and what happened on our side. Immigrant-host father, Mayon family individual interview.

During the wedding, I was happy to see her as if nothing had changed. We hadn't seen each other since 2007 . She still looked very young. I was so excited and we were very happy. We [my family] were looking forward for her visit to Wellington. Immigrant-host mother, Mayon Family, individual interview.

How will I describe it? It's like nothing had changed. If we would see each other again here or in other places, it would be the same. Only our looks or faces will change but our relationship remains. Female visiting friend of Mayon family

Evidently, social exchanges are re-established after periods of separation through the visiting of friends. Social interactions in the form of a visit enable the compressing of time commonly called "catching-up", with the function of rekindling the significant memories that they previously shared and informing each actor of the significant happenings and situations that occurred in the life of the other during their separation:

At first, I was a bit unsure of how I will be reacting to her since the last time we saw each other was 1995. What happened was that when I gave birth, she went home to [name of province]. I only met her once but, since then, we never saw each other again. As time goes by, you don't know whether her ways or attitudes have changed. However, she has not changed. She maintains a low profile, like being thrifty when it comes to spending. Immigrant-host mother, Ragang Family

First of all, I haven't seen her for a long time. I have to renew my ties. I wanted to know anything new about her. What happened to her in between [those times that we parted ways]. You know, rekindling ties with friends. Of course, friends that you value -- because there are also types of friends that it's alright not to see them. Female visiting friend of Ragang family

From the preceding quotations, it is clear that social exchanges are quickly redeveloped over the course of the visit as friends share or exchange stories about past interactions in order to re-acquaint and renew their friendship ties. These on-going relationships are 
once again nurtured as they reminisce about the time they spent together before and considering that they have less regular contact after the immigrant-host may have migrated to New Zealand, the actors agree that the bonds still remain.

Social exchange in the context of friendship is a multidimensional experience where hosting becomes an invitation for the immigrant-hosts to share their private life with friends and allows them to continue their relationships that were forged overseas in the Philippines. Through social exchange, the occasion of a visit gives an opportunity for friends to reconnect after they have been separated since the immigrant-hosts emigrated from their former homeland (the Philippines). The relationship persists as they still have mutual trust in each other which allows the sharing of personal intimacy.

Social exchange may cultivate a more fertile ground for the development of mutual trust and future exchanges whether through hosting/visiting in New Zealand or in return visits of immigrant-hosts to the Philippines. While a specific immigrant-host family member may equally provide (or distribute) resources to a group of visitors, or vice versa, understanding Filipino culture and emotional solidarity which exists between the host's $\left(s^{\prime}\right)$ and guest's(s') social interactions cannot be directly understood by only using social exchange theory. As emotions are central to interactions (Fields, Copp, \& Kleinman, 2006), the theory of emotional solidarity will guide the analysis presented in the next section.

\subsection{Emotional solidarity: connectedness in togetherness}

Friends strive to create time and space for repeated meetings, conversations and joint actions, just as religious believers set aside time for their participation in sacred rituals (Wallace \& Hartley, 1988, p. 97).

The above quotation illustrates the emotional solidarity between and among friends as this section addresses the question: "How do social interactions reflect emotional solidarity between the immigrant-hosts and VFs as expressed by their shared beliefs and behaviours?" As the most frequently identified dimension of friendship is its affective 
nature, the theory of emotional solidarity is used as another concept apart from social exchange theory to understand the social interactions between immigrant-hosts and VFs. Friendship involves both social exchanges and emotional solidarity and this section will focus on friendship from the perspective of emotional solidarity. Significantly, the affective nature of friendship includes the sharing of personal thoughts and feelings (i.e., self-disclosure and self-expression), other related expressions of intimacy, appreciation, and affection (including respect and feelings of warmth, care and love) (de Vries, 1996) which is obviously expressed through hosting and visiting for friends or established through one's co-presence.

Utilising Durkheim's theory of emotional solidarity (Durkheim, 1915/1995; Woosnam \& Norman, 2009; Woosnam et al., 2009) in the context of the host-guest social interaction, all parties interact and assume that they still share common beliefs and behaviours which eventually fosters emotional solidarity. Emotional solidarity becomes an affective bond that individuals experience with one another and is characterized by perceived emotional closeness and degree of contact (Hammarstrom, 2005; cited in Woosnam \& Norman, 2009). Such emotional solidarity is made apparent through the co-presence of friends - regardless of their role, be it host or guest.

Social interactions in New Zealand, as a home for immigrant families and as a place visited by their guests, are both actively produced and nurtured through the togetherness of the hosts and guests. Therefore, through VFR travel, New Zealand becomes a place for the participants' face-to-face social interactions, about continuing their relationships, and about the placing of peoples in relation to their being hosts/guests and as friends. Immigrant-host families with whom they intimately share their friendships with their guests (and vice versa) perceived their togetherness or copresence as memorable. Being together may also be influenced by the places and/or activities shared by the social interactions between immigrant-hosts and VFs. Some immigrant-hosts recalled that their hosting activities were very much enjoyed by their guests which bolstered their concept of New Zealand and of being New Zealand residents as hosting can enhance their sense of home (Griffin, 2013) and particularly so 
for new immigrants who only had the opportunity to explore places in their new homeland when they hosted a guest.

Both hosting and visiting are necessary to social life where corporeal co-presence is essential in fulfilling social obligations (Larsen et al., 2007). Analogously, hosting and visiting are all important to having a vibrant social life, remembering their past interactions together in New Zealand or other places and nurturing their friendships:

When we were seated in a bench in Lake Taupo and the background looks very beautiful. We had our photo taken together and thought we should print a bigger version. It reminds us of the good old days and a chance to catch up about what we have missed. Immigrant-host mother, Ragang family

What is memorable is the bonding that we share and the time together and exploring different places. Even the host hasn't gone to those places. It's something new to me and to her also. Female visiting friend of Ragang family

Hosting and visiting in the context of VF travel is a way of acting and being with friends in real time which affords the opportunity for shared experiences which facilitates advancement of the relationship. Hosts and guests feel valued and cared for through the generosity and reciprocal nature of the interactions that occur during a visit. Therefore, they feel that the relationship benefits them and as a result is made more significant. Likewise, the host who has felt emotional solidarity from the co-presence of a VF expresses strong sentiments of appreciation for the opportunity of the visit. The visit has the effect of a lens in that it magnifies the significance of events for the players and they agree events seem to be more meaningful when shared with a friend:

When we rented a unit in Rotorua which is like a house, we reminisce those times when we go out of town in Pangasinan. ${ }^{10}$ We cook, buy groceries. We don't eat outside. We cook food while sharing stories. Then, when you wake up, you are still together with friends. That was wonderful. Immigrant-host mother, Mayon family, individual interview

When I am together with their family - eating together or simply those chat we have. Female visiting friend of Mayon family

${ }^{10}$ The province of Pangasinan is located in the north-western part of the Philippines where scenic spots like the Hundred Islands is located. 
From the reading of the above quotations, emotional solidarity varies among the immigrant-hosts and guests. For those immigrant-hosts and VFs who travel together, the places being visited and the situations experienced in a place can create emotion and the place can become as significant as the regard that they hold for an individual. For the immigrant-hosts, this effect may enhance their appreciation of New Zealand as a place. However, another significant aspect of emotional solidarity is that even when they are at home and performing life's routine duties, the immigrant-hosts and VFs social interactions transform the "ordinary" experience into something "extraordinary" through their co-presence.

VFs who may not share an intimate relationship or strong bond with their immigranthost(s) and do not perceive togetherness with their immigrant-host(s) as a factor that influenced their decision to visit to New Zealand. This is reflected by the relationship between Mara and Clara where Mara feels compelled to accommodate Clara as she is a cousin of her former partner with whom she is still cohabitating. In this case, the VF (Clara) does not associate any sentiments of co-presence with the host or in relation to her experience in New Zealand:

Interviewer: What was the most memorable aspect of your visit to New Zealand?

Clara: I think the sheep. I like the sheep. Feeding the sheep and playing with them since we don't have them here. It's just like we don't have a snow in the Philippines.

Interviewer: That's something memorable for you?

Clara: Yes. Then, there are no wild animals that could kill you in New Zealand. Generally, the people are safe from animals.

While the host-wife of the Ragang family and her VF are the best of friends, in contrast the VF's mother expressed a different impression of her visiting experience as she had a different motivation to visit New Zealand. Also, the visiting mother's relationship with the host-wife (for the Ragang family) is 
evidently not as deeply rooted as her daughter's friendship. So, despite being hosted and toured in the same caring fashion by the same family as her daughter, the VF's accompanying mother could not provide meaningful and specific details of her interactions with her hosts:

Mother of female visiting friend: Memorable? Everything was memorable.

Interviewer: If you have to choose the number one on your list, what comes into your mind right away?

Mother of female visiting friend: My brother, we saw each other.

In this case, the visiting mother's trip was meaningful due to seeing her sibling who accommodated them in Auckland. Nevertheless, she still felt in solidarity with her daughter with regard to the impact of the visit and the significance of relationship with their hosts (the Ragang family) on her daughter:

Happy, especially for [name of female visiting daughter]. It has been many years [since they have seen one another].

The methodological approach in examining emotional solidarity across time and space between the immigrant-hosts and VFs social interaction also reveals noteworthy insights particularly for the pre- and post-visit phases. During these phases, there is a tendency for the frequency of communication between immigrant-hosts and their VFs to become occasional or scant. However, the motivation for a friend to resume more regular contact is usually catalysed by the opportunity of that friend to visit New Zealand, thereby causing the potential visitor to initiate contact with their potential immigrant-host friend(s) to determine if there is an interest or ability to host them (earlier outlined in section 4.1 and in relation to anticipation and planning).

Since friendships are uniquely voluntary and a relatively uninstitutionalised relationship (Adams \& Blieszner, 1994; Dreher, 2012; Kenrick et al., 2010; Wiseman, 1986), there is a tendency for the interactions to eventually diminish at the post-visit phase to the standard frequency of interaction that the participants maintained prior to the VF initiating the request to visit (pre-visit phase). It appears that while long distance friendships are highly valued for nostalgic reasons, the immigrant-hosts and VFs in the 
post-visit phase return to their normal routines:

When they got home, ah I called them to check if they got home safely. That's it. Yeah, no more roles after that. Mara, female immigrant-host, Taal family

Yes, it's [communication] always through emails. I always tell him that, "You know it may take long before I respond but, I will always respond." Maria, female immigrant-host- Pinatubo family

Nothing, I guess because my connection with the guest is through Maria, but she is the type of person who is not really active in dealing with friends. She is quiet but the affection remains that our friend are still important. I mean, a friend is important but it doesn't mean you have to talk every day or constantly catch up. Leonora, female immigrant-host, Pinatubo family

We would communicate through e-mail - although not that often - maybe when I see her [Maria] online on Facebook. Male visiting friend of Pinatubo family

Finding time, considering that many have busy lives, to regularly interact with friends and family may be difficult for immigrant-hosts after the visit in New Zealand:

We communicate with her every once or twice a week. Immigrant-host mother, Mayon family

[I don't communicate with her], it's mostly Mom and Dad. Immigrant-host son, Mayon family

Yes, through internet....but not that often as I know they're working and they have a different time zone. I am also busy since I came back. Female visiting friend of Mayon family

Likewise, time spent in maintaining friendships over a distance becomes irregular as both actors may have other priorities during the post-visit phase:

It has somehow diminished because of my work. I did have a lot of concerns like, I still don't have my permanent residency. I was only holding a work permit then. So, that was my limitation. It was a big project to for us to get our permanent residency and get a bigger house for our kids to settle and get a house and a car. Those were my concerns after the visit. Immigranthost father, Halcon family, individual interview

Nothing really [since I don't have any obligations]. Male visiting friend of Halcon family 
This tendency for communications to wax and wane was a common pattern recounted within the individual and group interviews with the hosts and their respective guest(s). The social interactions between immigrant-hosts and VFs indicate that obligations and expectations in the pre- and post-visit phases are less and that mutual aid between friends is based on the need and desire to host/visit, mutual affect, and reciprocating favours. Nonetheless, most VFs have promised to reciprocate the hospitality offered by their respective hosts in the future. While these guests did not explicitly mention the Filipino value of utang na loob (debt of goodwill) which is not governed by any written or formal rules, they put a premium on the goodwill that was conveyed by their hosts, which was made evident as guests expressed the willingness and desire to return the favour and host should the immigrant-host family make a visit to the Philippines. In this context, what the Filipino term utang na loob would actually mean for the VF is reciprocating the hosting for this act was a symbolical gesture of giving part of oneself. In essence, such social exchanges within the Filipino culture means that reciprocating such a gesture should be done out of free will where the former recipient (in this case, the VF) under such circumstances: (a) will not act under external compulsion; (b) is motivated by positive feelings; and (c) is not motivated by the anticipation of reward (see De Castro, 2001).

Despite the vast distance from their former homeland, first-generation Filipino immigrants in New Zealand and elsewhere maintain their relationships with their guests, which were forged through their past interactions in the Philippines (shown in section 4.4: Past interactions and social exchanges) where most friendships had been established a long time before. Filipino immigrant-hosts in New Zealand maintained contact with their VFs in what could be described at best as infrequent or sporadic communication, during their residency periods which were two to four years in length on average. Immigrant-hosts are still maintaining their ties with their friends in the Philippines by inviting or accepting requests to host their guests. However, as distances may separate people, friendships may be continuous, but the communication between and among friends is not. 
The during-visit phase shows that despite limited contact in the pre- and post-visit phases, the social interactions between immigrant-hosts and VFs are sustained through their mutual social relationships that are not based on proximity, but are continued over periods of time between the particular players in the social dynamic. Friendships are therefore being sustained beyond single or short-term encounters which involve the search for some form of sentiment or at least empathy and common ground between persons (Bell \& Coleman, 1999).

Togetherness still plays an essential element in determining the quality of the immigrant-host's(s') and $V F^{\prime} s\left(s^{\prime}\right)$ social interactions. Examining the temporal dimensions of social interactions reveals that there is an intense period of social interaction that is preceded by and followed by little contact. This has been an observed pattern where the nature of friendships is separated by distance, particularly for the Filipino immigrants in New Zealand and their friends in the Philippines. The non-routine pattern of contact between friends where intense interaction occurs in the during-visit phase may be followed by an absence of regular contact. However, relationships between visiting and hosting friends are sustained and maintained over indefinite periods by individual nostalgia and through long-term social processes. The collected narratives of the participants reflect how their friendships had begun and had been enhanced and continuously nurtured through hosting/visiting and travelling. Therefore, in the context of hosting and visiting friends, emotional solidarity is maintained by the face-to-face interactions even though they may be irregular and defined, but nonetheless intense and meaningful.

\subsection{Cultural beliefs and behaviours on hospitality}

To address the fourth supplementary research question: "How does culture shape social interactions between the immigrant-hosts and VFs?", the immigrant-hosts were asked what were their hosting beliefs and behaviours and whether they still reflect or express that they are "Filipino." However, as immigrant families host friends in New Zealand, these families are also in the process of learning about their new homeland, and this transition in residency may impact on the way or manner they host their guests. Given 
that a familial perspective was solicited from the immigrant-hosts, an emerging theme in relation to the gender dimension of hosting will also be discussed and show how other members of the hosting household contribute to the social interactions. For new immigrants, the value and utility of local knowledge about their environs and about New Zealand were important aspects of their hosting behaviour.

As friends serve to validate identities (de Vries, 1996), immigrant-host families still perceive their hosting behaviour as being "Filipino" and VFs further reaffirm the notion of hospitality in the context of Filipino culture. Hospitality is not only a material relation (Lashley, Lynch, \& Morrison, 2006; Lynch et al., 2011; O'Gorman, 2010); it becomes central to pakikipagkapwa or humaneness which is at the core of Filipino culture in relation to others, including friends, which may be an unwritten obligation of the hosts and what their Filipino guests may be expecting from them when they visit New Zealand. Within Filipino culture, the generosity of a host becomes part of an essential element to their interpersonal relationships with their guests regardless of the personal cost, whether it is monetary or in effort.

In relation to the effect of culture on hosting, this may serve to explain the degree of sacrifice that a host will accept, such as temporarily surrendering one's privacy or the effort put in to helping to plan and organise the visit and activities of a friend. The manner of hosting within one's own culture may also be related to social exchange which involves the principle that one person does another a favour, and while there is a general expectation of some future return, its exact nature is definitely not stipulated in advance (Blau, 1986; Cook \& Rice, 2003). From a cultural perspective, social exchanges occurring through a visit may also be requested or imposed when dealing with a friend such as a visit where there is a request to host and a visit may not be entirely convenient for the host but they nevertheless agree to the visit out of a sense of respect or obligation and/or desire to deepen the status quo of the friendship. Therefore, the act of hosting can become a sacrifice which becomes almost obligatory as they appear to feel they have a duty to make these sacrifices and their guests may in turn expect the host to perform certain duties and favours. 
Examining how Filipino culture affects the social interaction between hosts and guests meant that both were asked about their perceived beliefs and behaviours on hosting friends in New Zealand. While the Halcon family may not have extra space to properly host as they were living at a studio-apartment at the time of their hosting, they agreed to accommodate their guest within their very modest sized home and gave up their privacy for a couple of days in exchange for the pleasure of reconnecting with a close friend. On the part of the visitor, while he may not have had the opportunity to explore Wellington to its fullest, the visit with his best friend was seen on his part as necessary in order to become acquainted with his childhood friend's family. The latter part of this section will disclose what the visit means to both the Halcon family and their guest. In a group interview, the Halcon family describe their hosting behaviour:

Host-wife: You have to be understanding, [and] flexible.

Host-husband: Flexibility, taking care of the wants and needs of the guest Host-wife: Being sensitive. Open-minded, also willing to sacrifice a bit of comfort for a few days and your privacy [giggles]

Meanwhile, the Halcon family's guest share the same insights:

Male visiting friend of Halcon family: They still accommodated me and then they wouldn't mind if I squeeze myself in their place. They would offer food and accommodation, that's it. Let us say for example, I compare it with the values of others like a friend who is married to a non-Filipino, it's unlikely that you'd ask them if you can stay in their place. [....] You're expected not to be able to stay at their place if there is a non-Filipino. For a Filipino, you have some sort of some hope, a level of hope that you will be adopted, quote and unquote, by your host. So for example, you can [even] sleep in their couch if they don't have guest room.

For the Mayon family, the act of hosting and making the extra effort to bring their guest from Auckland to Wellington, and even to Queenstown (even if unaccompanied but expenses paid by the immigrant-host family) underscores the kind of relationship they have formed more than three decades ago with their guest. The bond with the guest is also deepened by the fact that the guest is their godmother who is almost equivalent to being their kin. Even if the immigrant-host son of the Mayon family grew up in New 
Zealand and may not have a deeply significant relationship with their guest, which was began when his parents were still living in the Philippines, he is obliged to accord the same traditional Filipino hospitality being shown by his family, to their guest and in the manner that he observes every time he returns to his parent's former homeland. The importance of a return visit to the Philippines was also essential not only for the parents, but also for children who grew up in New Zealand in terms of socialising with their Filipino guest. This will also enable them to better understand Filipino culture and also when dealing with their parent's Filipino visitors.

I host as a Filipino - as a host, you know, I have been living in New Zealand for more than a decade - two decades, but still, like what I've seen, the values we have will be there forever. Wherever you go, it still will be there. Immigrant-host father, Mayon Family, individual interview

Maybe I showed the Filipino values of being hospitable and conforming with your guest. Immigrant-host mother, Mayon family, individual interview

A conversation with the immigrant-host son who was raised in New Zealand provided an opportunity to examine Filipino culture from the perspective of an individual who has observed and experienced hosting in the Philippines first-hand:

As I've seen other people who-when I stayed in the Philippines when I went to visit-some of the values that they have in the Philippines apply to us here, too. So, like showing them sightseeing and bringing them to shops and stuff like that. So, basically, what we, Filipinos do in the Philippines is the same thing we do here to them. Immigrant-host son, Mayon family, individual interview

In the Philippines, the Mayon family's visitor shares the same sentiment:

Female visiting friend: Well, the Filipino values [of the immigrant-host family] are still there such as being hospitable.

Interviewer: What is your definition of Filipino hospitality?

Female visiting friend: Like if you visit, they will accommodate you [and give] everything that they can or whatever they want to share.

With regard to Maria in the Pinatubo family, she paid for her friend's airfare from Auckland to Wellington, spent her whole weekend with her guest and introduced her friend to her life-partner which is an expression of her authentic self and being a true 
and valued friend. While there is a much greater affinity or cohesion between Maria and her guest due to their past interactions overseas, Leonora eventually felt in unity with the group by seeing the importance of her partner's hosting of a friend:

It's very Filipino that you provide whatever the best that you have for your guest. It means you have to use the best china. It means that you choose the best cut of meat that you can afford. Ah, that's very Filipino. What else? You have the tendency to show the good thing -- that's very Filipino. Good things, I mean, I am speaking generally, since in my case, I already know him. Maria, female immigrant-host, Pinatubo family, individual interview

I guess the standards of Filipino hosting is different [...] Say, when a guest tells you that they will be visiting, there is a sense of responsibility to make sure that the visitor is welcome in your house. Within the Filipino culture, you have to fit them in your schedule. Leonora, female immigrant-host, Pinatubo family, individual interview

Meanwhile, the notion of Filipino hospitality accorded by immigrant-hosts is echoed by the visiting friend of the Pinatubo family who considers himself as a guest:

Male visiting friend: Yes, definitely because they showed the usual way that we treat our guests.

Interviewer: What do you mean "the usual"?

Male visiting friend: They treat you out for dinners, lunch. And then free accommodation, right? And then they ask you where you want to go? So, I told them since I've heard that there was this nice museum in the city. However, time was not enough to go around. The museum was huge and I have to rush from one section to another. I also remember that the museum was free.

Interviewer: When you say "the usual way of treating our guest", do you mean to say the Filipino way?

Male visiting friend: The Filipino way.

As for the Ragang family, the host mother's selfless hosting of a friend indicates just how much she values her friendship with her best friend even if they have not seen each other face-to-face for a very long time. During the interview, she also mentioned that she even took leave from work while telling her guests that it was her day off so, 
that her guests would not worry that their host was placing her job in jeopardy or feel guilty that their host was sacrificing her leave time just to show them around. Similarly, this immigrant-host mother has accorded the same compassion not only to her best friend, but to the entire visiting group, even if the rest of the members may have different motivations to travel to New Zealand. She may not be materially well-off compared to her best friend (and the rest of the visiting group), yet she found ways to make the visit of her guests stimulating and pleasant, to the point of requesting her relatives in Taupo to assist in the hosting and asking her brother to drive her guests around the North Island (refer to Table 4.5):

I think my behaviour is still Filipino. You won't allow them [visitors] to leave empty-handed as you try to give something. You entertain them as much as possible and shoulder the [costs for the] meals - the typical Filipino wants to give everything to make their guests happy. Unlike the Kiwi [hosting a fellow Kiwi]; they frequently look after their own [bill] and [may] let the guest pay for themselves. For Filipinos, you must show some understanding [for visitors who travel such a long way]. Immigrant-host wife

Yes, [even] my mother is two thumbs up to my friend who exerted so much effort. She is even more excited to see the two of us together [with my friend] because of the friendship we have forged. Maybe, if I am just an ordinary friend, her hospitality would not be like that. Female visiting friend, group interview

They didn't show anything different. They're good. You can see that from them. They have never changed [as Filipino]. Mother of female visiting friend, individual interview

The Taal family guests were actually relatives of Mara's ex-partner and she showed her humility and respect by accepting to co-host her ex-partner's guests as visitors within their shared home. The traditional expectations of Filipino culture and the respect of the guest trumped Mara's personal needs or desires for privacy within her home. This selflessness demonstrates that despite being a resident of New Zealand, Mara still holds onto much of her native culture's values on hosting:

Mara: Pakikipagkapwa (humaneness) has relevance since it is imbibed in our culture. When you have friends or relatives coming over, it is part of our culture to be hospitable. So we have to always accommodate them and try to show them to different place and try to have the best experience with them - and for them to enjoy while they are here and [considering] they spent money to go here - so why not let them enjoy it? 
Like the other Filipino guests interviewed in the Philippines, Clara also shares the same view which makes her comfortable to approach her Filipina host:

Clara: Every Filipino host is hospitable and will take care of you.

Interviewer: What's your definition of hospitable?

Clara: Like, you don't have to think about anything.

Interviewer: You mean, when you are the guest?

Clara: Yes, you just sit there -- and anything will happen. They plan everything. And usually, when people visit here, when you (also) have guests here -- you plan everything -- take them everywhere.

Outside of the de facto relationship category of family, friendship also appears to be a special relationship to which people attach great personal and cultural importance not only providing one with a sense of identity, but also to confirm social worth (Allan, 1989). As immigrants establish their roots in New Zealand, hosting for a friend is also valuable for maintaining ties in the Philippines, especially for long-term residents. For immigrants who may not have relatives in their new homeland, maintaining connections with their friends seems to be essential to the majority of immigrant-hosts and VFs as their friends reflect their cultural beliefs and behaviours as Filipinos. As most immigrant-hosts and VFs have a common understanding of what hospitality means in the context of Filipino culture, both actors still express an act of pakikipagpalagayangloob or mutual trust. They are comfortable in dealing with one another and VFs do not feel hesitant to approach their immigrant-hosts and request to be accommodated and immigrant-hosts extend the invitation as an act of mutual trust and generosity. On the other hand, being true to their friendship allows the immigrant-hosts to let their guests know their hosting tolerance, which in the Filipino culture means hiya. For example, if a prospective host has less work during a particular month, they would inform a potential guest at the point of making the invitation that they could host, but only during that month long period. The guest would then understand that the offer is contingent on the visit lasting for a maximum period of one month and their host could comfortably tolerate their visiting during that time. However, a better understanding of New Zealand culture may be necessary or beneficial for immigrant-hosts to better undertake their duties to their friends. 
From a philosophical view, the phenomenon of hospitality necessarily contains the concept of the other or, the stranger, since hospitality requires, a priori, a concept of the outsider or guest (O'Gorman, 2010). However, this study shows that hospitality may also be part of one's cultural norms and expectations, within a particular group which the hosts and guests may still share. Specifically, immigrant-hosts and VFs provide insights in relation to their cultural beliefs and behaviours on Filipino hospitality in a multicultural setting, in particular, New Zealand. While a place may (still) be somewhat "foreign" for some recent immigrants and may be "strange" to their guests, the reflexive accounts of the immigrant-host families divulge how their hosting behaviours reflect their Filipino identity, which was substantiated by their VFs.

While attention has been drawn to aspects of hosting by families, a gender perspective is also provided as immigrant-host families have obligation to their VFs. As hosting occurs in a different setting, the cultural differences between the Philippines and New Zealand affect the hosting ability of migrants, particularly for new residents who may not have fully integrated within New Zealand's culture or are still financially struggling. Evidently, immigrant-host families are an integral part of the trips taken by their guests (as earlier discussed in section 4.3). However, there is a lack of a whole-family perspective that examines the broader experiential dimensions, sociality, and domesticity in order to understand the views of other family members (Schänzel, 2010a, 2010b) in relation to hosting for VFRs. This study attempts to give voice to other members of the immigrant-host family with regard to their hosting experiences.

When examining the group dynamics within the immigrant-host families, there is a definite gender dimension to the responsibilities in terms of the domestic duties of women in hosting versus the role(s) and responsibilities of male members of the hosting household. During a group interview with a host couple, they express different expectations in hosting. For instance, the immigrant-host wife in the Halcon family is expected to serve the needs of her husband's friend:

Host-husband: I think there are differences because I am thrifty [laughs]. 
Host-wife: I didn't know that you would mention that. Because for me, I'd rather buy prepared food than cook.

Host-husband: For me, I wanted to cook.

Host-wife: For me, I was not yet confident to cook as I am not familiar with the ingredients here.

Host-husband: But I'm confident with her [my wife's] cooking.

Host-wife: (Laughs) I told him that he is more proud of my cooking than I am.

While an immigrant-host family may now reside in New Zealand, a disproportionate division of household labour remains and more effort is expected of Filipino women, which may become a substantial burden as they assume additional domestic duties related to hosting in addition to work outside of the home. This is reflected by the Halcon family being new residents to New Zealand. The host-husband migrated in 2010 and received his permanent residency just prior to the time of interview. On the other hand, the immigrant-host wife moved at a much later stage, as she had to remain in the Philippines to care for their family, and she arrived just before bringing their children to New Zealand.

Filipino culture nurtures beliefs that tend to bind women to their traditional roles at home, performing the role of wife, mother, and housekeeper (Sobritchea, 2012). In relation to domesticity, the weight of hosting responsibility also becomes a great challenge for women, especially recently arrived female residents, as the host is assumed to be keenly familiar with the services and their respective locations at the destination. While the visit allows interconnectedness with the guest, immigrant-hosts are still going through the process of discovery and establishment in their new homeland. Immigrants may still be unfamiliar with their new communities in constructing a new home and sense of community (Griffin, 2013). For example, the immigrant-host wife's lack of local knowledge about New Zealand in relation to available resources made her feel somewhat anxious when anticipating the responsibility of preparing meals for their guest which was revealed in the following individual interview: 
Immigrant-host wife: So, I was a bit pressured. Oh God! I will be hosting for a guest and I don't know where to buy food or what to prepare for him. I don't even know what snacks to have or whether there are fruits available [laughs]. Then, there is the fact that when someone is visiting, I have this feeling [of doubt] whether I can serve him well.

Interviewer: You mean there is some hesitation on your part since you are not yet familiar with the place?

Immigrant-host wife: Yes, oh my! I was even thinking whether he'll be comfortable as we are staying in a studio-type of flat where we are on our [matrimonial] bed while he is on an air bed.

Interviewer: Between you and your husband, were you the one who often cooks?

Immigrant-host wife: Well, not always. It is just that my husband arrived in this country ahead of me so; he learned how to prepare food here in New Zealand. But, he does cook in the Philippines although we have someone to cook for us over there.

Interviewer: Would you say it was a transition period for you at that time?

Immigrant-host wife: Yes, while preparing [to host]. There was a bit of nervousness -- because everything was all new then and someone was coming as well. Something like that -- but at the same time, it was fun as someone from back home who was visiting. At least, I knew the person. It feels good to see a person who is familiar to you.

Nonetheless, the immigrant-host father expressed his sentiment as a new immigrant and his appreciation in hosting:

As a new resident, I was very busy working here in New Zealand and your objective if this is the case is not really to enjoy. I was only here to work and earn money to support my family. It (the visit) was an opportunity to learn about New Zealand deeper than what I used to know before the visit. Immigrant-host husband, Halcon family, individual interview

There is also a scope for a host family member to conform within the household in order to accommodate their guest(s) so they fit their hosting activity together with their employment:

Since we're hospitable by nature, even if it will create conflict in our lives or work, we still try to accommodate them anyway and allow them to enjoy the experience while they're here. Mara, female immigrant-host, Taal family 
However, this sentiment was not apparent from long-time residents such as the Mayon family or the rest of the long-time immigrant-host families. The ability of immigranthost families to be fully integrated within New Zealand society by having local knowledge of their new homeland will definitely enrich their social interactions with their VFs.

Meanwhile, the Pinatubo family, being a non-traditional family (lesbian couple), took turns with their hosting activities. Immigrants who have been living in New Zealand felt that having resided longer in New Zealand made them more familiar and therefore more at ease with hosting in their new country. There is a benefit of having increased local awareness as this aids immigrant families in more comfortably hosting their guests. As well, general knowledge of New Zealand is vital to providing enriching experiences when hosting for their friends:

I'd only been in the country a short while before I was able to determine that [how to host] -- but, it wouldn't make any difference except that I can find more things now here in New Zealand. Maria, female immigrant-host, Pinatubo family, individual interview

For me, I feel that I could have toured them around to many places. However, while we have been here for four and a half years, I have limited knowledge since I only know the places where the brother of my sister-in-law has toured us. If we are going outside North Island, I don't know since I haven't been there. Immigrant-host mother, Ragang family

While scholarly literature on VFR travel has pointed out the importance of the familiarity of hosts with tourism products (e.g., Brocx, 2003; Slater, 2002), there is a need to re-examine hosting in the context of immigrants, particularly in the context of newer residents. Indeed, having local knowledge of New Zealand is important for immigrant-host families. As domestic hospitality (Pilardi, 2010) occurs in the context of VFR travel, at least for those who were accommodated by the hosts in their residence, a gendered dimension of the social interactions between immigrant-hosts and VFs shows that hosting should be a collective duty of immigrants to meet their guest's(s') needs. However, hosting as a domestic responsibility typically becomes a 
heavy burden upon females (host-wife) within the hosting household, even if she is only a secondary host for her husband's friend. Therefore, the familiarity of the host wife with regard to accessing food in their new homeland and the coordinating of domestic tasks of other members of the hosting household are essential to successful hosting and these functions typically do not become an additional burden for other members of the hosting household when hosting for their friends.

In this light, examining the earlier interaction of immigrant-hosts and VFs from a cultural lens provides various insights. There is a common belief about the notion of Filipino hospitality among the immigrant-host families which seems prescriptive and the Tagalog term for host is punong abala which is loosely translated as "primarily busy, preoccupied, or bothered." The act of hosting and visiting is a way of sharing oneself which is seen in the Filipino way of life. However, it is limited to one's "in-group" or, those to which one has personal relationships. Within one's group, sharing is not merely dictated by pressure, but it is voluntary (Babor, 2007; Quintin, 1981).

\subsection{Meanings of the visit among friends}

The act of hospitality being shown by immigrant-hosts is principally a result of their friendships being shaped by continuing social exchanges and emotional solidarity as they show their respect and affection for their Filipino guests in New Zealand. Underpinning the act of hospitality are the meanings of social interactions, including hosting and visiting. From the foregoing section, the perspectives regarding Filipino hospitality from the point of view of both the hosts and the guests are presented, which validates their shared identities as friends. The component that examines Filipino culture is now integrated into the meanings of the social interactions between the hosts and the guests and was again triangulated for each case. This addresses the main research question for this chapter: "How are social interactions between immigranthosts and their visiting friends understood and interpreted by them?

Each social interaction has a different meaning for each participant. The meanings vary depending on their perceived value of the friendship that was established between a 
specific immigrant-host family member and their respective guest/s. However, a common understanding between the social interactions of the immigrant-hosts and VFs elicit an on-going mutual trust and commitment through one's co-presence, reacquaintance, and re-establishing of ties. Significantly, what is another apparent meaning for immigrant-hosts is that they may also develop a sense of pride from hosting in their new homeland while secondary host(s) (other members of the family) and other ancillary member(s) of the principal guest's party may foster solidarity through the visit.

The co-presence of friends requires the opportunity to meet and spend time together as occasional physical proximity is supportive and helps to sustain the relationship. For example, the principal host for the Halcon family (the immigrant-host husband) separates or classifies "common friend" from a "best friend" where the former may be categorised as an "acquaintance" which means that persons have an ongoing affiliation that may be less intimate or significant when compared with the meaning of the latter term:

I even have a friend who visited Auckland and wanted to come to Wellington. Eventually, things did not push through because our friendship is not that strong. I think the person was able to come to the city but did not contact me anymore - probably because I was busy at work. But for my recent guest, even if my place is tiny, I would not hesitate to host even if I have limited resources, without any fuss. Of course, I told him what he would be expecting when he arrives. Immigrant-host husband, individual interview

As the secondary host within the Halcon family, the immigrant-host wife, felt in solidarity with the friendship of her husband and the guest:

Well, it was very important knowing as he's my husband's friend. It's also good for him to see us as he was in Auckland. Immigrant-host wife, individual interview

Friendship may also mean the sharing of one's time during travel to visit a friend overseas, to re-establish ties, despite having other initial motivations for the trip:

Ah, very important [to visit them] plus the fact that I was [already] in New 
Zealand [after attending a conference] - I love to see them, so in fact, if for example, if [host friend] does not live in Wellington, maybe I would not have dropped by Wellington. Maybe, I'd find an Auckland flight directly to Christchurch. Male visiting friend of Halcon family

For the Mayon family, the importance of co-presence, returning the favour, and making the guest feel at home in New Zealand are essential to the immigrant-host family, and for the guest to re-acquaint with her hosts:

It's very, very important, you know, as we say that we have to be with somebody, not by emails or phone but something physical, right there in front of you is different from somebody you just ring on the phone. Immigrant-host father, individual interview

The visit is important because when we were still in the Philippines, our friend also hosted and accommodated us. It's an opportunity to return the favour. Immigrant-host father, individual interview, individual interview

For us, it's quite important because say if we weren't here, and say if we live in Auckland and she came down here, it makes her feel by herself, so, at least, were down here, we hosted her and we made her feel at home while even showing her around Wellington. Immigrant-host son, individual interview, individual interview

Well, I also miss them, so, it's very important for me to see them, to make kwento [share stories] with them -- and your bonding with each other. Female visiting friend

For the Pinatubo family, hosting and visiting a friend is vital to re-acquaintance and the rekindling of their friendship that was forged a long time ago. Even new-found friendships were established during the visit, particularly for a specific member of the hosting family (being the secondary host), finding fellowship upon meeting their guest for the first time:

We are connecting; we are engaging [...] It is also important, as if you wanted to show where you are now, not necessarily what you have achieved, but your new environment. [...] You give them a peace of mind that you're okay now --"she is all settled." If you haven't seen a friend or family for a while, they will always wonder or worry -- "how are your friends or your family doing?" Maria, female immigrant-host, Pinatubo family, individual interview

The visit was important as our guest is special to Maria being her friend. Leonora, female immigrant-host, Pinatubo family, individual interview 
First of all, I haven't seen her for a long time. I have to renew my ties. I wanted to know anything new about her. What happened to her in between (those times that we parted ways). You know, rekindling ties with friends. Of course, friends that you value -- because there are also types of friends that it's alright not to see them. Male visiting friend of Pinatubo family

Hosting and visiting friends also re-establishes ties and holds a promise to visit other places in the future. On the other hand, this may also mean achieving other purposes for other members of the visiting group:

Her visit was very important since we became closer. If she comes back next year, which I don't know, I want to prepare and file a leave ahead of time. Her plan is for us to go on a ferry tour to Coromandel. I told her I'll check my schedule whether I am entitled for leaves. Immigrant-host wife, Ragang family

It is important to go there as a friend because not only would I see the place, but I would be able to see her and be with her. I have established a close relationship with her. Female visiting friend of Ragang family

Of course, very important [to visit New Zealand]. My brother has become angry with me due to a long pending invitation to visit him. Instead, we visit to other countries instead of him. He was very happy. Regarding [female host friend], when my daughter saw her, both of them were really happy. Mother of female visiting friend of Ragang family

Hosting can also impart a sense of fulfilment for a host as one takes pride when friends appreciate their new home, and conversely, for the guest to achieve their other motives to meet others and to social network in New Zealand:

For me, I felt instrumental to them loving it here and enjoying the experience. Although somehow, it's a strain - time, resources. When you hear feedback that they were happy and they would like to return except that Uncle has a physical disability, then, I told myself I became instrumental for them to appreciate New Zealand. Mara, female immigrant-host, Taal family

Well, it's really important like I also visited as well my classmates. She's in Auckland and she's working there. I visited her and we had dinner. Clara, female visiting friend of Taal family 
In constructing the meanings of their social interactions, VFs have also defined their role and identity as guests in most cases. However, there are also mixed responses coming from VFs which can lead to various interpretations based on the context of the particular interlocutor:

No [I don't feel like a visitor], because it is very easy for me to tell what I like. So, I told her, I want to try this - and because before I went there, I did some research. I told her [my best friend] that I want to have a cruise in Auckland ferry. It was also her first time to be there. So, that's a normal way. If we have visitors, we want to show them around. So, it was a first time for her because all her time is devoted to work or at home. They don't go out that much. Female visiting friend of Ragang family, individual interview

[As guest] We are ashamed to be hosted. We wanted to pay our bills, but they refuse. Mother of female visiting friend of Ragang family, individual interview

Within this group of VFs, there are occasional differing or conflicting responses that may indicate a different point of view between them in relation to how they interact with their host. The female visiting friend appeared to be comfortable in asking favours of her host family as she is the best friend of the immigrant-host mother. On the other hand, the lack of familiarity or closeness of the mother of the female visiting friend with the host family makes her hesitant to ask for favours of any kind or demand anything beyond the apparent means of the host. The differing responses between these guests would be due to the fact that one guest was a close friend and the other was her mother and travelling companion. Thus, the accompanying mother felt social discomfort or conflicted in accepting the generosity of the hosts as she did not have close ties to the hosts prior to the visit with the friends of her daughter.

Membership in social groups or collectives provides an important basis for selfdefinition. In addition to whatever traits or characteristics people use to describe themselves as unique individuals (sometimes referred to as personal identity), they also locate themselves in the social context (Deaux, Reid, Mizrahi, \& Ethier, 1995, p. 280). Under such circumstances such as being a guest therefore may also depend on where a person stands in relation to their host as described below: 
Interviewer: So, how would you describe your role during your visit to Mayon family?

Female visiting friend: My role? Maybe as a visitor or part of the family. I feel it that way because they are close to me.

Interviewer: So, how would you describe the hosting of your friend in New Zealand?

Female visiting friend: It's okay. Everything is provided. I cannot ask for anything more.

Interviewer: And considering what you have told me, do you see yourself as a guest, why or why not?

Female visiting friend: If I consider myself as a guest, maybe because I am new to that place. The fact that I am new, I consider myself as a guest. The fact that I am new [to the place], of course, they have to tour me. They have to show me everything. They provide what I need, food and lodging. It's a feeling that I am one with the family.

Interviewer: You feel that you are one with them?

Female visiting friend: Yes, one of them.

In contrast, the immigrant-host son for the Mayon family has a different perspective about being a host to their visiting friend:

Immigrant-host son: I do not know if I had a role, really, it is just like the son. My Mom and Dad probably were the most of the host to her.

Interviewer: So, considering what you have told me about your interaction with do you consider yourself as a host?

Immigrant-host son: In some way, yes, because I showed her around, but not like a "total host", that would be Mom and Dad.

Since the visiting friend of the parents in the Mayon family was their godmother during their wedding, there is also a shared belief of inter-generational connectedness within the rest of the immigrant-host family members who also seem to own their friend as if they were their kin: 
Especially because my friend is now with my children, particularly with my youngest son...he is very happy to see our guest since she doesn't have a lola (grandmother) in New Zealand as my mother passed away before we migrated. Immigrant-host mother, Mayon family, group interview.

Being a primary sponsor during the wedding of the couple in the Philippines, the guest also becomes a co-parent even for the children in Mayon family in New Zealand and as such a spiritual relationship is developed through ritual kinship. In this manner, a group based on human relatedness that transcends blood relationship emerges. Being a godparent means one was chosen because of wisdom and character and they are therefore recruited as an intimate friend whom a family can trust and eventually become in solidarity.

Overall, the accounts in this section relate to the different meanings and understandings of the social interactions of the immigrant-hosts and VFs which, evoke social exchanges through an on-going mutual trust and commitment forged overseas as they continue showing concern, sympathy, and openness in a different setting. Emotional solidarity enhances friendships through one's co-presence, re-acquaintance, and re-establishing friendship ties as hosting and visiting provides an opportunity for friends to spend time together since periodic or even sporadic physical proximity is critical in sustaining friendships across time and distance. Such social interactions are accorded as friends can still identify themselves with their significant others despite the immigrant-host now living overseas and being immersed in a different cultural setting that is far from their friends in the Philippines. When self and identity (as friends) are confirmed in situations, individuals experience positive emotions, whereas when self and identity are not confirmed, individuals feel negative emotions and are motivated to seek confirmation (Stets \& Turner, 2006). Accordingly, hosting and visiting friends become a special responsibility as mutuality exists between friends which one does not extend when dealing with strangers. This may also produce a fellowship in meeting new found friends, particularly between a secondary host and a VF, or between a primary host and other members of the visiting group. 
The pattern of interaction and understandings gives rise to legitimate expectations about personal characteristics such as caring, support and honesty, among others. This is true of particular friendships, but also true of friendship as a general pattern of interactions (Annis, 1987). Overall, hosting and visiting for Filipino immigrants and their friends from the Philippines, respectively, becomes an obligation that is done face-toface which cultivates caring, cooperation, commitment, good will, and loyalty that is hoped by all concerned parties to be maintained across time and distance.

\subsection{Conclusion}

When investigating of the main question: "How are social interactions between immigrant-hosts and their visiting friends understood and interpreted by them?", exploring the social interactions of the immigrant-hosts and VFs reveals that they are quite complex which generates a range of themes encompassing time (the temporality of social interactions), meanings of social interactions (past interactions, reacquaintance, importance of hosting/visiting), persons involved (immigrant-hosts, VFs, other social networks), places (multi-destination trips and itinerant guests), and culture (identity and relationships). Through hosting and traveling, immigrant-host's(s') and $V F^{\prime} s\left(s^{\prime}\right)$ social interactions are continuously maintained but are periodically enriched and nurtured through visiting and their mutual social exchanges are not based on proximity, but are continued intermittently over a period of time. Examining immigrant-host's(s') and VF's(s') social interactions across time and space also shows how emotional solidarity fosters VF travel through the co-presence of friends.

Both hosting and visiting in the context of VF travel involves social exchanges based on mutual trust, commitment and loyalty of friends. The social interactions between them also engender a feeling of personal obligation which may also be costly while at the same time rewarding. Considering the past interactions of the immigrant-hosts and VFs, social interactions from hosting and visiting provided a context for the on-going social exchanges between friends which are maintained through the years and across greater distances. As social networks are becoming increasingly dispersed (Larsen et al., 2007; Schänzel, 2013; Sheller \& Urry, 2004; Urry, 2002a), VF travel provides 
opportunities for Filipino immigrants and their friends to be together within the context of the tourism-migration nexus. Most of the Filipino immigrants are still maintaining their friendship with their guests, which becomes intense at the duringvisit phase compared with the other temporal dimensions of their social interactions, in particularly the pre- and post-visit phases. Likewise, there can be an uncertainty for friends to meet again in the immediate future as reflected earlier by their post-visit social interactions.

Friendship is a social reality which reflects and reinforces wider social realities. It is shaped by recreating and maintaining social interactions. Through VFR travel, social interactions occurring through the co-presence of friends establishes one's identity whether in expressing hospitality, culture, or friendship. Given that social interactions in the during-visit phase provides a shared experience for both hosts and guests that is made tangible for them, hosting and visiting friends becomes essential which provides several meanings through their shared experiences.

A gendered dimension of hosting also reveals an interesting insight with regard to domesticity and familiarity, as hosting becomes mainly a part of domestic duties of immigrant-host mothers. In the context of whole-family research (Schänzel, 2010b), the voices of other members are included as they share the meanings of their hosting/visiting experiences. Local and general knowledge of New Zealand benefits immigrant-hosts families when hosting, particularly when accommodating a guest in their residence where the commensality of food becomes necessary. Friendship appears to be a special relationship to which people attach great importance, both personally and culturally, as friends provide a sense of being and confirm social identity.

Indeed, the immigrant-host families and their VFs continue to share the same beliefs and behaviours with regard to hosting and visiting. Further research on the meanings of friendships in other societies and how it relates to VF travel may be useful to understand the social interactions of immigrant-hosts communities living in their new 
homeland. From a practical view, a longitudinal study may be useful to examine to determine if VFs are returning to New Zealand and are still being hosted to maintain their friendship with their respective immigrant-hosts, considering that all of the interviewed VFs were first-time visitors. Following this analysis and interpretation of the immigrant-host's $\left(s^{\prime}\right)$ and visiting friend's $\left(s^{\prime}\right)$ social interactions, the following chapter will address the social interactions between immigrant-hosts and their visiting relatives and will follow the same presentation in terms of the key findings related to kinship. 


\section{CHAPTER 5 - Analysis of the social interactions between immigrant-hosts and visiting relatives}

Analysis of the home and social dynamics therein would lead to a greater understanding of the socialisation and domestication processes and practices that condition, and transmit hospitality behaviour, norms, values, meanings and expectations between generations. (Lashley et al., 2006, p. 190)

\subsection{Introduction}

The quotation above positions this chapter in relation to the analysis of the social interactions between immigrant-hosts and VRs, specifically in the context of Filipino immigrants in New Zealand and their relatives from the Philippines. In particular, this section discusses how social interactions reflect social exchanges and emotional solidarity within the context of New Zealand, particularly for the immigrant-hosts and their guests (VRs) who are related to one another. They may share common beliefs and behaviours (given their family bonds, a previous common nationality and shared ethnicity) but could hold different views and perspectives that may be the result of experiential differences (such as migration to New Zealand).

This chapter addresses the main research question in the context of the social interactions between immigrant-hosts and VRs: "How are social interactions between immigrant-hosts and their visiting relatives understood and interpreted by them?" This question is addressed by first presenting the four supplementary questions: (1) What is the nature of social interactions between the immigrant-hosts and VRs? (2) What are the social exchanges between the immigrant-hosts and their VRs? (3) How do social interactions reflect emotional solidarity between the immigrant-hosts and VRs as expressed by their shared beliefs and behaviours? and finally (4) How does culture shape social interactions between the immigrant-hosts and VRs?

This chapter explores the social interactions of immigrant-hosts and VRs in terms of two theoretical frameworks: social exchange theory and the theory of emotional solidarity. These two theories provide the conceptual foundation to examine the 
phenomenon and to understand the complexities of the relationships between immigrant-hosts and VRs in a cultural context. These theoretical frameworks show distinct ways of understanding social interactions in the context of the cultural identities, exchanges, beliefs, and behaviours of first-generation migrants in New Zealand and their visiting relatives from the Philippines. The structure of this chapter parallels the specific research questions presented earlier. Section 5.2 provides background about the social interactions between immigrant-hosts and VRs which addressed the first supplementary research question. Fundamental to addressing the second supplementary research question related social exchange (section 5.3), social exchanges within the family are characterized by interdependence between immigrant-hosts and VRs. These social exchanges involve reciprocity and are manifested in caring for one another and mutual obligation as an important responsibility of the hosts and guests.

The third supplementary research question relates to emotional solidarity and is discussed in section 5.4 (Familial solidarity: social identities and shared social interactions). This section discusses how emotional solidarity is expressed through the shared identities of the immigrant-hosts and VRs and how everyday interactions become extraordinary for them. Section 5.5 (Cultural identities in New Zealand) addresses the fourth supplementary research question and provides an understanding of social interactions in terms of how the context of culture influences the emotional intimacy and social exchanges. The last section (section 5.6: Meanings of the visit among relatives) addresses the main research question for this chapter and illustrates the meanings that the participants attach to their social interactions which they socially construct and interpret. The dimensions of social exchange, emotional solidarity, culture and the interpretations of immigrant-hosts and VRs are then integrated in the concluding part of this chapter (section 5.7). 


\subsection{The nature of the visit}

Addressing the first supplementary research question: "What is the nature of social interactions between the immigrant-hosts and VRs?", Table 5.1 provides the characteristics of the immigrant-host families and their visiting relatives, such as the composition of the host family, the relationship of the host/guest, the residency period of the immigrant-host families in New Zealand and the duration of the visit by their relatives. Unlike some of the visits of VFs where they were identified as being "itinerant guests", almost all of the VRs stayed with their hosts during their entire visit to New Zealand and for significantly longer periods of time. Visiting relatives usually had a stay lasting from one to twelve months depending upon the specific motivations or obligations to host or visit in New Zealand. The residency of immigrant-host families in New Zealand ranges from five to more than 15 years. Another major factor that incites VR travel is child care and, except for the Arayat and Isarog families who were longtime residents to New Zealand, most of the participants interviewed had younger families consisting of infants, toddlers or primary or high school aged children.

Five out of the eleven groups of visiting relatives stayed for six months which is the maximum allowable period given by Immigration New Zealand for visitors from the Philippines. In addition, most of the VRs interviewed were return visitors to New Zealand; only the guests of the Banahaw, Matutum and Penablanca families were firsttime visitors to New Zealand. The siblings of the two immigrant-host daughters for the Guiting-guiting and Isarog families were also first-time visitors, but their respective visiting parent(s) had made previous visits. All the VRs interviewed were consistently and unvaryingly accommodated in the residence of the immigrant-host families for the entirety of the visit and no attempts were made to promote any side trips of the VRs that would separate them from their principal hosts. This would indicate that VR travel has a common and fundamental purpose: reunification of family. It would appear that the intention of VR travel is re-establishing family bonds surrounded by ancillary communal activities that support and reflect the main theme of the visit of VRs, family reunification. 
The anthropological composition of VRs typically follows a matrilineal pattern as the majority of the principal hosts are the immigrant-host mothers in New Zealand. When classifying a participant in a social interaction, the researcher uses the reference of relationship of the guest to the principal host within the family, particularly the blood relationships existing between them. For instance, most of the immigrant-host mothers may also be referred to here as 'immigrant-host daughter/s' in relation to their visiting parent/s. Female hosts are mothers to their own children but also children to their parents who come as guests. Most of the immigrant-host fathers/husbands interviewed were then called 'immigrant-host son-in-laws' in reference to their relationship with their parent(s)-in-law. However, the terms may be used interchangeably (e.g. immigrant-host mother/immigrant-host daughter; immigranthost father/immigrant-host son-in-law; immigrant-host son/immigrant-host grandson; visiting mother/visiting grandmother) depending on the point of reference within a particular sentence. For families whose children were not interviewed, a couple may be referred to as immigrant-host husband/wife, similar to how it was presented in Chapter Four (the composition of members for the immigrant-host family was detailed in Chapter 3, Table 3.2).

Within the setting of the immigrant-host's $\left(s^{\prime}\right)$ and visiting relative's(s') social interactions, their motivations were interpreted as commonly remembered by the host(s) and VR(s). However, other motivations may have emerged during the course of the analysis. Contextualising the visit, the five main purposes of the visit were identified (which will later be fleshed out in sections 5.2.1 to 5.2.5) are: (a) childcare; (b) family visit; (c) eldercare; (d) celebration; and (e) assistance with prospective employment by a host for a visiting relative. Using Backer's (2012a) VFR definitional model in Chapter 2 (Figure 2.1), the earlier categories from (a) to (d) may be assumed to be "pure" VFRs or PVFRs, or those who stay with friends and relatives and state VFR as their main purpose visit, and since all the VRs were accommodated by their hosts they would hence be PVFRs. The other two aspects of the VFR model are: CVFRs (those who stay in commercial accommodation and came to the destination with VFR as a purpose of the trip); and EVFRs (identified as "exploiting" VFRs or those staying with their 
relatives/friends but visiting them is ancillary to the main purpose for their travel). However, what could be viewed as problematic is whether the last category, letter (e): assistance with prospective employment, should categorically be labelled as EVFR or "exploiting" VFRs. Should staying with relatives and having another purpose than that of a family visit where the VR may have pure and honest intentions despite having another motivation to travel other than solely a visit with their family be labelled "exploiting" VFRs? The issue of whether the other party was exploited or abused, and that one benefits over the other, is a question that will depend on the perspective and cognisance of the immigrant-hosts with respect to the EVFRs' motivation(s).

Therefore, when examining the social exchanges, emotional solidarity, and the cultural context of the social interactions between the immigrant-hosts and VRs, sensitivity and discretion may be necessary so as to not impugn the character of the research participants. Research should focus on the role of personal relationships in influencing motivations and behaviour. Hence, a more holistic view of examining motivations (Griffin, 2012; Riley \& Love, 2000) is to look at the perspectives of both immigrant-hosts and visiting relatives.

In the succeeding subsections, the individual perspectives of both the immigrant-host families and their respective guest/s in relation to the purpose of the visit (or motivations) are paired and the results shown from sections 5.2.1 to 5.2.4 are almost the same, as there may be a familial consensus in acceptance of the role of hosting/visiting relatives. However, there is also a scope for differing perspectives among family members. Section $\mathbf{5 . 1 . 5}$ is discussed separately as it deviates from the nature of the visit since the purpose of the visit of the guest is to look for employment opportunities while being accommodated by a relative. 
Table 5.1. Biographical information on the immigrant-host families, vis-à-vis their relative's(s') visit.

\begin{tabular}{|c|c|c|c|c|c|c|c|}
\hline $\begin{array}{c}\text { Immigrant } \\
\text {-host } \\
\text { Family } \\
\text { Name }\end{array}$ & $\begin{array}{l}\text { Years of } \\
\text { residency }\end{array}$ & $\begin{array}{l}\text { Composition of } \\
\text { immigrant-host family } \\
\text { members }\end{array}$ & $\begin{array}{l}\text { Motivation of the } \\
\text { Immigrant-host }\end{array}$ & $\begin{array}{l}\text { Composition of visiting } \\
\text { relative(s) }\end{array}$ & $\begin{array}{l}\text { Motivation } \\
\text { of the VR }\end{array}$ & $\begin{array}{l}\text { Duration of } \\
\text { relative's }\left(s^{\prime}\right) \text { visit } \\
\text { with the host } \\
\text { family* }\end{array}$ & $\begin{array}{l}\text { First-time visit of } \\
\text { the VR to New } \\
\text { Zealand } \\
\text { (Yes or No) }\end{array}$ \\
\hline \multirow[t]{3}{*}{ Pulag } & \multirow[t]{3}{*}{6} & $\begin{array}{l}\text { Immigrant-host mother/ } \\
\text { daughter of VR }\end{array}$ & $\begin{array}{l}\text { childcare for VR to look } \\
\text { after in view of a return } \\
\text { trip to the Philippines }\end{array}$ & \multirow[t]{3}{*}{$\begin{array}{l}\text { Visiting mother of } \\
\text { immigrant-host daughter }\end{array}$} & \multirow[t]{3}{*}{ family visit } & \multirow[t]{3}{*}{1 month } & \multirow[t]{3}{*}{ No } \\
\hline & & Immigrant-host father & family visit & & & & \\
\hline & & Immigrant-host son & family visit & & & & \\
\hline \multirow[t]{2}{*}{ Banahaw } & \multirow[t]{2}{*}{7} & $\begin{array}{l}\text { Migrant-host husband/ } \\
\text { son of VR }\end{array}$ & family visit & \multirow[t]{2}{*}{$\begin{array}{l}\text { Visiting mother of } \\
\text { immigrant-host daughter }\end{array}$} & \multirow[t]{2}{*}{ family visit } & \multirow[t]{2}{*}{1.25 months } & \multirow[t]{2}{*}{ Yes } \\
\hline & & Immigrant-host wife & family visit & & & & \\
\hline \multirow[t]{4}{*}{ Matutum } & \multirow[t]{4}{*}{6} & $\begin{array}{l}\text { Immigrant-host mother/ } \\
\text { daughter of VRs }\end{array}$ & family visit & \multirow[t]{2}{*}{$\begin{array}{l}\text { Visiting father of } \\
\text { immigrant-host daughter }\end{array}$} & \multirow[t]{4}{*}{ family visit } & \multirow[t]{4}{*}{2 months } & \multirow[t]{4}{*}{ Yes } \\
\hline & & Immigrant-host father & family visit & & & & \\
\hline & & Immigrant-host son & family visit & \multirow{2}{*}{$\begin{array}{l}\text { Visiting mother of } \\
\text { immigrant-host daughter }\end{array}$} & & & \\
\hline & & Immigrant-host daughter & family visit & & & & \\
\hline \multirow[t]{4}{*}{ Isarog } & \multirow[t]{4}{*}{$>15$} & $\begin{array}{l}\text { Immigrant-host } \\
\text { mother/daughter of } \\
\text { visiting parents }\end{array}$ & wedding celebration & $\begin{array}{l}\text { Visiting father of } \\
\text { immigrant-host daughter }\end{array}$ & $\begin{array}{l}\text { wedding } \\
\text { celebration }\end{array}$ & \multirow[t]{4}{*}{1 month } & No \\
\hline & & Immigrant-host father & wedding celebration & $\begin{array}{l}\text { Visiting mother of } \\
\text { immigrant-host daughter }\end{array}$ & $\begin{array}{l}\text { wedding } \\
\text { celebration }\end{array}$ & & No \\
\hline & & Immigrant-host son A & wedding celebration & \multirow{2}{*}{$\begin{array}{l}\text { Visiting sister of } \\
\text { immigrant-host mother }\end{array}$} & \multirow{2}{*}{$\begin{array}{l}\text { wedding } \\
\text { celebration }\end{array}$} & & \multirow[t]{2}{*}{ Yes } \\
\hline & & Immigrant-host son B & wedding celebration & & & & \\
\hline \multirow[t]{2}{*}{ Apo } & \multirow[t]{2}{*}{5} & $\begin{array}{l}\text { Immigrant-host wife/ } \\
\text { daughter of VR }\end{array}$ & child care & $\begin{array}{l}\text { Visiting mother of } \\
\text { immigrant-host wife }\end{array}$ & child care & \multirow[t]{2}{*}{6 months } & No \\
\hline & & Immigrant-host husband & child care & $\begin{array}{l}\text { Visiting auntie of } \\
\text { immigrant-host wife }\end{array}$ & child care & & Yes \\
\hline
\end{tabular}

*Note: Unlike VFs, the majority of the VRs reside with their respective immigrant-host family during their stay in New Zealand. 
Table 5.1. - Continuation.

\begin{tabular}{|c|c|c|c|c|c|c|c|}
\hline $\begin{array}{l}\text { Immigrant } \\
\text {-host } \\
\text { Family } \\
\text { Name }\end{array}$ & $\begin{array}{l}\text { Years of } \\
\text { residency }\end{array}$ & $\begin{array}{c}\text { Composition of } \\
\text { immigrant-host family }\end{array}$ & $\begin{array}{l}\text { Motivation of the } \\
\text { Immigrant-host }\end{array}$ & $\begin{array}{l}\text { Composition of visiting } \\
\text { related }\end{array}$ & $\begin{array}{l}\text { Motivation of } \\
\text { the VR }\end{array}$ & $\begin{array}{l}\text { Duration of } \\
\text { relative's(s') visit } \\
\text { with the host } \\
\text { family* }\end{array}$ & $\begin{array}{l}\text { First-time visit } \\
\text { of the VR to } \\
\text { New Zealand } \\
\text { (Yes or No) }\end{array}$ \\
\hline \multirow[t]{2}{*}{ Balbalasan } & \multirow[t]{2}{*}{6} & $\begin{array}{l}\text { Immigrant-host wife/ } \\
\text { daughter of VR }\end{array}$ & child care & \multirow{2}{*}{$\begin{array}{l}\text { Visiting mother of } \\
\text { immigrant-host } \\
\text { daughter }\end{array}$} & \multirow[t]{2}{*}{ child care } & \multirow[t]{2}{*}{6 months } & \multirow[t]{2}{*}{ No } \\
\hline & & Immigrant-host husband & child care & & & & \\
\hline \multirow[t]{3}{*}{ Arayat } & \multirow[t]{3}{*}{$>15$} & $\begin{array}{l}\text { Immigrant-host mother/ } \\
\text { daughter of VR }\end{array}$ & family visit & $\begin{array}{l}\text { Visiting father of } \\
\text { immigrant-host } \\
\text { daughter }\end{array}$ & family visit & \multirow[t]{3}{*}{6 months } & \multirow[t]{3}{*}{ No } \\
\hline & & Immigrant-host father & $\begin{array}{l}\text { for VRs to look after their } \\
\text { daughter }\end{array}$ & \multirow{2}{*}{$\begin{array}{l}\text { Visiting mother of } \\
\text { immigrant-host } \\
\text { daughter }\end{array}$} & \multirow[t]{2}{*}{ family visit } & & \\
\hline & & Immigrant-host daughter & $\begin{array}{l}\text { - family visit } \\
\text { - to be accompanied by VRs } \\
\text { due to parents return trip to } \\
\text { the Philippines }\end{array}$ & & & & \\
\hline \multirow[t]{2}{*}{$\begin{array}{l}\text { Guiting- } \\
\text { guiting }\end{array}$} & \multirow[t]{2}{*}{6} & $\begin{array}{l}\text { Immigrant-host wife/ } \\
\text { daughter of VR }\end{array}$ & family visit & $\begin{array}{l}\text { Visiting mother of } \\
\text { immigrant-host } \\
\text { daughter }\end{array}$ & child care & 6 months & No \\
\hline & & Immigrant-host husband & family visit & $\begin{array}{l}\text { Visiting sister of } \\
\text { immigrant-host wife }\end{array}$ & family visit & 1 month & Yes \\
\hline \multirow[t]{2}{*}{ Makiling } & \multirow[t]{2}{*}{6} & $\begin{array}{l}\text { Immigrant-host wife/ } \\
\text { daughter of VR }\end{array}$ & $\begin{array}{l}\text { - child care } \\
\text { - bereavement }\end{array}$ & \multirow{2}{*}{$\begin{array}{l}\text { Visiting mother of } \\
\text { immigrant-host } \\
\text { daughter }\end{array}$} & \multirow[t]{2}{*}{$\begin{array}{l}\text { - childcare } \\
\text { - bereavement }\end{array}$} & \multirow[t]{2}{*}{6 months } & \multirow[t]{2}{*}{ No } \\
\hline & & Immigrant-host husband & - child care & & & & \\
\hline \multirow[t]{2}{*}{ Penablanca } & \multirow[t]{2}{*}{7} & $\begin{array}{l}\text { Immigrant-host husband/ } \\
\text { nephew of VR }\end{array}$ & family visit & \multirow{2}{*}{$\begin{array}{l}\text { Visiting auntie of } \\
\text { immigrant-host } \\
\text { husband }\end{array}$} & \multirow[t]{2}{*}{$\begin{array}{l}\text { seek } \\
\text { employment }\end{array}$} & \multirow[t]{2}{*}{9 months } & \multirow[t]{2}{*}{ Yes } \\
\hline & & Immigrant-host wife & family visit & & & & \\
\hline \multirow[t]{2}{*}{$\begin{array}{l}\text { Sierra } \\
\text { Madre }\end{array}$} & \multirow[t]{2}{*}{6} & $\begin{array}{l}\text { Immigrant-host mother/ } \\
\text { daughter of VR }\end{array}$ & elder care & \multirow[t]{2}{*}{ Visiting father } & \multirow[t]{2}{*}{ family visit } & \multirow[t]{2}{*}{12 months } & \multirow[t]{2}{*}{ No } \\
\hline & & Immigrant-host son & family visit & & & & \\
\hline
\end{tabular}

* Note: Unlike VFs, the majority of the VRs reside with their respective immigrant-host family during their stay in New Zealand. 


\subsubsection{Childcare}

The primary motivation for the Apo, Balbalasan and Makiling families to invite their guest/s is a need for assistance with child care. Common among these young immigrant families in New Zealand are: (a) both spouses of the host couple have to work in order to earn an income suitable for the family to have a comfortable living to Western standards; (b) guest(s) were a principal relative of the immigrant-host mother which follows a traditional matrilineal pattern; (c) guest(s) stayed for a maximum of six months and are return visitors to New Zealand; and (d) a consensus of immigrant-host fathers in terms of the motivations to host for their respective mother-in-laws as being that of getting help in caring for their family. Caregiving by grandparents seems to be a normative process which reflects mutual reciprocity and obligation in relationships (Kataoka-Yahiro, Ceria, \& Yoder, 2004) as they provide support for immigrant-host families as they extend their duties in childcare for their grandchildren.

For the Apo family, both immigrant-host parents are working and cannot leave their only child at home alone. The family's maternal grandmother and the grandmother's sister were invited over to look after the immigrant-hosts' child. The guests therefore offered their time to take care of the child which also provides an opportunity to spend time with the grandson. Previously based in Wellington, it was also beneficial for the immigrant-host parents to bring their relatives as the husband found a job in Auckland which necessitated leaving his wife and son behind in Wellington for extended periods. Eventually, the entire Apo family decided to permanently move to Auckland:

To help us out while at the same time to experience living in another country. Immigrant-host daughter

Mainly to help us out with the child. We were having difficulties in sending my son to a day care for the last two months as he gets sick. We decided to bring relatives to help us look after [name of son] while we are at work. That's the main reason. The second reason was to have their company. Immigrant-host son-in-law

Because they wanted some company in their home. As well, I wanted to see my grandson. Visiting mother of immigrant-host daughter 
My niece invited me for a vacation so that I can at least see New Zealand [...] She was the one who paid for everything. I did not pay anything. I just need to go to her. Visiting auntie of immigrant-host daughter

Similar to Apo family, the Balbalasan family have two small children, one toddler and one infant. The visiting mother was invited to assist prior to her host daughter giving birth and to aid in post-pregnancy recovery:

First of all, I have job. Second, I just gave birth to my second child. My baby was only four months but I have to return to work. Immigrant-host daughter

Primarily, to take care of the kids. Immigrant-host son-in-law

I want to see my grandchildren and take care of them as I would pity them if they are just being left in a day care. Visiting mother of immigrant-host daughter

In contrast to the Apo and Balbalasan families, the Makiling family hosted their grandmother for the purpose of having her look after a toddler. Another motivation of the family is to emotionally recover after the visiting grandmother's husband passed away. In the narratives below, the motivation to host and the invitation to visit are expressed in more detail by the immigrant-host daughter and visiting mother than by the immigrant-host son-in-law:

Actually, my father died in 2011. I wanted my mother to recover from grieving by giving her a new environment. At the same time, I need her to look after my daughter and she doesn't get entertained back in the Philippines, she's alone at the house most of the time. [...] I just want her to unwind, change of environment, and at the same time, I need her to look after my daughter so that she gets amused since she is the only one left in the Philippines. Immigrant-host daughter

For her to travel and at the same time to look after my daughter. Immigranthost son-in-law

Because it was really depressing when my husband passed away. They encouraged me to visit and help them while at the same time to help me recover. So, I went to New Zealand and was somehow entertained because of my granddaughter. Then, my daughter did her very best from the very beginning, as we are extreme opposites. Visiting mother of immigrant-host daughter 


\subsubsection{Family visit}

A family visit was the primary motivation for VRs to visit their host relatives. What is common among the Arayat, Banahaw, Matutum, and Pulag families is that they do not have other relatives in New Zealand. As middle-class families in the Philippines, the relatives of the Banahaw and Pulag families paid for their own airfare to visit New Zealand while the airfare of the relatives of the Arayat and Matutum families were covered by their hosts. However, the meanings of their social interactions will be further explored in the latter sections of this chapter.

The entire Arayat family intended to visit the Philippines for Christmas. However, the immigrant-host granddaughter had decided to remain after finding work during the summer. The couple then decided to invite their guests so that their daughter would have company and not be left alone while they were away. While the decision to host was not for traditional child care as their daughter is of an age where she can work, the host couple was not willing to leave their daughter alone for an extended period while they were out of the country. The Arayat couple decided to make their overseas trip brief in order to return to New Zealand and host for their relatives. This intent was apparent within the following quotes of the immigrant-host father and the daughter while the immigrant-host mother provided other meanings in relation to her parents' visit:

Of course, to have time with my parents because they are very old. We only talk over the phone or through internet. There is a big difference when you see them physically. Then, we want to spend Christmas with them. They spent Christmas, New Year and my father also celebrated his birthday here. At least, we get to spend time with your parents. It's difficult to be here when your family is away. Spending time with them is priceless. Immigranthost daughter

To look after my daughter as we [I and my wife] are going to the Philippines and she will be left alone in the house. Immigrant-host son-in-law

Actually, they came here because my parents were going to the Philippines at the same time, and I got a scholarship at university, so, I can't go with them, so they came here so that they can look after me. My parents went to Philippines, so that is why they came. Immigrant-host granddaughter 
We are very happy to be invited and see what life they have which is progressive. Visiting father

We wanted to see their situation. With God's mercy, we saw that their family is happy and we are therefore contented. Visiting mother

The mother's visit to the Pulag family overlaps with the couple's plan to visit the Philippines. This is similar to the case of the Arayat family where the guest was invited to look after the children who will be left behind during the immigrant-host parents' absence. The visiting mother paid for her own trip to visit her grandchildren:

We hosted my mother because she wanted to see the children. It was her second time to visit us. The main reason is for them to see the kids because we cannot go home together being a big family. Immigrant-host daughter

Personally, my motivation is to be with them. Immigrant-host son-in-law

To be with them. Our grandparent also wants to see us. Immigrant-host grandson

I missed taking good care of them [grandchildren]. I wanted to experience that again. Visiting mother

Family reunion is the primary motivation for hosting of the Banahaw family. The visiting mother has not seen her only son for many years and she decided to visit the family at her own expense. The Banahaw family has not visited the Philippines for more than eight years making the visiting grandmother eager to visit the host family:

My mother was insisting that she wants to come, saying: "I wanna visit. I wanna see how you are doing there -- how settled you are." I think it was more for her peace of mind and I am her only son. Immigrant-host son

Because it has been awhile since I saw my mother-in-law. I really want her to see what is happening with the kids. Most of my children's cousins [in the paternal side] are in Bacolod or Manila but, at least they get to see each other compared to my children who don't have the opportunity to meet other relatives. Immigrant-host daughter-in-law

I miss him [my son], that's the main reason. Then, I want to see New Zealand. The last time I saw his family was in 2004. Visiting mother of immigrant-host son 
For the Matutum family, there is a consensus in terms of the motivations for the immigrant-host couple to invite their visitor. The family previously resided in the Middle East where the immigrant-host father was a migrant labourer. In this case, the immigrant-host children did not grow up in the Philippines and therefore did not experience living with their grandparents except when they returned to the Philippines for a brief visit. The grandchildren were fluent in speaking Tagalog as they were immersed in the Filipino community both in New Zealand and in their previous residence in the Middle East:

Because my parents are already old, I want them to experience how to travel. It's their first time to travel overseas. Then, I want them to see our family's situation and for them to experience the beauty of New Zealand. Immigrant-host daughter

Well, for me and my wife, it's our dream to bring them to New Zealand and show them our family's situation. We want to show them how blessed we are while sharing what we have right now. For industrious parents [in-laws] like them, they want to see that their children are fulfilled or blessed...not to show-off or be arrogant. Of course, we want to tour them here. Immigrant-host son-in-law

For our grandparents to experience travelling and ride an airplane. Also, we want them to show them what life we have here. Then, we want to spend longer time with them as I was only ten years old when we left the Philippines and moved to Saudi Arabia [before eventually migrating to New Zealand]. Immigrant-host granddaughter

To spend time with them because we have not seen them for so long -about five years. Immigrant-host grandson

First of all, we wanted to see how our daughter and her family have lived in a new country, where they work and where my grandchildren are currently studying. It feels good for a grandparent to visit where they have migrated. It was only a dream for me to travel overseas and I am thankful to God that it happened. Visiting father of immigrant-host daughter

We wanted to see our relatives' situation in New Zealand. And of course, we want to experience the life overseas. It was good seeing they are in good hands. Visiting mother of immigrant-host daughter

Within host-guest interactions, there was also difficulty in identifying the purpose of the visit as the individual responses of the Guiting-guiting family and their VRs were not uniform. While the nature of the visit was earlier classified under "family visit." the 
research participants have differing perspectives when it comes to their motivations for hosting. For instance, the immigrant-host daughter, her husband, and her visiting mother have differing opinions and responses and there are differences in the motivations of each family member, and categorising the social interaction as a "family visit" may not be sufficient to explain the phenomena given the familial dynamics of the visit. Meanwhile, the visiting sister was invited or sponsored by her brother and not by the Guiting-guiting family:

For my family to enjoy having a grandmother. We want our children to recognise someone who is elderly and learn how to respect them [..] For my sister, it was my brother who sponsored her trip. Immigrant-host daughter

I want to show to her that we are good here. Our situation is good and the country that we have chosen is really fantastic and I believe that she's convinced that New Zealand is the best place to live for-not only for myself but for our grandchildren and for her daughter-for their sister. Immigrant-host husband

My daughter invited me since she will enroll in a school [for her diploma]. I have to take care of my grandchildren. Visiting mother

Actually, it was not really them [Guiting-guiting family] who invited me, it was my youngest brother who migrated eventually after my sister's family. My younger brother was very close to me and treats me like a mother aside from being his sister. I am his confidante. He wanted to see me and for me to see the beautiful places that they experienced over there. It was our reunion. I decided to travel as our mother was also there during the time of my visit. So, I just satisfied my brother's long standing invitation. Visiting sister

\subsubsection{Eldercare}

For the Sierra Madre family, the visiting father is a widower having three daughters two of them reside in New Zealand while the other is in southern region of Luzon. Back in the Philippines, nearby immediate relatives regularly check on and look after the visiting father. While the stated principal motivation of the immigrant-host daughter of bringing her father to New Zealand is to ensure that he is well cared for, she and her visiting father actually demonstrate a relationship that exhibits interdependence and reciprocity which will be fleshed-out in section 5.3.2, care for others: 
Well, it's because of close family ties. No one will take care of him since he's too old. Then, two of us are now here in New Zealand [...] He is the only one left in our house [in the Philippines] although he has some nieces there; it is still different when it's the children who always look after their parents. I mean, to be with us. He's my only remaining parent. I want him to stay while he is still alive, with us. Immigrant-host daughter

To be with us. Immigrant-host son

I have to see my daughters and my grandchildren -- as well as my son-inlaw. Visiting father

\subsubsection{Celebration}

The Isarog family invited the parents and sister of the immigrant-host mother to become part of the silver wedding anniversary of the couple. With more than 400 invited guests attending the wedding anniversary, the immigrant-host daughter felt the need for her relatives in the Philippines to witness the celebration. Other members of the immigrant-host family have different motivations that are not as comprehensively articulated as the motivation expressed by the immigrant-host mother, who invited her parents and sister:

I actually made a promise to my dad two years before the wedding anniversary. He was very ill. I told him when I phoned that once he gets well, we will pay for their trip to New Zealand and that would be their fourth time coming here. For the past visits, it was just to visit us and the children but this time, they were really meant to come to attend our wedding anniversary. For my sister, it's another story because I gave her a part in the wedding like a bridesmaid -- but I think she wouldn't have come if not for my sister-in-law who told my brother that they would pay for her airfare. As I have already paid for my parents, my sister-in-law was happy to pay for my sister's airfare. Immigrant-host daughter

Well, it's some sort of sharing something what we have achieved here in New Zealand. Also at the same time, for them to witness the event [wedding]. Immigrant-host son-in-law

Well it was just you know that we wanted to show them that our life here in New Zealand -- and it's just probably the most important part for us seeing them after a very long time. Immigrant-host grandson $A$

To show them around. Immigrant-host grandson $B$ 
The host-family members often express differing motivations for hosting but, the respective VRs of the Isarog family have uniform responses when it comes to having similar motivations: experiencing and sharing in a family celebration. Filipino wedding anniversaries reflect the strong tradition of the family and involve many people in the ritual, particularly those who are significant to the celebrating couple:

I visited our relatives in New Zealand -- my grandchildren children, and my daughter because of the invitation of the $25^{\text {th }}$ wedding anniversary. Visiting father of immigrant-host daughter

She [my daughter] invited us to attend the $25^{\text {th }}$ wedding anniversary -- we feel a yearning to see them, we were forced to go [laughs]. In Filipino terms, you are longing for them since you have not seen them for many years. You feel excited to travel. Visiting mother of immigrant-host daughter

They invited us to attend the $25^{\text {th }}$ wedding anniversary. Visiting sister of immigrant-host daughter

\subsubsection{Assistance in finding employment}

Of the eleven immigrant-host family-VR social interactions examined, the relative of the Penablanca family was unique among the VRs that were examined in terms of motivation. The guest is a first-cousin of the mother of the immigrant-host husband. Therefore, she is referred to here as "visiting auntie of the Penablanca family." Aunties and uncles are terms usually used to refer to the biological siblings or half-siblings of one's parents. In the context of Filipino families, however, the classification of auntie or uncle is sometimes extended to cousins of one's parents (usually first cousins). However, sometimes more distant relatives of one's parents can be referred to as auntie or uncle (see Medina, 2001). Note earlier in Chapter Four that godparents are also referred to as auntie/uncle. After an early retirement in the Philippines, the visiting auntie has multiple motivations: travel and employment. However, a more altruistic motivation to host was expressed by the host family:

She is a member of the family -- blood is thicker than water. So, meaning, all family -- both sides, my side and my wife's side are welcome to our place. Immigrant-host nephew

It's just really to show this beautiful country. A lot of people are talking about the beauty of New Zealand. Also, we don't have many relatives here in New Zealand. So, sometimes when you have someone to visit here, it 
also feels good -- especially for my family, it's just the two of us [my husband] and our dog. If someone comes to you, you receive them. So basically, it's really to show this country. Immigrant-host wife

I was having a vacation in Australia. Then, I proceeded to New Zealand as I was hoping to find a prospective employer to sponsor me but I was already 55 which is the maximum age limit should one apply for a work permit. Unfortunately, I was not able to look for an employer although my visa was only good for two months. Then, I just reapplied for an extension so, I ended up staying there for nine months. Visiting auntie

With regard to expectations, most of the immigrant-host's(s') expectations towards the visit were mostly tied towards their motivations mentioned earlier and the enjoyment of the guest. On the other hand, the expectations of the VRs are tied towards fulfilling their obligation to the host family in New Zealand. Expectations are tentative representations of future events or unfinished learning processes which have a considerable amount of affect such as never before experienced encounters. It is determined by the person's felt needs and value system (Gnoth, 1997). Although most of the immigrant-hosts and VRs have prior interactions due to the frequent hosting/visiting and having known each other as relatives, one immigrant-host wife has not met her husband's auntie and has expressed her expectation which reflects a gendered dimension in hosting:

For me, I have not met Auntie before she came here. So, my expectation would be, I guess, is for her to feel comfortable since I am the female in the house. Therefore, I should look after the needs of our guest. Immigranthost wife, Penablanca family, group interview

As the majority of the VRs are return visitors to New Zealand as depicted in Table 5.1, the respective VRs did not express any prior expectation in relation to their visit other than fulfilling their social obligations with their host family. In contrast, the expectations of first-time visitors are not equally distributed as first-time visitors have expressed different responses, mainly: re-establishing ties, witnessing the living conditions of their relatives, quenching the longing for their family, seeing the New Zealand scenery, and finding employment. 
Conversely, anticipation and planning was mostly carried out by immigrant-host parents which becomes a marital decision when hosting and the task is assigned to the family member who is more knowledgeable with regard to filing an application to sponsor a relative to visit New Zealand. Back in the Philippines, it is usually the children or an immediate relative of the visiting parents or grandparents who are involved in obtaining documentation and in coordinating the trip of the VR(s).

\subsection{Social exchanges within the family}

This section addresses the second supplementary question: "What are the social exchanges between the immigrant-hosts and VRs?" Social interactions within VFR travel are multi-faceted and are composed of various social exchanges dealing with a person's significant other(s) such as relatives. However, studies on social exchange have overlooked work on intimate relationships (Collett, 2010) including those in tourism research. VFR travel as a social exchange deals with both the material and immaterial exchanges that are transacted between hosts and guests. When examining these phenomena, the social exchanges occurring such as hosting for or visiting relatives are intertwined with three recurring themes related to social exchange that emerged from the interviews and narratives of the research participants. These are the notions of: (a) interdependence, (b) caring for others and (c) familial obligations, are all interrelated in fulfilling of VR's duties with their respective host families and apparently the social exchange which can have a different meanings for the hosts (highlighted further in section 5.6).

\subsubsection{Interdependence of immigrant families and their visiting relatives}

Interdependence is a distinctive characteristic of social exchange which involves mutual and complementary arrangements (Cropanzano \& Mitchell, 2005; Molm, 1994). A social exchange perspective argues that family relationships become interdependent or interactional. It attends to norms of fairness and reciprocity, dynamics of dependence in relationships within families, and definitions of the rewards and costs associated with social exchanges in families (Chibucos, Leite, \& Weis, 2005). 
While there is less independent self-initiated mobility among VRs compared to VFs who travel within New Zealand, I argue that these VRs cannot be simply classified as wholly dependent guests and would best be described as interdependent guests. The VRs may not be able to travel elsewhere without some assistance due to reasons such as the lack of financial resources or health issues, but the reciprocity between the social interactions of VRs with their immigrant-hosts makes them interdependent. The immigrant-hosts and VRs fulfill their mutual social obligations in New Zealand (and even in the Philippines), whether it be by the VRs providing childcare, household assistance or emotional support to the immigrant-host family; the VRs are provided lodgings, meals, transportation and outings by the immigrant-hosts.

Identifying the five types of motivations earlier in sections 5.2.1 to 5.2.5, the narratives of immigrant-host families and VRs reflect interdependence. In the case of using kin for childcare accomplished by bringing VRs to New Zealand, immigrant-host families benefit as they recognise the importance of receiving a helping hand from family in providing childcare which is an advantage towards immigrant prosperity and professional integration.

Childcare arrangements are an essential element of parental employment (Uttal, 1999), particularly for the young immigrant families Apo, Balbalasan and Makiling as all the couples were working and trying to establish themselves in New Zealand. There are dynamics related to childcare and the interdependence between immigrant-host families (the host husband and wife) and their respective VRs. Underpinning the motivations of the immigrant-host husbands to host for their relatives, they recognise the significance of the benefit of childcare assistance provided by their respective relatives is (usually, by the female host's mother who has visited New Zealand more than once) in order to help them to handle their professional careers:

During the time that she visited, I was also busy so I wasn't able to give much of my time especially when I resigned from work and decided to start a business. However, I know that she thinks of us as good hosts. Immigranthost husband, Makiling family, individual interview 
I was relocated at that time and I wouldn't say there was a conflict because it became positive as if it was not for them, I wouldn't be able to take the job in Auckland as my family would have been left by themselves. At the time that I had to consider whether I would sign a contract, and if not for them [relatives], they [employer] might have given the job to another. [...] I don't think she could manage [by] herself with our son as she was also working. So, the visit did not create any conflict but was actually helpful. Immigrant-host husband, Apo family, individual interview

They know that we are very busy at work that is why we needed them to come here. Immigrant-host husband, Balbalasan family, individual interview

The immigrant-host daughter's social interactions reflect inter-generational care not only with her VR, but also with her family in the Philippines. Obligations towards grandparents and duties toward grandchildren are an extension of the parent-child relationship. Here, while Filipino immigrant-host mothers in New Zealand need the assistance of their immediate relatives to take care of a child, they also have the obligation to occasionally look after their parents or probably other immediate members of the family in the Philippines. Visiting relatives are also making sacrifices by being in New Zealand where they are faced with physical difficulties due to their age:

My obligation to her is give her an allowance so that she benefits rather than for me to give it to someone else such as the day care. I'd rather give to her so she has income when she goes home, or if she needs to buy something else for her parents since my grandparents are still alive. She sends money to her parents. Immigrant-host wife, individual interview Balbalasan family

They have a big responsibility in me. When I got sick [while in New Zealand], one of them filed a leave of absence. As a couple, they are kind to me. Visiting mother for Balbalasan family

Child care then becomes a social exchange which demands mutual trust between the parents and the caregiver and is embedded in the socio-cultural beliefs of a family (which will be further explained in section 5.5 related to the caregiving practices of Filipinos). Apparently, the act of caregiving expressed by the VR is extended as their contribution to economic resources are also shared by their 
families in the Philippines who are making sacrifices while the VR is away and visiting long-term in New Zealand; this has a significant impact on the family dynamic back in the Philippines. The immigrant-hosts in turn benefit from having their children under care of a VR which allows host-mothers to become productive in the economic sphere and provide the host family more financial capacity to provide economic support to their families in the Philippines through remittances, at the expense or cost in the time and effort of a visiting relative from the Philippines. Thus, the caregiving provided by the VRs is a form of symbiosis that benefits both families in New Zealand and back home in the Philippines:

I enjoyed having less household work. At least, when I came home, the food is prepared. My husband and I were not stressed. Also, my son did not need to wake up very early to go to the day care. With his stature, he is very thin and needs more attention. My auntie [a public school teacher] taught my son how to write. My son became more fluent in Tagalog when they were here. Immigrant-host daughter, Apo family, individual interview

My niece is really kind. So, when she says to me, "Auntie, are you still coming back? We need you here." I tell her, "Yes, I'm coming back." As for my mother [the host daughter's grandmother], she [the host daughter] provides her medicine. Imagine [that]?! She's very kind. Where can you find a granddaughter like that considering we, the children [of the grandmother] are here [with her in the Philippines]? She sends money weekly for her medication [tears flowing]. Visiting auntie of Apo family, individual interview

\subsubsection{Caring for others}

The social exchanges between host(s) and guest(s) are engaged by way of taking care of one another. This may mean that Filipino VRs to New Zealand bear the costs for their own travel, particularly for middle-class grandparents, in order to spend time with their relatives and typically occurs when newer immigrant-host families cannot afford to pay for their VR's trip. Unlike the immigrant-hosts and VFs social interaction discussed earlier, social exchanges are also extended in the post-visit phase as immigrant-host children have a duty or obligation to keep in touch with their relatives (especially grandparents) in their family's former homeland. 
Earlier, we have learned that VRs provide help with child care and how this assistance is a significant benefit both to the families in New Zealand and the Philippines. There is also a mutual relationship between immigrant-hosts and VRs as care is provided during difficult times. Child care provided by a trusted and loving caregiver is crucial to families who work long hours to make the incomes required to adequately support their family in a developed country such as New Zealand. Nonetheless, immigrant-hosts reciprocate their VRs' sacrifices by diligently looking after their wellbeing in exchange for child care. While the visiting mother for the Makiling family was struggling with the loss of her husband, she had decided to assist her daughter, who is working as a nurse, and the VR handled all the household responsibilities. The host family and their guest provide this perspective about childcare:

Even if my mother does look after the household chores, I still check things with her and provide her the necessities. At the same time, I am a family member as I should look after her wellbeing. [...] My mother has her own initiative of doing things. You can't ask for anything from my mother because even if you don't require her to do something in the house or take care of children and she does it naturally. Immigrant-host daughter, Makiling family, individual interview

She is not really required to take care of the house. It's just for my daughter to have a companion at home. Mommy is a bit old and she may not be able to handle everything. Immigrant-host son-in-law, Makiling family, individual interview

I want my daughter to go home seeing that the house is clean and that the clothes have been folded. Sometimes she tells me not to do the laundry but I am still doing it because I want to see that she is happy. Visiting mother for Makiling family

Even if the Guiting-guiting family's children are in the primary and secondary school, the parents cannot leave their children alone after school, yet they also need to work. On the other hand, it was clear to the visiting grandmother that her duty is to look after her grandchildren while, the visiting sister was also well cared for by her brother yet, she does not have any actual obligations even though she was visiting the Guitingguiting family and sleeps with her nieces: 
When my mother visited, I was busy studying in for three months full time. And then, on the last stage of my studies, I had an internship in Auckland Hospital. So, that's also one of the reasons why we need her to come here as I will be on duty for work. Immigrant-host daughter, individual interview

We provided all the comforts that we can afford, our time -- despite being busy at work -- because you know, a host should attend to their visitors. Immigrant-host son-in-law, group interview

I am their companion at home. I sometimes cook Bicolano dishes ${ }^{11}$. My daughter wakes up early and cooks in the morning as I am still sleeping. There is no conflict in household work. I was never pressured actually. If I want to do the vacuum, my son-in-law resists. If I want to do the laundry, my daughter doesn't want. What will I do here? Instead, I just do some gardening. Visiting mother

Just to make sure that I relax, that's it. They did not impose any obligation for my visit. Visiting sister

The Pulag family has four children and as a result they cannot afford to travel all together to the Philippines. So, they decided to have their visiting mother look after the children while they are travelling. As well, their older son has moved out of the house at the time of their guest's visit so, good solution to their child care issues was to invite family from their homeland to assist. The visiting mother paid for her own trip to New Zealand as she is eager to spend time with her grandchildren as she does not have any in the Philippines:

I managed the house and I hope that my daughter appreciates it. I did everything while they were away as if the parents [of the children] were there. Visiting (grand)mother

There is also a conscious effort to maintain the transnational familial relationship between grandparents with the immigrant-host children as reflected in the previous trip to New Zealand made by the grandparents. As much as possible, the grandparents for the Pulag family make sure to visit New Zealand in order to continue their ties with their grandchildren as they also had the opportunity to take care of them in the Philippines before the family migrated. Even after the visits, the grandparents are eager

\footnotetext{
${ }^{11}$ The Bicolano cuisine is popular for the use of coconut milk and chili pepper.
} 
to continue to maintain their ties with their grandchildren and vice versa as both sides make an effort to regularly communicate:

I have to remind my siblings to make sure that they also get in touch with my grandparents. For me, I can do it by sending them an e-mail message at my own. However, my grandparents also would like to know how my siblings are doing. During my grandfather's birthday, I also sent a gift to him. Immigrant-host son/grandson, group interview

We talk to our grandchildren over the internet. It has been our tradition that when someone celebrated his or her birthday, I buy a cake from Red Ribbon ${ }^{12}$. Then, they blow the candle [virtually]. Visiting (grand)mother

In order to determine the wellbeing of the immigrant-host family in New Zealand, another (grand)mother paid for her own trip to visit her son (immigrant-host husband) and the rest the Banahaw family as they have not seen each other for more than eight years. The visiting (grand)mother was very concerned with the family's living situation after her daughter-in-law had been diagnosed with cancer and had to undergo a series of therapies which became an impediment for Banahaw family to visit the Philippines. The visiting (grand)mother also greatly missed the immigrant-host husband, who is her only son and this was a major motivation for her travel. While the visiting (grand)mother was not expressly invited to come to New Zealand, the son nevertheless felt an obligation to reciprocate his mother's efforts to visit by hosting her very well. While the immigrant-host husband does not communicate with his mother frequently, the immigrant-host daughter-in-law feels the need to show her concern and respect and keeps in touch with her mother-in-law:

Because the wife is sick and they have many financial obligations and that's the first reason why I visited them to see their situation there. [...] While I was there, I tried to spend my own money because I don't like my children to spend for me. When we go out sometimes, I pay for the expenses. The first reason why I went there is to see my son. Visiting (grand)mother for Banahaw family

You have to take care of her all the time. You have to drive for her. My mom won't take the bus. You know, I'm busy at work. When we go to work, she stay here and watch TV. She'll fold the laundry and stuff like that. When we go home in the afternoon, we sit at the couch together chatting, laughing, eating. Immigrant-host son, Banahaw family, individual interview

\footnotetext{
${ }^{12}$ A popular chain of pastry shops in the Philippines.
} 
It's not an obligation [to keep in touch] -- but you make it a point that no matter how far you are, you let her feel important by contacting her. Immigrant-host daughter-in-law, Banahaw family individual interview

Earlier, we saw the motivation of the host-daughter for Sierra Madre (a solo parent) to look after her father who is a widower. She is also concerned as her father is now living without immediate family nearby (but with some extended family members) in a remote province in the Philippines. Of the three daughters of the visiting father, two of his children are now living in New Zealand (both of them are nurses and one is married to a New Zealander). The remaining sibling of the immigrant-host daughter is based south of Manila which is very far from their former hometown, where their father still lives. Initially during his visit, the visiting father was accommodated by his daughter (who is married to a New Zealander and has children) until the host-daughter for Sierra Madre bought a house. A licensed engineer by profession who is probably in his $80 \mathrm{~s}$, the visiting father managed to perform some maintenance on his daughter's home. She greatly benefitted from this assistance as she has a very erratic schedule being a nurse and does not have the time or knowledge to perform home renovation tasks herself. Knowing that his host daughter is a solo parent, the visiting father also taught his grandson to drive a car, which is almost a necessity when living in New Zealand:

I am happy because at least he witnessed my accomplishment and I think he was also happy arranging some parts of the house. [...] He did many things for the house which is very risky and I reminded him about that. [...] I felt that this is like his home. Actually, we have viewed many houses before buying this house. In most of the houses that we've seen, he feels negative. And this house, this was his choice. Immigrant-host daughter, Sierra Madre family, individual interview

When she bought the house, I was also the one doing the gardening even during the winter. I need it for physical activity especially if you're not doing anything. When my daughter is on duty [as a nurse at the hospital], my grandson and I fetch him to the train station. Visiting (grand)father for Sierra Madre family 


\subsubsection{Familial obligation}

The social obligation of hosting for relatives is really a reciprocal relationship as the guest/s may need to travel to fulfil their familial duties. Other than taking care of children and helping the immigrant-host family, there are familial obligations which would be similar to the interdependence and the mutual concern for relatives (identified earlier in sections 5.3.1 and 5.3.2) that require face-to-face interactions such as celebrating a special occasion or when immigrant families express their gratefulness to their parents by showing them their new homeland which is an inherent cultural norm of Filipino children who were raised in the Philippines (which will be elaborated on further in section 5.5). Other than hosting for immediate relatives, an immigranthost family may also accommodate distant relatives as a familial obligation.

As for the Isarog family, while the guests (parents and sister) were invited to celebrate the silver wedding anniversary, the parents maintained their ordinary lives as if they were back the Philippines and involved themselves in the domestic sphere of the immigrant-host family. The immigrant-hosts and VRs have provided different responses with regard to their obligations to one another while the immigrant-host grandsons cannot identify any of their grandparents' obligations while they were in New Zealand:

My father gets to fix the stuff in the garage, old items so that's what he does every time he comes here. He gets rid of the clutter and then he makes some tables or chairs out of that. Immigrant-host daughter, Isarog family, individual interview

From my own perspective I think really it's just to be there, to support and to be there as parents for the occasion -- special occasion -- to catch up with my kids. Immigrant-host son-in-law, Isarog family, individual interview

Every time we are there -- because it's our fourth time, I try to maintain their house when I'm there. Anything that deals with house repair, I do that. For my wife, she does the cooking and laundry. Visiting (grand)father for Isarog family, group interview

On her wedding day, I made sure that her gown fits well and looks nice on her. But during ordinary days, I just cook for them even if my grandson 
knows how to cook [being a chef]. Visiting (grand)mother for Isarog family, group interview

A similar response was elicited from the immigrant-host granddaughter of the Arayat family regarding her grandparents' obligations while they were staying with them. As indicated in the earlier section, the parents for the Arayat family had planned to return to the Philippines for a brief visit but were concerned about leaving behind their daughter who had to do a paid internship with the university. Hence, they thought of inviting their relatives to stay in their home so that the granddaughter has companions at home. While the grandparents mainly stay at home due to their physical limitations and unfamiliarity with the area, the fact that they are in another place (country) provided a notion for the granddaughter that her grandparents were on a holiday:

My grandmother did quite a bit of cooking because she likes to cook and she also likes to do the laundry and stuff-she did quite a bit of laundry just to pass time because they can't really go out-she doesn't really walk around a lot. It was just quite a holiday for them, and that is all that we wanted. Immigrant-host granddaughter, Arayat family, group interview

Likewise, the Matutum family invited and brought their relatives to New Zealand for a visit. While the family is not affluent, the host-couple were inspired to bring their relatives to New Zealand based on the experiences of other Filipino families. Unlike other immigrant families who have children at home that need supervision, the guests had no child rearing obligations within their hosting household because the children were almost fully grown:

We [together with husband] saved money and also with a loan from the bank. We tried to find a way to raise funds because a lot of our friends told me that while one's parents are still healthy, let them come to New Zealand. Like them, they were very sorry that they did not have the chance to bring them here until they got sick or eventually passed away. Immigrant-host mother, Matutum family, group interview

For the Penablanca family, they accommodated their guest as she was initially looking for employment. Despite being a distant relative, the reciprocity is reflected by maintaining a smooth interpersonal relationship within the household: 
We did not impose any obligations. Since she is part of our family, she just needed to act normally in the house. Sometimes she washed the dishes or cooked rice. [...] It's not really a responsibility. It's like she's in her own home. Immigrant-host wife, Penablanca family, group interview

The couple hosted for me without asking anything in return...monetary or whatsoever, just because I'm the auntie of [immigrant-host nephew], I am very much welcome. Visiting auntie of Penablanca family

Overall, as the immigrant-host's(s') and VR's(s') social interactions were discussed in relation to the purpose of the visit, motivations, and expectations, the nature of the social exchange between the immigrant-hosts and VRs are oriented towards maintaining family relationships. As this section has examined the social exchanges which occurred between immigrant-hosts and VRs which primarily occurs due to interdependence, care for others, and familial obligation, the discourse on social relationships in the family has neglected the emotional dimensions of family life because emotion has often been considered "too personal" to openly discuss and is treated as if it has an existence independent of the social and cultural context (e.g., Parreñas, 2001). The next section will deal with the nature of emotional solidarity between hosts and guests.

\subsection{Familial solidarity: social identities and shared social interactions}

In this section, I address the third supplementary research question: "How do social interactions reflect emotional solidarity between the immigrant-hosts and VRs as expressed by their shared beliefs and behaviours?" To illustrate how social interactions are reproduced through the emotional solidarity between immigrant-host families and VRs, their social identity whether as host/guest or as relative is presented in relation to their significant others (section 5.4.1). In this case, emotional solidarity is defined as: "feeling a sense of identification with others, or the identifying with other individuals as a result of a common value system" (Wallace \& Wolf, 2006; Woosnam et al., 2009). The memorable aspect of the visit was also captured within section 5.4.2 for each pair of immigrant-hosts and VRs and shows how "ordinary" experiences become "extraordinary" when shared by them. 


\subsubsection{Hospitality and sociability between immigrant-hosts and VRs}

While hospitality is often viewed in relation to "others", Snow (2010) also considered it as an act of benevolence or virtue in making people feel welcome in a "shared life" and world. In this case, it symbolizes the "being together" in New Zealand of the immigrant-hosts and their visiting relatives from the Philippines. This reveals how immigrant-host families share their world in New Zealand with their relatives from the Philippines and how VRs become part of the everyday life that immigrant-host families perform in their receiving country.

Using the theory of emotional solidarity as the conceptual lens, immigrant-host family members and VRs provided their views as to whether they see themselves as hosts or guests, respectively, in relation to their social interactions. This approach is able to illuminate the emotional solidarity between and amongst the hosts and VRs by examining such through their social identity as relatives, where emotions are more evident between and among relatives regardless of the temporal dimensions of their social interactions (pre-, during-, and post-visit). The presentation of this sub-section begins by examining the perspectives of the hosts - those of the immigrant-host mothers, fathers, and children, followed by the perspectives of the VRs, who are mostly grandparents to the immigrant-host children in New Zealand. For immigrant-host parents/couples in New Zealand, the expression of their social identities was very prevalent such that the majority of immigrant-host mothers perceived themselves to be daughters as well as hosts for their relatives. The perspectives of the immigranthost mothers are first examined due to the significant bond that exists between them and their guests. As daughters, they maintain the traditional role of Filipino children who look after their parents, who previously nurtured them during their childhood in the Philippines. Hence, reciprocity is clearly articulated via the immigrant-host mothers' social interactions with their Filipino parents:

I am still his daughter actually. I was the one providing -- it's really like -- I want him to be settled -- I mean happy that he doesn't have to think of anything. [...] I am still his daughter, and he is still my father. He is very helpful. You will really feel that even if he is already old, he is still important. That's like how he wants us to feel that he is important and helpful. Immigrant-host mother, Sierra Madre family, individual interview 
I feel that I am still her daughter around the house. I leave everything to her such as buying groceries. The supermarket is walking distance. Her role as mother 20 years ago is being done again. Immigrant-host mother, Balbalasan family, individual interview

As a daughter who is working/living in another country, your longing for your parents becomes deeper. All the good deeds that you do, you show your love which is immeasurable because I cannot repay the love that she gave me. That's why as much as possible, I will give everything, even if I cannot surpass what she has given me so, that she can be happy for me as her daughter. Immigrant-host mother, Makiling family, individual interview

However, some immigrant-host mothers also view themselves as hosts in relation to their position or status as residents in New Zealand, since their relatives are not familiar with the place. Therefore, they need to provide for or attend to the material needs of their guests:

A host. You provide their food especially as they have special diet since both of them have high blood [pressure]. So, you have to have vegetables and fish. Then, you want to make them comfortable so you give them blankets -- those basic needs they need to survive the winter. [...] They are visitors and they don't know much about here. Immigrant-host mother, Apo family, individual interview

I think it is being a proper host, you know. You find means for them to enjoy their stay. You are a tour guide. You also tell them what should be done in an emergency. Immigrant-host mother, Pulag family, individual interview

Some immigrant-host mothers also define their hosting in relation to their hospitality and cultural obligations to their relatives even while in New Zealand. Hosting a family member is an expression of their respect by being able to serve and care for them and ensure that their relatives profit the most from their visit in New Zealand:

Because traditionally in our culture, we are hospitable people and it is respectful to your family and it's an obligation. Immigrant-host mother, Isarog family, individual interview

As a host, you have to entertain them very well -- you have to make them [parents] happy. You make the most out of their stay. Immigrant-host daughter, Matutum family, individual interview

Since hosting for family members is almost an obligation, other immigrant-host mothers also express a guilty feeling if they were not able to offer much of their time 
during the visit of their relatives. In this case, time becomes an essential element that has to be shared with their relatives despite the fact that immigrant-host mothers are burdened with work within and outside of the home:

Well, I was always a host [to everyone], although I cannot say whether I was an excellent host, because I was not able to give them much time as much as I wanted too. Immigrant-host mother, Guiting-guiting family, individual interview

[Both] as a daughter or as a host. However, you also feel hesitant being a host because you have to work. You have to consider that your [parents] will be alright while you are away and that everything they need is here. Immigrant-host mother, Arayat family, individual interview

With regard to the immigrant-host wife's relationship with her mother-in-law, taking care of the guest and providing her time to her relative even when they cannot go out on a tour is important:

I would consider myself to be a host because we try to look after her, most of the time. We don't leave her on her own. For us, if we cannot tour her, we'd rather stay at home and have a quiet dinner or lunch together. Immigrant-host daughter-in-law, Banahaw family, individual interview

In contrast, being an active member within the Filipino community, the Penablanca immigrant-host wife's view of hosting is tied toward introducing her husband's visiting auntie to other Filipinos and bringing the guest to several socio-civic activities in which she is involved:

I see myself as a host. Being a host is not just accommodating or feeding them in your own home. I think hosting is about extending your network to her and making sure that her visit is purposeful. Immigrant-host wife, individual interview

Consequently, immigrant-host fathers (or the sons-in-law of most of the VRs) believe that they are hosts based on the material resources that they have to share or contribute within the household. In the context of the Filipino culture, being a good father means primarily providing for the family as they are figuratively called haliging tahanan which means the cornerstone of the home (Harper, 2010; McKay, 2011; Rubio \& Green, 2009). The hosting of relatives by the immigrant-host fathers through sharing 
the family's material goods produces positive emotions, thereby reinforcing their emotional solidarity and identity as hosts:

I am a host. Of course as the father, you provide for all their needs. Immigrant-host son-in-law, Matutum family, individual interview

Yes, I am a host because I was able to provide whatever they needed such as accommodations and food. Immigrant-host son-in-law, Apo family individual interview

In our experience as a host, we saw that they enjoyed it. We are happy to share what we have here. We have a house, car, but more than that, we enjoyed going out to see what we have here in Wellington. Immigrant-host son-in-law, Pulag family, individual interview

While immigrant-host fathers express their feelings of being a host to their VRs (mostly with their in-laws), they need to maintain their usual routine in order to properly provide for their family. Similar to the responses of immigrant-host mothers earlier, time spent with the visitors was also an important aspect of hosting for fathers:

What should [I say] -- Normal. Just like a companion at home. I am still a host because you tour them around when you have a time. Of course, you also need to work. If you have time, you go out. Immigrant-host son-in-law, Balbalasan family, individual interview

An immigrant-host husband may also express his esteem with regard to hosting his mother-in-law:

I am a host -- but, she's [mother-in-law] not just an ordinary visitor. I must see to it that she would feel that she is valued and welcome in our home. Immigrant-host son-in-law, Guiting-guiting family, individual interview

Hospitality can also elicit a sense of pride for an immigrant-host son as his siblings and other relatives in the Philippines learn about his hosting of his mother:

[I am a host] because she tells everybody else at home at that she had fun and she was well cared for. Immigrant-host son, Banahaw family, individual interview

In contrast, the immigrant-host nephew for the Penablanca family perceives his identity in relation to his auntie as a relative and not as a host. As the Filipino family is 
described as traditionally consanguineal, blood ties are considered so important that even relationships with distant cousins, aunties, and uncles are recognised:

I don't see myself as a host because auntie is a member of the family. Our house is open to everyone, that's how we are as a couple. Immigrant-host nephew, Penablanca family, individual interview

Meanwhile, depending on the value orientation and situation of the immigrant-host children, whether they were raised by their immediate relatives in the Philippines or had grown up in New Zealand, they have different interpretations about themselves. For example, since a significant bond still remains between the immigrant-host child and the relatives who took care of him as a grandchild, he does not feel like he was a host:

I see myself as a family member -- I don't see myself as a host or something because we [he and his grandfather] are always together. Immigrant-host grandson, Sierra Madre family, individual interview

Immigrant-host children may also view hosting as an opportunity to become a companion for their VRs (grandparents) who may not be familiar with New Zealand. Hence, being accommodated in the family home, these children still view themselves as hosts of their relatives:

I see myself as a host because we have accommodated them here and we toured them around. Our interaction was important as we spent time together. Immigrant-host grandson, Pulag family, individual interview

Maybe I am a host because if you have a visitor, you have to entertain and tour them. Immigrant-host granddaughter, Matutum family, individual interview

I am host because they are not familiar with the place and you show and tell them everything about New Zealand. Immigrant-host grandson, Matutum family, individual interview

I perceive myself as host. I have to keep them company. I think it was quite a good experience for them to come here and-because in the Philippines, they have work's principles so, they do house work. And I just thought, it was good for them to have a holiday. Immigrant-host granddaughter, Arayat family, individual interview

In the context of domestic hospitality, hosting is an offering of the gift of one's time to cultivate ties (Russo, 2014). Apparently, the amount of time spent with their guest(s) 
is also an essential element in hosting for immigrant-host children who grew up in New Zealand. This may be similar to the findings of Schänzel et al. (2014) where younger generations who were born (or raised) in New Zealand display more self-orientated and individualistic tendencies compared with their parents when hosting for their VFRs (further explored in section 5.5.4: Barriers to familial solidarity):

Not really. It's a [big] responsibility as a host -- but if I was a host though, I would -- you know really take them around but, it's just that, the time, you know? That's why there are more family members to help you do stuff. I got my own stuff nowadays, you know. I'm busy, you know they came when I had no time. Immigrant-host grandson A, Isarog family, individual interview

I think I would make a good host, although at that time it was quite difficult because, like I said exams, and in fact I felt very guilty just sitting in my room, you know studying. Immigrant-host grandson B, Isarog family, individual interview

In contrast, evidence from most of the responses from VRs was uniform as expressed in their social identity as relatives rather than as guests. Therefore, the VRs' identities are shaped in relation to their being part of the family. The succeeding narratives will show how intimate relationships are expressed, not only through the shared beliefs and behaviours identified in the theory of emotional solidarity, but also through "shared identities" where these guests express feelings of "being at one" or "in union" with their host families, or an inter-relationship that is based on pakikipagpalagayangloob or level of mutual trust. In this case, the interpretation of the "self" for VRs reflects their traditional roles as Filipino parents/grandparents insomuch as assisting their children in New Zealand in terms of domestic responsibilities. In the context of VFR travel, there is a need to inquire about how particular roles and relationships are engendered and examine what people mean by the emotions that they evoke and intimacy that they demonstrate. As such, even when a traveller leaves home, home does not leave the traveller (Duncan \& Lambert, 2003; Germann Molz, 2005; Larsen, 2008; Pons, 2003) and this is very much observed by the accounts provided by the VRs:

I am part of the family. They are my children. Visiting father for Sierra Madre family

They are my children and I feel at home [...] Of course, it includes my sonin-law. I don't treat him like others. I feel that he is my son, so, I don't feel uncomfortable. When I was there, I feel that I am in my own home. I make 
decisions even for the groceries. Visiting mother for Balbalasan family

No, I am not a guest. They treated me like a father and they are my children. Visiting father for Arayat family

I feel like a parent and they make me feel [that way, or should I say] -- a grandparent to our grandchildren. You can see how the family welcomed me while I was there. Visiting mother, Pulag family

My visit was to serve my children while I was there. Right now, I cannot serve them because I am far away. I have done everything to fulfill the responsibilities of a parent. Visiting mother for Arayat family

I am part of the family. I make sure that I was helping with all their house work because I know they are working and very tired. So, they have no time to do the house work. Visiting mother for Banahaw family

It's like we are in our own home although it's a different setting. You are not being treated as a guest. You act as a normal resident in the house. We don't ask our children to look after us. We don't act like a VIP's [very important person]. Visiting mother for Guiting-guiting family

Other VRs also felt in solidarity by having a sense of being a relative rather than as a guest of their host/s. Even when they are overseas, guests may still feel "at home" with their relatives accommodating them in New Zealand:

We are part of the family. We are not a visitor. When we are there, we do household chores. I told my daughter, "Just get ready for your work and we'll take care of things here." We are part of the family, not a visitor although we are holding a visitor visa [giggles]. Visiting father for Isarog family

My role is being a mother. I am very proud of her especially during the $25^{\text {th }}$ wedding anniversary. We were there as her parents -- we were the last to march to the altar. Visiting mother for Isarog family

The place may be strange. I don't know where to go as it was my first time to visit. However, I saw myself as a family member. Visiting sister for Isarog family

In comparison, some VRs may feel like a guest (or an "outsider") within the house since the immigrant-host family makes decisions about the household management, and all the while they try contributing in the household responsibilities in order to maintain smooth interpersonal relationship(s) with their host(s) or, as reflected in their 
pakikibagay, by conforming to the host family:

Of course, we are visitors. They gave what we needed. Then, we shopped during the weekend [...] I feel I am a visitor because they are the ones who own the house. Visiting mother for Apo family, individual interview

Like I am part of the family -- but you also have to work. You can't just lie down or relax; or I would feel uncomfortable. After all, I don't feel like being lazy. You want to perspire since it's very cold there. It feels good to work. Visiting auntie for Apo family, individual interview

Maybe -- but I am not the kind of person who will just make decision in their house, like "what are we going to cook", even if they told me to. I can do what I want in their place but, I still respect my children. Visiting mother for Makiling family

Guests may also acknowledge the hospitality and the way that they were treated by their relatives and/or included in the lives of their host(s) while in New Zealand. A visiting relative may become engaged in the usual routine chores or activities of their host:

More than a guest -- that's how I was treated. My sadness was gone while I was there. My nieces were very happy that I was there. Visiting sister for Guiting-guiting family

I was a guest because they treated me especially. They didn't let me do the heavy work at home although, I assist my nephew during winter; like picking small pieces of wood [used for fire] which he gathered at the backyard. Also, I helped her [immigrant-host wife] in the Rugby World Cup as the Filipino community prepared for an ati-atihan ${ }^{13}$ performance. Visiting auntie for Penablanca family

Clearly, the immigrant-hosts and the VRs provided different interpretations respectively with regard to their being a host or guest. A range of answers were presented earlier as research participants provided their responses. The self is relational - often entangled with significant others and this has implications for self-definition, self-evaluation, selfregulation and most broadly for personality functioning expressed in relation to others (Anderson \& Chen, 2002). Both hosting and visiting are social obligations that require the co-presence of family members. Through the theory of emotional solidarity,

\footnotetext{
${ }^{13}$ The festival consists of tribal dance, music, accompanied by indigenous costumes and weapons, and parade along the street.
} 
hospitality and sociability between immigrant-hosts and VRs are illustrated by the shared identities that they express as family members. Whether immigrant-host families identify themselves as hosts or as relatives, time is an essential component in hosting as one's significant other (the VR) offers themselves whether to help the household, to celebrate an important occasion or milestone or, to renew their ties - all of which is an expression of how much one is being valued as a family member within a New Zealand household. The next section deals with the experiences that are shared by the immigrant-hosts and VRs through their social interactions.

\subsubsection{Extraordinariness of everydayness}

The theory of emotional solidarity also reveals the affective dimensions of being together for the immigrant-hosts and VRs as they share the memorable aspects of their visit. What also becomes special for the families seem to be the ordinary moments between their major interactions. Shani (2013, p. 11-12) argues that the conceptualisations of tourism in general, and of VFR travel in particular, should capture the "everydayness" elements of travelling without neglecting the "extraordinary" components that are still present in the tourism experience. As the emotional solidarity between the immigrant-hosts and VRs are analyzed by examining both individual and family interviews conducted after the visit, research participants seem to provide somewhat uniform responses that are starkly focused on the ordinary and routine.

Even if the social interactions of the immigrant-hosts and VRs were examined across the temporal and spatial dimensions, they still cite co-presence as being remarkable and it is that which cements their relationship. In this case, the exchanges made through the visits suggest that emotions are the primary foundation for group formation (Lawler, Thye, \& Yoon, 2000), particularly when relatives are together making the social interactions that become beneficial for both hosts and for the visiting family members. When presenting the results of the analysis, an attempt was made to examine the emotional solidarity between one select family unit vis-à-vis their guest/s as each social interaction was unique to and specific to an individual immigrant-host family member and a respective visiting relative in relation to the memorable aspects of "hosting" or "visiting" New Zealand. 
Being a religious family, the host daughter for the Sierra Madre family remembers her time with her father who accompanied the rest of the family on an outing to church on a Sunday, while the host grandson had enjoyed the previous trip they had as a family (together with his auntie's family) to Auckland. As the grandfather cannot recall the name of the places that they had previously visited, what he did consider as being memorable was simply being with the Sierra Madre family and with his other daughter's family who live in New Zealand:

When he goes to the [Catholic] fellowship, there are instances that I cannot attend, but he goes there with my son. Immigrant-host daughter

When we went to the amusement park [Rainbow's End], even if he did not go on the rides, I saw how happy he was for us and especially for his grandchildren who were having fun. Immigrant-host grandson

Now that I'm back, I can only remember my children and grandchildren. Visiting father

The previous inability of the Banahaw family to travel back to the Philippines and after many years makes it all the more memorable for the couple to host their grandmother in New Zealand and to at the very least, take her to a nearby destination. Being the only son who is no longer with her in the Philippines, the visiting grandmother enjoys spending any time spent with him whenever they go out. However, she cannot remember the places that she visited despite the researcher showing some photos that the Banahaw family had provided:

Maybe, when we went to Staglands [Upper Hutt] together with our friends. My mother-in-law really enjoyed that event. Immigrant-host daughter-inlaw

Seeing my mother again and we'd go out. We'd drive and walk together holding hands. Immigrant-host son

You know when I was there, every morning, my son would take me out when he had no work; just the two of us. Then, he showed me some places that I can't remember [laughing]. Visiting mother

The commensality of food makes the visit memorable for the daughter of the Makiling family (together with her other sister who has a family) and is mutually felt by the 
visiting mother. Special occasions such as Christmas, which the visiting mother celebrated for the first time without her recently deceased husband, become significant. For the host husband, ordinary days or bringing the whole family for a trip outside Auckland makes the visit extraordinary:

Having a meal or when we'd go to my sister's place to chat or for deeper conversation. That's the best time for me. Immigrant-host daughter

It's the driving. Well, I think almost everything - like when we went to Taupo or even when we were just at home. Immigrant-host son-in-law

When I was with them during Christmas, I could really feel their love. During normal days, when we'd have a dinner, my daughter would put rice on my plate because that's what my [late] husband used to do for me. Visiting mother

Similar to the earlier case, being together in the presence of food, or together while dining, makes the social interactions memorable for the Arayat family and the host mother also very much enjoyed these times with her visiting parents. In the context of shared experiences, immigrant-hosts most frequently cite memories involving copresence, such as a family dinner. This indicates that they may be longing for the time with their family and comfort in being with others with whom they share commonalities, not only with others that share their native culture and ethnicity, but also their shared identity as relatives. Whereas VRs (from the host's(s') former homeland) would usually have more opportunity for social situations with family, as family is usually more abundant if one does not move away from other members within their kinship circle (assuming the VRs are still with other members of the extended family in the Philippines, as compared with immigrant-host families where the structure becomes nuclear after migration). Therefore a VR may not feel as great a longing for, or cite significant memories of co-presence and they may have more significant memories regarding events or places, like going out to a mall or park which occurs because of their hosts:

Everything, I think. Immigrant-host daughter

I think it's going out even when we are just going to a restaurant. Immigranthost son-in-law

Probably, just like when we're all here with my parents and when we would 
go have like dinner and stuff because it was just all of us, like talking and just spending time with each other, that was probably the best. Immigrant-host granddaughter

While I was there, I saw Jose Mari Chan ${ }^{14}$ whom I never saw in the Philippines. He did a concert there. Visiting father

One thing I remember is going to the malls and buying some stuff, like food. Then, we also went to her [my daughter's] friend's [place]. There are so many things you cannot forget. I know that her friends there are very kind because she is like me and knows how to deal with others. When she was invited by her friends to celebrate birthdays, she brought us there. I was really happy. Visiting mother

In this instance, making an out-of-town trip for the Balbalasan family makes the visit remarkable for the host husband, while the host wife noted enjoying an event that is usually attended by the local Filipino community and she shared it with her visiting mother. Such mutual feelings are shared by the visiting mother with other family members, including with her son-in-law's family as well as with her son regarding the family's migration to New Zealand:

My favourite was when I brought my mother to watch a concert of the Apo ${ }^{15}$. When I was still living in Singapore, I promised her to see "The Platters" live. I bought her a ticket in the Araneta Coliseum, but she was way too far from the stage. Here, she was up close and personal. She even got an autograph with the Apo and she really enjoyed it together with the other mothers who watched the concert. Of course, my family was also there. Immigrant-host daughter

Well, it's almost the same. It has become normal [hosting]. I think what I remember is that when we'd go out for a long drive, that is what I remember most. It is easier for me to recall those times when we'd go out of town for a long drive than those weekends that we'd just stay at home. It has become ordinary unless we go out, say, to Tauranga. However, if it's just Auckland or Kaipara which is just a one hour drive, then that's just a normal thing. Immigrant-host son-in-law

I think of my granddaughter's baptismal celebration. My daughter in-law was also there. My other son was there, too. Visiting mother

For the Isarog family, the silver wedding anniversary celebration bound the entire

\footnotetext{
${ }^{14}$ A Filipino composer and song-writer who was famous in the 1990 s.

${ }^{15}$ Popularly known as the Apo Hiking Society, a singing group considered the 'Beatles of the Philippines.'
} 
family together as it was witnessed by more than 400 guests. The immigrant-host family and their relatives have different tasks to make the event successful and the grandchildren emceed the event. The VRs also met some of their friends from their home province who had also settled in New Zealand. For the visiting sister, while she met her other relatives in New Zealand, there was a feeling of being "away" which is in contrast with the results of the study of Shani (2013) as relatives and not only friends may experience the feeling of being "away" while at the host's(s') home, even if the guest is with their immediate relative(s). Turning to the empirical studies that found that while a traveller may leave home, home does not necessarily leave the traveller (e.g., Duncan \& Lambert, 2003; Germann Molz, 2005; Larsen, 2008; Pons, 2003), the narrative of the guest could indicate that the sense of "home" may still be left behind if they consider their own family as "home." In this case, it is probable that the concept of "home" for the visiting relative is actually the significant others (the visiting sister's family) that were left behind in the Philippines, and as a result they cannot celebrate with her and their shared immigrant relatives in New Zealand on a special occasion. The visiting sister expressed regret due to her financial inability to bring them to share in the celebrations:

There's a lot that is memorable. Of course, that includes the wedding. I also remember bonding with my nephews and nieces. While I was there, I was also thinking it could have been better if my children experience what I saw there. Visiting sister

For the Matutum family who previously lived in the Middle East before settling in New Zealand, the feeling of being connected to their relatives was essential as they cannot travel to the Philippines very often as they have a big family. The winter season also gave them time together as a family as they cannot travel since their guests cannot easily withstand the colder weather in New Zealand. The visit also provides the grandchildren an opportunity to get to know their grandparents better as they left their former homeland at a very young age:

Probably when we travelled together, especially that grandfather told me that I was the first grandchild that drove for him. It kind of feels like a memorable moment for me. Immigrant-host grandson

There's no time that we don't go out - that's memorable. Also, when we go 
to church and I see how my grandchildren are used as an instrument by the Lord since they are members of the music ministry. I am very happy. Visiting mother

Going to nearby places with her guests makes the visit remarkable for the host wife in the Guiting-guiting family, while the visiting mother felt in solidarity within the family's spiritual practices and being reunited with her husband's faith. The sister of the host wife enjoyed her stay in Auckland and being toured around not only by Guiting-guiting family, but by her brother who has more time to take her out of town:

Although I did not join the trip with my relatives in Rotorua as my brother was the one who escorted them, I think it's the trips nearby [in Auckland] where we packed meals that we would take for a picnic -- then the picture taking. They were happy taking photos. Immigrant-host daughter

What is memorable for me was when my mother-in-law embraced our family's faith. Immigrant-host son-in-law

The most memorable is religion. I participate in our fellowship twice as we have bible study. Then, you meet many Filipinos. At my age, it's more spiritual. Visiting mother

Well, our reunion. Then, of course, visiting places which I can only see on Google - riding a cable car in Rotorua. Visiting sister

Overall, the findings from the immigrant-hosts and VRs demonstrate emotional solidarity, which is not only defined through shared beliefs and behaviour, but are also expressed through the shared identities in relation to their significant others. Whether it may be reinforced by communality, places, events, or situations, it is the co-presence of the significant other that produces a positive emotional experience, giving the immigrant-hosts and their VRs the sense of solidarity. The next section offers an indepth understanding of interpersonal interactions between immigrant-hosts and VRs through investigation of the cultural context of emotional intimacy and social exchanges. 


\subsection{Cultural identities in New Zealand}

To respond to the last supplementary research question: "How does culture shape the social interaction between the immigrant-hosts and VRs?", there are four thematic constituents related to culture that were identified: (a) filial piety; (b) the Filipino notion of care; (c) transplanting Filipina domestic roles; and (d) barriers to intergenerational solidarity. There seems to be a pattern within the immigrant-host family where parents may retain their traditional culture while immigrant-host children's behaviours are culturally modified as they may acquire the culture of a particular society in which they live. The implications of such cultural modification certainly influence the social interactions between immigrant-hosts in relation to their visiting relatives.

\subsubsection{Filial piety}

Filial piety involves the obligation of adult children to respect and care for seniors and the elderly with affection, responsibility, and gratitude (Lai, 2010). Regardless of religious and ideological traditions, as well as political and economic systems, filial piety dominates generational relationships in all East Asian countries (Villacorta, 2002) including the Philippines. This also concurs with the study of Jones (1995) that had results which indicate that social norms such as filial piety and respect for an elderly parent are strong among Filipino-American women and that also includes interpersonal reciprocity and obligation. While this study does not intend to silence the other members of the immigrant-host family, such as the fathers and children, filial piety is especially prevalent among immigrant-host mothers which becomes highly significant and noteworthy when they are interacting with their VRs:

I wouldn't be here [in this status] if they [my parents] didn't send me to a good school. They brought us up, right? When we are young, they were there for us. So, when they're old, I said to myself that it's now my turn as a daughter to look after them. [...] For me, I'm just paying back what my parents have given to me. [...] I told my mother that even when I am far away, my only shortcoming was that I was not there when she was sick. Immigrant-host daughter, Arayat family, individual interview 
You have been given the opportunity to go overseas. It's all a big achievement to be able to bring your family here. Immigrant-host daughter, Isarog family, individual interview

Immigrant-host mothers are grateful to their parents and for the sacrifices that they made in providing them an education which is benefitting them as they bring their social capital to New Zealand and enter into the workforce. In the context of university education in East Asia, the private sector dominates higher education by enrolling $80 \%$ of the student population including Japan, South Korea, Taiwan, and the Philippines. In particular, private institutions in the Philippines are greatly profiting as they depend largely on tuition fees (Altbach \& Levy, 2005) while Filipino parents struggle to try to make both ends meet for their family. As these Filipino immigrant women embark in another phase in their life in New Zealand, which cuts across long-time and place, recent migrants in the study demonstrate through their benevolence that the notion of reciprocity should definitely not be confused with repayment, as the act of hosting their relatives is giving of oneself:

I will give everything even if I cannot surpass what she has given me so that she can be happy for me as her daughter. Immigrant-host daughter, Makiling family, individual interview

Filipino children are expected to possess a sense of utang na loob (debt of goodwill) towards their parents for having reared them, as reflected in their respectfulness and honouring family obligations. Otherwise, they will be known as without hiya (shame) or without utang na loob (who does not act with decency) (Alampay \& Jocson, 2011). On the contrary, whether or not the immigrant-hosts and VRs refer to the responsibility of hosting overseas as utang na loob, it may be immaterial because parents and children would recognise that bond (E-mail communication with Dr. Leonardo de Castro, National University of Singapore). Strong emotional ties to their former homeland's culture and its values are manifested as daughters bring their parents to New Zealand:

My hosting behaviour still reflects being a Filipino. As a Filipino, you show gratitude to your parents or maybe out of love because you wouldn't be who you are right now if not because of them. You tell yourself that they are the ones who took good care of you and send you to school. It's my way of 
showing my appreciation and humility to them. The only aspect of being a New Zealander is my status as a permanent resident. Immigrant-host mother, Matutum family, individual interview

Acts of kindness or generosity are not something that VRs/Filipino parents would expect from their children. Parents regularly expressed a preference for staying at home to serve the needs of the household and would oppose extravagant spending in order for the family to conserve the money to support the household. Even though they may be opposing their children's(s') fiscal decisions, this is actually an expression of solidarity on the part of the parent(s)/guest and they are being a caring relative, as they do not wish to abuse the fiscal resources of their immigrant-host family:

My children treated me for dinner at Skycity and it cost $\$ 65$. They have been bringing me to fancy restaurants. They treated me like a visitor and I have resisted because it's a waste of money even if they pointed out that they wanted me to experience the good life they have here. I guess it's their way of showing utang na loob [debt of goodwill]. I always argue and told them, "I'll just stay here at home and do babysitting." Visiting mother for Balbalasan family

Similarly, even an immigrant-host father shared a similar sentiment in relation to filial piety which is expressed in relation to his parents-in-law as his own parents are no longer living:

I think it's a once in a lifetime for you to host them and for them to experience life here. Especially for me, I do not have my own parents anymore. Sometimes, you think that it would have been nice to bring them here. I think we owe everything to our parents to show them how successful you are here and share that with them. Immigrant-host son-in-law, Arayat family individual interview

In essence, hosting is influenced by the ties that still bind immigrant-hosts with their relatives even though they now reside far away in New Zealand. Immigrant-host parents still uphold filial piety which underlies intergenerational relationships regardless of where and when the parent-child interaction takes place (Ho, 1998). They still uphold typical Filipino values, not so much as a way of "paying forward", but because they are still in full solidarity with their relatives. Such emotional fidelity is accurately expressed in a popular Filipino saying: "Those who do not look back to where they came from will not reach their destination." 


\subsubsection{Notions of caring for Filipinos}

Caring is a cultural construct as for "what is caring" for one culture may not be so, to another. In relation to filial piety as expressed by immigrant-host parents presented earlier, this cultural value is better understood as a commitment among family members, which works both ways, and is therefore being reciprocated by their VRs. This is evident among the social interactions between immigrant-hosts and VRs whose main purpose for making the visit is to provide childcare. What is common is that these immigrant-host households are young with families that have children who need to be supervised and that both parents feel the need to work for the family due to socioeconomic pressures. On the other hand, as a solution to the personal and financial conflicts facing the host families with young children, VRs made themselves available to provide childcare.

VRs from the Philippines have a great sense of familial responsibility and they are willing to endure major sacrifices for the opportunity to advance their family's situation. They are often willing to leave the Philippines to support young immigrant relatives with children, sometimes despite their physical limitations (age or infirmity), which can overwhelm one's emotions when one is faced with accounts of the VRs' commitment to their kin. A VR's sense of duty to their immigrant-host family is frequently beyond measure. In addition, the traditional role of childcare becomes a responsibility of women and the myriad of responsibilities of motherhood can spiral to a point where it can affect the mental health of the mother particularly for first-time mothers and where immediate relatives are unavailable to assist her as she is residing in a new homeland. VRs, through a long visit, are able to provide support to an immigrant family during this crucial and needy phase for a young family and also pass on their traditional practices in childcare:

Sometimes, she does agree with me as to how one should take care of a child. I apply aceite de manzanilla ${ }^{16}$ at the back of the toddler to stop coughing. [...] Also, it's effective when you have stomach pain. One thing I didn't like is that she baths the child with cold water. Now, she doesn't do it anymore. I told her to use lukewarm water. Visiting auntie for Apo family

\footnotetext{
${ }^{16}$ Chamomile oil
} 
However, even when families can afford the costs for childcare as a public service, VRs share their cultural views about babysitting and child rearing. As discussed earlier in section 5.2.1, childcare as a commitment requires trust between the parents and the caregiver:

Because in a day care, they just leave the children playing and they sometimes get injured or need a cast [due to a break]. You know we Filipinos, we really take good care of our children, right? Visiting mother for Balbalasan family

In the same vein, VRs often make themselves available to care for their family during critical phases, while noticing some differences that caused concern in hospital-based post-natal care in the host family's new homeland (of which an immigrant-host husband may also not appreciate or understand the significance if he does not have a medical background):

Of course it is important because I saw my daughter's situation especially when she gave birth. I especially didn't like that they give you a bath after giving birth. It was very cold. If I wasn't there, my daughter would have been really chilled so I gave her porridge. Visiting mother for Balbalasan family

While the structure of Filipino families becomes nuclear once immigrants move to their new homeland, these immigrant-host families ideally could still use a relative as a substitute for childcare as they probably have more trust in an immediate relative, especially in their parents when it comes to childcare due to their trust gathered from their own childhood experiences. Interestingly, there is also a gendered dimension in childcare as an immigrant-host mother explained the need to bring her own parent above anyone else:

I know that my children will be in good hands with my mother [...] Of course, as a woman in need of help, you want to seek assistance from your own mother. You cannot say you need help from your mother-in-law. It is still different because you are more comfortable with your mother especially that I was a caesarean. She takes good care of you and will feed you well. Immigrant-host daughter, Balbalasan family

In response to the filial piety accorded by an immigrant-host (in section 5.5.1), it is reciprocated also by the sharing of oneself on the part of a VR and also in making the long trip to New Zealand. Family and kinship reciprocity is an ongoing process and is therefore an intergenerational relationship (from grandparents to grandchildren). 
Earlier, the notions of caring for Filipino families were discussed in relation to childcare and maternal care that VRs provide for immigrant-host families. The conceptualisation of care is a cultural construct which is also gendered as both immigrant-host mothers and their relatives may still share common beliefs and behaviours which both of them brought to New Zealand. The next sub-section will focus on immigrant-host wives in relation to domestic hospitality.

\subsubsection{Transplanting Filipina domestic roles}

Within the domestic sphere, Filipino women are maintaining both their obligations with their families in New Zealand and also with their relatives in the Philippines. This sense of international familial responsibility may be dictated by the culture of these women or, as one may assume, by the important role of familial dynamics which can depend on their birth order within the family. The respective authority of offspring within the Filipino family descends on the basis of their respective birth order or ages. Traditionally, a ladder type of authority exists where the older children are dominant over the young ones. The eldest child, in particular, has a quasi-paternal status and has authority over all their junior siblings. He or she is looked-up to with respect by his or her junior sibling(s) as an authority figure and has a role of a second tier parent within the family (Medina, 2001, p. 29). The immigrant-host mother for the Isarog family expressed her obligations to her family in the Philippines prior to her moving to New Zealand:

Immigrant-host mother: We're seven and I'm the eldest. My sister is the second one and we have five brothers. When we were growing up we were close. But now that we're apart, I feel that is why this visit also binds us because, when I left, she took on looking after my parents because I used to do that role. And now she is the next in line. [...] My fear before when I first came here because she was not really very responsible then. She was just relaxed and she knows I am a strong woman. Before, she just depended on me. Now that I am here, I felt like she has matured.

While the majority of the VRs were parents of immigrant-host mothers, there are even more expectations for women in hosting for their husband's relatives as expressed by the immigrant-host mother for the Arayat family: 
Immigrant-host mother: It's different when it's my family who will visit me here. But if it were my husband's family, it's gonna be "different." I guess that's how things work in the Philippines.

Interviewer: You mean you really prepare very well?

Immigrant-host mother: Yes, if it was my own family visiting, l'll be more relaxed. But if it were friends or relatives from my husband's side, you're more -- should I use stressful?

Interviewer: You mean you're pressured.

Immigrant-host mother: Yes. Of course, it's different hosting for your own relatives since you were raised and grew up with them. It's different to host for relatives from my husband's side since you don't have blood relations. You cannot simply give them a task at home.

In another instance, a similar sentiment is shared by another host wife when she hosted the auntie of her husband:

As a woman, you have to make your guest comfortable at home considering that she is a relative of my husband - because usually, it's the female who should be welcoming the guest. Immigrant-host wife, Penablanca family, group interview

However, not one immigrant-host husband expressed any difficulties in hosting for their VRs. Whether there is a blood relationship between a female host and the guest, or many tasks related to household duties and domestic hospitality and may place an additional burden on women when hosting for a guest, regardless of whether it is a relative or a friend (as earlier shown on Chapter 4).

\subsubsection{Barriers to familial solidarity}

The first generation immigrant-host family members, notably the parents, have been raised in the Philippines with those cultural norms, traditions, and values that bind them communally with their VRs. However, children of immigrant-host parents do not necessarily identify themselves with their relatives' culture as they are also shaped by and share in New Zealand culture. Therefore, there can be conflicts from the expectations of elders and stressors that surface between the collision of two different value systems, specifically between the Filipino and New Zealand cultures. These 
conflicts can create internal discomfort and frustrations that can create an opposite reaction to hospitality, that being resistance and hostility to the ways, expectations, and traditions of the former homeland.

While ties are maintained between the first generation Filipino immigrants and their relatives in the Philippines through VFR travel, transnational migration scholars predicted that the salience of ties could rapidly decline among their children (Levitt \& Jaworsky, 2007). This is revealed among the interviews conducted with grandchildren as they interact with their guests (usually grandparents). In the first example, while the grandson in the Pulag family spent his primary and secondary schooling in the Philippines before moving to New Zealand, he is also discovering his identity as a New Zealander in expressing his own individuality which apparently may have offended his grandparents, who have a different view:

Immigrant-host grandson: For me, I enjoyed my grandparents visit. I just had a petty argument with them during their previous trip because I coloured my hair and they didn't like it.

Interviewer: What colour?

Immigrant-host grandson: Ice blonde.

Interviewer: It's like yellow? Or blonde?

Immigrant-host grandson: Blonde. I really went to that phase [in life] before.

Interviewer: [Sort of a] Fashion statement?

Immigrant-host grandson: It's like [thinking] -- not really a form of rebellion to your parents or anything. In fact, they don't mind because New Zealand is an open society.

Interviewer: Are you referring to how you define your own individuality?

Immigrant-host grandson: Yeah. I went through that phase as part of being teenager. I wanted to explore and try new things. I enjoyed it [colouring the hair] but they did not like it and for them, it's a waste of money. But I think, since we are in New Zealand, we can do or express ourselves which we can't do in the Philippines. It's a way of not conforming while here in New Zealand.

Interviewer: So, you have just ignored them? 
Immigrant-host grandson: In a way. They disagreed and even made a comment on Facebook. From my point of view, I am old enough to decide what I wanted to do with myself -- since I was already working at that time. While we have certain misunderstandings, I have become more mature and they see how I have progressed in my career of which they are proud.

In the second example, there is a feeling of animosity for the grandson in the Matutum family as they used to live in the Middle East where his parents previously became migrant labourers. Even though he was educated in a Filipino school overseas, he never had the chance to live near his grandparents making it difficult to live with them under one roof during a visit:

Interviewer: How would you describe the experience when your grandparents were accommodated here?

Immigrant-host grandson: Well, it was a bit awkward for me.

Interviewer: In what sense?

Immigrant-host grandson: I think it's because - it's like we never had the chance to spend time together before.

In the third example, children of Filipino immigrants who grew up in New Zealand even find the differences between their own evolving sense of culture when placed against the former culture of their parents, which may have implications for hosting relatives from the Philippines:

One thing peculiar that I find within the Filipino culture is that they are willing to share or give up their room for their guest. Immigrant-host grandson B, Isarog family, group interview

To be honest, I am not very good at doing pagmamano ${ }^{17}$; it's because I was brought up here. Ever since I was like, kid, we don't really do it but, since my grandparents were here visiting, I even had to tell my [New Zealander] boyfriend to do the same when he visits me. Immigrant-host daughter, Arayat family, individual interview

Using the premise of Protacio-Marcelino (1996) in relation to the interpretation of Filipino children in the context of diaspora, these immigrant-host children are Filipino-

\footnotetext{
${ }^{17}$ The Filipino tradition of pagmamano involves kissing an elder's hand or raising the hand to touch your forehead.
} 
New Zealanders and are no longer Filipino, which some VRs may not be able to understand. Hence, cultural-based conflicts may arise between grandparents and grandchildren. The latter are both Filipino and New Zealander who have different cultural experiences growing up in New Zealand. However, they can still lay claim to the Filipino cultural identity (different from national identity) because both their parents are Filipino and they may still speak or at least understand the Filipino language.

The immigrant-host mother is uniquely positioned in transmitting the cultural values of Filipinos in New Zealand and strives to avoid conflicts between grandparents and grandchildren, especially as maternal relatives have longer visits with the host families:

When you're with your parents even in your own house, you still follow the -- where they want to go -- whatever the house rules -- whatever your children like. They have the control during the time they were here in New Zealand. It's like, we step back a bit -- for them to enjoy -- so that you wouldn't clash with them -- compared in the Philippines, at home -- so it's like you try to adapt with them. You bend your rule -- to make them more comfortable [...] just like in the Philippines -- the way you eat together [...] you wake up early and retire earlier. Immigrant-host mother, Pulag family, individual interview

This section has provided a multidimensional picture involving culture that defines the identity of immigrant-hosts in relation to their social interactions with their VRs. In particular, the cultural context of the social interactions are intertwined and influenced by the social exchanges and intimacy between the immigrant-hosts and VRs. Immigrant-hosts parents still retain the value of filial piety as an obligation to their VRs. Such cultural value is reciprocated by the sense of familial responsibility or duty on the part of the VRs. A gendered dimension of social interactions also shows that immigrant-host wives/mothers maintain their traditional role within their family in New Zealand while maintaining their familial obligations in the Philippines. On the part of immigrant-host children, the acculturation process unfolds at different times depending on their residency period which may become a barrier towards socialising with their significant others. The next section will now answer the main research question related to the interpretation of the meanings of the visit for relatives. 


\subsection{Meanings of the visit among relatives}

The meanings of social interactions are textured by the situations and objects of those experiences and are composed of the meanings they have; in this case, the overarching experience being "the visit." The meanings of gestures made by the immigrant-hosts are the responses to the courtesies of the visiting relatives and vice versa. Individuals behave according to their interpretations of the meanings derived from and the experiences gathered in their day to day world. In this section, the immigrant-hosts and VRs provide subjective meanings on what they believe and not just on what is objectively true about their social interactions but in how they subjectively interpret the goings on and events based on their past. Therefore, their interactions are socially constructed through their interpretations and constructs from their past.

In order to address the main research question for this chapter: "How are social interactions between immigrant-hosts and their visiting relatives understood and interpreted by them?", the different perspectives of the family members as immigranthosts and those of the visiting relatives are used to illustrate the meanings of the visit. First, as immigrant-host mothers interact with their parents, the meanings of the visit are encapsulated in filial piety, respecting intergenerational ties, and expressions of gratefulness for childcare support. Second, immigrant-host fathers communicate their sentiments in relation to their VRs (parents-in-law) which reflects their sense of pride and reciprocity. The host mothers' expressions can be summarized as communicative, active and demonstrable whereas the host fathers' expressions could be described as implied, subtle and egoist. Third, immigrant-host children provide several meanings depending on the quantity and quality of past interactions which affects how well they know their visiting family. Finally, it is altruism or some caring behaviour(s) that guide the social interactions of visiting grandparents with their relatives in New Zealand. Other members of the party of VRs related similar sentiments.

Underpinning the cultural value of filial piety (explained earlier in section 5.5.1) are the immigrant-host daughters who celebrate the life they have, not only with their husband and children in New Zealand, but also continue sharing it with their relatives in the Philippines. This is particularly expressed by the immigrant-host daughter for 
Sierra Madre who has hosted her father many times (and her mother who is now deceased). Similarly, the immigrant-host grandson, who grew up with his grandparents and values his grandfather very much and more so now that they are no longer regularly together:

I saw how happy he was with my accomplishment. Although he is far away from us, he knows we are in good hands; that our family here did not live in destitution. He always thinks about his grandchildren. Immigrant-host daughter, Sierra Madre family, individual interview

His presence was very important since I am new to New Zealand and he was with me so, it feels weird that he is no longer here. Immigrant-host grandson, Sierra Madre family, individual interview

While the visiting grandfather may have confused his previous trips to New Zealand with the latest visit, or may no longer remember all the places that he has visited with his children or grandchildren (due to old age), it is the company of his family members that endures and makes him feel fulfilled from traveling and visiting:

It's really important to see my grandchildren and children - you feel complete and happy. Visiting father/grandfather

Similar to the immigrant-host wife/daughter for the Sierra Madre family, the immigrant-host mother/daughter for the Matutum family shares a similar sentiment. Filial piety is also manifested in how eager these immigrant-host daughters are to share being in her "new world", New Zealand, with her parents and in letting them know how well she has progressed or succeeded overseas which is a direct result of their efforts and having been raised by them. These sentiments are also shared by the host-husband in the bringing of his in-laws to New Zealand and in the re-establishment of the ties not only with them, but also between his in-laws and his children:

The visit is important because they are my parents. For me, my life is not enough to offer them. I have achieved and experienced everything here [the good life] and I want to share that with them. Immigrant-host daughter/mother, individual interview

It's a dream come true. We want to show how beautiful this country is and to introduce our children to them. Immigrant-host son-in-law, individual interview 
As for the immigrant-host grandchildren in the Matutum family who were separated from their VRs at an early age, re-acquaintance is expressed:

We had the opportunity to meet and get to know them better. Immigranthost granddaughter, individual interview

We got to know them and it's been a long time since we haven't seen them. Immigrant-host grandson, individual interview

Beyond the significance of traveling to New Zealand for the first time, the guests of the Matutum family are satisfied, not only to see the place, but because they see how their relatives are enjoying a better quality of life overseas. Such concern also provides VRs with peace of mind knowing that their host family is in a better situation in their new homeland:

We are glad to see our daughter's situation and her family. They are in a better position in New Zealand having good jobs. For a parent, that's important. A parent would always want their children to be successful in life. Visiting father, individual interview

It's important to visit them because we are concerned and have been thinking about their situation including that of our grandchildren. I was so happy because they are spiritually active. Visiting mother, individual interview

While immigrant-host daughters do not have an outright obligation to invite and pay for their parent's $\left(s^{\prime}\right)$ travel (or for other members of the family) to visit them overseas, they frequently do. These immigrant mothers through their invitations and investments in hosting become the bearers of intergenerational solidarity as they bridge the relationship between their family in New Zealand and their relatives in the Philippines. The instance of motherhood often incites recognition of the importance of the matrilineal bonds within the family and they try to foster or nourish the sense of uniqueness in the relationship that grandparents have with their grandchildren, even if they do not live in the same country. Despite her busy schedule at work, the immigrant-host daughter/mother and the rest of the members of Isarog family prepared a big wedding celebration, not only for their friends in New Zealand, but also for their relatives from the Philippines: 
It's important not only because we can afford it. It's even more important that they were able to witness my wedding. Immigrant-host daughter, individual interview

They were able to see our family's situation. They have seen what we achieved here in New Zealand. That's also something that we would like them to see. Immigrant-host son-in-law, individual interview

The occasion was important as we saw our auntie and see how grown up we are compared to eight years ago when we are just kids. Our relatives have seen how we have grown up and taking responsibilities. Immigranthost son A, group interview

There's just a big difference in virtual communication versus meeting them in person. You know, even if it was a short amount of time -- even if it was just a week or three days, that physical connection means more than anything. Immigrant-host grandson $B$, individual interview

The guests of the Isarog family, particularly the grandparents, are delighted to celebrate with their relatives in New Zealand. Despite the logistical challenges and physical limitations of the older visitors that makes long travel a challenge, seeing their immigrant relatives again after many years becomes a source of endless happiness as the reunification rejuvenated their relationships with their immigrant-host family as they now belong to a new world and culture:

I was very excited to meet my grandchildren. As grandparents, we were excited to be with them. That's very important. Also, because I wanted to see if they have good living conditions there. Visiting mother, individual interview

Very important, because it has been years since we had the chance to talk [personally]. Your longing for them was healed after you met and bonded with them, even for a short period of time. You are concerned that travelling [to New Zealand] is expensive, but once you are there, it's gone after seeing your children who are far from you. Visiting father, individual interview

Even the visiting sister of immigrant-host mother for the Isarog family felt in solidarity, not only in seeing the success of her relatives, but also in being in union with them in New Zealand:

It's very important to be with them. I wanted to see their living conditions. I saw how fortunate they are with their [economic] status. We were happy there because we were all together [in New Zealand] which cannot be measured by money. Visiting sister, individual interview 
For the Guiting-guiting family, the immigrant-host wife has a sense of pride in hosting her relatives and by being able to show how the quality of their family's life has improved overseas. However, it is the sense of reciprocity that guides the social interactions of the immigrant-host husband (son-in-law). The immigrant-host husband is concerned for the needs of the VRs as they provide care for his children. In this particular host family, it is the grandparents who are also making sacrifices by providing childcare:

Our family is able to share what kind of living we have here which they appreciate. We become an inspiration especially for my siblings, like my sister who visited. She saw that we are not rich in New Zealand yet we have a decent life. Immigrant-host wife/daughter, individual interview

The visit is very important not because of the personal gains that I can get from it. It's returning the favour by showing kindness to them. Immigranthost son-in-law, individual interview

I saw my children there and their respective families. We had the chance to be together - but I don't want it [to be overseas and away longer] all the time because I am the only one left [no family around to mind her] in their home. Visiting mother, individual interview

The visiting sister of the immigrant-host wife of the Guiting-guiting family is the eldest among the maternal siblings. She was very happy to see that her siblings are in a better economic position in New Zealand. Above all, her main goals are the strengthening of the ties with her immigrant siblings and getting to know her immigrant nieces or nephews:

Seeing my relatives, fill the gap - it strengthens our relationship more as siblings. Then of course, I think it's a two-way as it strengthens our bond. Then, I also wanted to see how they treat their children and conduct their family life. I also had to see my brother because he is the youngest. Visiting sister, individual interview

Likewise, the immigrant-host Pulag family members value their relationships with their significant others back in their former homeland and are therefore willing to continue their intergenerational ties with their relatives in the Philippines:

Hosting them was very important for me because they are getting older. I want them to enjoy their time with my children - because they are afar - 
and my older brother is also married but has no kids - so, my children are their only grandchildren. It was difficult for them that we migrated to New Zealand. Immigrant-host mother/daughter, Pulag family, individual interview

It's important to touch-base with my mother-in-law and that you're together in person, you share stories together about what is happening in the Philippines. Immigrant-host father, group interview

It's such a big deal hosting because first it has been many years that we have seen our grandparents - my siblings were still very young when we migrated. We miss them - and it's a different experience that you are with them as compared to when you chat on-line. Immigrant-host grandson

It's not about the money or the long hours of travelling [to New Zealand]. It does not detract from the experience you have of personally of being with them. Visiting mother

Immigrant-host couples are also very grateful that their relatives sacrificed their time to assist them with childcare but the couple may not share the same expectations and meanings of the visit. For example, the immigrant-host wife calls for the immigranthost father to reciprocate in kind for their guests' sacrifices in providing childcare:

Since my mother is getting older, I wanted to spend time longer with her. Of course, I want her to see our lives here. Immigrant-host daughter, group interview

I wouldn't say that I tried to be nice to them, but I was really very nice because it's not really their responsibility or obligation to come here to help us. They did that as a favour which I need to return. So, if there is anything they need in the Philippines, we will find a way - that was what I promised them. Immigrant-host son-in-law, group interview

I saw my daughter and my grandson. Visiting mother, individual interview

I am really happy seeing her [niece]. I really care for her that I even packed a meal before she goes to work. I tell her I will cook whenever she is hungry. That's who I am. Visiting auntie, individual interview

In addition, inter-generational ties are dearly missed by the immigrant-host families as many do not have other family members in New Zealand to help them within their household. Visiting relatives allow them to benefit from the visit in terms of receiving 
help with child care. The Makiling family expressed great appreciation for their visiting grandmother and they attempted to properly provide for her all of her needs while she took such good care of the children during her visit:

Hosting is important because you get to spend time together while nurturing and deepening the relationship with my mother. She's also part of my children's life and that they get to know their grandmother since they don't get to see her that often. Immigrant-host daughter, individual interview

At least my mother-in-law got the chance to travel and look after her grandchildren while she is still physically strong to travel. Immigrant-host husband, individual interview

It's very important because I have become happier. I made them happy. We knew that they were longing for their father [tears started to flow]. The pain that they felt somehow subsided since I visited them [...] Hosting me was very important because I felt how much they love me. Even if they were working, they spent money to bring me there. I can't find anything negative, especially of [name of son-in-law], he is very kind. I guess they are just returning the favour of how I have treated them. Visiting mother for Makiling family

Similar to the Makiling family, the host-couple for the Balbalasan family also express a similar interpretation of their social interactions with their VR in respect of continuing their ties with relatives in the Philippines:

First of all, it's very important, other than we are benefitting [from their care of our children]. Second, this is a way for us to treat her [my mother], by taking her elsewhere in New Zealand. It's just like the rich in the Philippines who can afford to travel to Hong Kong with their family; I will tour my parents. Here, you have a purpose [family visit] while at the same time the opportunity for her to see New Zealand. Immigrant-host daughter

The visit is important because when we got married, we immediately left the Philippines and it is only here in New Zealand that I got to know my inlaws. Immigrant-host husband, group interview

Comparatively, the social interactions between immigrant-hosts and VRs reflect that it is not only the host who looks after the wellbeing of the guest. Rather, the act of caring maybe also expressed by a so-called "guest" as indicated by the visiting mother/grandmother for the Balbalasan family. While immigrant-host families share their new world with their VRs, it is in fact a reciprocal exchange as the VRs may sacrifice by staying in New Zealand for a longer duration. This is in contrast to the 
normative host-guest paradigm as it is not only the hosts who have concern for the wellbeing of the guests but the VRs also return in kind:

Of course it is important because I saw my daughter's situation, especially when she gave birth. Visiting mother

A similar insight from another visiting mother/grandmother for the Banahaw family was seen as she voluntarily paid for her trip which is an expression of her care and concern for the wellbeing of her relatives in New Zealand:

I visited them because it's been a long time since he [my son] left the Philippines. So, I had to visit them and see how they are [doing] in the first place. You know [name of daughter-in-law], she also got sick. I want to see how they are, their financial status, and health, how they cope -- their wellbeing -- that's the first thing I want to know. Visiting mother

On the other hand, the visit gave the immigrant-host son and his family the opportunity to share the life they have in New Zealand. Meanwhile in the Banahaw family, the immigrant-host wife/daughter-in-law may have been adjusting to their lives in their new homeland after recovering from health issues. Such social interactions also paved the way for them to honour her mother-in-law and to incite the immigrant-hosts and their children to make a return visit to the Philippines in the future:

I wanted her [my mother] to see our lives here first hand so that she would understand how busy we are. Immigrant-host son/father, individual interview

My mother-in-law's visit is very important because when she celebrated her $70^{\text {th }}$ birthday, we were not able to go home. Now, she has reminded us to return to the Philippines on 2015 for her $75^{\text {th }}$ birthday -- so we have to save money for that. Immigrant-host wife/daughter-in-law, individual interview

As for the Penablanca family, there are no hesitations to host their auntie or other relatives who may visit them in the future. Such social interactions among the host family and their distant relatives allows time to become compressed since they did not have much chance to bond while in the Philippines; both the nephew and his auntie have the opportunity to interact during a visit in New Zealand: 
My auntie's stay was a delight. We didn't have problems or any dull moments, which is good. Immigrant-host nephew, group interview

We want both sides of our family to experience traveling to New Zealand and stay in our place. I had no interaction with Auntie prior to her coming here. After the visit, we became close. Unlike on my family's side, my husband was telling me that their clan is not really that close and they only get to see one another during important occasions. Immigrant-host wife, group interview

First of all, the visit binds my relationship with the couple. I got to know them better. While I know my nephew, I came to know his wife deeper when I stayed with them. Visiting auntie

Undoubtedly, the immigrant-hosts and VRs social interactions as phenomena are not objective entities. Rather, they represent the product of relationships made from subjective meanings. The different illustrations of the meanings of the visits of immigrant-hosts and VRs form the basis of their inter-subjective understandings of a particular situation - in this case, the visit. The interpretation of the social interactions of the immigrant-host mothers are expressed through filial piety and continuing the intergenerational ties that connects their relatives in the Philippines with their family in New Zealand. In contrast, the social interactions of the immigrant-host fathers/husbands are articulated by mutual exchange or reciprocity with the VRs/inlaws and are expressed by showing that they have the adequate material resources to properly host and what they have accomplished in their new homeland. Immigranthost children provide varying interpretations of their social interactions depending on their affinity with their VRs, but by and large encompassed re-acquaintance and reciprocation to the relative's(s') visit through their co-presence and time spent for them. In comparison, the interpretation of social interactions for visiting relatives as "guests" are oriented towards the wellbeing of their host(s) which is an expression of gratitude, altruism and compassion.

\subsection{Conclusion}

Social interactions generally influence the depth of the shared experiences of the immigrant-hosts and VRs by re-establishing and renewing family bonds through family 
reunification and fulfilling obligations within the family. They are multi-faceted, dynamic, and are entangled as immigrant-hosts and their VRs are interdependent and they mutually benefit by the other's co-presence. The social obligations to travel on the part of the VRs and for hosting by immigrant relatives create dynamic and reciprocal relationships through the fulfilling of duties among the family. Such social exchanges are focused on nourishing and maintaining relationships between family members.

The solidarity made as a result of the co-presence of family members produces an affirmative emotional experience for both the immigrant-hosts and their VRs. As such, emotional solidarity is not only expressed through shared beliefs and behaviours, but also through the shared identities of their kinship. However, culture affects and influences the nature of social exchanges and the emotional solidarity between immigrant-hosts and VRs. Understanding the experiences requires situating the cultural context of the relationships of immigrant-host families and VRs and how it shapes and enmeshes the social interactions between them.

The logistical aspects of visits in separate countries, which is the reality of immigranthost's(s') and VR's(s') social interactions may cause the participants to reciprocate in various ways that occur at discontinuous moments in time. Thus, certain mutual exchanges that may or may not be contemporaneous with each other such that the immigrant-hosts and VRs may not anticipate or expect an immediate reciprocation as they express concern over the welfare and needs of the other. A multiple-perspective approach is utilised that brings together the voices of the different actors engaged within the social interactions between immigrant-hosts and VRs and highlights the subjective meanings of social interactions for the immigrant-hosts and VRs that are socially constructed. The next chapter (Chapter 6) will deal with combining and contrasting the results of Chapters Four (Analysis of the social interactions between immigrant-hosts and VFs) and Five (Analysis of the social interactions between immigrant-hosts and VRs) through qualitative metasynthesis as well as theoretically interpreting the immigrant-host's( $\left.\mathrm{s}^{\prime}\right)$ and VFR's(s') social interactions. 


\section{CHAPTER 6 - Qualitative metasynthesis and theoretical interpretation of the social interactions between immigrant- hosts and VFRs}

\subsection{Introduction}

This chapter discusses the theoretical interpretations of the findings that resulted from the analysis of the social interactions between immigrant-hosts and their VFRs. This study is the first of its kind to examine social interactions in the context of VFR travel and from multiple perspectives, in particular the interactions from the perspective of the immigrant-host families and their VFs or VRs. Of equal note, in the past little attention has been paid to the interpretation of social interactions between and among the multiple actors involved in VFR travel. Prior to addressing the broader questions in this thesis, this chapter will also expand upon the conceptual framework in an illustrative manner based upon the results of two earlier chapters. This will provide structure that will aid in synthesising and in explaining the theory and results of the research.

The organisation of this chapter is structured in accordance with the research questions presented in section 1.5. However, prior to addressing these questions, the conceptual framework that was developed and presented in Chapter 2 is first revisited in section 6.2. in order to reconceptualise or reframe the phenomenon under study, specifically the social interactions between hosts and guests in the context of VFR travel. This strategy will empirically examine the conceptual framework with the purpose of understanding the social interactions of the immigrant-host families and their VFs as well as the social interactions of the immigrant-host families and their VRs. Then, the succeeding sections from 6.3 to 6.7 will address each research question of the thesis through a qualitative metasynthesis approach to review and interpret the results while integrating the findings from Chapters Four and Five with the broader literature and highlighting similarities and differences. Qualitative metasynthesis is an interpretive integration of qualitative findings - either conceptual/thematic descriptions or interpretive explanations (Sandelowski \& Barroso, 2007). This chapter addresses the main research question of the thesis: "How are social interactions between immigrant-hosts and their visiting friends and relatives understood and 
interpreted by them?" Underpinning this question are the following supplementary questions:

1. What is the nature of the social interactions between immigrant-hosts and their VFRs?

2. What are the social exchanges between the immigrant-hosts and their VFRs?

3. How do social interactions reflect emotional solidarity between the immigranthosts and VFRs as expressed by their shared beliefs and behaviours?

4. How does culture shape social interactions between the immigrant-hosts and VFRs?

The first supplementary research question compares and contrasts the nature of the social interactions between the two principal sets of actors examined in this study, the immigrant-hosts and VFs and the immigrant-hosts and VRs. However, what is similar is that both social interactions are complex and dynamic involving the various members of the hosting family together with their respective guests (VFs or VRs) and in varying degrees according to the dynamics of their relationships. Addressing supplementary research questions 2 and 3 utilises social exchange theory and the theory of emotional solidarity to provide an improved understanding of the social interactions between immigrant-hosts and VFRs. This innovative approach permitted interpretation of a multifaceted phenomenon as no single sociological perspective can reasonably claim a monopoly in providing an understanding within tourism research (Dann \& Cohen, 1991 ) including the social interactions between first-generation Filipino immigrants in New Zealand and their VFs or VRs from the Philippines. As tourism is identified as an agent of cultural invention (Jamal \& Hollinshead, 2001), culture becomes an essential element in social interactions, which brings into focus the relationship between the hosts and the guests. This is therefore addressed in the fourth supplementary research question where culture shapes the social interactions between these actors. Consequently, the main research question is addressed using a hermeneutic phenomenological perspective which facilitates an understanding of the meanings objects hold for the perceiver(s), but also in seeking to understand the relationships between them including tradition, culture, and social settings (Pernecky \& Jamal, 
2010). The next section will expand on the conceptual framework based on the empirical findings of the study contained within Chapters Four and Five.

\subsection{Reframing the conceptual framework on the social interactions between immigrant-hosts and VFRs}

The absence of an established framework on social interactions between hosts and guests in the context of VFR travel led to examining the phenomenon being researched using different sociological theories. The main strength of this thesis lies in carrying out the research to help fill this gap. Furthermore, the prior lack of conceptual understanding of host-guest interactions in the context of VFR travel as a phenomenon has systematically situated this thesis within tourism studies and it also draws upon scholarly literature that addresses family and friendship.

Figure 6.1 expands upon the conceptual framework in section 2.6 and is based on the empirical evidence provided in Chapters Four and Five. After analysing the social interactions between the immigrant-hosts and VFs (Chapter Four) and the immigranthosts and VRs (Chapter Five) separately, the findings identified new concepts that allow better interpretation of the cultural context and the meanings and understandings of the interactions between hosts and guests. In addition, this thesis has utilised the conceptual lenses of social exchange theory and the theory of emotional solidarity. Theoretical explanation involves efforts to visualise a particular phenomenon as an example of a more fundamental process that is depicted in abstract models (Turner, 1988).

Since there are no established concepts for interpreting the social interactions between hosts and guests in the context of VFR travel as a phenomenon, the conceptual framework that was developed in this thesis was reframed with the empirical results of the study. Drawing on the data that emerged in Chapters Four and Five, the conceptualisation of the immigrant-host's(s') and VFR's(s') social interactions were reframed using the two theories: social exchange theory and the theory of emotional solidarity. Given the broad themes that had emerged from the social 
interactions between immigrant-hosts and VFRs, Pearce (2012) elaborated on the functions of operationalising the conceptual framework:

The balance in emphasis between developing a conceptual framework and applying it in empirical work will often reflect the complexity of the constructs and concepts being brought together, the extent to which these have been clearly articulated and the degree of consensus about their use. Where the concepts and constructs are complex, as yet poorly developed and/or no general agreement on their use exists, the emphasis will generally be on developing the framework by identifying the concepts and showing the relationships between them (p. 38).

Reframing the conceptual framework was necessary for the following reasons. First, while both social exchange theory and the theory of emotional solidarity are used to interpret the relationships between hosts and guests, there is still an element of "othering" where the interaction is a meeting of strangers. However, social interactions between friends and family members are different as they are familiar with their respective relationships. Second, these theories were not yet examined in relation to the social interactions of hosts and guests that underpin VFR travel. Third, the differences and similarities between hosting and visiting friends versus relatives are underexplored. Lastly, the conceptualisation of the social interactions between immigrant-hosts and VFRs that encompass the temporal dimensions that the immigrant-hosts and VFRs go through as a process becomes a holistic approach and fundamental feature of this study.

As previously discussed in section 2.6, explaining the formulation of the conceptual framework, the tenets of social exchange theory are reciprocity, resource exchange, norms, and obligations (Cropanzano \& Mitchell, 2005). Empirical results show that social exchanges in the context of immigrant-hosts and VFRs are based on trust, reciprocity and commitment (explained further in section 6.4). However, given the contrast between friendship and familial relationships, the social exchange between friends is expressed differently as it is based on their past interactions and exchanges. Correspondingly, the social exchanges between and among family members is based on interdependence, care, and obligations or duties among the hosting and visiting family members. 
Figure 6.1. Reframing the immigrant-hosts and VFRs social interactions.

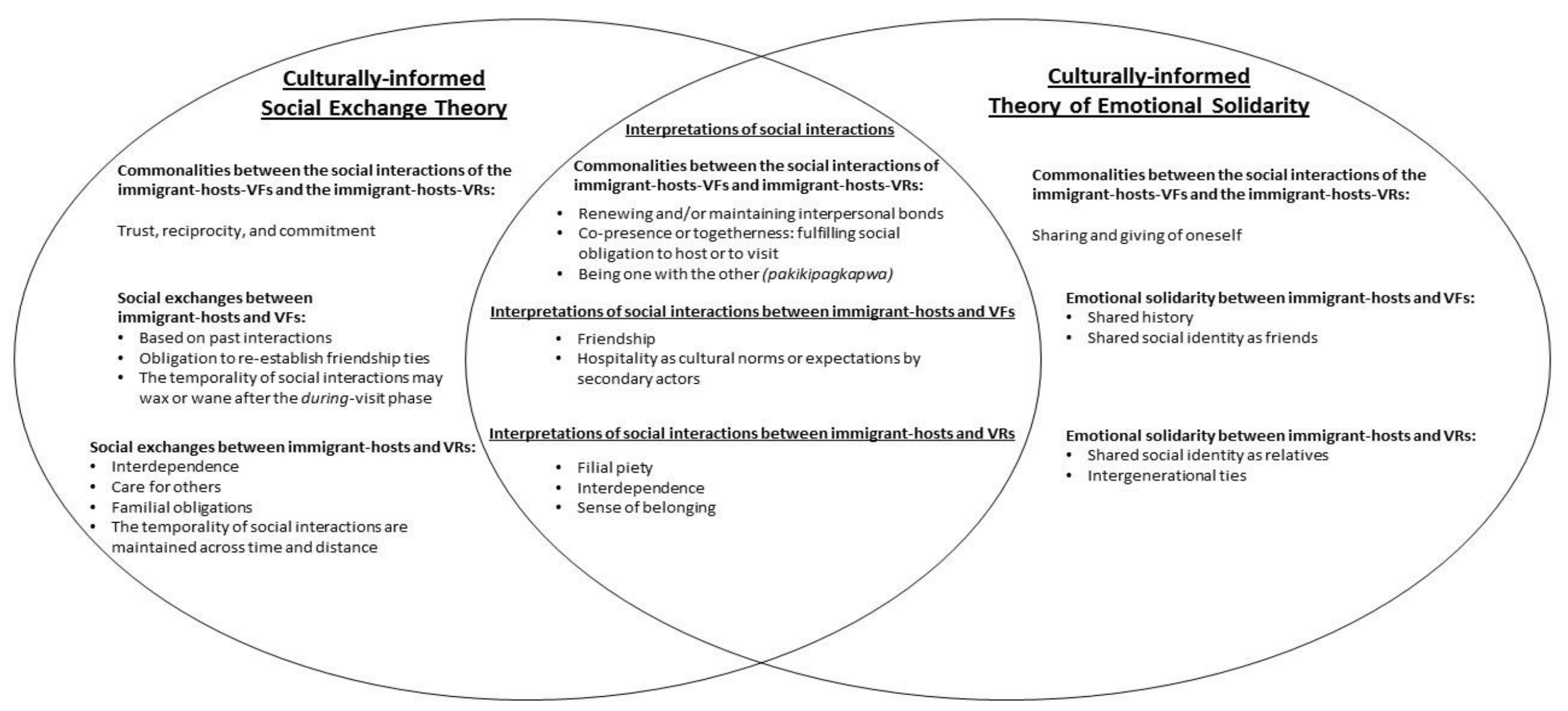


In contrast, the affective dimensions of the social interactions between immigranthosts and VFRs, using Durkheim's (1915/1995) theory of emotional solidarity, also reinforce the interpretation of their shared meanings. As previous studies have only focused on hosts and tourists, their understandings of the basis of their emotional solidarity were focused on their shared beliefs and shared behaviours. However, examining the conceptual lens in the context of VFR travel shows that emotional solidarity is also reflected in sharing and giving of oneself, regardless of being a host or guest (explained further in section 6.5).

The temporality of the social interactions between immigrant-hosts and VFRs has been examined encompassing the pre-, during-, and post-visit phases. There is much work yet to be done in analysing tourism in terms of travel phases (Fridgen, 1984; cited in Pearce, 2012) particularly between hosts and guests in the context of examining VFR travel. The temporality of social interactions that was developed earlier through the conceptual framework in Figure 2.3 provided the sequence for examining the social interactions of the immigrant-hosts and VFRs. However, as VFR travel is complex and dynamic, the social interactions reflect qualities that are described as "time-space compression" by Urry (2002b). This time-space compression is further expanded upon in the context of the social interactions between the hosts and guests in a VFR setting, where the social exchanges and emotional solidarity between and among friends and relatives are embodied in varying intensities and degrees over time and the exchanges between the hosts and VFRs are modified or transformed allowing social interactions to occur periodically even from a distance (e.g., by way of use of technology). For example, the findings of the study show that there can be significant variance in frequency of contact over time such that social interactions between immigrant-hosts and VFs which may wax or wane before and after the during-visit phase. Despite lulls in host-VF communication, the mutually voluntary nature of friendship may incite periodic future interactions that sporadically occur until the next visit takes place. In contrast, the dimensions of the social interactions between immigrant-hosts and VRs reflect on-going and persistent exchanges that are consistently and continually maintained across time and distance. This time-space compression that is occurring in a globalised world enables the fluidity of social interactions between the immigrant- 
hosts and VFRs that are therefore stochastic rather than static. Compared to the conceptual framework provided in Chapter 2 (section 2.6), the reconceptualisation of the temporality of the social interactions between hosts and guests within VFR travel are fused and integrated within the attributes of the mutual exchanges and emotional solidarity that may or may not be contemporaneous, but nonetheless these actors still adhere to the interpretation of what friendship and kinship means to them. However, the temporal and spatial sequence is a major challenge in capturing a holistic approach of the social interactions between hosts and guests. Despite this, a holistic approach has become one of the strengths of the thesis for examining social interactions across time and space in the context of VFR travel.

Contained within the overlapping portion of the ovals that reflects the social exchanges and emotional solidarity between the immigrant-hosts and their VFs or VRs are the interpretations of their social interactions which are at the heart of the conceptual framework. Since both friendships and familial relationships are principally concerned with sociability, the interpretation of their social interactions are commonly founded on renewing and/or maintaining interpersonal bonds, fulfilling social obligations through one's co-presence, and "being one with the other."

Even though commonalities were found in the interpretations of the social interactions between friends and families, each research participant had a unique experience that varied in some degree from all other participants. What primarily differentiates the social interactions between the hosts-VRs and the hosts-VFs is that the former social unit is influenced and characterized by family influences as the actors fulfil their roles of either host or guest but have additional influences of being related as relatives. In contrast, central to the social interactions between immigrant-hosts and VFs is friendship or hospitality where the particular roles of each actor within the VF exchange may have differing interpretations for each member of the group depending on the person's identity as a primary or secondary member of the hosting or visiting group. Such differences will be further illustrated and fleshed out in section 6.7, specifically the interpretion of the meanings of the social interactions between the participants within the groups of immigrant-hosts-VFs and the immigrant-hosts-VRs. 
However, the conceptualisation of the social interactions between immigrant-hosts and VFRs are not generalisable due to the small sample size and lack of representativeness. Specifically, this study examines the interpretations of the participants' social interactions and is uniquely focused on first-generation Filipino immigrants in New Zealand and their respective VFRs from the Philippines. While the study has a small sample size and therefore could lack representativeness, these qualitative accounts have produced an enriched cognitive schema that would enable an interesting way of examining the phenomenon (e.g., Donmoyer, 1990). This study also acknowledges its limitations such as an ethical inability to interview young children (discussed earlier in section 3.6: Strengths and limitations of the methodology) and the absence of multi-ethnic families from the sample (e.g., Filipino married to someone with a Maori or European background). Same-sex couples hosting for relatives could be the focus of a separate, stand-alone study (although this study includes an interview with a lesbian couple hosting a friend). Interactions between and amongst cousins either as hosts or guests - or between and amongst step relations could have been addressed as well. Since the majority of the social interactions between immigranthosts and VRs follows a matrilineal pattern as a principal characteristic of the phenomenon, the complexity of social interactions reflecting a patrilineal pattern of relationships between immigrant-host father/husband and his parents and relations as VRs were underexplored. Nevertheless, a small sample was selected precisely in order to reaffirm and convey that the intent of this hermeneutic phenomenological investigation which was to describe and uncover the personal meanings and understandings of the phenomenon, that is, the social interactions between the immigrant-hosts and VFRs.

In the qualitative sense, providing rich and thick description is a major strategy to ensure external validity (Merriam \& Associates, 2002). Such an interpretive paradigm aims to provide understanding of the social world, in this case, the social interactions from the viewpoint of the participants - the immigrant-hosts and their respective VFs or VRs, through detailed descriptions. Using hermeneutic phenomenology as a paradigm also means that the researcher is actively engaged in interpreting and 
understanding the social interactions between hosts and guests in the context of VFR travel while passing them along to the readers who may also reconstruct such interpretation differently (see also Lincoln \& Guba, 1985; Merriam \& Associates, 2002). Conceptualising and theorising about a group of people requires understanding how one's own perceptions of the world greatly influence what an individual thinks (Dilworth-Anderson et al., 2009, p. 634-635). The individual and group interviews with the immigrant-hosts and VFRs are interactions in themselves by which words are the main medium of exchange. It is not merely a one-way flow of information passing from the research participants to the researcher (Gaskell, 2003). The analysis and interpretations in this study are an exchange of ideas and meanings in which various realities and perceptions are explored and developed. As the research participants are providing their perspectives, the findings and interpretations are also influenced by this researcher whose life is also entangled within the Filipino diaspora through friends and relatives overseas (see also section 3.3: Self-evaluation and reflexivity; and section 3.6: Quality of interviews). A deeper understanding of the social interactions occurring between the hosts and guests will be further explored in the succeeding sections of this chapter and begins with section 6.3 which explores the nature of social interactions between immigrant-hosts and VFRs.

\subsection{Nature of social interactions between immigrant-hosts and VFRs}

In order to clearly address the first supplementary research question which asks, "What is the nature of the social interactions between immigrant-hosts and their VFRs?", there is a need to distinguish between the social interactions of the immigranthosts and VFs and those of the immigrant-hosts and VRs, which was previously suggested in the analytical framework in section 3.5. Several academic articles have examined the travel behaviours of VFs and VRs separately (e.g., Lockyer \& Ryan, 2007; Morrison, Hsieh, \& O'Leary, 1995; Moscardo et al., 2000; Navarro \& Turco, 1994; Seaton \& Tagg, 1995). However, the focus of these studies was mostly market oriented. A conceptual understanding of the social interactions between the immigrant-hosts and VFs and between the immigrant-hosts and VRs is a novel approach and significant contribution of this study. Given that studies of VFR travel are generally economically 
driven, they fail to address and recognise the complexity of interactions occurring between the hosts and their guests. Nonetheless, the previous studies mentioned earlier have provided direction and guidance for undertaking studies related to hostguest interactions through a sociological lens as social interaction is the most elementary unit of sociological analysis (Turner, 1988, p. 14).

Table 6.1 provides some dimensions used to highlight the nature of the social interactions between immigrant-hosts and VFs and between immigrant-hosts and VRs - including: (a) length of stay of the guest/s with the host family; (b) characteristics of the guest/s; (c) motivations of the host/s and/or guest/s; (d) return visit to the host/s; and (e) exchanges between the hosts and guests. Note that the parameters used are only those that emerged from the data and were used as baseline information within Chapters Four and Five. Other variables may also be included in future studies given that there is a current lack of empirical studies related to host-guest interactions within the context of the VFR travel literature.

Table 6.1. Nature of the social interactions between immigrant-hosts and VFs and immigrant-hosts and VRs.

\begin{tabular}{|c|c|c|}
\hline $\begin{array}{l}\text { Characteristics of } \\
\text { social interactions }\end{array}$ & $\begin{array}{l}\text { Immigrant-hosts and VFs } \\
\text { social interactions }\end{array}$ & $\begin{array}{l}\text { Immigrant-hosts and VRs } \\
\text { social interactions }\end{array}$ \\
\hline $\begin{array}{l}\text { Motivations of the } \\
\text { host and/or guest }\end{array}$ & $\begin{array}{c}\text { Diverse but obligation is } \\
\text { tied towards renewing } \\
\text { friendship or expressing } \\
\text { hospitality }\end{array}$ & $\begin{array}{l}\text { May be diverse but tied } \\
\text { towards obligation and/or } \\
\text { reunification with the family }\end{array}$ \\
\hline $\begin{array}{l}\text { Length of stay of the } \\
\text { guest/s with the host } \\
\text { family }\end{array}$ & Shorter & Longer \\
\hline $\begin{array}{l}\text { Characteristics of the } \\
\text { guest/s }\end{array}$ & $\begin{array}{c}\text { Itinerant and less } \\
\text { dependent on the host }\end{array}$ & $\begin{array}{l}\text { Interdependent and less } \\
\text { mobile }\end{array}$ \\
\hline $\begin{array}{l}\text { Exchanges between } \\
\text { the host/s and guest/s }\end{array}$ & $\begin{array}{l}\text { Occur intensely in the } \\
\text { during-visit phase but } \\
\text { tends to wax and wane } \\
\text { afterwards }\end{array}$ & $\begin{array}{c}\text { Generally continuous and } \\
\text { maintained across time and } \\
\text { distance }\end{array}$ \\
\hline Return visit to the host & $\begin{array}{l}\text { First time visitors and } \\
\text { therefore may require } \\
\text { longitudinal studies }\end{array}$ & $\begin{array}{l}\text { May be frequent depending } \\
\text { on the obligations within the } \\
\text { family }\end{array}$ \\
\hline
\end{tabular}


As the epistemological position of this study is based on hermeneutic phenomenology, the comparisons and contrasts between the nature of social interactions between these actors were only used as a form of classification which is a central process in conceptual understanding within the social science (see Bailey, 1994). In the same vein, the study does not intend to generalise the outcome of the research. Rather, the credibility of the qualitative results relies upon extrapolation by going beyond the narrow confines of the data and thinking about other applications of the findings. "Extrapolations are modest speculations on the likely applicability of findings to other situations under similar, but not identical conditions. It is logical, thoughtful, case derived, and problem oriented rather than statistical and probabilistic" (Patton, 2002, p. 584). The interpretations of the phenomenon were a result of extrapolation that is limited to the participants under study.

There is a lack of sociological understanding of the differences between interactions of friends and relatives, and there is a tendency to fuse both VF and VR travel into one group which is then commonly referred to as being as "VFR travel." For example, Moscardo et al. (2000, p. 251) speculates that if VFR is the sole purpose of the visit, there might be less interest in other tourist activities and the whole travel experience might be entirely focused on social obligations. However, this was not the case for the social interactions of immigrant-hosts and VFs within this study as the social interactions were more inclined towards the mutual enjoyment of touristic venues with friends. Both actors were engaged such that host families often become part of the touristic activities of the VFs or quite often may influence the choices of selected activities of VFs. Mutual enjoyment was often found to be the case based on the results in Chapter Four which focused on the immigrant-hosts and VFs and where the type of travel experience(s) depended on the social network that the host and/or guest maintains in New Zealand. In addition, the hosting of friends leans toward expressing hospitality as a way of sharing oneself where guests can probably derive pleasure from the visit considering that VFs are paying a somewhat shorter visit with the host family. Another factor that was discussed earlier is that VFs examined in the study were frequently first-time visitors to the immigrant-host's(s') new homeland, which was quite different from the case of VRs who are often frequent visitors to New Zealand. 
Perhaps VRs may have covered several touristic venues/activities during their previous visits with the immigrant-host family and are probably more focused on domestic oriented activities that promote family support, unity, and/or cohesion during their subsequent visits. Still, the social interactions between immigrant-hosts and VRs are more focused on everyday activities, where the latter becomes "temporarily" another member of the immigrant-host household.

The length of the visits of friends is shorter than the visits of family members and this dissimilarity corresponds to the differences of the intricacies of the relationships. Unlike friendship bonds which tend to result in visits that centre on an event or activity that usually focuses on one member of the host family with whom the VF has a significant past connection, family members generally visit to fulfil or carry out some familial obligation(s) such as providing child care, post-surgery recovery assistance, moral or spiritual support after the loss of a loved one, often for an indefinite or long period if necessary. In contrast, friends' trips are less structured by obligation and have a greater voluntaristic element (Lockyer \& Ryan, 2007) such that the visit usually occurs with the only goal of renewing friendship ties. As the responsibilities associated with friendship are generally considered to be more casual versus the sometimes heavy or enduring responsibilities that can occur between family members, hosting and visiting for friends accomplish the intended goals of the actors within a considerably shorter period of time.

In terms of the social interactions of VFs or VRs with their hosts, the VFs are frequently found to be itinerant while the VRs are mostly interdependent with their host families. Given the overall demographic profile of VFs in this study where they are characterised as young (and are more mobile), affluent, and maintain other personal relations in New Zealand, they have the opportunity to meet and spend time with friends as the visit is only one of the many activities of VFs. Such friendship networks often overlap with other people and often multiple visits occur on a single trip. However, host families for visiting friends may also influence the trips of their VFs and they often offer their network and resources to assist them in fully maximising the potential enjoyment of their friend's trip. This form of hospitality is embedded within the Filipino culture as 
understood by immigrant-host families (see section 4.6: Cultural beliefs and behaviours on hospitality). For VFs, the principal host within the family is obliged to offer hospitality while other members of the hosting family may also participate in varying degrees depending on their familiarity and past interactions with the guest. In this instance, secondary hosts (i.e., immigrant-host wives/mothers or children) who may not have a "strong" friendship bond with the VFs may accord the same hospitality being offered by the principal host as an expression of pakikipagkapwa or "being one" with their guests. Friendships are not just one-to-one ties, but may involve a number of people meeting collectively (or individually) for some purpose (Allan, 1989) as reflected in the social interactions between VFs and the hosting family (including secondary host/s members). As friendship is a voluntary relationship, the exchanges between friends occur such that one may make contact when they plan on visiting but communication between them may wax and wane as there may not a need to consistently and regularly communicate as their lives are not as multidimensional and intertwined as those of family members.

On the other hand, the VRs are interdependent with their hosts as there is a mutual exchange between the members of the host family and the $V R(s)$, which is maintained across time and space. Immigrant families, particularly the parents, may still be maintaining their familial obligations in the Philippines. However, during periodic absences of the immigrant parent from New Zealand, immigrant-host families may need the support and/or presence of a relative within their home in New Zealand. Therefore, the immigrant-host(s) are interdependent with their VRs and this is reflected in the nature of the social exchanges between and among the members of the hosting family and their relatives. The relationship between host parents in New Zealand and their relatives in the Philippines are very much intertwined with mutual obligations and expectations. Meanwhile, immigrant-host children may devote their time to their VR once the visit occurs, but may not have any specific obligation to them on a day to day basis other than periodic communication across distance and time, as that function is being maintained by their parents. 
As the relationships between immigrant-hosts and VRs are interdependent, there is a propensity for anticipated return visits to New Zealand which is not reflected in the social interactions between immigrant-hosts and VFs. Considering that the purpose of the visits of VRs is almost always tied to social obligation with the family, empirical results show that the majority of the VRs are return visitors and are involved in the different life phases of the immigrant-host family (e.g., from the birth of the children of the immigrant-host wife/mother, the baptism of the child(ren), birthday celebrations within the hosting household or the VR her/himself, and deaths in the family). VFs are not necessarily obliged to make themselves available to assist their immigrant-host friend(s) in a time of need. Currently, there are no statistical data or existing studies available that separately details or compares the return visits of VFs to those of VRs. However, there are other interpretations of friendships that were not included in this study, such as romantic relationships being maintained across distance. It may be assumed that return visits for other types of friendship such as for romantic relationships are also done frequently.

As social interaction is symbolic (Nash \& Calonico, 1996) and performed through the hosting and visiting friends or relatives, the motivations of the different members of the hosting family and their respective VF or VR are arbitrary and intersubjective. Within the mobilities paradigm, there is a complex relationality of places and persons connected through the performances (Sheller \& Urry, 2006) in which first-generation Filipino immigrants and their VFs or VRs are embedded. While this study provides a holistic approach to investigating the motivations of the hosts and guests, these actors construct different interpretations with regard to their motivation(s) to interact with their guest or host. The reconceptualisation of VFR travel should offer a more holistic view to include any tourism-related experience that involves a prior personal relationship (Griffin, 2013) between hosts and guests which is further elaborated in section 6.4 as it deals with the social exchanges between immigrant-hosts and VFRs. 


\subsection{Social exchanges between immigrant-hosts and VFRs: trust, reciprocity, and commitment}

The use of social exchange theory in this section to interpret the social interactions that occur between hosts and guests will address the second supplementary research question "What are the social exchanges between the immigrant-hosts and their VFRs?" Several studies have examined host-guest interactions through the theoretical lens of social exchange (e.g., Ap, 1992; Byrd, Bosley, \& Dronberger, 2009; Fredline, 2005; Hernandez, Cohen, \& Garcia, 1996; Moyle et al., 2010; Zhang, Inbakaran, \& Jackson, 2006). While these studies have provided insights into the social exchanges of host-guest interactions, the typical interpretation in tourism studies has either been treated at the macro-level (Dann \& Cohen, 1991) through a utilitarian calculation of costs and benefits or with a focus on exchanges between "self" and "others."

In contrast, the findings of this thesis show that VFR travel at the micro-level is a complex dynamic of host families and their VFs or VRs who are familiar with their relationships. It illuminates the range of perspectives of families and friends with regard to their exchanges in order to enhance understanding of the interactions between immigrant-hosts and VFRs. Since VFR travel is multi-faceted, involving both the hosts and guests, examining the exchanges between immigrant-hosts and VFRs across time and space provides a holistic approach for viewing social interactions that are experienced by them. Usually, the temporal dimensions of experiences are focused on the individual and typically on the tourists (e.g., Cutler \& Carmichael, 2010; Gyimóthy, 1999).

However, this study reveals that VFR travel as a phenomenon is multi-faceted involving families and friends who are interacting across time and distance. As an example, obligations are not fixed in time and space, but are negotiated, contested, and enforced (Larsen et al., 2007) and this is also true with hosting and visiting for friends and relatives. The exchange of favours or choices of activities are negotiated, even among family and friends, within an interaction. Still, this form of social relationship is characterized by both the reciprocal exchange and the expectation of continued interaction, which are particularly conducive to building trust (Molm, Takahashi, \& 
Peterson, 2000). Therefore, while the nature of the social exchanges between families and friends are separate and distinct, these social interactions are generally based on trust and within the tenets of reciprocal exchange. As Molm et al. (2000, p. 1423) further elaborated: "In reciprocal exchanges, actors choose individually to give to one another, without any formal assurance of reciprocity. No matter how established the relation, how predictable the other's behaviour, and how long the "shadow of the future", each act of giving still remains a declaration of trust that the other will reciprocate, and each act of reciprocity confirms that trust."

Earlier, the social exchanges between friends in the context of immigrant-host and VF were discussed within the tourism literature with a focus on their past interactions (discussed in section 4.4). The during-visit phase between friends allows them to reestablish their social exchanges after being separated by time and distance. However, it also allows the compressing of time and space where friends re-acquaint themselves with one another in order to renew their friendship ties. Mutual liking, shared experiences, care and trust are the core elements of friendship (Annis, 1987) and the visit provides the critical time that is necessary to renew the friendship bonds and to create new memories that allows the relationship to move forward. These periods of renewal of ties are all manifested in the relationship between the immigrant-hosts and VFs where the majority of friendships were established long before the migration of the host families.

Friendship cannot be coerced, even if external forces were to influence the interaction between persons (Dreher, 2012). The hosting of friends by immigrant-hosts is an invitation to a type of intimacy which is an offering to share in the host's private life (see O'Gorman, 2010; Telfer, 1996). In this case, the act of hosting gives meaning to the friendship with their guests by the giving of oneself in a manner which is not offered to a stranger and even secondary members of the host family are involved in the hosting by the giving of trust to their guest. In other words, we expect less empathy from a common acquaintance than from a friend; we expect still less empathy from an assembly of strangers (Smith, 1976). On the other hand, there is also a strong 
commitment on the part of VFs to renew their ties with their friends despite having multiple motivations for visiting New Zealand.

Similarly, the social exchanges between relatives are based on the interdependence (elaborated on earlier in section 5.3.1) of the Filipino immigrant-host families in New Zealand with their kin in the Philippines and vice versa. This type of relationship assumes there is a trusting environment within the family or among relatives as there is mutual commitment to help one another. Maintaining ties across the distance for immigrants and their respective relatives is manifested in the ongoing exchanges between members of the hosting family with their guests by providing assistance or care and other social or familial obligations which is a reciprocal relationship in itself. For example, the parent-child bond between the visiting parents/grandparents and immigrant-host mother/wife is continued in New Zealand as VRs often assist their respective immigrant-host families, whether in terms of performing traditional or domestic duties (e.g., childcare), performing property maintenance or simply by reaching out to bond with the immigrant-host children/grandchildren.

There is also a chain of exchanges occurring between VRs and the immigrant-hosts. In New Zealand, VRs may become important social capital for immigrant-host families in the form of a commitment to the provision of support to immigrant families. However, while the VRs may also be supporting their relatives, they are motivated by other familial obligations which exist in the Philippines. The fact that VRs from the Philippines stay longer with their host families in New Zealand to assist them is a social exchange that is founded on their commitment to look after the wellbeing of their relatives. However, the social exchange is mutually beneficial and the VRs, through their acts of benevolence during the visit are also strengthening the likelihood that they can expect support in a time of need from their immigrant-hosts. After this discussion on the scope of social exchange theory in the context of immigrant-host's(s') and VFR's(s') social interactions, the next section will further expand the interpretation to include the affective aspect of their social interactions through the theory of emotional solidarity. 


\subsection{Emotional solidarity between immigrant-hosts and VFRs: sharing and giving of oneself}

This section provides interpretation of the social interactions between hosts and guests through the lens of the theory of emotional solidary by addressing the third research supplementary question "How do social interactions reflect emotional solidarity between the immigrant-hosts and VFRs as expressed by their shared beliefs and behaviours?" Unlike social exchange theory, the theory of emotional solidarity is an underexplored concept in the field of tourism research. While recent studies have examined the theory of emotional solidarity (e.g., Woosnam, 2011; Woosnam \& Aleshinloye, 2012; Woosnam \& Norman, 2009; Woosnam et al., 2009), those studies were focused on the resident-tourist interactions.

Since the focus of earlier studies are centred on the "self" and "others", the conceptualisation of the social interactions between these actors using the theory of emotional solidarity has only captured their shared beliefs and/or behaviours. However, the emotional solidarity demonstrated between and among friends and families through VFR travel stems from sharing and giving of oneself. Thus, the social interactions between immigrant-hosts and VFRs is an endeavour to make sense of their relationship with the "other" through the shared experience (see Hibbert, Dickinson, \& Curtin, 2013). Hosting and visiting friends or relatives as a form of social interaction allows the bodily co-presence of people in a specific place and time providing moments for physical proximity between hosts and guests that probably makes the visit desirable or even obligatory for some (see Germann Molz, 2006; Hannam, Sheller, \& Urry, 2006; Urry, 2003).

As the social interactions of the immigrant-hosts and VFs are rooted in their past interactions (discussed in detail in section 4.4), it is assumed that the shared history, at least, between the primary host/s and the primary VF/s has overall produced positive emotion based on positive evaluation of the outcomes of exchanges between them. The shared history between them allows a continuation of their friendship which allows them to maintain their relationship from a distance. Despite limited contact or irregular communication, the ties of friendship have persisted over the years despite 
extended periods of separation and have endured up to the time of the study and will probably be maintained over a long-term period through the shared recollection of experiences and which are probably enhanced from the opportunity to experience situations in the co-presence of the other during the occasion of a visit. However, to determine if the involvement of secondary hosts or guests in future exchanges would require further investigation. Nonetheless, these secondary actors (e.g., the immigrant-host son in the Mayon family; Mara in the Taal family; and the visiting mother of the Ragang family's best friend) still express their sense of sympathetic fusion or oneness (pakikiisa) with the rest of the members involved in the social interaction by expressing their solidarity in the activities involving the primary host/s and $\mathrm{VF} / \mathrm{s}$.

On the other hand, the social exchanges between immigrant-hosts and VRs discussed earlier (in section 6.4) show interdependence between and among these actors may also generate a sense of "groupness" among actors (Lawler et al., 2000). In the context of solidarity within families, several authors in social psychology (see Bengtson \& Schrader, 1982; Roberts, Richards, \& Bengtson, 1991; Silverstein \& Bengtson, 1997) have identified six dimensions underpinning intergenerational solidarity: (1) structure (factors such as geographic distance that constrain or enhance interaction between family members); (2) association (frequency of social contact and shared activities between family members); (3) affect (feelings of emotional closeness, affirmation, and intimacy between family members); (4) consensus (actual or perceived agreement in opinions, values, and lifestyles between family members); (5) function (exchanges of instrumental and financial assistance and support between family members); and (6) norms (strength of obligation felt toward other family members).

Using these six dimensions, the social interactions between immigrant families and their VRs indicate that the social interactions are still being maintained across the distance (structure). In terms of association, immigrant families also share activities as VRs become part of the shared common experiences described as the "extraordinariness in the everyday life" of the hosts. With regard to affect, the filial obligation to assist VRs in the Philippines, at least, is still being shared by immigrant- 
host parents as a cultural value. For immigrant-host children (or grandchildren) who did not grow up with their VRs (mostly visiting grandparents or aunties), sharing oneself is shown by observing and adhering to the cultural values of their parents' former homeland in order to avoid conflict with their VRs (described in section 5.5.4: Barriers to familial solidarity). Consensus, functions and norms and other dimensions of intergenerational solidarity within the family have been discussed previously in Chapter 5, where there is an ongoing mutual exchange and assistance within the family such as child care, attending social obligations, or even assisting a distant relative seeking employment. Hosting and visiting families in and of itself is an authentic expression of their shared identities as being family members and they share themselves by "being together" within a shared life experience in New Zealand.

This study contributes to further knowledge in utilising the theory of emotional solidarity to interpret the host-guest interactions in the context of VFR travel. Hannam et al. (2006) suggest that social research should be more attentive to researching the affective dimension of both actors and their performances. Using Durkheim's theory of emotional solidarity in the conceptualisation of the host-guest interactions, the ultimate motivating force in human interaction is the need to affirm group membership and a sense of solidarity with others, leading actors to initiate and sustain interaction rituals (Turner, 1988, p. 38). The emotions that individuals experience as a result of social interactions (in this case, the hosting and visiting of friends and relatives) are likely to be perceived as jointly produced. This interaction or exchange has emotional effects on individual actors and those emotions affect the strength of their group affiliations or attachments (Lawler \& Thye, 2006, p. 301). Through VFR travel, hosting and visiting as a social obligation between friends or family is essential for developing the relations of trust (an important consideration in social exchange discussed in section 6.4) that persist during often lengthy periods of distance and even solitude (Urry, 2003). After interpreting the social interactions between hosts and guests through social exchange theory and the theory of emotional solidarity, the next section will discuss how culture constructs the social interactions between the immigranthosts and VFRs. 


\subsection{Cultural influences in the social interactions between immigrant- hosts and VFRs}

This section addresses the last supplementary research question: "How does culture shape social interactions between the immigrant-hosts and VFRs?"In this study, culture is represented by the symbols, meanings, and values that are shared by members of the group and is identifiable by the nature and types of social interactions in which they engage during the hosting for and the visiting of friends and families. Reisinger and Turner (2003) stated that all interactions are viewed to a certain extent as "intercultural," and the degree of their "interculturalness" depends upon the degree of heterogeneity between cultural backgrounds of the individuals involved in interactions and also upon their patterns of beliefs, verbal and non-verbal behaviour, perceptions and attitudes.

As a symbolic representation of culture, the social interactions between firstgeneration Filipino immigrants hosts and their respective VFs reflect the "interculturalness" through their shared views of hospitality (discussed in Chapter 4, section 4.6). The perceptions and experiences of the hosts and VFs reflect their respective notions of hospitality in terms of cultural norms and expectations of hosting and visiting. Within the context of Filipino culture, VFs and hosts have expectations of the kind of hospitality that will be offered to visiting friends which is based upon Filipino cultural norms and their specific shared past experiences and the resulting visiting and hosting experiences are the product of their "interculturalness." In reality, this may not be the case when the same VFs approach "others" to host for them, particularly those outside their "in-groups" and with whom they do not have particularly strong personal ties due to a perceived lack of shared past experiences. Nonetheless, the act of hosting by the immigrant-hosts minimizes any "strangeness" that may be felt as they open their home and provide hospitality to their VFs.

In philosophical terms, Snow (2010, p. 16) views hospitality as a virtue that is: "construed narrowly as making others welcome in one's own life and world; and construed more expansively as making others welcome in a shared life and world." Underpinning feminism and hospitality, Snow elaborates these two separate 
arguments by differentiating the former where hospitality means choosing to share one's rightful possession with others. However, the latter part of the discourse defines hospitality not as the sharing of one's possession but the sharing of one's life. Rather, hospitality as a value means making available to others what is already shared among them. In this case, the act of hospitality being expressed is the friendships being shared by people within a social interaction. The friendship that is therefore shared by immigrant-hosts and VFs is linked to the social exchanges and emotional solidarity (discussed earlier in sections 6.3 and 6.4 of this chapter) that results in the mutual trust and commitment and the affective dimensions of sharing and giving of oneself between friends that is performed and continued through each other's co-presence in the immigrant-host's(s') new homeland. Although friendships differ from culture to culture, it is possible to describe recurrent practices such as mutual aid between friends based on need, mutual affect and gift-giving between the parties involved (Dreher, 2012).

Further, the hospitality that is expressed in welcoming someone in a shared life is anchored in the Filipino inter-personal relationship of pakikipagkapwa (mutual trust). It entails empathy which is grounded in one's ability to imagine what it would be like to be in the other's shoes, as pakikipagkapwa fosters sensitivity to the "otherness of the other" (Guevara, 2005). While there are differences between and among friends (e.g., beliefs, behaviours, interests, values, or status), it is within the context of the essential differences that immigrant-hosts and VFs (the primary host/guest, in particular) come to understand "shared identity" as sharing the universal experience of friendship (i.e., the past interactions and the shared history that they enjoyed before the immigrant-host migrated to their new homeland). Friendships may even be strengthened by tapping into other friendship networks existing within one's culture, such as through co-parenthood ("god parenting") in the Philippines (e.g., Kikuchi, 2001) and also known as "compadrazgo" system in Latin American countries (e.g., Gomez \& Rodriguez, 2006) where mechanisms of solidarity and reciprocity are developed in order to minimise uncertainty through the help of others. 
Despite being in solidarity for the during-visit phase, the secondary member(s) of the hosting or visiting party may not necessarily have the benefit of a shared universal experience of friendship that exists between the primary host and guest. As this study has examined the perspectives of multiple hosts and guests, secondary members of both the hosting household and visiting parties have been involved and expressed their views. As a consequence, a gendered dimension of the interactions has been illuminated. In particular, while friendships between hosts and VFs may be heterogeneous, e.g., involving same sex or opposite sex friendships, immigrant-host wives are greatly involved in hosting for their guest(s) even if they are not the primary host. An example of this among the secondary hosts interviewed would be the immigrant-host wife for the Halcon family who felt some stress due to her lack of familiarity with her new homeland. A study by Sobritchea $(2012$, p. 31) in relation to the female domesticity of Filipino women shows that while husbands form and maintain friendships (usually with males) outside the home, the wife is more likely to associate with her own relatives (i.e., parents and siblings) before friends (usually female) in that order. However, her family and husband remain first and foremost in her preferences for the sharing of her time. Future studies may develop this gendered perspective further reflecting the socialisation between hosts and guests from other cultures in the context of VF travel.

This study discloses the cultural representation of immigrant-host and VR social interaction reflected in the intergenerational ties that are being continued by families and unites the relatives in the Philippines and their respective host families in New Zealand. Evidence from this study shows that the ties of the relationship between hosts and guests follow a matrilineal pattern which means that the principal host within immigrant-host families is normally the mother or wife (previously discussed in sections 3.4.3, 5.2.1, and 5.6). Drawing on the social structure of families, members of every human society belong to a family of orientation by which a person may be born and/or reared which includes one's parents and siblings. ${ }^{18}$ On the other hand, the

\footnotetext{
${ }^{18}$ The author acknowledges that a family of orientation can include a person who may be legally adopted by a family or one's adoptive parents. A person may also be raised by extended family members (e.g., grandparents; uncle/auntie) or foster parents in the absence of biological parents.
} 
family of procreation ${ }^{19}$ which one establishes by marriage and which includes a couple (husband and wife) and the children were discussed earlier in section 2.5. The terminologies used in this study represent these two types of families. For example, the female hosts are mothers to their own children in New Zealand (or wives to their husbands) but also are daughters to their parents who come as guests (VRs). This means that the intergenerational ties that act as a catalyst to VR travel largely occur due to immigrant-host mother/wife (see Figure 6.2). Note also that the family of orientation and the family of procreation represents two types of families that are geographically separated, in this case, one being in the Philippines and the other in New Zealand, respectively.

Figure 6.2. Illustrative representation of the intergenerational ties between two families in the context of VR travel.

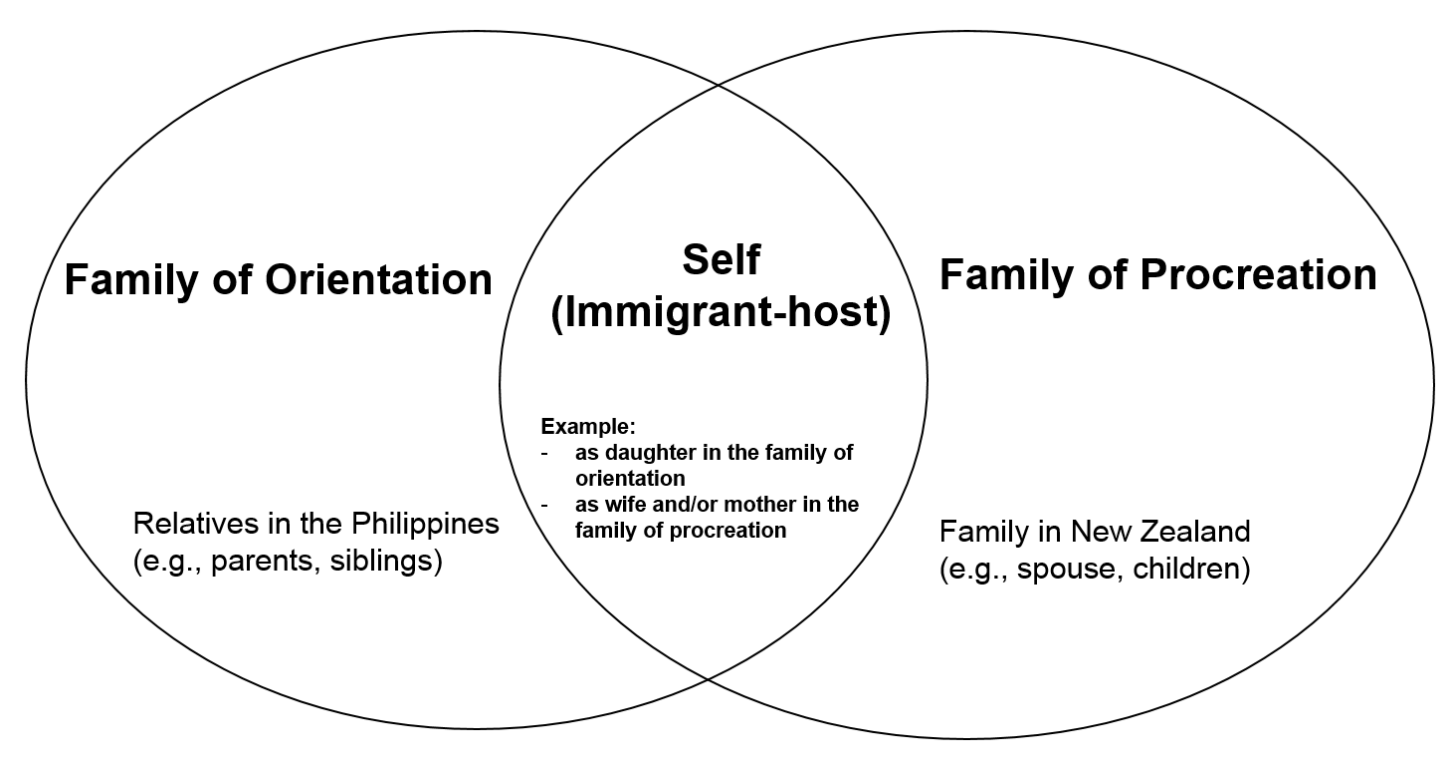

The matrilineal pattern found in the social interactions between first-generation Filipino immigrants and their VRs is best described through the Western adage: " $A$ daughter is a daughter all of her life, but a son is a son "til he takes him a wife" (cited in Merrill, 2011). Within this framework, VR travel occurs due to gendered and cultural expectations within the two types of families (the family of orientation and the family

\footnotetext{
${ }^{19}$ Today's families are diverse and may include other types of families (e.g., same sex couples) who are also capable of raising a child. In the Philippines, however, gay or lesbian couples are barred from legally having a joint adoption based on the 1987 Family Code of the Philippines.
} 
of procreation). There are three cases that would explain the phenomenon surrounding the social interactions between immigrant-hosts and VRs. First, there is interdependence between the immigrant-host wife/daughter with her family of orientation. Second, there is transplanting of the motherhood roles of Filipino women in New Zealand (detailed in section 5.5.3). Lastly, there are ongoing exchanges between the immigrant-hosts and VRs influenced by the cultural value of filial piety (discussed in detail in section 5.5.1).

The interdependence between immigrant-host mothers and their relatives in the Philippines evolves because of her efforts to incorporate her family of procreation in New Zealand into her family of orientation (and this may even be extended to other relatives such as an auntie). This is also evident in the study of Silverstein and Bengtson (1997) where daughters serve as significant elements in the kin matrix and this feminine influence on family bonds is based on their biosocial mechanism of early socialisation until the adult life course. In addition, women place greater value on the personal relationships (Merrill, 2011) which was made apparent in this study when immigrant-host wives would socialise with their VRs regardless if they belong to her family of orientation or not. Also, these immigrant-host wives will put a premium on the relationships and this is expressed in her efforts and dealings when hosting for her husband's family of orientation and/or extended relatives in the Philippines. However, the phenomenon under study was solely based on the latest hosting experience of the Filipino immigrant families and did not include the previous social interactions with other VRs.

Since most of the immigrant-host families in the study are young families who are struggling to raise their children in their new homeland, it is the immigrant-host mother/wife who carries a multiplicity of burdens such as fulfilling her obligations within the family in terms of domestic duties (reproductive roles) in the home and in contributing within the economic sphere (productive roles) outside of the home. These diverse familial obligations are therefore the traditional roles that women carry or have transplanted with them when the family migrated. Hence, interdependence occurs between the immigrant-host wives with their VRs as she may be needing assistance 
within her family of orientation at a time of need or crisis within the family, e.g., postnatal care, child care, illness which may require that VRs stay longer with the immigrant-host family. In contrast, while the family of orientation of the immigranthost husband/father may have previously visited New Zealand, they do not visit nearly as long as compared with the family of orientation of the wife/mother and was noted when the immigrant-hosts were recalling the previous visits of other relatives from the immigrant-host husband's family of orientation. This difference has apparently been echoed by the immigrant-host mothers/wives in terms of seeking assistance within child care or post-natal care from her family of orientation in the Philippines (see section 5.5.2: Notions of caring for Filipinos) when dealing with her requests for assistance with her domestic responsibilities in New Zealand.

In addition, as immigrant-host wives/mothers maintain their interpersonal relationship with their VRs in the Philippines, the ongoing exchanges are founded on honouring of the cultural value of filial piety which is recognised by way of caring for her relatives in the Philippines as well as those of the immigrant-host husbands/fathers. The economic support being provided by the immigrant-host families to their VRs may also be shared by the latter to help other family members in the Philippines. This reciprocity between the immigrant-host family (particularly the mother/wife) and their VRs is treated like an obligation and symbolizes a push and pull factor for the two types of families to continue their relationships.

Apparently, the identity of Filipino immigrant-host fathers is also transplanted in New Zealand. McKay's (2011) research shows that the dominant and central element concerning Filipino fathers is being a good provider for the family as they are considered the "pillar of the home" (or haligi ng tahanan in Filipino terms). This is manifested in how immigrant-host fathers/husbands view hospitality by being a provider of material goods to their guests (see section 5.4.1: Hospitality and sociability between immigrant-hosts and VRs). Additionally, immigrant-host fathers/husbands take pride when they can show their VRs (usually parents-in-law) that his family of procreation is achieving a better quality of life in New Zealand compared with their social or economic status in the Philippines (see also section 5.6: Meanings of the visit 
for relatives - in particular, examining the statements of immigrant-host fathers/husbands), and is a reflection of what a father is expected to be within the Filipino culture and society.

Considering how the culture and residency shapes the social interactions among the immigrant-host children, cultural differences between them and their respective VRs may become a hindrance in their socialising. In some instances, it may produce tension or misunderstanding as these children may no longer share the same Filipino cultural values which their guests possess (see section 5.5.4: Barriers to familial solidarity). As these immigrant-host children have grown up in a different cultural setting, they may acquire traits of self-reliance and independence which allows them to display more autonomy and encourages them to explore their environment (Triandis, 1989) that is different from their VRs who may emphasise the self in relation to others or the self in relation to the world. However, individualism and collectivism as cultural or behavioural constructs (see Mesquita, 2001) may not be sufficient in interpreting the social interactions between the immigrant-host children and their guests. The conflict that often emerges between the immigrant-host children and their VRs is shaped by their cultural identity as Filipinos which they share with their parents and their emerging identity as New Zealanders. Wolf (1997) argues that assimilation may not be sufficient to describe the depth of complexities that children of immigrants are confronting. The author conceptualises that emotional transnationalism evokes more of a sense of multiple discourses circulating and competing in the lives of the children of immigrants, which transcends the binary and segmented notions of acculturation or assimilation. This transnational approach acknowledges multiple locations of "home" which may exist geographically, ideologically, and emotionally, in addition to a plurality of cultural codes and symbols. Hence, the cultural symbol of hosting for Filipino relatives may not be completely understood by these children as compared to their parents' appreciation, especially when they have to give up their bedroom or privacy, wake up early in the morning to have breakfast together with their parents and their VRs (usually grandparents), or make other sacrifices in order to accommodate their visiting relatives. Nonetheless, immigrant-host children (particularly those who were raised in New Zealand) acknowledge that time is an essential element when hosting for 
their relatives, of which they may not have been able to provide completely due to other commitments in their life.

Indeed, understanding the world-view of hosts and guests is necessary to grasp a culture's most significant values, and thus how and why members of a culture behave as they do (e.g., Watkins \& Gnoth, 2011). This has been revealed in examining how culture shapes the social interactions between immigrant-hosts and VRs that shows the interdependence that exists between the immigrant-host families with their relatives in the Philippines. Regardless of the culture, friendship as a universal experience for immigrant-hosts and VFs enables the hosting and visiting of friends. The next section will now address the main research question of this thesis in relation to how social interactions hold important meaning for the hosts and guests.

\subsection{Interpretations of social interactions between immigrant-hosts and VFRs}

This section brings together the supplementary research questions of this thesis by addressing the main research problem: "How are social interactions between immigrant-hosts and their visiting friends and relatives understood and interpreted by them?" The interpretation and understanding of social interactions, which form the basis of this study, depends on the hosts and guests remembering their experiences and encounters with one another. Given that hermeneutic phenomenology is the underpinning paradigm for this study, Pernecky and Jamal (2010) pointed out that "truth" is neither an objective endeavour nor something awaiting "verification" or "confirmation." It is an interpretive construct and involves assessing the trustworthiness or credibility of the researcher's interpretation of the participant's experiences (as described by the participants). As this Filipino researcher also experiences a traditional family life that is entangled with relatives and friends who were overseas and was eventually educated in Anglo-American institutions, this set of experiences and influences would be reflected in the interpretation of the host-guest interactions, a recognition that such an approach is not without its limits (see also section 3.3: Self-evaluation and reflexivity). 
Social interaction is materially organised, whether the proximities involved are "bodily", "virtual”, or "imaginative" ( Bǣrenholdt, Haldrup, Larsen, \& Urry, 2004, p. 33). Traditional research in VFR travel has only specifically focused on either the host or the guest as separate units and this has had significant implications for the lack of understanding of the motivations of these actors. Understanding host-guest social interactions as a social phenomenon involves subjective meanings where this expression is situated in some context within a social world by which we must know its location, who speaks, who knows what, who is trying to get something done, or what that something is (see Nash \& Calonico, 1996).

This section begins by examining the social interactions between friends (immigranthosts and VFs) followed by families (immigrant-hosts and VRs). When examining a relationship which Ho (1998) defines as a particular type of connection existing between people related to or having dealings with each other (i.e., as friend or as relatives), the previously cited author demonstrates that the strategic units of analysis are not the individual or the situation alone, but "persons-in relations" (focusing on a person in different relational contexts) and "persons-in-relation" (focusing on persons interacting within a relational context). Also, the spatial and temporal specificity of the social interactions between the immigrant-hosts and VFRs has significant implications for understanding their experiences and for enhancing their relationships through social interactions.

The first illustration depicts the interpretations of the social interactions between immigrant-hosts and VFs (Figure 6.3) as interpreted by the primary and secondary actors. On one hand, the underpinning dimension of the social interactions between the immigrant-hosts and VFs is that of friendship, particularly for the primary actors (Figure 6.3a). At the heart of the social interactions between VFs and the immigranthost family is that friendship is a voluntary relationship (refer to the literature review in section 2.4: Friendships, social interactions, and VF travel research). On the other hand, Figure $6.3 \mathrm{~b}$ pertains to the interpretation of the social interactions between the secondary members of the hosting and visiting group that is centred on hospitality. 
Figure 6.3. Illustrative representation of the interpretations of the social interactions between the immigrant-hosts and VFs.
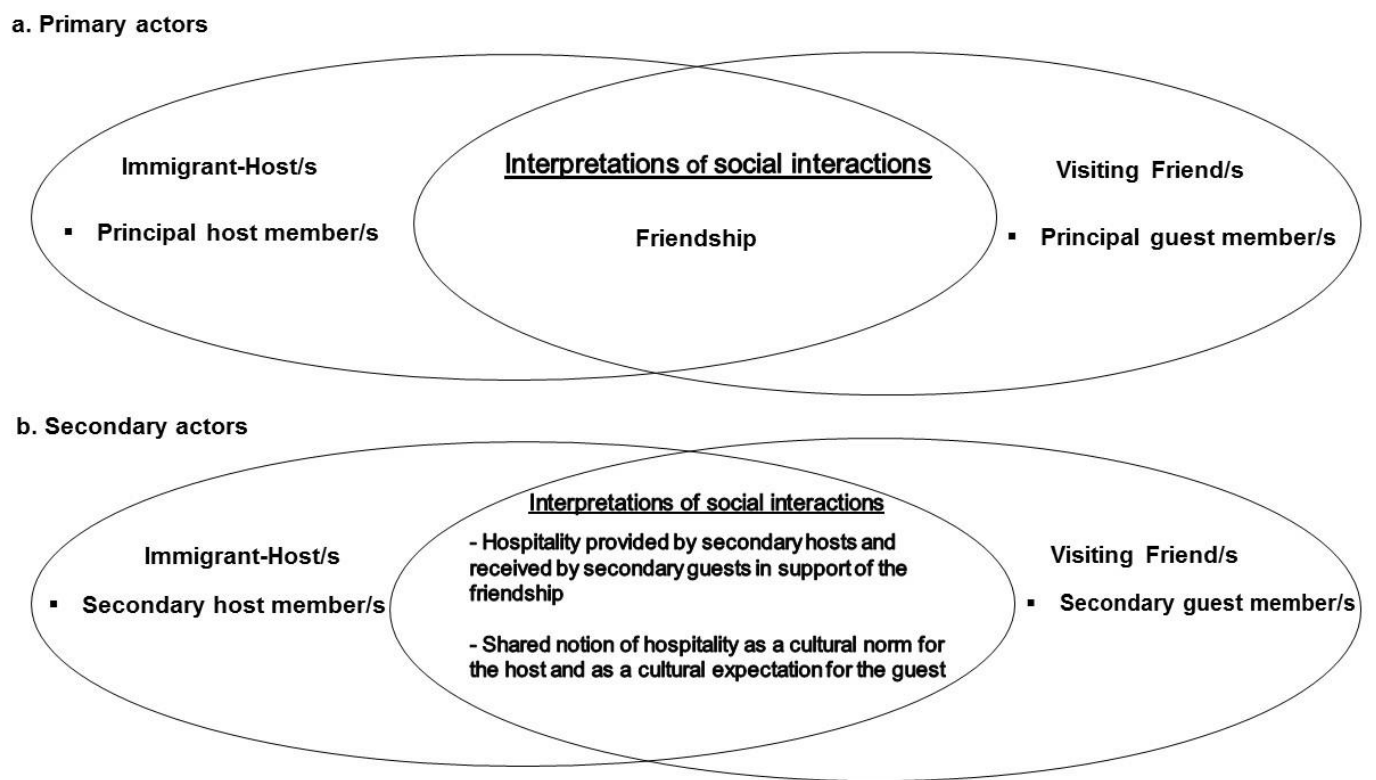

While the primary and secondary actors within the hosting and visiting group may be in solidarity in the during-visit phase, what defines the primary actors of a friendship (in Figure 6.3a) is that hospitality is expressed because they have a shared or harmonious interpretation of their relationship. This is based on the immigranthost's(s') and VF's(s') past interactions (see also section 4.4) and that friendships are fostered and re-established through the co-presence of the other. VF travel has a rituallike quality as intimacy and trust is vital in the maintaintenance of friendship ties despite infrequent or irregular communication across time and space that can result when one participant in the relationship moves far away. The social interactions between friends generates a relational effect by encountering the different facets of one's personal self (see Pons, 2003) that can be achieved through the bodily copresence of friends, who happen to be in that place at that time and doing things together during a visit. Compared with acquaintances, it is with close friends that one may discuss the subjects closest to one's heart which may be sacred to a person (Wallace \& Hartley, 1988).

Meanwhile, social interactions occur among the secondary members of the hosting and visiting group in Figure 6.3b and are based on their shared notion of hospitality being 
auxiliary members in the interactions. What differentiates them from the primary actors is that they are not central to the friendships under examination (based on the secondary host's(s') possible lack of past interactions or lack of social exchanges with the VFs which was discussed in section 4.4). Secondary hosts and secondary guests provide and receive hospitality, respectively, through their association with the friendships of the primary hosts and guests. The interpretation of the social interactions for the secondary actors is influenced by the cultural norms of hosting for guests, while the VFs are guided by their cultural expectations of hospitality when being hosted. Although these secondary actors have to make their behaviour understandable to others (Goffman, 1967) in order to convey the interpretation of hospitality, they may not necessarily share a similar view of the significance of the friendship that the principal hosts and VFs share but the secondary actors are however generally sympathetic to the primary actors and are supportive of their interaction.

The second illustration (Figure 6.4) depicts the three underlying dimensions of VR travel in the context of the social interactions between immigrant-hosts and VRs. These are: (1) filial piety; (2) interdependence; and (3) sense of belonging (see Figure 6.4). The illustration was intentionally used as a representation of these two different spatial dimensions: the Philippines and New Zealand, and this figure can also be used to illustrate different relationships (e.g., between siblings or in-laws; parent-child; grandparent-grandchild).

In relation to the first dimension of the interpretation of social interactions, filial piety is an important and predominant cultural value in East Asia that is still understood by both the immigrant-host parents in New Zealand and the VRs (the grandparents) in the Philippines (as discussed in detail in section 5.5.1: Filial piety). However, as immigranthost families move to New Zealand, the social structure of the family suddenly departs from the traditional or extended family that predominantly exists in a traditional society and immediately upon emigration becomes a nuclear family (two parents, male and female, and children) and the relationships experienced by the family may not be as oriented towards other extended family members left behind (i.e., grandparents, aunties, uncles, and cousins) as they once were. Therefore, filial piety may no longer 
be shared or recognised by the immigrant-host children as it only deals with parentchild and spousal relationships which contrasts with the family experience in the Philippines where, for example, immigrant-host husbands/fathers may on a daily basis treat their in-laws the way their wives (the immigrant-host mothers) treat them as an expression of mutual trust (pakikipagpalagayang-loob).

Figure 6.4. Illustrative representation of the interpretations of the social interactions between immigrant-hosts and VRs.

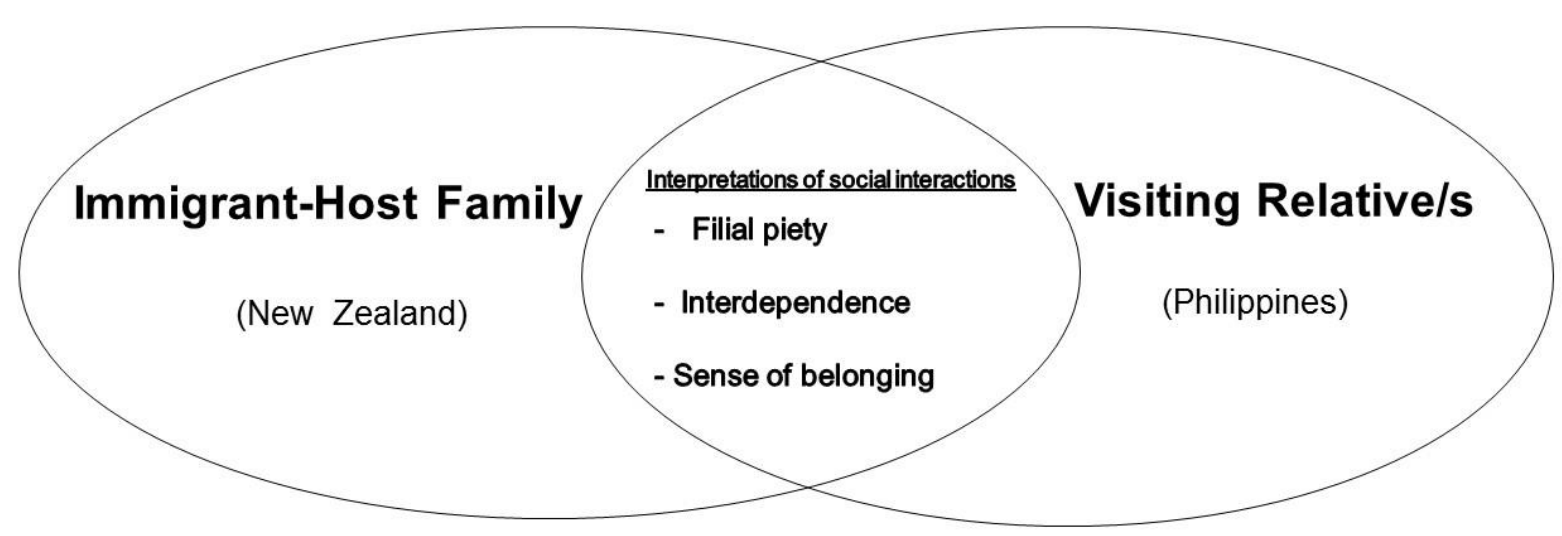

In addition, interdependence as manifested in reciprocally looking after each other's wellbeing and is performed both by the hosts and guests when fulfilling familial obligations. As filial piety is expressed by immigrant-host parents, their families (or family of procreation) can expect that there is a mutual support being provided to VRs that is being fulfilled in New Zealand by their hosts. This type of reciprocity may not be shared from a Western perspective as shown by Uttal (1999) where working class Anglo-American women find it inappropriate to call upon their relatives for child care needs. However, this study shows that the support being provided by VRs (usually the grandparents) which becomes a normative process in terms of them providing care for their grandchildren. There is also a "chain of support" where the assistance being provided by the immigrant-host families is extended to their respective VRs such as the host(s) supporting the wellbeing of their guest(s) in an act of reciprocity. Moreover, this chain of support is not limited just to the VRs (i.e., grandparents) but often extends 
further to the other members of family in the Philippines (usually the immigrant-host's family of procreation and can go farther to extended family members). However, the precise nature of the reciprocal chain of material and financial support was underexplored during the interview process with the immigrant-host adult children.

Upon examining interdependence within a cultural context, the individualismcollectivism conceptualisation as polar opposites may coexist in groups and individuals at the same time in different situations and with different target groups. A study by Kagitcibasi $(2005$, p. 410) regarding autonomy and relatedness and its implication for self and family provided three prototypical family interaction patterns: (a) the traditional family, characterized by interdependence between generations in both material and emotional realms; (b) the individualistic model, based on independence; and (c) a dialectical synthesis of these two, involving material independence but psychological interdependence between generations.

While it is beyond the intended scope of this thesis to categorise these types of interactional patterns, the parenting orientation between Filipino parents/ grandparents or VRs with the immigrant-host parents exhibited interdependence that may either be both material and emotional as reflected in the on-going social exchanges and emotional solidarity between them. This is also apparent between an immigrant-host mothers and their sibling(s) as they still recognise their bond despite each having their own family of procreation. In comparison, the psychological interdependence of immigrant-host children needs further investigation given the diversity of research participants (i.e., those who migrated at a very young age versus those who migrated recently). Although adult children as first-generation immigrants identify the close-knit ties they have with their family of orientation in New Zealand, they are still in solidarity with their relatives in the Philippines (the extended family) and with their VRs in relation to their hosting experience.

Interdependence also has a gendered dimension as most immigrant-host mothers/ wives maintain ties with their family of orientation to gain assistance from them to provide support to their families in New Zealand in times of need (e.g., during post- 
natal recovery or to provide child care while traveling) or to assist them in maintaining their domestic (reproductive) roles within the household while they may have to work outside of the home (the productive sphere), which has become the norm in their new homeland. Nonetheless, the matrilineal pattern observed between the relationships of the host families with their VRs shows that while the latter are taking overseas trips to New Zealand, it is the immigrant-host mothers who act as the main catalyst in maintaining ties between families in the Philippines and New Zealand.

The third and most critical dimension for interpreting the social interactions between immigrant-hosts and VRs is the sense of belonging. While immigrant-host families may have a sense of belonging or attachment with their new homeland, there is also a relational concept that identifies them with their families in the Philippines, which is at the heart of their sense of being "one of us." This dimension becomes apparent when immigrant-hosts and VRs still recognise their traditional familial bonds and are still in solidarity with one another. This was also demonstrated in other scholarly literature in relation to the "return visit" undertaken by diasporic communities (e.g., Duval, 2003; Huong \& King, 2002; Obsequio-Go \& Duval, 2003; Scheyvens, 2007) which reflects how immigrants periodically return to their former homeland to reaffirm their familial ties and they become a "guest." However, what makes this thesis unique from the earlier studies is the context: the immigrant-host families examined assume the role of being a "host" in their new homeland. Specifically, immigrant-host children who did not grow up living in the Philippines express a discernable sense of belonging with their VRs by way of their sharing themselves despite the fact that some of the host-children may not know their VRs very well as they were separated by distance at an early age. Because of these familial ties, the visit may foster a sense of belonging as immigranthost children may be willing to 'host' and get to know their relatives who are from the previous homeland of their parents. This expression of belongingness within the family is then reciprocated by their VRs as "being one with the other."

Earlier, in section 5.4.1 (Hospitality and sociability between immigrant-hosts and VRs), the child-parent relationship between the immigrant-host mothers/wives and their parents are reaffirmed as most daughters perceived the interactions during the visit as 
being "themselves" rather than as being hosts. On the other hand, the majority of the VRs see themselves as family members (as parents or grandparents) and not as guests. Thus, VR travel enhances and furthers the social identities of the immigrant-hosts and their VR guests as those of being a member of a traditional family, as they are able to re-establish the ties that existed in the Philippines through their hosting in New Zealand. VR travel is therefore very critical in teaching traditions and values to the immigrant-host children (regardless of whether they host for VRs or, are making future trips to the Philippines where the immigrant-host children probably would assume the identity of being a "guest" due to the influences of their current homeland on their development). In essence, the social interactions between immigrant-hosts and VRs are captured by Robinson and Lynch in the context of domestic hospitality (2006, p. 177):

Within the domestic discourse, home plays a central role in intergenerational socialisation influencing, conditioning, and shaping inherited societal norms, values, beliefs, ideologies, and contemporary interpretations of the "laws" of hospitality. It informs and defines future generations of hosts/guests, and their expectations and behaviours, including the gendering of relationship and roles.

Generally, the social interactions between immigrant-hosts and VFRs represent an expression of one's authentic self, either as a family member or as a friend. Authenticity lies in connections, not in separation and distance (Hall, 2007). It is further redefined in terms of values rather than as an "object" as interactions become a fluid space where values are generated and performed (see Taylor, 2001). Drawing on Wang's (1999) argument, these social interactions are not merely searching for authenticity of the Other. Rather, these are also searching for the authenticity of, and between, themselves. Performance through hosting and visiting in New Zealand then becomes a means or medium by which they are brought together to express themselves and experience their authentic inter-personal relationship. This expression of authenticity between hosting and visiting friends and relatives as an exchange entails reciprocity where affections more than objects are circulating as it focuses on the good of the person rather than the material goods (Russo, 2014). 


\subsection{Conclusion}

Social interactions between immigrant-hosts and VFRs are complex and dynamic. This involves hosting and visiting families and friends which results in a visiting experience that is best described as a multi-faceted phenomenon. Utilising a hermeneutic phenomenology paradigm provides insights into how friends and family members construct meanings and understandings of their social interactions in the context of VFR travel. Interpreting the social interactions between the immigrant-hosts and VFRs through two conceptual lenses, social exchange theory and theory of emotional solidarity, provides a holistic approach for examining the cultural influences of the social interactions between first-generation migrants and their respective friends or relatives.

In previous scholarly studies there was an element of "othering" when examining hostguest interactions which may not be appropriate when examining experiences in the context of VFR travel as the hosts and guests by definition know each other. Also, there is a lack of research that exposes the details and multiple perspectives of the hosts and guests and reveals insightful differences in relation to the interpretations of the visit from various perspectives. Existing studies of host-guest interactions within VFR travel research are usually one-dimensional, that either focuses on the host or the guest. This study contributed to academic research by providing a better understanding of the similarities and differences in relation to the interpretations of the social interactions between immigrant-hosts and their visiting friends or relatives. Using different theories, the social exchanges between friends and relatives are illuminated to detail their social interactions which are based on trust, reciprocity, and commitment, while emotional solidarity is fostered by sharing and giving of oneself.

VFR travel is primarily concerned with maintaining social relationships between the actors and where the interpretations and understandings of their relationships are derived through the experiences of their social interactions. Interviewing families also provided a gendered dimension of hosting as there are differing expectations to hosting families and friends. Immigrant-host families and VRs or VFs bring different purposes in relation to the visit. The social interactions between immigrant-host 
families and their respective VRs are tied to familial obligations and expressing intergenerational ties through the visit of a member of a host-parent's family of orientation which will expose the children in the family of procreation to a culture and values that they are maintaining across time and space. There is a matrilineal pattern for the social interactions between the immigrant-hosts and VRs as immigrant-host mothers/wives become the catalyst of VR travel in the goal of uniting their family of orientation in the Philippines with their family of procreation in New Zealand. On the other hand, the immigrant-host families and VFs social interactions are exchanges that are based on past interactions by which these actors reaffirm their social identity and their commitment to remain as friends through VF travel. However, the analysis of the temporal dimensions of the immigrant-host's $\left(s^{\prime}\right)$ and $V F^{\prime} s\left(s^{\prime}\right)$ social interactions shows that communications between them invariably wax and wane both before and after the during-visit.

As this study specifically focuses on the first-generation Filipino immigrants in New Zealand and their respective VFs or VRs from the Philippines, the results should therefore only be limited to this immigrant community along with their visiting friends and relatives. Nonetheless, future research should be considered of the hosting experiences of other immigrant communities that examines the multiple meanings of their social interactions with their respective VFs or VRs. Longitudinal studies that examine succeeding generations of immigrants in relation to their hosting may provide a different perspective in terms of understanding immigrant-host families. Since this study focuses only on first-generation Filipino immigrants and their respective VRs, the interpretations of their social interactions are found to be based upon filial piety, interdependence, and sense of belonging. However, the social interactions between first-generation Filipino immigrants and their respective VFs are founded on their shared friendship which is based on their past interactions and nostalgia for their shared history which may have produced positive experiences which both parties wish to continue. In the future, it would be worthwhile to examine whether the interpretations of friendship and family ties provided in this study are eventually "diluted" or perhaps evolve into a different understanding of social interactions critical in sustaining VFR travel across distances, time, and generations. 


\section{CHAPTER 7 - Conclusions and recommendations}

\subsection{Introduction}

This is the concluding chapter of this thesis which has explored how social interactions between immigrant-hosts and VFRs are understood and interpreted by them. This thesis has examined the host-guest interactions of first-generation Filipino immigrants in New Zealand and their respective VFs or VRs from their former homeland, the Philippines. This chapter begins by revisiting the research questions in section 7.2 before considering the theoretical and methodological contributions of this study outlined in sections 7.3 and 7.4, respectively. The limitations of the study (section 7.5) as well as the implications of the methodology and theory to the tourism industry and policy are also discussed (section 7.6). Opportunities for future research related to VFR travel (section 7.7) are also noted before the conclusion to this final chapter (section 7.7).

The contributions of VFR travel are underestimated in the tourism industry and as an academic discourse. A critical element of this study involves giving voice to immigrant families and their respective VFRs as both are often under-acknowledged and/or under-represented within tourism research, specifically when examining social interactions as the former have settled in rather large numbers in their new homeland, New Zealand, and where they in turn receive their respective guests. Specifically, members of the hosting family (parents and their adult children) were interviewed in relation to the interpretations of their hosting experience in their current homeland, to document the complexities involved when interacting with their guests, whether they are VFs or VRs. This research has used a rich and distinctive method of examining social interactions of all of the respective actors, which would include the friends and family members, for both the hosting and visiting parties. In essence, this study has interpreted the social interactions of non-Western research participants (being firstgeneration Filipino immigrants and their respective Filipino guests from the Philippines) that take place in a multicultural setting of New Zealand, using social exchange theory and the theory of emotional solidarity. 


\subsection{Research questions revisited}

To be consistent in terms of the presentation of the key findings of the study, this section begins by answering the four supplementary questions before addressing the main research question as provided below:

\section{Supplementary question \# 1: "What is the nature of the social interactions between} immigrant-hosts and their VFRs?"

It was revealed that the nature of the social interactions of VFs and VRs with their hosts was significantly different. VFs are frequently found to be itinerant and less dependent on their hosts while the VRs are mostly interdependent with their host families and were therefore less mobile. The trips undertaken by VFs are frequently a product of complex interactions between the different host families being visited during their travel. The trip being undertaken by VFs are often carried out as a series of visits with the aim to reconnect them with networks (either friends or relatives) as VFs visiting New Zealand usually have multiple motivations for travelling to New Zealand. When hosting friends, immigrant-host families may collaborate either within the household or contact other relatives and friends to find accommodations for their VFs at other locations. While VFs were identified as being itinerant guests, VRs were anchored to and stayed with their hosts during their entire visit and for significantly longer periods of time although, some VRs did travel around or go out with their hosts. Nonetheless, they were categorised as interdependent guests in reference to their social interactions with the immigrant-host families which were very intertwined with mutual familial obligations.

The hosting of friends leans toward expressing hospitality as a way of sharing oneself and guests derive pleasure from the visit despite the fact that VFs pay shorter visits than VRs pay with their host family. The visit of VFs is more inclined towards the mutual enjoyment of touristic venues and VF trips are less structured by obligation and have a greater voluntaristic element. On the other hand, hosting relatives almost invariably entails VRs to fulfil an obligation or provide support for an indefinite or long period if 
necessary. As the relationship between immigrant-hosts and VRs is interdependent, the nature of social interactions between them is a reciprocal exchange where family members can expect support from one another. Thus, there is a propensity for anticipated return visits for VRs which is not reflected in the social interactions between immigrant-hosts and VFs. Also, the exchanges between friends occur such that one may make contact when they plan on visiting but communication between them can wax and wane as there is no need to consistently and regularly communicate as their lives are not as multidimensional and intertwined as those of family members. However, what is similar is that in both cases social interactions are complex and dynamic involving the various members of the hosting family in varying degrees, together with their respective guests (VFs or VRs) according to the dynamics of their relationships.

\section{Supplementary question \# 2: "What are the social exchanges between the immigrant-hosts and their VFRs?"}

It emerged that hosting and visiting as a social exchange means that there are on-going relationships that the participants want to maintain even across great distances. While the nature of the social exchanges between families and friends are separate and distinct, these social interactions are generally based on trust and within the principles of reciprocal exchange. However, the social exchanges between immigrant-hosts and VFs are rooted in their past interactions, and particularly in the Philippines where they had established and strengthened their ties until the hosts eventually migrated to their new homeland. The unwritten obligations of friendship are focused on expectations of aid and/or solicitous behaviour growing out of assumed bonds of investment, commitment, and reward dependability which fulfil a friend's need. This was apparent among the immigrant-hosts who are expected to carry out their duties as hosts for their friends. The mutual liking, shared experiences and trust are essential elements of friendship by which social interactions further enhance these qualities through the during-visit phase between friends. Social interactions allow them to re-establish their social exchanges after being separated by time and distance. It also allows the compressing of time and space where friends re-acquaint themselves with one another 
during a finite period of time over the course of a visit in order to renew their friendship ties and create new memories.

On the other hand, the social exchanges between relatives are based on interdependence and this assumes that there is a trusting environment within the family or among relatives as there is mutual commitment to help one another. The social exchanges occurring during the hosting or visiting of relatives are intertwined with interdependence, caring for others, and familial obligations. The obligation to travel on the part of the VRs and for hosting by immigrant-host families creates dynamic and reciprocal relationships through the fulfilment of duties within the family. Such social exchanges are focused on providing support, nourishing and maintaining relationships between family members. There can also be a chain of exchanges and reciprocity occurring between VRs and the immigrant-hosts that is further extended as the former assist the immigrant-host family while the latter become committed to help their relatives in the Philippines in a time of need.

\section{Supplementary question \# 3: "How do social interactions reflect emotional solidarity} between the immigrant-hosts and VFRs as expressed by their shared beliefs and behaviours?"

The emotional solidarity manifested between and among friends and families through VFR travel is rooted in the sharing and giving of oneself. The emotions that individuals experience as a result of social interactions, either through the hosting and visiting of friends and relatives are likely to be perceived as jointly produced. For friends, the affective nature of social interactions includes the sharing of personal thoughts and feelings that are expressed through the hosting and visiting of friends. Hosting and visiting in the context of VF travel is a way of acting and being with friends in real time which affords the opportunity for shared experiences and facilitates maintenance and advancement of the relationship.

Despite limited contact in the pre-and post-visit phases of the social interactions, the co-presence of friends through the visit enables sustaining relationships that are not 
based on proximity, but are continued over periods of time between the particular actors. Indeed, togetherness plays an essential element in determining the quality of the social interactions between immigrant-hosts and VFs. The temporal dimensions of social interactions reveals that there is an intense period of social interactions that is preceded by and followed by sparse or infrequent contact. This has been an observed pattern where the nature of friendships is separated by distance. Hence, the social interactions between friends are fostered through "connectedness in togetherness."

Meanwhile, the emotional solidarity shared between the immigrant-hosts and VRs is expressed through the shared identities in relation to their significant others where both actors feel "being at one" or "in union" with the other. Whether it may be reinforced by communality, places, events, or situations, it is the co-presence of the significant other that produces a positive emotional experience, giving the immigranthosts and their VRs the sense of solidarity. In the same vein, hospitality and sociability between immigrant-hosts and VRs are illustrated by the shared identities that they express in being family members. They are also an expression of how much one is being valued as a family member by making social interactions "extraordinary" during the everyday life (on-site interactions or during-visit phase) of the immigrant-hosts and VRs. Even if the social interactions of the immigrant-hosts and VRs were examined across the temporal and spatial dimensions, they still cite "co-presence" as being remarkable and a factor which cements their relationships.

\section{Supplementary question \# 4: "How does culture shape social interactions between the immigrant-hosts and VFRs?"}

The data revealed that culture shapes social interactions differently between visiting friends and family and their hosts. However, commonality also exists which is a product of intercultural exchange between these actors. Friendship as a universal experience incites and enables the social interactions between immigrant-hosts and VFs. The act of hospitality being expressed is manifested by the friendship being shared by people within a social interaction. A fusion of non-Western and Western perspectives guides the social interactions when the immigrants host friends offer hospitality that is 
influenced by the culture of their former homeland as a form of interpersonal relationship with their VFs even when it is expressed in a different cultural setting. The perceptions and experiences of the hosts and VFs reflect their respective notions of hospitality in terms of cultural norms and expectations of hosting and visiting. In addition, friendship is shared by immigrant-hosts and VFs and is linked to the social exchanges and emotional solidarity that results in the mutual trust and commitment and the affective dimensions of sharing and giving of oneself between friends. Friendship during visiting and hosting is performed and continued through each other's co-presence in the setting of the immigrant-host's $\left(s^{\prime}\right)$ new homeland. A gendered dimension of hosting for friends also reveals an interesting insight with regard to domesticity and familiarity, as the domestic aspects of hosting becomes mainly a part of the duties of immigrant-host mothers/wives, even if she is a secondary-host to her domestic partner's VF.

Similarly, typical VR travel occurs due to gendered and cultural expectations within the two types of families - the family of orientation in the Philippines and the family of procreation in New Zealand. As VR travel reflects a matrilineal pattern, the interdependence between immigrant-host mothers/wives with their relatives in the Philippines occurs because of their efforts to incorporate their family of procreation (in New Zealand) with their family of orientation (in the Philippines). Likewise, the transplanting of the motherhood roles of Filipino women in New Zealand elicits interdependence as immigrant-host wives/mothers may need assistance from their family of orientation in maintaining their domestic roles within their family of procreation in their new homeland. For the parents within the immigrant-host families, the ongoing exchanges between them and their VRs may also be influenced by filial piety. However, there are cultural differences between the social interactions of the immigrant-host children with their respective VRs which may elicit conflict or misunderstanding as they may no longer share the same traditional Filipino cultural values that their guests may possess.

The four supplementary research questions that inquire on the various aspects that influence the social interactions between immigrant-hosts and VFRs including the 
nature of their social interactions, social exchanges, emotional solidarity, and culture are brought together to answer the main research question: "How are social interactions between immigrant-hosts and their visiting friends and relatives understood and interpreted by them?"

Hosting and visiting families and friends become an authentic expression and sharing of themselves by "being together" within a shared life experience. The common meaning and understanding lies in searching for the authenticity of, and between, them. In the context of Filipino relationships, it also means "being one with the other" or pakikipagkapwa. Although the social interactions between immigrant-host-VR and immigrant-host-VF are different, as the latter pair reflects a purely and mutually voluntary relationship as compared with kinship or familial ties which are nonvoluntary, both sets of social interactions are understood as maintaining their interpersonal bonds. VFR travel provides a fluid space for inter-cultural engagement, encounters, and exchanges. It emerged that the social interactions between immigrant-hosts and VFRs represent an expression of one's authentic self - either as a family member or as a friend. Examination of these social interactions reveals that they are multi-faceted and layered, which generates a range of themes encompassing time, persons involved, places, and culture. The co-presence of relatives and friends requires that they have the opportunity to meet and spend time together as occasional physical proximity enhances and sustains relationships.

Each social interaction has a different meaning for individual participants and the meanings vary depending on each participant's perceived value of the relationship that was established between a specific immigrant-host family member and their respective guest/s. The secondary actors are sympathetic to the primary actors and are supportive of their interaction. For instance, the social interactions between immigrant-hosts and VFs provided different meanings and understandings that elicit social exchanges through an on-going mutual trust and commitment as they continue showing concern, sympathy, and openness in a different setting. Emotional solidarity enhances friendships through one's co-presence, re-acquaintance, and re-establishing friendship ties as hosting and visiting provides an opportunity for friends to spend time together 
since periodic or even sporadic physical proximity is critical in sustaining friendships across time and distance. These social interactions are mutually accorded as individuals can still recognise and are drawn to reaffirm the friendship with their significant others despite the fact that the immigrant-hosts are living overseas in New Zealand and are immersed in a different cultural setting that is far from their friends who still reside in the Philippines. In the context of primary actors in the social interactions between immigrant-hosts and VFs, it emerged that the hospitality being shown by immigranthosts to their VFs is a result of their friendships being shaped by continuing social exchanges and emotional solidarity through their past interactions.

Meanwhile, secondary host(s) (the other host-family members) and other ancillary member(s) of the principal guest's party may still foster solidarity during the visit. While secondary hosts and secondary guests provide and receive hospitality, respectively, through their association with the friendships of the primary hosts and guests, the meanings of the social interactions for them are influenced by the cultural norms of hosting for guests, while the VFs are guided by their cultural expectations of hospitality when being hosted.

Comparatively, social interactions also influence the depth of the shared experiences of the immigrant-hosts and VRs by re-establishing and renewing family bonds through family reunification and the fulfilling of obligations within the family. There are three underlying dimensions in interpreting the social interactions between immigrant-hosts and VRs. These are: (1) filial piety; (2) interdependence; and (3) sense of belonging. The social obligations to host by immigrant-relatives and to travel on the part of the VRs create dynamic and reciprocal relationships through the fulfilling of duties among the family. Most social interactions between immigrant-hosts and VRs follows a matrilineal pattern where the meanings of the social interactions for immigrant-host mothers/wives cuts across the three dimensions for interpreting the social interactions which were noted earlier. This pattern catalyses VR travel which promotes continuing intergenerational ties that connects relatives in the Philippines with their family in New Zealand. 
In contrast, the social interactions of the immigrant-host fathers/husbands are articulated by mutual exchange or reciprocity with their VRs/in-laws and are expressed by demonstrating that they have the adequate material resources to properly host and that they have accomplished their role of advancing their family's fortunes by immigrating to their new homeland. Immigrant-host children provide varying interpretations of their social interactions depending on their affinity with their VRs, but by and large they related sentiments that encompassed re-acquaintance and reciprocating the relative's(s') visit through their co-presence and time spent with their VR. In comparison, the interpretation of social interactions for visiting relatives as 'guests' is oriented towards the wellbeing of their host(s) which is an expressions of gratitude, altruism and compassion.

\subsection{Theoretical contributions}

This study developed a conceptual understanding of the social interactions between immigrant-hosts and VFRs at the micro-level. The depth and breadth of interpreting the different meanings of social interactions between hosts and guests involved in VF and VR travel is a notable contribution of this thesis. Specifically, this thesis contributes to the academic discourse encompassing tourism and migration, host-guest interactions, and VFR travel as:

- Most of the previous research that analyses the relationship between tourism and immigration addresses macro-level flows of permanent immigrants and VFR travellers but, has not addressed the micro-level interactions between and among the actors. In the same vein, the relationship between tourism and migration has consistently been analysed based on the paradigms of production and consumption. This research has examined the micro-level interactions between hosts and guests by exploring the different meanings of their interactions;

- There is an element of "othering" that was discovered when examining host-guest interactions, which is different in the context of VFR travel. This study recognises that similarities do exist between the host and guest as they know each other through friendship or kinship; 
- Existing studies of host-guest interactions within VFR travel research are usually one-dimensional; usually they either focus on the host or the guest. There is a lack of research that provides the multiple perspectives of the hosts and guests and reveals insightful differences in relation to the meanings of the visit from various actors involved in VFR travel. A holistic approach of this study is that it addresses this gap by not only giving a voice to the hosts, but to their families including adult children as well as their respective VFs or VRs; and

- The interpretations of friendship in the context of VF travel research are absent within tourism scholarship. In addition, this research has elicited the multiple meanings of "the visit" among family members that includes parents and adult children together with their respective VRs. Hence, this study has examined the social interactions between immigrant-hosts and VFs and immigrant-hosts and VRs as two distinct phenomena.

This study has sought to contribute to filling these gaps in tourism research and reveals that the social interactions between immigrant-hosts and VFRs are dynamic, multidimensional and multi-faceted when examined from the multiple perspectives of the hosts and guests. While tourism research reflects "the tourist" as a metaphor of the social world (e.g., Dann, 2002; Uriely, 2005), this study brings together at the core of the analysis, both hosts and guests, who are interacting in the context of VFR travel.

This thesis has examined host-guest interactions at the micro-level through the conceptual lenses of social exchange theory and the theory of emotional solidarity. The absence of an established framework for understanding the meanings of social interactions between hosts and guests in the context of VFR travel led to examining the social phenomenon through these theories. This study has provided a holistic approach for examining the multiple perspectives involved in the host-guest interactions and also in the conceptualisation of VFR travel using different sociological theories and how they are used in tourism studies as these theories have not been utilised to examine the social interactions of hosts and guests. The interactions lie at the core and drive VFR travel and this approach considers the previous studies which have focused on the 
exchanges and solidarity between the "self" and "others." Further, the utilisation of these theories has provided a richer approach of examining host-guest interactions that is also multi dimensional (pre-, during-, and post-visit) and taking place in a noncommercial setting. It also contributes to the way that both of these theories are applied and as a result offer deeper insights on the social exchanges and emotional solidarity through a cultural context using Filipino indigenous philosophy within a multicultural setting.

Approaching the phenomenon through hermeneutic phenomenology, this research was able to develop other theoretical insights to further interpret the experience and inform concepts that arise from interpretive analysis (Pernecky \& Jamal, 2010). For instance, reframing the conceptual lenses utilised in this study indicates that the social exchange and emotional solidarity between immigrant-hosts and VFRs reflect on-going trust, commitment and reciprocity by sharing oneself. Interpretation of the meanings of the social interactions between immigrant-hosts and VFs from those of the immigrant-hosts and VRs reveals that friendships are voluntary and less complex than the multidimensional and interdependent nature of family ties.

This study has also contributed to examining the cultural influences on social interactions between a specific immigrant-host community in relation to their VFRs which may be valuable in furthering overall understanding of VFR travel. In particular, this research has examined VFR travel by merging both Western and non-Western perspectives in analysing the social interactions of first-generation Filipino immigranthosts in New Zealand and their respective VFRs from the Philippines that is taking place in a multicultural setting. This research was able to gather and construct the multiple meanings of the social interactions between immigrant-hosts and VFRs and how both Western and non-Western cultures shape their social interactions. Despite borrowing traditional Western epistemologies in interpreting the social interactions between hosts and guests, this approach was fused with the Filipino philosophical and psychological studies. However, this study is not an exhaustive overview of Filipino culture but provides an understanding of what is most relevant to understanding the social interactions between immigrant-hosts and their VFRs. Nevertheless, as an 
international tourism scholar trained in an Anglo-American setting, this study has broken new ground and reconceptualised new theoretical approaches to the study of host-guest interactions. After highlighting the theoretical contributions of this study, the next section will focus on the methodological contributions.

\subsection{Methodological contributions}

This thesis addresses the complexity of studying social interactions between hosts and guests in the context of VFR travel, both methodologically and ethically (see also Capistrano, 2013). Specifically, the study captures the nature of social interactions occurring through VFR travel that encompasses multiple perspectives, multiple dimensions, and multiple sites. This study recognises that VFR travel as a phenomenon is multi-faceted, complex, and dynamic involving families and friends who are interacting across time and distance. A recent conference communication by Palovic, Kam, Janta, Cohen, and Williams (2014) further suggests the need for new types of research methods in VFR travel such as examining how the "closeness" of relationships may shift over time due to distance. The application of methodological and ethical approaches in studying VFR travel in the context of host-guest social interactions is underdeveloped, which this study was able to address.

Positioning this study through hermeneutic phenomenology enables studying the common meanings and differences in interpreting the social interactions between families and friendships, which may be difficult to address using a positivist approach. Eliciting multiple perspectives involved interviewing both hosts and guests in relation to understandings of their social interactions. This involved obtaining the different perspectives of the following: (1) the immigrant-hosts of VRs; (2) the VRs; (3) the immigrant-hosts of VFs; and the (4) VFs. In particular, individual interviews were conducted in order to allow host family members and their respective $\operatorname{VF}(\mathrm{s}) / \mathrm{VR}(\mathrm{s})$ to provide the individual meanings of their social interactions that are unique to each of them. This was supplemented by group interviews of the host families which allowed the perspectives of multiple family/group members which further reflected their 
disagreement and/or consensus in relation to their social interactions regarding their visiting significant others.

While other tourism research has elicited the multiple voices by capturing a wholefamily perspective that involves both the parents and children (e.g., N. Carr, 2011; Schänzel, 2010a, 2010b; Schänzel et al., 2012), these studies involve families in the context of generic holiday and travel experiences. However, this thesis gathers the perspectives of a small sample of immigrant families in New Zealand in relation to their hosting experience within the context of VFR travel. However, a specific limitation of the study is that of being unable to interview young children due to potential ethical reasons (see Eder \& Fingerson, 2001). Future studies may involve interviewing young children in order to explore the meanings of their social interactions. This may involve different methodological and ethical procedures.

This study is also multidimensional as immigrant-host families and their respective VFs or VRs were examined at the post-visit phase of their social interactions. While postvisit interviews entail the recollection of experiences for both the hosts and guests, this becomes a holistic approach helping the understanding of the dynamics and processes that are occurring within the social interactions between the participants. Interviewing both hosts and guests in the during-visit phase may also be possible which may be less resource-intensive in terms of the researcher traveling to multiple overseas locations but would require precise timing on the part of the researcher in order to conduct the interviews after the visit and just prior to departure of the visitors. However, progression in the social interactions between hosts and guests from one phase to the next is underexplored and that these study has given a structure to understand such complexity by inquiring on the three stages of time (pre-, during-, and post-visit). Methodologies could be designed for interviewing using virtual means (i.e., Skype) and may aid in reducing the travel logistics and costs of studies of this type. However, the interview would lack significant details and nuances as compared to interviews conducted face to face. 
As the study is multidimensional, it follows that interviewing the hosts and guests separately is also multi-sited as first-generation Filipino immigrant families and the VFRs were interviewed in separate countries of New Zealand and the Philippines, respectively. As Pearce (2014) described the internationalisation of tourism research as a process whereby components and methods of the research are international in nature, this study developed a more integrated approach for analysing and interpreting VFR travel as a phenomenon across international boundaries. Additionally, interviews in New Zealand were also multi-sited not only because of the difficulty in recruiting families within one city (Wellington), but also due to compensating for the inherent intimacy among Filipino immigrant community in the study area as specific or unique anecdotes risk giving clues to the identities of the participants to other community members. Hence, recruitment was also undertaken in Auckland to achieve the targeted number of families hosting friends and relatives and to aid in preserving the privacy of the participants.

\subsection{Limitations of the study}

Apart from the methodological limitations presented in section 3.6, the main limitation of this study is that the social interactions between immigrant-hosts and VFRs as a phenomenon are not generalisable due to the limited number of participants and, therefore, lack of representativeness. In particular, the social interactions between hosts and guests only reflect aspects of Filipino culture and that different findings may emerge should an analysis be untaken of other immigrant-host communities in New Zealand and their respective VFRs. However, when examining social interactions in the context of Filipino culture, emotional transnationalism is also mirrored particularly in the responses of immigrant-host children (section 6.6) where privacy remains important for them while also questioning some Filipino norms and values that their parents have in relation to hosting for their guests.

The study also lacks representativeness due to the complexity of relationships that may occur between the immigrant-hosts and VFRs. A whole-family perspective was difficult 
to achieve in eliciting the views of immigrant-hosts that includes at least one parent and one adult child. The voices of younger family members, particularly the children under the age of 18 years old, were not included due to potential ethical reasons. The desire of the researcher to not create any semblance of impropriety, and his lack of experience in interviewing minor children were explained in section 3.6. Future studies may consider including young children and analysing their social interactions in the context of VFR travel while taking into account any ethical implications. Another issue that should be addressed is whether young children were actually able to act as host or perceive themselves as such. It is suggested that a different approach should be considered in terms of interactions of children with their respective VFs or VRs. Interviewing children within the hosting family could be undertaken by other scholars and could address the question: To what extent are young children actually able to act as hosts or even consider themselves to be hosts for VFs or VRs? After consideration of this question then, different approaches may be further explored to interpret the meanings of the social interactions of the young children with their respective VFs or VRs who visit them.

As previous tourism studies have focused on family research, the context under study usually deals with holiday experiences. However, there are various complexities involved within the social interactions underpinning VFR travel. Unlike adult children who were straightforward and realistic about conflict with their VRs or the challenges confronted in hosting (section 5.6), the researcher believed it may be indelicate or risky to inquire about personal matters and pose questions in the area of human relations to young children as they may not be aware of discretion even if they are being supervised by their parents. Interviewing young children may need a different set of questions that would avoid focusing on the negative aspects of hosting or having a guest so as not to create a conflict or embarrassment between their parents who may be listening to them during an interview (e.g., a child not liking the visit of a maternal or paternal grandparent or any other relative for that matter that may offend either one of his/her parents). In contrast, adult children are able to discretely articulate and defend their ideas particularly in a group interview especially when they disagree with their parents. 
There is also scope for reflection as to whether a whole-family perspective may be necessary when interviewing immigrant families who have hosted for VFs. For instance, there may be a case when only one member of the immigrant-host family has a significant bond with the VF(s). However, this is not the case when examining the social interactions between immigrant-hosts and VRs as their ties are rooted through kinship and those relationships are continuously being maintained across distances. This study is limited to a specific interaction between one immigrant family and their respective VFs or VRs. Also, the complexity of visits by VFRs (e.g., contemporaneous visits by VFs and VRs) on the dynamics of social interactions were not explored and it does not attempt to account for the effects of a simultaneous visit of VFs and VRs to a particular immigrant-host family.

There is scope to extend family-based research in tourism studies by examining a range of familial relationships within the context of either domestic or international trips. Relationships between siblings could be explored as well as ties between, for example, cousins. Although there were two VRs in this study who were sisters of an immigrant-host mother, there are certainly opportunities for others to explore sibling relationships in more depth - and ties within the extended family - that are in some way linked to host-guest interactions. A family consists of a complex array of relationships, and this study could not possibly capture all of these. Other forms of ties that recognise the complexities of families may be investigated in the future to examine the immigrant-hosts and VRs as a social phenomenon (see section 7.7). Also, the social interactions between immigrant-hosts and VFs are also limited to friendships which immigrant-host families and VFs had initiated in the Philippines. The study was not able to recruit or examine other forms of friendship such as those involving romantic relationships (e.g., adult immigrant-host child maintaining a relationship in the Philippines) that would involve different social interactions between hosts and guests.

The post-visit interviews relied heavily on the memory and recall of the immigranthosts and VFRs as this study deals with only the most recent visit. Memories can be 
unreliable at times, and photo-elicitation was particularly useful in this regard as it gives respondents more opportunity to reflect before responding. It also proved useful when dealing with older/aged respondents who may need additional support when it comes to recalling past events. Unlike when interviewing VRs, the potential of photoelicitation was not maximised when dealing with immigrant-hosts (both for hosting families or friends) and VFs due to time constraints and the lack of availability of private space to conduct in-depth interviews.

There is also difficulty differentiating and interpreting the responses of the participants for the individual and group interviews. While there are some differences in the responses of the participants (e.g., motivations, expectations), there are several instances when the responses provided by the individual and group interview questions are the same which presumes that decisions for hosting and visiting friends or relatives is a communal decision being undertaken by different parties. Therefore, hosting and visiting friends and families could arrive at a consensus on the agenda and scope before a trip is undertaken. Nonetheless, this researcher reflects upon the duration of group interviews that were usually carried out during late evenings (after all the entire individual interviews were done) and they resulted in providing virtually the same responses for both individual and group interviews. However, the obligatory nature of VR travel itself may elicit similar responses between family members both in the Philippines and New Zealand. The consensus found in hosting and visiting for relatives as a phenomenon was not apparent with hosting and visiting for friends due to the limited number of research participants. Future studies may explore the range and complexity of group dynamics that occur when hosting and visiting for friends.

Moreover, the negative dimensions of VFR travel such as the difficulties associated with hosting or visiting did not emerge as a prominent theme compared to the study conducted by Schänzel et al. (2014) who examined the hosting experiences of Polynesians in New Zealand. There are several assumptions that may explain why any untoward incident of hosting or visiting families and friends did not surface in the interviews: 
- First, there is an ongoing mutual interdependence between immigrant families and VRs (section 5.3.1) that entails helping one another in times of need. Despite their old age, VRs are making sacrifices to assist the immigrant-host families with child care in exchange for financial support being provided by the hosts not only to the VRs but also to other relatives in the Philippines.

- Second, the expression of filial piety (section 5.5.1) among immigrant-host mothers is still underpinned by their pakikipagkapwa (being one with the other) with their VRs, a dominant Filipino cultural value. Given this situation, they still show their utmost respect to their parents who are visiting them.

- Third, immigrant-host fathers/husbands did not share any negative aspects of hosting for their VRs. Within the domestic sphere, this may be attributed to the benefits derived from having a VR (usually the mother-in-law) assisting the host family in order to unburden their wives from household duties. There are also occasions when the author would ask questions of the immigrant-host fathers related to the previous visit of their parents. While previous hosting experiences are not analysed in the study since this is beyond the scope of the study, comparing the previous visits of the husband's family of orientation versus those of the wife's family of orientation reveal a pattern that relatives from the husband's side tend to have shorter stays. If this scenario is contrasted to the matrilineal pattern found in hosting the relatives within the immigrant-host mothers' family of orientation, it is possible that the obligation of hosting and/or visiting reflects the multiple roles that Filipino women carry within a New Zealand household that immediate relatives are obligated to travel and assist them in time of need. However, future studies may examine the hosting of the husbands' family of orientation as a different phenomenon.

- Fourth, the shorter visit of VFs who were all first-time visitors to New Zealand and whose visit with their friends range from three to ten days may not be of a duration that is long enough to develop significant conflicts. Additionally, there are also norms and expectations within Filipino culture that the hosts should express utmost hospitality to their guests (see section 4.6).

- Last, the audio-recorded interviews may incite families and friends to not provide any negative information in relation to their social interactions. From a cultural 
perspective, Filipinos are taught that all problems should be discreetly kept within the family (Wolf, 2002).

Regardless of any negative aspects within the social interactions of the immigranthosts and VFRs, friendship and kinship are still being valued whether for personal gains or for the common good. This is reflected in how families and friends express the importance of maintaining and deepening the ties through the visit and bolstered through their social exchanges and emotional solidarity. Nevertheless, the researcher still upholds the highest ethical standard in assuring that what is shared or entrusted by the research participants would not have any adverse effect on their relationships with their friends and families. The implications of the study to the tourism industry and on policy development are discussed in the next section.

\subsection{Implications for the tourism industry and policy}

This study is based on the social interactions between first-generation Filipino immigrants in New Zealand and their respective VFRs from the Philippines. As past research focuses on social interactions at the micro-level, the practical implications of this study may be beneficial for the tourism industry and immigration policies and advances understanding of the "new" or "other" residents as they become both producers and consumers in their new homeland. As Yeoman et al. (2012) predicted for the future of New Zealand's tourism, one of the drivers for this industry is the changing population of the nation as it becomes older and demonstrates more ethnic diversity.

Recently, the Tourism Industry Association of New Zealand (TIANZ) has disseminated its strategic framework entitled "Tourism 2025" with a long-term vision of benefitting New Zealand tourism and the wider economy. ${ }^{20}$ In particular, the industry has acknowledged the value of the Chinese market in terms of inbound tourism as well as

\footnotetext{
${ }^{20}$ See summary report on http://www.tourism2025.org.nz/assets/Documents/Tourism-2025Summary.pdf
} 
recognising the potential of emerging economies of Indonesia, India, and Latin America. Whilst TIANZ places much importance on the 90,000 international students that are studying in New Zealand, in terms of synergising VFR travel, the tourism generating potential of immigrants that are being approved annually by Immigration New Zealand (INZ) has not been recognised in the strategic planning framework. However, a current quantitative study by Dwyer, Seetaram, Forsyth, and King (2014) in Australia describes VFR travel as being closely associated with the history and development of international migration patterns. These authors also suggest that tourism stakeholders should consider migration numbers and patterns as these changes will influence various tourism market segments. As TIANZ recognises the value of visitor experience in tourism planning, immigrant communities may also contribute to achieving this goal that may be beneficial not only to the national economy, but also to enhancing the relationship of hosting and visiting families and friends.

Whilst regional and local tourism organisations, policy makers, tourism operators, and investors focus on the economic contribution of VFR travel, understanding the culture of the "new" and "other" residents will enable them to serve the needs of the immigrant communities as well as their respective VFRs. As the trend continues where international migrants tend to live in the bigger cities such as Auckland, Christchurch, Dunedin, and Wellington (Statistics New Zealand, 2014b), this should open better opportunities for other regions (e.g., Queenstown, Rotorua, Taupo) to maintain or enhance the tourism products while reaching out to immigrants in terms of information campaigns about their new homeland which would lead to enhancing their national identity as New Zealanders. However, this is a two-way process where immigrant communities should be able to learn and have a sense of ownership of what New Zealand has to offer and what they can also give to their nation.

Eventually, enhancing local knowledge for the immigrants may lead to a better appreciation of the significant aspects of their new culture and environment that would catalyse both domestic and international travel where immigrants explore their new homeland while being capable of better showcasing their country to their VFRs from overseas. The changing demographics of New Zealand and becoming multicultural also 
means that New Zealand should become more multi-lingual and young New Zealanders should become fluent in a second language (Coventry, 2014b) which would also benefit succeeding generations of immigrants who may no longer be fluent with the tongue of their ancestors.

With regard to civil aviation, leading airlines in the Philippines may examine the benefits of expanding its route to New Zealand to serve the needs of the Filipino immigrants and New Zealanders. Recently, the United States has upgraded the civil aviation status of the Philippines to operate new direct flights (Reuters, 2014), while the European Union has lifted a ban to the Philippine Airlines (the country's flag carrier) and Cebu Pacific (European Commission, 2014). Cebu Pacific is a leading low-cost carrier in the Philippines which has expressed an interest in expanding its services to become the official carrier to New Zealand after an agreement between these two countries (ABS-CBN, 2014; Bradley, 2014). This airline company could utilise its fifth freedom rights which allow the airline's right to fly passengers to a third country from a country with which an airline's resident country has an outstanding air services agreement. In this case, Cebu Pacific can also evaluate its route not only in New Zealand, but also to Australia in order to serve the Filipino immigrants and increase the potential for international tourists (Coventry, 2014a).

In terms of immigration policy, INZ may also need to segregate its data in determining VFs versus VRs. During the recruitment of participants phase for this thesis, an inquiry was made to the institution's office in the Philippines to request data about visiting friends and visiting relatives as separate categories in order to rationalise the difficulties in recruiting hosts for VFs. However, INZ explained that they do not separately categorise the visitor visa applications made by Filipinos as to their relationship(s) with their host(s) such as whether they are a relative or a non-relative (i.e., a friend). Additionally, partnership-based visa applications can also, in some cases, be defined as a "friendship" (E-mail communication with INZ, October 12, 2012). In this case, INZ define "partners" as either those who are currently residing together permanently or having recently done so, and are in a genuine and stable relationship (Immigration New Zealand, 2012). 
Additionally, INZ has a mission to "bring the best people to New Zealand to enhance New Zealand's social and economic outcomes" and may need to examine further its policies that may affect the wellbeing of the immigrants (INZ, 2013). This enabling policy will definitely attract young immigrants (and their families) from other countries (whether developed or developing nations) who have transferrable skills that they can offer to New Zealand. However, as an immigrant-receiving country, immigration policies should also be responsive to the needs of the immigrants. For example, Canada has recently developed a mechanism to grant a special visa to parent or grandparent of a Canadian citizen or a permanent resident called "parent and grandparent super visa" (Citizen and Immigration Canada, 2013). While INZ has a similar mechanism called "parent and grandparent multiple entry visitor visa" ${ }^{21}$, the validity period of the visa is restricted and visiting (grand)parents would therefore need to pay again to renewing their visa which is usually a cost that is shouldered by immigrant-host families. Given that young immigrant families may need family support to aid them in adjusting to their new homeland, they may need their VRs to visit for longer periods to assist with domestic responsibilities or obligations for a longer period of time. However, it is also important that the occupational health and safety of the visitor is protected which the Canadian immigration service requires as VRs should have valid medical insurance coverage for a year and should have passed an immigration medical exam should they need to stay longer than six months. Otherwise, a regular tourist visa would be sufficient.

\subsection{Future research recommendations}

This research provides a foundation for future study of host-guest interactions in the context of VFR travel. It is through a qualitative approach that has elicited the multiple meanings of the social interactions between friends and families and particularly for immigrant communities (first-generation Filipino immigrants to New Zealand) and their respective VFRs from the Philippines. The global demographic change shows the

\footnotetext{
${ }^{21}$ See further information on: http://www.immigration.govt.nz/migrant/general/generalinformation/qanda/multipleentry.htm
} 
importance of immigrants and other ethnic communities as being a substantial resource that acts as a catalyst in promoting VFR travel. Further exploration of the social interactions between these actors should provide a holistic approach for considering the perspectives of both the hosts and guests. Subsequent research may be done to address the future trends in VFR travel research and result in the development of novel theories. This may include:

- Future studies that examine the multiple meanings of friendships and kinship in the context of VFRs of other immigrant or ethnic communities;

- Future research on hosting VFRs by families while recognising that this social structure as families are complex and dynamic and should include single parents, gay/lesbian families, step relations, extended families, and multi-ethnic families while acknowledging any methodological and ethical implications and limitations;

- Future research comparing families who host both for VFs and VRs or studies examining the host-guest interactions in the context of domestic VFR travel;

- From a generational perspective, future research should be carried out on the multiple-meanings of VFR travel from the succeeding generations of immigrants. For example, New Zealand has a very young demographic in terms of the immigrant population of Filipinos. A comparison of the results of this study may be done vis-à-vis second- or third-generation of Filipino immigrants in the United States, particularly in California, Hawaii, and Nevada where the Philippine language is the most widely spoken language in these states after English and Spanish (CNN World, 2014);

- Longitudinal studies should be considered that analyse the meanings of kinship for immigrant-host children (whether youth or adult) and the effects of VR travel on their individual and cultural identity as well as analysing their material and/or emotional interdependence with their relatives from overseas;

- From a feminist perspective, studies that examine the advantages and disadvantages for immigrant women who host relatives and friends (e.g., 
mobility in their new homeland; cultural norms or barriers to socialisation; career and/or educational benefits from receiving help with childcare);

- Studies could be undertaken that examine the valuation of the nonmonetary benefits and costs of having a VR to assist an immigrant-host family in times of need (e.g., taking care of an ill person; babysitting; elder care) and its implication for the social services and the healthcare system of their new homeland;

- Should studies be undertaken on a larger scale, a multi-disciplinary, multicultural, and multi-lingual team inquiring on the meanings of social interactions of the succeeding generations of immigrants and their respective VFRs may be required. Having both cultural insiders and outsiders conducting the study in order to counter the "bias" of the insider with the assumed "objectivity" of the outsider (Pe-Pua, 2006);

- In relation to any future methodological approaches, the use of technology such as virtual communication may be explored while being sensitive to the needs and capacity of the research participants (e.g., technologicallychallenged individuals such as elder people who may not be comfortable in using advanced technology). This researcher also acknowledges that any benefits of the use of virtual communications in any future research may be negated by their inherent limitations;

- There needs to be an enhancement of support that augments the capabilities of non-Western researchers and increased resources allocated to studies related to the social, cultural, or religious aspects of VFR travel and communicating them in both academic and non-academic settings;

- Consideration needs to be made on the future impacts on tourism and migration policies, and on technological advancement to VFR travel (e.g., virtual visa interviews and biometric identification);

- In general, more research should be undertaken both from a non-Western and Western perspectives that examines the social interactions between hosts and guests in the context of VFR travel. Very little research has been conducted about the meanings and understandings of the interactions from the perspective of both the hosting and visiting groups. 
Consequently, the conceptual framework that was used in this study may be reframed using other sociological and cultural theories while incorporating the indigenous philosophies of other scholars for interpreting the meanings of the social interactions underpinning VFR travel in their own terms.

\subsection{Conclusion: immigrant-hosts and VFRs - a kaleidoscope of social interactions}

Host-guest interactions in the context of VFR travel as a social phenomenon are multifaceted, complex, and dynamic. This study is based on the application of hermeneutic phenomenology for understanding and interpreting the social interactions between immigrant-hosts and their visiting friends and relatives at the micro-level. Metaphorically speaking, illuminating the relationships between immigrant-hosts and VFRs presents a myriad of nuances and are found to be dynamic, much like looking through a turning kaleidoscope. The interactions of the immigrant-hosts and VFRs during the visit were recounted in the interviews as complementary and contrasting perspectives which were provided through the different voices of the hosts and guests. Social interactions are therefore multidimensional as they involve various actors which the study was able to bring together to form its meanings or interpretations both individually and collectively.

The conceptualisation of the interactions of hosts and guests in the context of VFR travel also reflects aspects of a kaleidoscope of social interactions that unfold where the pattern (or prism) may use different theoretical lenses. Utilising social exchange theory and the theory of emotional solidarity when interpreting the interactions between immigrant-hosts and VFRs, the conceptualisation was reframed as previous studies on social interactions were based on the encounters or meeting of "strangers." In contrast, this research focuses on hosts and guests who have a shared culture and existing family or friendship ties. Also, this thesis explores social interactions where the participants take on host and guest roles which are layered upon other elements of their pre-existing relationships. This study recognises that the hosts and guests may share a similar cultural background, while the social interactions are taking place in another cultural setting. 
Considering that this research is based on immigrant-host communities together with their respective VFRs from their former homeland, Western concepts and indigenous philosophies may be harmonised to interpret the social interactions. While recognising that the researcher is an "outsider" to the culture of New Zealand and an "insider" to the culture of the participants' shared homeland, this study articulates the interpretations of the social interactions between Filipino immigrant-hosts and VFRs which the tourism industry and other sectors may benefit in the production and consumption of experiences, both for immigrant communities and their VFRs. Additionally, this study has laid the groundwork for other non-Western tourism scholars to examine the interpretations of friendship and kinship in the context of VFR travel in other settings.

This study is limited in scope by being situated within the social interactions of firstgeneration Filipino immigrants and their VFRs from the Philippines and is based on a small number of hosting and visiting friends and family members. More research is needed across different settings to accommodate the complexity of immigrant-host families (single parents, gay/lesbian families, step relations, extended families, and multi-ethnic families) who are hosting in the context of VFR travel while acknowledging the methodological and ethical implications and limitations. VFR travel is distinct in that it entails more than traditional economic exchanges as the roles and implications of family and friendship networks through social interactions remains underestimated. The future scope of VFR travel goes beyond the study of its economic contribution. Going forward, research should focus on understanding the motivations and benefits of maintaining social relationships between friends and families where "being with others" is both nurtured and fostered across time, space, and generations. 


\section{References}

ABS-CBN. (2014). Cebu Pacific eyes flights to New Zealand. Retrieved May 30, 2014, from http://www.abs-cbnnews.com/business/04/21/14/cebu-pacific-eyesflights-new-zealand

Adam, B. D. (2013). Queer company: the role and meaning of friendship in gay men's work lives. Contemporary Sociology: A Journal of Reviews, 42(1), 103-104.

Adams, R. G., \& Blieszner, R. (1994). An integrative conceptual framework for friendship research. Journal of Social and Personal Relationships(11), 163-184.

Aguilar, F. (2009). Labour migration and ties of relatedness: diasporic houses and investments in memory in a rural Philippine village. Thesis Eleven, 98(1), 8814.

Alampay, L. P., \& Jocson, M. R. M. (2011). Attitudes and attributes of mothers and fathers in the Philippines. Parenting: Science and Practice, 11(2-3), 163-176.

Allan, G. (1989). Friendship: developing a sociological perspective. Hertfordshire, UK: Harvester Wheatsheaf.

Altbach, P. G., \& Levy, D. C. (Eds.). (2005). Private higher education: a global revolution. Rotterdam, Netherlands: Sense Publishers.

Anderson, S. M., \& Chen, S. (2002). The relational self: an interpersonal socialcognitive theory. Psychological Review, 104(4), 619-645.

Andres, T. D. (1981). Understanding Filipino values: a management approach. Quezon City, Philippines: New Day.

Andres, T. D. (Ed.) (1994) Dictionary of Filipino culture and values. Quezon City: Giraffe Books.

Annis, D. B. (1987). The meaning, value, and duties of friendship. American Philosophical Quarterly, 24(4), 349-356.

Ap, J. (1992). Residents' perceptions on tourism impacts. Annals of Tourism Research, 19(4), 665-690.

Aramberri, J. (2001). The host should get lost: paradigms in the tourism theory. Annals of Tourism Research, 28(3), 738-761.

Aronson, J. (1994). A pragmatic view of thematic analysis. The Qualitative Report, 2(1).

Asiedu, A. B. (2008). Participants' characteristics and economic benefits of visiting friends and relatives (VFR) tourism - an international survey of the literature with implications for Ghana. International Journal of Tourism Research, 10, 609-621. 
Astedt-Kurki, P., Paavilainen, E., \& Lehti, K. (2001). Methodological issues in interviewing families in family nursing research. Journal of Advanced Nursing, 35(2), 288-293.

Babor, E. R. (2007). Heidegger's philosophy of authentic existence and the Filipinos: social dimension (Part II). KINAADMAN An Interdisciplinary Research Journal, 18(2), 1-9.

Backer, E. R. (2007). VFR travel - an examination of the expenditures of VFR travellers and their hosts. Current Issues in Tourism, 10(4), 366-377.

Backer, E. R. (2008). VFR Travellers - Visiting the Destination or Visiting the Hosts? Asian Journal of Tourism and Hospitality Research, 2(1), 60-70.

Backer, E. R. (2010a). The relationship of VFR travellers with tourism industries. Paper presented at the CAUTHE 2010: Tourism and Hospitality: Challenge the Limits.

Backer, E. R. (2010b). VFR travel: an assessment of VFR versus non-VFR travellers. (Doctor of Philosophy), Southern Cross University, Lismore, NSW. Retrieved from http://epubs.scu.edu.au/cgi/viewcontent.cgi?article=1126\&context=theses

Backer, E. R. (2012a). VFR travel: it is underestimated. Tourism Management, 33, 7479.

Backer, E. R. (2012b). VFR Travel: why marketing to Aunt Betty matters. In H. Schänzel, I. Yeoman \& E. Backer (Eds.), Family Tourism (pp. 81-92). Bristol, UK: Channel View.

Bǣrenholdt, J. O., Haldrup, M., Larsen, J., \& Urry, J. (2004). Performing tourist places. Hants, UK: Ashgate.

Bailey, K. (1994). Typologies and taxonomies: an introduction to classification techniques (Sage University paper series on quantitative applications in the social sciences, series no. 07-102). Thousand Oaks: SAGE.

Barber, P. G. (2000). Agency in Philippine women's labour migration and provisional diaspora. Women's Studies International Forum, 23(4), 399-411.

Batan, C. M. (2010). Istambay: a sociological analysis of youth inactivity in the Philippines. (Doctor of Philosophy), Dalhousie University, Halifax, Nova Scotia.

Beitin, B. K. (2008). Qualitative research in marriage and family therapy: who is in the interview? Contemporary Family Therapy, 30, 48-58.

Bell, S., \& Coleman, S. (Eds.). (1999). The Anthropology of Friendship. Oxford: Berg.

Bengtson, V. L., \& Schrader, S. S. (1982). Parent-child relations. In D. J. Mangen \& W. A. Peterson (Eds.), Handbook of research instruments in social gerontology (Vol. 2, pp. 115-185). Minneapolis: University of Minnesota Press. 
Blau, P. M. (1964). Exchange and power in social life. New York: John Wiley \& Sons, Inc.

Blau, P. M. (1986). Exchange and power in social life. New Brunswick, NJ: Transaction Books.

Blieszner, R., \& Adam, R. G. (1992). Adult friendship. Newbury Park, CA: SAGE.

Bolaffi, G., Bracalenti, R., Braham, P., \& Gindro, S. (2003). Family. Dictionary of race, ethnicity and culture (pp. 115-117). London: SAGE.

Bonus, R. (2000). Filipino Americans: ethnicity and the cultural politics of space. Philadelphia: Temple University Press.

Boyne, S., Carswell, F., \& Hall, D. (2002). Reconceptualising VFR: friends, relatives and migration in a domestic context. In C. M. Hall \& A. M. Williams (Eds.), Tourism and migration: new relationships between production and consumption (pp. 241-256). Dordrecht, The Netherlands: Kluwer Academic Publishers.

Bradley, G. (2014). Cebu Pacific considers NZ route. Retrieved May 30, 2014, from http://www.nzherald.co.nz/business/news/article.cfm?c id=3\&objectid=1124 $\underline{2443}$

Brocx, M. (2003). Visits by friends and relatives revisited: a study of host behaviour in Auckland, New Zealand. (Master of Tourism Management), Victoria University of Wellington, Wellington.

Brown, K. G. (2010). Come on home: visiting friends and relatives - the Cape Breton experience. Event Management, 14, 309-318.

Brzeski, V. J., Graham, J., \& Newkirk, G. F. (2001). Participatory Research and CBCRM: In Context: Coastal Resources Research Network (CoRR) Dalhousie University, Halifax, Nova Scotia, Canada and International Development Research Centre (IDRC), Ottawa, Ontario, Canada.

Bulbeck, C. (1998). Re-orienting western feminisms: women's diversity in a postcolonial world. New York: Cambridge University Press.

Burawoy, M. (1998). The extended case method. Sociological Theory, 16(1), 4-33.

Byrd, E. T., Bosley, H. E., \& Dronberger, M. G. (2009). Comparisons of stakeholder perceptions of tourism impacts in rural eastern North Carolina. Tourism Management, 30(5), 693-703.

Capistrano, R. C. G. (2013). Visiting friends and relatives travel, host-guest interactions and qualitative research: methodological and ethical implications. Asia-Pacific Journal of Innovation in Hospitality and Tourism, 2(1), 87-100.

Carr, N. (2011). Children's and families' holiday experiences. New York: Routledge.

Carr, S. C. (Ed.). (2010). The Psychology of Global Mobility. New York: Springer. 
Castagno, A. E. (2012). What makes critical ethnography "critical"? In S. D. Lapan, M. T. Quartaroli \& F. J. Riemer (Eds.), Qualitative research: an introduction to methods and designs (pp. 373-390). San Francisco: Jossey-Bass.

Causevic, S., \& Lynch, P. (2009). Hospitality as a human phenomenon: host-guest relationships in a post-conflict setting. Tourism and Hospitality Planning and Development, 6(2), 121-132.

Cederholm, E. A. (2004). The Use of Photo-elicitation in Tourism Research - Framing the Backpacker Experience. Scandinavian Journal of Hospitality and Tourism, $4(3), 225-241$.

Chesla, C. A. (1995). Hermeneutic phenomenology: an approach to understanding families. Journal of Family Nursing, 1(1), 63-78.

Chibucos, T. R., Leite, R. W., \& Weis, D. L. (Eds.). (2005). Readings in family theory. Thousand Oaks, California: SAGE.

Church, A. T. (1987). Personality research in a non-Western culture: the Philippines. Psychological Bulletin, 102(2), 272-292.

Church, A. T., Katigbak, M. S., \& Castaneda, I. (1988). The effects of language of data collection on derived conceptions of healthy personality with Filipino bilinguals. Journal of Cross-Cultural Psychology, 19(2), 178-192.

Citizen and Immigration Canada. (2013). The parent and grandparent super visa. Retrieved June 1, 2014, from http://www.cic.gc.ca/english/visit/supervisawho.asp

CNN World. (2014). America's linguistic melting pot. Retrieved May 30, 2014, from http://globalpublicsquare.blogs.cnn.com/2014/05/20/americas-linguisticmelting-pot/

Coles, T., Duval, D., \& Hall, C. M. (2004). Tourism, mobility and global communities: new approaches to theorising tourism and tourist spaces. In W. Theobold (Ed.), Global Tourism: The Next Decade (pp. 463-481). Oxford: Butterworth Heinemann.

Coles, T., \& Timothy, D. J. (Eds.). (2004). Tourism, diasporas and space. London: Routledge.

Collett, J. L. (2010). Integrating theory, enhancing understanding: the potential contributions of recent experimental research in social exchange for studying intimate relationships. Journal of Family Theory and Review, 2, 280-298.

Cook, K. S., \& Rice, E. (2003). Social exchange theory. In J. Delamater (Ed.), Handbook of Social Psychology (pp. 53-76). New York: Kluwer.

Corbin, J., \& Strauss, A. (2008). Basics of qualitative research: techniques and procedures for developing grounded theory (3rd ed.). Los Angeles: SAGE. 
Coventry, N. (2014a). New NZ/Filipino traffic rights - just in case. Inside Tourism, 968, 14.

Coventry, N. (2014b). NZ must become more multi-lingual says Goff. Inside Tourism, 979, 14.

Crompton, J. L. (2005). Issues related to sustaining a long-term research interest in tourism. Journal of Tourism Studies, 16(2), 34-43.

Cropanzano, R., \& Mitchell, M. S. (2005). Social exchange theory: an interdisciplinary review. Journal of Management, 31(6), 874-900.

Cunningham, J. W., \& Fitzgerald, J. (1996). Epistemology and reading. Reading Research Quarterly, 31(1), 36-60.

Cutler, S. Q., \& Carmichael, B. A. (2010). The dimensions of the tourist experience. In M. Morgan, P. Lugosi \& R. Brent (Eds.), The Tourism and Leisure Experience (pp. 3-26). Bristol: Channel View.

Dandaneau, S. P. (2007). Norms. In G. Ritzer (Ed.), The Blackwell Encyclopedia of Sociology (Vol. 7, pp. 3229-3232). Oxford: Blackwell Publishing Ltd.

Dann, G. (Ed.). (2002). The tourist as a metaphor of the social world. Oxon, UK: CABI.

Dann, G., \& Cohen, E. (1991). Sociology and tourism. Annals of Tourism Research, 18, 155-169.

De Castro, L. (2001). Debts of goodwill and interpersonal justice. http://www.bu.edu/wcp/Papers/Asia/AsiaDeCa.htm

de Vries, B. (1996). The understanding of friendship: an adult life course perspective. In C. Magai \& S. H. McFadden (Eds.), Handbook of Emotion, Adult Development, and Aging (pp. 249-268). San Diego, California: Academic Press.

Deaux, K., Reid, A., Mizrahi, K., \& Ethier, K. A. (1995). Parameters of social identity. Journal of Peronality and Social Psychology, 68(2), 280-291.

Deloso, L. S. (2007). Understanding the grounds of debts of goodwill, a Filipino concept of "utang na loob". KINAADMAN An Interdisciplinary Research Journal, 18(2), 1-10.

Denzin, N. K., \& Lincoln, Y. S. (1998). The art of interpretation, evaluation, and presentation. In N. K. Denzin \& Y. S. Lincoln (Eds.), Collecting and interpreting qualitative materials (pp. 275-281). Thousand Oaks, CA: SAGE.

Department of Labour. (2011). International migration outlook - New Zealand 2010/11: Labour and Immigration Research Centre, Department of Labour.

Derlega, V. J., \& Winstead, B. A. (Eds.). (1986). Friendship and social interaction. New York: Springer-Verlag. 
Dilworth-Anderson, P., Burton, L., \& Johnson, L. (2009). Reframing theories for understanding race, ethnicity, and families. In P. Boss, W. Doherty, R. LaRossa, W. Schumm \& S. Steinmetz (Eds.), Sourcebook of Family Theories and Methods (pp. 627-646). New York: Springer.

Donmoyer, R. (1990). Generalizability and the single case study. In E. Eisner \& A. Peshkin (Eds.), Qualitative inquiry in education: the continuing debate. New York: Teachers College Press.

Dreher, J. (2012). Investigating friendship: a prospective dispute between protosociology and phenomenological sociology. In N. Hisashi, F. C. Waksler \& C. Papadimitriou (Eds.), Interaction and everyday life: phenomenological and ethnomethodological essays in honor of George Psathas. Blue Ridge Summit, PA: Lexington Books.

Duncan, J., \& Lambert, D. (2003). Landscapes of home. In J. Duncan, N. Johnson \& R. Schein (Eds.), A Companion to Cultural Geography (pp. 382-403). London: Blackwell.

Durkheim, E. (1915/1995). The elementary forms of the religious life. New York: Free Press.

Duval, D. (2003). When host becomes guest: return visits and diasporic identities in a Commonwealth eastern Carribean community. Current Issues in Tourism, 6(4), 267-308.

Dwyer, L., Seetaram, N., Forsyth, P., \& King, B. (2014). Is the migration-tourism relationship only about VFR? Annals of Tourism Research, 46, 130-143.

Eder, D., \& Fingerson, L. (2001). Interviewing Children and Adolescents. In J. F. Gubrium \& J. A. Holstein (Eds.), Handbook of Interview Research (pp. 181202). Thousand Oaks: SAGE.

Enriquez, V. G. (1992). From colonial to liberation psychology: the Philippine experience. Quezon City, Philippines: University of the Philippines Press.

Enriquez, V. G. (1993). Developing a Filipino psychology. Indigenous psychologies: Research and experience in cultural context (Vol. 17, pp. 152-169). Thousand Oaks, CA: SAGE.

Espiritu, Y. L. (2003). Home bound: Filipino American lives across cultures, communities, and countries. Los Angeles: University of California Press.

European Commission. (2014). List of airlines banned within the EU. Retrieved May 30, 2014, from http://ec.europa.eu/transport/modes/air/safety/airban/doc/list_en.pdf

Evans, A., Elford, J., \& Wiggins, D. (2008). Using the internet for qualitative research. In C. Willig \& W. Stainton-Rogers (Eds.), The SAGE Handbook of Qualitative Research in Psychology (pp. 315-333). London: SAGE. 
Eysenbach, G., \& Till, J. E. (2001). Ethical issues in qualitative research on internet communities. British Medical Journal, 323 (7321), 1103-1105.

Fields, J., Copp, M., \& Kleinman, S. (2006). Symbolic interactionism, inequality, and emotions. In J. E. Stets \& J. H. Turner (Eds.), Handbook of the Sociology of Emotions. Riverside, CA: Springer.

Fischler, C. (2011). Commensality, society, and culture. Social Science Information, 50(3-4), 528-548.

Fredline, E. (2005). Host and guest relations and sport tourism. Sport in Society, 8(2), 263-279.

Fridgen, J. D. (1984). Environmental psychology and tourism. Annals of Tourism Research, 11, 19-39.

Friedman, M. (1993). What are friends for? Feminist perspectives on personal relationships and moral theory. New York: Cornell University Press.

Gahagan, J. (1984). Social interaction and its management. London: Methuen.

Gaskell, G. (2003). Individual and group interviewing. In M. W. Bauer \& G. Gaskell (Eds.), Qualitative researching with text, image and sound (pp. 38-56). London: SAGE.

Germann Molz, J. (2005). Global abode: at home in the world, mimeo. Lancaster University: Department of Sociology.

Germann Molz, J. (2006). 'Watch us wander': mobile surveillance and the surveillance of mobility. Environment and Planning, 38, 377-393.

Gheasi, M., Nijkamp, P., \& Rietveld, P. (2011). Migration and tourist flows. In A. Matias (Ed.), Tourism economics: impact analysis (pp. 111-126). Berlin: Springer.

Gibbs, G. R. (2007). Analyzing qualitative data. London: SAGE.

Gibson, W. J., \& Brown, A. (2009). Working with qualitative data. London: SAGE Publications, Inc.

Gnoth, J. (1997). Tourism motivation and expectation formation. Annals of Tourism Research, 24(2), 283-304.

Goeldner, C., \& Ritchie, B. (2009). Tourism: principles, practices, philosophies (11th ed.). New Jersey: Wiley \& Sons.

Goffman, E. (1967). Interaction ritual: essays on face-to-face behavior. Garden City, NY: Anchor Books. 
Gomez, C., \& Rodriguez, J. (2006). Four keys to Chilean culture: authoritarianism, legalism, fatalism and compadrazgo. Asian Journal of Latin American Studies, 19(3), 43-65.

Gough, S. N. (2001). Five reasons for nonprofit organizations to be inclusive. New Directions for Philanthropic Fundraising(34), 101-118.

Goulding, C., \& Saren, M. (2010). Immersion, emergence and reflexivity: grounded theory and aesthetic consumption. International Journal of Culture, Tourism and Hospitality Research, 4(1), 70-82.

Griffin, T. (2012). Research note: a content analysis of articles on visiting friends and relatives tourism, 1990-2010. Journal of Hospitality Marketing and Management.

Griffin, T. (2013). A paradigmatic discussion for the study of immigrant hosts. Current Issues in Tourism. doi: DOI:10.1080/13683500.2012.755157

Gu, C.-J. (2010). Culture, emotional transnationalism and mental distress: family relations and well-being among Taiwanese immigrant women. Gender, Place \& Culture, 17(6), 687-704.

Guba, E. G., \& Lincoln, Y. S. (1981). Effective evaluation: improving the usefulness of evaluation results through responsive and naturalistic approaches. San Francisco: Jossey-Bass.

Guevara, J. P. (2005). Pakikipagkapwa (sharing/merging oneself with others). In R. M. Gripaldo (Ed.), Filipino cultural traits: Philippine philosophical studies (pp. 920). Washington: The Council for Research in Values and Philosophy.

Gunn, C. (1988). Vacationscape: designing tourist regions (2nd ed.). Austin: University of Texas.

Gyimóthy, S. (1999). Visitors' perceptions of holiday experiences and service providers: an exploratory study. Journal of Travel \& Tourism Marketing, 8(2), 57-74.

Hall, C. M. (2005). Reconsidering the geography of tourism and contemporary mobility. Geographical Research, 43(2), 125-139.

Hall, C. M. (2007). Response to Yeoman et al: the fakery of 'the authentic tourist'. Tourism Management, 28, 1139-1140.

Hall, C. M., \& Duval, D. (2000). Linking diasporas and tourism: transnational mobilities of Pacific islanders resident in New Zealand. In T. Coles \& D. J. Timothy (Eds.), Tourism, Diasporas and Space (pp. 78-94). London: Routledge.

Hammarstrom, G. (2005). The construct of intergenerational solidarity in a lineage perspective: a discussion on underlying theoretical assumptions. Journal of Aging Studies, 19(1), 33-51. 
Handel, G. (1992). The qualitative tradition in family research. In J. F. Gilgun, K. Daly \& G. Handel (Eds.), Qualitative Methods in Family Research (pp. 12-21). Newbury Park, CA: SAGE.

Hannam, K., Sheller, M., \& Urry, J. (2006). Editorial: mobilities, immobilities and moorings. Mobilities, 1(1), 1-22.

Harper, S. E. (2010). Exploring the role of Filipino fathers: paternal behaviors and child outcomes. Journal of Family Issues, 31(1), 66-89.

Heidegger, M. (1996). Being and time. A translation of Sein und Zeit (J. Stambaugh, Trans.). New York: University of New York Press.

Hernandez, S. A., Cohen, J., \& Garcia, H. (1996). Residents' attitudes towards an instant resort enclave. Annals of Tourism Research, 23(4), 755-779.

Heuman, D. (2005). Hospitality and reciprocity: working tourists in Dominica. Annals of Tourism Research, 32(2), 407-418.

Hibbert, J. F., Dickinson, J. E., \& Curtin, S. (2013). Understanding the influence of interpersonal relationships on identity and tourism travel. Anatolia: An International Journal of Tourism and Hospitality Research, 24(1), 30-39.

Hill, R. S. (2010). Fitting Multiculturalism into Biculturalism: Maori-Pasifika Relations in New Zealand from the 1960s. Ethnohistory, 57(2), 291-319.

Ho, D. Y. F. (1998). Indigenous psychologies: Asian perspectives. Journal of CrossCultural Psychology, 29, 88-103.

Hofstede, G. (2011). Dimensionalizing cultures: the Hofstede model in context. Online readings in psychology and culture Unit 2, Article 8. from http://scholarworks.gvsu.edu/cgi/viewcontent.cgi?article=1014\&context=orpc

Hollinshead, K. (2000). Other. In J. Jafari (Ed.), Encyclopedia of Tourism (pp. 420). London: Routledge.

Holstein, J. A., \& Gubrium, J. F. (2003). Interviewing older people. In J. A. Holstein \& J. F. Gubrium (Eds.), Inside Interviewing. Thousand Oaks, CA: SAGE.

Humble, Á. M. (2010). Families. In A. J. Mills, G. Durepos \& E. Wiebe (Eds.), Encyclopedia of case study research (pp. 394-396). London: SAGE.

Hung, K., Xiao, H., \& Yang, X. (2013). Why immigrants travel to their home places: social capital and acculturation perspective. Tourism Management, 36, 304313.

Huong, N. T., \& King, B. (2002). Migrant communities and tourism consumption: the case of the Vietnamese in Australia. In C. M. Hall \& A. M. Williams (Eds.), Tourism and Migration: New Relationships between Production and Consumption (pp. 221-240). Dordrecth, The Netherlands: Kluwer Academic Publishers. 
IIRR. (1998). Participatory methods in community-based coastal resource management (Vol. 1-3). Silang, Cavite: International Institute of Rural Reconstruction.

Immigration New Zealand. (2012). Information for relationship-based visa applications. May 6, 2013, from http://www.immigration.govt.nz/branch/manila/partnership/

Immigration New Zealand. (2013). About Immigration New Zealand. Retrieved May 30, 2014, from http://www.immigration.govt.nz/migrant/general/aboutnzis/

International Organization for Migration (IOM). (2011). Facts and figures. Retrieved February 16, 2012, from http://www.iom.int/jahia/Jahia/aboutmigration/facts-and-figures/lang/en

Jackson, R. (1990). VFR tourism: is it underestimated? . The Journal of Tourism Studies, 1(2), 10-17.

Jamal, T., \& Hollinshead, K. (2001). Tourism and the forbidden zone: the underserved power of qualitative inquiry. Tourism Management, 22, 63-82.

Jennings, G. R. (2009). Methodologies and methods. In T. Jamal \& M. Robinson (Eds.), The SAGE Handbook of Tourism Studies (pp. 672-692). London: SAGE.

Jennings, G. R. (2010). Tourism Research (2nd ed.). Queensland: John Wiley \& Sons Australia, Ltd.

Jones, P. S. (1995). Paying respect: care of elderly parents by Chinese and Filipino American women. Health Care for Women International, 16(5), 385-398.

Kagitcibasi, C. (2005). Autonomy and relatedness in cultural context: implications for Self and Family. Journal of Cross-Cultural Psychology, 36(4), 403-422.

Kataoka-Yahiro, M. R., Ceria, C., \& Yoder, M. (2004). Grandparent caregiving role in Filipino American families. Journal of Cultural Diversity, 11(3), 110-117.

Kenrick, D., Neuberg, S., \& Cialdini, R. (2010). Social psychology: goals in interaction. Boston: Pearson.

Kikuchi, Y. (2001). The social role of the Filipino ritual kinship system (through theoretical issues of cognatic kinship form). Waseda University.

Kinnaird, V., \& Hall, D. (1996). Understanding tourism processes: a gender-aware framework. Tourism Management, 17(2), 95-102.

Kvale, S. (2007). Doing interviews. London: SAGE.

Kvale, S., \& Brinkmann, S. (2009). InterViews: learning the craft of qualitative research interviewing. Los Angeles: SAGE. 
Lacy, J., \& Douglass, W. (2002). Beyond authenticity: the meaning and uses of cultural tourism. Tourist Studies, 2, 5-21.

Lai, D. (2010). Filial piety, caregiving appraisal, and caregiving burden. Research on aging, 32(2), 200-223.

Larsen, J. (2008). De-exoticizing tourist travel: everyday life and sociality on the move. Leisure Studies, 27(1), 21-34.

Larsen, J., Urry, J., \& Axhausen, K. W. (2007). Networks and tourism: mobile social life. Annals of Tourism Research, 34(1), 244-262.

Larsen, S. (2007). Aspects of a psychology of the tourist experience. Scandinavian Journal of Hospitality and Tourism, 7(1), 7-18.

Lashley, C., Lynch, P., \& Morrison, A. (2006). Ways of Knowing Hospitality. In C. Lashley, P. Lynch \& A. Morrison (Eds.), Advances in Tourism Research: Hospitality: A Social Lens. Amsterdam, Netherlands: Elsevier Science and Tecnology.

Laverty, S. M. (2003). Hermeneutic phenomenology and phenomenology: a comparison of historical and methodological considerations. International Journal of Qualitative Methods, 2(3), 1-28.

Lawler, E. J., \& Thye, S. R. (2006). Social exchange theory of emotions. In J. E. Stets \& J. H. Turner (Eds.), Handbook of the Sociology of Emotions (pp. 295-320). Riverside, CA: Springer.

Lawler, E. J., Thye, S. R., \& Yoon, J. (2000). Emotion and group cohesion in productive exchange. Journal of Sociology, 106(3), 616-657.

Lehto, X. Y., Morrison, A. M., \& O'Leary, J. T. (2001). Does the Visiting Friends and Relatives' typology make a difference? A study of the International VFR market to the United States. Journal of Travel Research, 40, 201-212.

Leiper, N. (1979). The framework of tourism: Towards a definition of tourism, tourist, and the tourist industry. Annals of Tourism Research, 6(4), 390-407.

Leiper, N. (2004). Tourism management (3rd ed.). Frenchs Forest: Pearson Education

Lévinas, E. (1991). Totality and infinity, an essay on exteriority. Dordrecht: Kluwer Academic Publishing.

Levitt, P., \& Jaworsky, N. B. (2007). Transnational migration studies: past developments and future trends. Annual Review of Sociology, 33, 129-156.

Levy, S. E. (2010). The hospitality of the host: a cross-cultural examination of managerially facilitated consumer-to-consumer interactions. International Journal of Hospitality Management, 29(2), 319-327. 
Lew, A., \& McKercher, B. (2006). Modeling tourist movements: a local destination analysis. Annals of Tourism Research, 33(2), 403-423.

Lincoln, Y. S., \& Guba, E. G. (1985). Naturalistic inquiry. Beverly Hills, CA: SAGE.

Lincoln, Y. S., \& Guba, E. G. (2000). Paradigmatic controversies, contradictions, and emerging confluences. In N. K. Denzin \& Y. S. Lincoln (Eds.), The Handbook of Qualitative Research (pp. 163-188). Thousand Oaks: SAGE.

Lockyer, T., \& Ryan, C. (2007). Visiting Friends and Relatives distinguishing between two groups: the case of Hamilton, New Zealand. Tourism Recreation Research, 32(1), 59-68.

Lowery, C. T. (2001). Ethnographic research methods. In B. A. Thyer (Ed.), The Handbook of Social Work Research Methods (pp. 320-331). Thousand Oaks: SAGE Publications, Inc.

Lynch, P., Molz, J. G., Mclntosh, A., Lugosi, P., \& Lashley, C. (2011). Editorial: Theorizing hospitality. Hospitality and Society Journal, 1-21.

MacCannell, D. (1976). The Tourist: A New Theory of the Leisure Class. New York: Schocken Books.

Macdonald, R. (2007). Pagbukadkad ng bulaklak= Blooming of the flower: an ethnographic study of a friendship group of same-sex attracted middle-class young men from Metro Manila in the Philippines. (Doctor of Philosophy), Edith Cowan University.

Marshall, C., \& Rossman, G. (2006). Designing qualitative research (4th ed.). Thousand Oaks: SAGE.

Martin, P., Abella, M., \& Midgley, E. (2004). Best practices to manage migration: the Philippines. International Migration Review, 38(4), 1544-1560.

Mazzucato, V. (2004). Transcending the nation: explorations of transnationalism as a concept and phenomenon. In D. Kalb, W. Panster \& H. Siebers (Eds.), Globalization and Development: Themes and Concepts in Current Research. Dordrecht, Netherlands: Kluwer Academic Publishers.

McKay, D. (2007). 'Sending dollars shows feeling' - emotions and economies in Filipino migration. Mobilities, 2(2), 175-194.

McKay, S. (2011) Re-masculinizing the hero: Filipino migrant men and gender privilege. Asia Research Institute Working Paper Series No. 172: Asia Research Institute - National University of Singapore.

McKercher, B. (1996). Host involvement in VFR travel. Annals of Tourism Research, 23(3), 701-703.

McKercher, B. (1999). A chaos approach to tourism. Tourism Management, 20(4), 425-434. 
McNaughton, D. (2006). The "host" as uninvited "guest": hospitality, violence, and tourism. Annals of Tourism Research, 33(3).

Medina, B. T. G. (2001). The Filipino family. Quezon City, Philippines: University of the Philippines Press.

Merriam, S. B., \& Associates (Eds.). (2002). Qualitative research in practice: examples for discussion and analysis. San Francisco, CA: Jossey-Bass.

Merriam, S. B., Johnson-Bailey, J., Lee, M.-Y., Kee, Y., Ntseane, G., \& Muhamad, M. (2001). Power and positionality: negotiating insider/outsider status within and across cultures. International Journal of Lifelong Education, 20(5), 405-416.

Merrill, D. M. (2011). When your children marry: how marriage changes relationships with sons and daughters Lanham, Maryland: Rowman \& Littlefield.

Mesquita, B. (2001). Emotions in collectivist and individualist contexts. Journal of Personality and Social Psychology, 80(1), 68-74.

Mesquita, B., \& Frijda, N. H. (1992). Cultural variations in emotions: a review. Psychological Bulletin, 112(2), 179-204.

Mill, R., \& Morrison, A. (2006). The Tourism System (5th ed.). USA: Kendell-Hunt.

Ministry of Business, Innovation and Employment (MBIE). (2013). Short-term overseas visitor arrivals (by country of last residence). Retrieved January 12, 2014, from http://www.med.govt.nz/sectors-industries/tourism/tourismresearch-data/international-travel/iva-key-data

Ministry of Economic Development. (2009). International Visitors. Retrieved August 15,2011 , from http://www.tourismresearch.govt.nz/Documents/International\%20Market\%2 OProfiles/Total\%20Profile.pdf

Miralao, V. (1997). The family, traditional values, and the sociocultural transformation of Philippine society. Philippine Sociological Review, 45, 189-215.

Molm, L. D. (1994). Dependence and risk: transforming the structure of social exchange. Social Psychology Quarterly, 57, 163-176.

Molm, L. D., Takahashi, N., \& Peterson, G. (2000). Risk and trust in social exchange: an experimental test of a classical proposition. American Journal of Sociology, 105(5), 1396-1427.

Morris, L. (1990). The Workings of the Household. Cambrdige: Polity Press.

Morrison, A. M., Hsieh, S., \& O'Leary, J. T. (1995). Segmenting the Visiting Friends and Relatives market by holiday activity participation. The Journal of Tourism Studies, 6(1), 48-63. 
Morrison, A. M., Woods, B., Pearce, P., Moscardo, G., \& Sung, H. H. (2000). Marketing to the visiting friends and relatives segment: an international analysis. Journal of Vacation Marketing, 6(2), 102-118.

Moscardo, G., Pearce, P., Morrison, A., Green, D., \& O'Leary, J. T. (2000). Developing a typology for understanding Visiting Friends and Relatives markets. Journal of Travel Research, 38, 251-259.

Moyle, B., Croy, G., \& Weiler, B. (2010). Tourism interaction on islands: the community and visitor social exchange. International Journal of Culture, Tourism, and Hospitality Research, 4(2), 95-107.

Murdock, G. P. (1949). Social structure. New York: The MacMillan Company.

Murphy, L. (2001). Exploring social interactions of backpackers. Annals of Tourism Research, 28(1), 50-67.

Narayan, K. (1993). How native is a "native" anthropologist. American Anthropologist, 95(3), 671-686.

Nardi, P. M., \& Sherrod, D. (1994). Friendship in the lives of gay men and lesbians. Journal of Social and Personal Relationships, 11(2), 185-199.

Nash, J., \& Calonico, J. M. (1996). The meanings of social interaction: an introduction to social psychology. New York: General Hall.

Navarro, R. L., \& Turco, D. (1994). Segmentation of the visiting friends and relatives travel market. Visions in leisure and business, 13(1), 4-16.

New Zealand Ministry of Foreign Affairs and Trade. (2011). Republic of the Philippines. Retrieved April 27, 2011, from http://www.mfat.govt.nz/Countries/Asia-South-andSoutheast/Philippines.php

O'Connor, P. (1992). Friendships between women: a critical review. New York: Guilford Press.

O'Gorman, K. D. (2010). The origins of hospitality and tourism. Oxford: Goodfellow Publishers Ltd.

Obrador, P. (2012). The place of the family in tourism research: domesticity and thick sociality by the pool. Annals of Tourism Research, 39(1), 401-420.

Obsequio-Go, M. E. (2003). The Role of the host in visiting friends and or relatives (VFR) trips to the Philippines. (Master of Tourism), University of Otago, Otago. Retrieved from http://www.business.otago.ac.nz/tourism/teaching/postgraduate teaching/ Masters\%20Profiles/Maria\%200bsequio-Go.pdf

Obsequio-Go, M. E., \& Duval, D. T. (2003). Return visits among Filipino migrants in Dunedin, New Zealand. Tourism Review International, 7, 51-55. 
Owens, E. (2007). Significant others. In G. Ritzer (Ed.), The Blackwell Encyclopedia of Sociology (Vol. 9, pp. 4320-4321). Oxford: Blackwell Publishing Ltd.

Palovic, Z., Kam, S., Janta, H., Cohen, S., \& Williams, A. (2014). Surrey think tank reconceptualising visiting friends and relatives (VFR) travel. Journal of Destination Marketing \& Management, 2(4), 266-268.

Paltridge, B. (2002). Thesis and dissertation writing: an examination of published advice and actual practice. English for Specific Purposes, 21, 125-143.

Papathanassis, A. (2012). Guest-to-guest interaction on board cruise ships: exploring social dynamics and the role of situational factors. Tourism Management, 33(5), 1148-1158.

Parreñas, R. S. (2001). Mothering from a distance: emotions, gender, and intergenerational relations in Filipino transnational families. Feminist Studies, 27(2), 361-390.

Patton, M. Q. (1982). Qualitative methods and approaches: what are they? New Directions for Institutional Research, 34, 3-15.

Patton, M. Q. (2002). Qualitative Research and Evaluation Methods. Thousand Oaks, California: Sage Publications, Inc.

Payne, G., \& Payne, J. (2004). Reflexivity: SAGE Publications, Ltd.

Pe-Pua, R. (2006). From decolonizing psychology to the development of a crossindigenous perspective in methodology: the Philippine experience. In U. Kim, K.-S. Yang \& K.-K. Hwang (Eds.), Indigenous and Cultural Psychology (pp. 109137). New York: Springer Science+Business Media, Inc.

Pe-Pua, R., \& Protacio-Marcelino, E. (2000). Sikolohiyang Pilipino (Filipino psychology): a legacy of Virgilio G. Enriquez. Asian Journal of Social Psychology, 3, 49-71.

Pearce, D. G. (2012). Frameworks for Tourism Research. Oxfordshire: CABI.

Pearce, D. G. (2014). The internationalization of tourism research. Journal of Travel Research, 53(3), 267-280.

Pearce, P., \& Moscardo, G. (2005). Domestic and visiting friends and relatives tourism. In D. Buhalis \& C. Costa (Eds.), Tourism Business Frontiers: Consumes, Products and Industry (pp. 48-55). Jordan Hill, UK: Butterworth-Heinemann.

Pearce, P. L. (1984). Tourist-guide interaction. Annals of Tourism Research, 11(1), 129146.

Pearce, P. L. (1993). Fundamentals of tourist motivation. In D. G. Pearce \& R. W. Butler (Eds.), Tourism Research: Critiques and Challenges. London: Routledge. 
Pearce, P. L. (2005). Tourists Behaviour: Themes and Conceptual Schemes. Clevedon, UK: Channel View.

Pernecky, T., \& Jamal, T. (2010). (Hermeneutic) phenomenology in tourism studies. Annals of Tourism Research, 37(4), 1055-1075.

Pijpers, R., \& Maas, M. (2013). Identity construction and 'coincidental' entrepreneurship among gay Filipino guesthouse owners in Amsterdam. Gender, Place \& Culture: A Journal of Feminist Geography. doi: 10.1080/0966369X.2013.817971

Pilardi, J. (2010). Domestic hospitality: self, other, and community. In M. Hamington (Ed.), Feminism and Hospitality: Gender in the Host/Guest relationship (pp. 3952). Maryland: Lexington Books.

Pizam, A., Uriely, N., \& Reichel, A. (2000). The intensity of tourist-host social relationship and its effects on satisfaction and change of attitudes: the case of working tourists in Israel. Tourism Management, 21(4), 395-406.

Pons, P. O. (2003). Being-on-holiday: tourist dwelling, bodies and place. Tourist Studies, 3(1), 47-66.

Porter, E. J., \& Cohen, M. Z. (2013). Phenomenology. In A. A. Trainor \& E. Graue (Eds.), Reviewing Qualitative Research in the Social Sciences (pp. 180-196). New York: Routledge.

Pratt, G. (2003). Between homes: displacement and belonging for second-generation Filipino-Canadian youths. BC Studies, 140.

Prentice, R. C., Witt, S. F., \& Hamer, C. (1998). Tourism as experience: the case of heritage parks. Annals of Tourism Research, 25(1), 1-24.

Protacio-Marcelino, E. (1996). Identidad at etnisidad: pananaw at karanasan ng mga estudyanteng Filipino-Amerikano sa California (Identity and ethnicity: perspectives and experiences of Filipino-American students in California). (Doctor of Philosophy in Psychology), University of the Philippines, Quezon City, Philippines.

Quintin, T. A. D. (1981). Understanding Filipino values: a management approach. Quezon City: New Day Publishers.

Rawlins, W. K. (1999). Friendship. In D. Levinson, J. J. Ponzetti Jr. \& P. F. Jorgensen (Eds.), Encyclopedia of Human Emotions (Vol. 1). New York: Macmillan Referencw.

Reisinger, Y., \& Turner, L. W. (2003). Cross-Cultural Behaviour in Tourism: Concepts and Analysis. Oxford: Butterworth-Heinemann.

Reuters. (2014). United States upgrades Philippine civil aviation rating. Retrieved May 30, 2014, from http://www.reuters.com/article/2014/04/10/usphilippines-aviation-idUSBREA390VY20140410 
Riley, M. (2012). Emplacing the research encounter: exploring farm life histories. In J. Goodwin (Ed.), SAGE Biographical Research (Vol. 2, pp. 239-259). London: SAGE.

Riley, R. W., \& Love, L. L. (2000). The State of Qualitative Tourism Research. Annals of Tourism Research, 27(1), 164-187.

Ritzer, G. (Ed.). (2005). Encyclopedia of social theory. Thousand Oaks, California: SAGE.

Robben, A. C. G. M. (2007). Multi-sited fieldwork. Ethnographic Fieldwork: An Anthropological Reader (pp. 331-335). Oxford: Blackwell Publishing.

Robben, A. C. G. M., \& Sluka, J. A. (Eds.). (2007). Ethnographic Fieldwork: An Anthropological Reader. Oxford: Blackwell Publishing.

Roberts, R. E. L., Richards, L. N., \& Bengtson, V. L. (1991). Intergenerational solidarity in families: untangling the ties that bind. In S. K. Pfeifer \& M. B. Sussman (Eds.), Families: intergenerational and generational connections, Part One (pp. 11-46). Binghamton, N.Y.: Haworth.

Robinson, M., \& Lynch, P. (2006). The power of hospitality: a sociolingustic analysis. In C. Lashley, P. Lynch \& A. Morrison (Eds.), Advances in Tourism Research: Hospitality: A Social Lens. . Amsterdam, Netherlands: Elsevier Science and Tecnology.

Root, M. P. P. (Ed.). (1997). Filipino Americans: transformation and identity. Thousand Oaks: SAGE.

Rubio, R. J., \& Green, R.-J. (2009). Filipino masculinity and psychological distress: a preliminary comparison between gay and heterosexual men. Sexuality Research and Social Policy, 6(3), 61-75.

Rummel, R. J. (1976). Understanding conflict and war (Vol. 2). New York: SAGE.

Russo, M. T. (2014). Home, domesticity and hospitality: a theoretical reflection. Hospitality and Society, 2(3), 309-320.

Ryan, C. (2002). Motives, behaviours, body and mind. In C. Ryan (Ed.), The Tourist Experience (2nd ed., pp. 26-57). London: Continuum.

Ryley, T. J., \& Zanni, A. M. (2013). An examination of the relationship between social interactions and travel uncertainty. Journal of Transport Geography, 31, 249257.

Sandelowski, M., \& Barroso, J. (2007). Handbook for Synthesizing Qualitative Research. New York: Springer Publishing Company.

Santich, B. (2006). Hospitality and gastronomy: natural allies. In C. Lashley, P. Lynch \& A. Morrison (Eds.), Advances in Tourism Research: Hospitality: A Social Lens (pp. 47-59). London: Routledge. 
Sattar, Z., Hannam, K., \& Ali, N. (2013). Religious obligations to travel: first-generation Pakistani migrants from Newcastle-upon-Tyne. Journal of Tourism and Cultural Change.

Schänzel, H. A. (2010a). Family time and own time on holiday: generation, gender, and group dynamic perspectives from New Zealand. (Doctor of Philosophy in Tourism Management), Victoria University of Wellington, Wellington, NZ.

Schänzel, H. A. (2010b). Whole-family research: towards a methodology in tourism for encompassing generation, gender, and group dynamic perspectives. Tourism Analysis, 15, 559-569.

Schänzel, H. A. (2013). The importance of 'social' in family tourism. Asia-Pacific Journal of Innovation in Hospitality and Tourism, 2(1), 1-15.

Schänzel, H. A., \& Brocx, M. (2013). VFR travel: The host experience of the Polynesian community in Auckland [online]. Paper presented at the CAUTHE 2013: Tourism and Global Change: On the Edge of Something Big, Christchurch, N.Z.: Lincoln University.

Schänzel, H. A., Brocx, M., \& Sadaraka, L. (2014). (Un)conditional hospitality: the host experience of the Polynesian community in Auckland. Hospitality and Society, $4(2), 135-154$.

Schänzel, H. A., Smith, K. A., \& Weaver, A. (2005). Family holidays: a research review and application to New Zealand. Annals of Leisure Research, 8(2-3), 105-123.

Schänzel, H. A., Yeoman, I., \& Backer, E. (Eds.). (2012). Family Tourism: Multidisciplinary Perspectives. Bristol: Channel View.

Scheyvens, R. (2007). Poor cousins no more: valuing the development potential of domestic and diaspora tourism. Progress in Development Studies, 7(4), 307325.

Seaton, A. V. (1994). Are relatives friends? Reassessing the VFR category in segmenting tourism markets. In A. V. Seaton, C. L. Jenkins, R. C. Wood, P. U. C. Dieke, M. M. Bennett, L. R. MacLellan \& R. Smith (Eds.), Tourism: The State of the Art (pp. 316-321). Chichester, UK: John Wiley \& Sons, Ltd.

Seaton, A. V., \& Tagg, S. J. (1995). Disaggregating friends and relatives in VFR tourism research: the Northern Ireland evidence 1991-1993. Journal of Tourism Studies, 6(1), 6-18.

Shani, A. (2013). The VFR experience: 'home' away from home? Current Issues in Tourism, 16(1), 1-15.

Shani, A., \& Uriely, N. (2011). VFR tourism: the host experience. Annals of Tourism Research, 39(1), 421-440.

Sharp, R. (1982). Self-contained ethnography or a science of phenomenal forms and inner relations. Journal of Education, 164(1), 48-63. 
Sheller, M., \& Urry, J. (2006). The new mobilities paradigm. Environment and Planning, 38, 207-226.

Sheller, M., \& Urry, J. (Eds.). (2004). Tourism Mobilities: Places to Play, Places in Play. London: Routledge.

Sherlock, K. (2001). Revisiting the concept of hosts and guests. Tourist Studies, 1(3), 271-295.

Silverstein, M., \& Bengtson, V. L. (1997). Intergenerational solidarity and the structure of adult child-parent relationships in American families. American Journal of Sociology, 103(2), 429-460.

Skrbiš, Z. (2008). Transnational Families: Theorising Migration, Emotions and Belonging. Journal of Intercultural Studies, 29(3), 231-246.

Slater, K. C. (2002). Guess what we have planned for you today?: The influence of resident/hosts on the visitation activities of their visiting friends and relatives. (Master's thesis), Victoria University of Wellington, Wellington.

Smith, A. (1976). The theory of moral sentiments. Oxford, 1976, originally published in 1759: Oxford University Press.

Smith, V. L. (1977). Hosts and Guests: The Anthropology of Tourism. Philadelphia, PA: The University of Pennsylvania Press.

Smith, V. L. (Ed.). (1989). Hosts and Guests: The Anthropology of Tourism. Philadelphia: University of Pennsylvania Press.

Smith, V. L., \& Brent, M. (Eds.). (2001). Host and Guests Revisited: Tourism Issues of the 21st Century. New York: Cognizant Communication Corporation.

Snow, N. E. (2010). Hospitableness: a neglected virtue. In M. Hamington (Ed.), Feminism and hospitality: gender in the host/guest relationship (pp. 3-17). Lanham, Maryland: Lexington Books.

Sobritchea, C. I. (2012). The ideology of female domesticity: its impact on the status of Filipino women. Review of Women's Studies, 1(1), 26-41.

Song, M. (2006). Contextualization and discipleship: closing the gap between theory and practice. Evangelical Review of Theology, 30(3), 249-263.

Sontillanosa, R. (Cartographer). (2013). Key provinces and key cities within Metro Manila covered during the interviews in the Philippines.

Statistics New Zealand. (2007). Permanent and long-term migration from the Philippines. 2007: Statistics New Zealand.

Statistics New Zealand. (2014a). 2013 Census QuickStats about culture and identity. Wellington: Statistics New Zealand. 
Statistics New Zealand. (2014b). International travel and migration: April 2014.

Steiner, C. J., \& Reisinger, Y. (2006). Understanding existential authenticity. Annals of Tourism Research, 33(2), 299-318. doi: doi: 10.1016/j.annals.2005.08.002

Stets, J. E., \& Turner, J. H. (Eds.). (2006). Handbook of the Sociology of Emotions. Riverside, CA: Springer.

Stringer, P. F., \& Pearce, P. L. (1984). Toward a symbiosis of social psychology and tourism studies. Annals of Tourism Research, 11(1), 5-17.

Taylor, J. P. (2001). Authenticity and sincerity in tourism. Annals of Tourism Research, 28(1), 7-26.

Telfer, E. (1996). Food for thought: philosophy and food. London: Routledge.

Temple, B. (2005). Nice and tidy: translation and representation. Sociological Research Online, 10(2).

The New Zealand Herald. (2013). Census 2013: More ethnicities than the world's countries. Retrieved October 10, 2014, from http://www.nzherald.co.nz/nz/news/article.cfm?c id=1\&objectid=11170288

Thomas, J., \& Harden, A. (2008). Methods for the thematic synthesis of qualitative research in systematic views. BMC Medical Research Methodology, 8, 1-10.

Torre, B. A., Manalastas, E. J., Sese, D. G. A., \& Catanghal, A. (2005). Fag Hags in Filipino Gay Culture: Friendships, Identities, and Personality. Paper presented at the Sexualities, Genders, and Rights in Asia: The 1st International Conference of Asian Queer Studies" in Bangkok, Thailand.

Triandis, H. C. (1989). The self and social behavior in differing cultural contexts. Psychological Review, 96(3), 506-520.

Tribe, J. (2004). Knowing about tourism: epistemological issues. In J. Phillimore \& L. Goodson (Eds.), Qualitative Research in Tourism: Ontologies, Epistemologies, Methodologies (pp. 46-62). London: Routledge.

Tribe, J. (2006). The truth abouth tourism. Annals of Tourism Research, 33(2), 360381.

Tribe, J., \& Xiao, H. (2011). Developments in tourism social science. Annals of Tourism Research, 38(1), 7-26.

Tung, V. W. S., \& Ritchie, J. R. B. (2011). Exploring the essence of memorable tourism experiences. Annals of Tourism Research, 38(4), 1367-1386.

Turner, B. S. (2006) The Cambridge Dictionary of Sociology. Cambridge: Cambridge University Press. 
Turner, J. H. (1988). A theory of social interaction. Stanford, CA: Stanford University Press.

United Nations Development Programme. (2009). Human Development Report 2009, Overcoming barriers: Human Mobility and Development. New York: United Nations Development Programme,.

Uriely, N. (2005). The tourist experience: conceptual developments. Annals of Tourism Research, 32(1), 199-216.

Urry, J. (2002a). Mobility and proximity. Sociology, 36, 255-274.

Urry, J. (2002b). The Tourist Gaze (2nd ed.). London: SAGE.

Urry, J. (2003). Social networks, travel and talk. British Journal of Sociology, 54(2), 155-176.

Uttal, L. (1999). Using kin for child care: embedment in the socioeconomic networks of extended families. Journal of Marriage and Family, 61(4), 845-857.

Van Manen, M. (1990). Researching Lived Experience: Human Science for an Action Sensitive Pedagogy. New York: State University of New York.

Velasco, P. (2002). Filipino migrant workers amidst globalization Canadian Woman Studies, 21(4), 131-135.

Villacorta, W. V. (2002). Duties of children towards the elderly: East Asian perspectives on intergenerational solidarity. Paper presented at the Eight Plenary Session of the Pontifical Academy of Social Sciences, Vatican City.

Wallace, R. A., \& Hartley, S. F. (1988). Religious elements in friendship: Durkheimian theory in an empirical context. In J. C. Alexander (Ed.), Durkheimian sociology: cultural studies (pp. 93-106). Cambridge: Cambridge University Press.

Wallace, R. A., \& Wolf, A. (2006). Contemporary Sociological Theory: Expanding the Classical Tradition (6th Ed.). Upper Saddle River, NJ: Pearson.

Wang, N. (1999). Rethinking Authenticity In Tourism Experience. Annals of Tourism Research, 26(2), 349-370.

Ward, C., \& Berno, T. (2011). Beyond social exchange theory: Attitudes Toward Tourists. Annals of Tourism Research, 38(4), 1556-1569.

Watkins, L., \& Gnoth, J. (2011). The value orientation approach to understanding culture. Annals of Tourism Research, 38(4), 1247-1299.

White, N. R., \& White, P. B. (2008). Travel as interaction: encountering place and others. Journal of Hospitality \& Tourism Management, 15(3), 42-48.

Williams, A. M., \& Hall, C. M. (2000). Tourism and migration: new relationships between production and consumption. Tourism Geographies, 2(1), 5-27. 
Williams, A. M., \& Hall, C. M. (2002). Tourism, migration, circulation and mobility. In C. M. Hall \& A. M. Williams (Eds.), Tourism and Migration: New Relationships between Production and Consumption (pp. 1-52). Dordrecht, The Netherlands: Kluwer Academic Publishers.

Williams, S. (1998). Tourism Geography. London: Routledge.

Wiseman, J. P. (1986). Friendship: bonds and binds in a voluntary relationship. Journal of Social and Personal Relationships, 3, 191-211.

Wolf, D. L. (1997). Family secrets: transnational struggles among children of Filipino immigrants. Sociological Perspectives, 40(3), 457-482.

Wolf, D. L. (2002). There's No Place Like 'Home': Emotional Transnationalism and the Struggles of Second-Generation Filipinos. In P. Levitt \& M. C. Walters (Eds.), The Changing Face of Home: The Transnational Lives of the Second Generation (pp. 255-294). New York: Russell Sage Foundation.

Woosnam, K. M. (2011). Comparing residents' and tourists' emotional solidarity with one another: an extension of Durkheim's model. Journal of Travel Research, 50(6), 615-626.

Woosnam, K. M., \& Aleshinloye, K. D. (2012). Can tourists experience emotional solidarity with residents? Testing Durkheim's model from a new perspective. Journal of Travel Research, 52(4), 494-505.

Woosnam, K. M., \& Norman, W. C. (2009). Measuring residents' emotional solidarity with tourists: scale development of Durkheim's theoretical constructs. Journal of Travel Research, 49(3), 365-380.

Woosnam, K. M., Norman, W. C., \& Ying, T. (2009). Exploring the theoretical framework of emotional solidarity between residents and tourists. Journal of Travel Research, 48(2), 245-258.

Yeoman, I., Davis, J., Wheatley, C., Mars, M., Schänzel, H. A., \& Butterfield, S. (2012). Tourism2050: Scenarios for New Zealand. Wellington, New Zealand: Victoria University of Wellington.

Young, C. A., Corsun, D. L., \& Baloglu, S. (2007). A taxonomy of hosts visiting friends and relatives. Annals of Tourism Research, 34(2), 497-516.

Yu, J., \& Lee, T. J. (2014). Impact of tourists' intercultural interactions. Journal of Travel Research, 53(2), 225-238.

Zhang, J., Inbakaran, R. J., \& Jackson, M. S. (2006). Understanding community attitudes towards tourism and host-guest interaction in the urban-rural border region. Tourism Geographies, 8(2), 182-204. 


\section{List of Appendices}

Appendix 1. E-mail permission to the internet community moderator.

Appendix 2. E-mail announcement to the internet community.

Appendix 3. Information sheet for the immigrant-host family interviews in New Zealand.

Appendix 4. Immigrant-host family in New Zealand/visiting friends and felatives in the Philippines Consent Form.

Appendix 5. Individual interview guide for the immigrant-host family in New Zealand.

Appendix 6. Group interview guide for the immigrant-host family in New Zealand.

Appendix 7. Information sheet for the visiting friends/visiting relatives in the Philippines.

Appendix 8. Individual interview guide for the visiting relatives/visiting friends in the Philippines.

Appendix 9. Group interview guide for the visiting friends/visiting relatives in the Philippines. 


\section{Appendix 1. E-mail permission to the internet community moderator.}

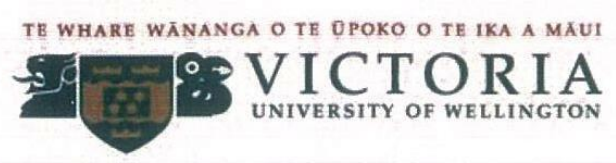

E-MAIL PERMISSION TO THE INTERNET COMMUNITY MODERATOR

Dear [Internet Community Moderator],

My name is Robert Capistrano and I am a Doctor of Philosophy (PhD) candidate in Tourism Management at Victoria University of Wellington (VUW). As a member of [Internet group name], I would like to ask permission to post the announcement attached to this request within our online Filipino community group. I would like to contact New Zealand-based Filipinos within our online community to canvass for participants in my doctoral study which is entitled, "Host-Guest Interactions: First-Generation Filipino Migrants in New Zealand and their Visiting Friends and Relatives (VFR) from the Philippines."

As a doctoral candidate in Tourism Management at VUW, I have discovered that host-guest social interactions in the context of migrants and their visiting friends or relatives are understudied. The aim of my project is to find out more about the social interactions between New Zealand's Filipino migrants and their visiting friends and relatives from the Philippines. I will need to interview several first-generation Filipino families in New Zealand who have had previous experience hosting visiting friends or relatives from the Philippines.

It is now a requirement for all university research involving people to be approved by the Victoria University of Wellington's Human Ethics Committee and this study has received this approval.

If you require further information about the study, please contact myself or my supervisors. We will gladly answer your questions.

Thank you very much for your continued support.

Truly yours,

Robert Charles Capistrano

Doctoral Candidate

School of Management

Victoria University of Wellington

Room 102, Rutherford House

23 Lambton Quay, Pipitea Campus,

Wellington, New Zealand 6011

E-mail: robert.capistrano@vuw.ac.nz

Phone No. +64-4-463 5233 ext. 8912

Mobile No. +64 220742193

Supervisors:

\section{Dr. Adam Weaver}

Senior Lecturer in Tourism Management

School of Management

Victoria University of Wellington

Room 917, Rutherford House

23 Lambton Quay, Pipitea Campus,

Wellington, New Zealand 6011

E-mail: adam.weaver@vuw.ac.nz

Phone No. +64-4- 4635375

Fax No. +64-4-463 5180
Philippine residence

(from October 2012- February 2013):

7 Natividad Street

San Francisco del Monte

Quezon City, Philippines 1105

Residence Phone No. +63-2-373 5863
Prof. Douglas Pearce

Professor of Tourism Management

School of Management

Victoria University of Wellington

Room 921, Rutherford House

23 Lambton Quay, Pipitea Campus,

Wellington, New Zealand 6011

E-mail: douglas.pearce@vuw.ac.nz

Phone No. +64-4- 4635715

Fax No. +64-4-463 5180 
Appendix 2. E-mail announcement to the internet community.

TE WHARE WANANGA O TE DPOKO O TE IKA A MAUI

E-MAIL ANNOUNCEMENT TO THE INTERNET COMMUNITY

Dear [Internet Community Group],

Magandang araw po. My name is Robert Capistrano, a current Doctor of Philosophy (PhD) candidate in Tourism Management at Victoria University of Wellington. I am contacting New Zealand-based Filipinos within the internet community to canvass for participants in my doctoral study which is' entitled, "Host-Guest Interactions: First-Generation Filipino Migrants in New Zealand and their Visiting Friends and Relatives (VFR) from the Philippines." My interest in VFR travel studies stems from being a Filipino with the majority of my relatives and friends residing in the United States and Canada, including my mother who lives in San Francisco, California for the last decade.

I have been in contact with [name of internet moderator] and s/he has permitted me to post this announcement within our Filipino online community. The [name of internet community] and [name of internet moderator] are not conducting this research and will have no access to data from this study. This is part of my PhD project on VFR travel studies and is independent from [name of internet community]. It is now a requirement for all university research involving people to be approved by the Victoria University of Wellington's Human Ethics Committee and this study has received this approval.

I am interested in examining the experiences of Filipino migrants here in New Zealand with respect to their social interactions with their visiting friends and/or relatives, and vice versa. Hence, I am seeking participants in my study that examines their social interactions.

In particular, first-generation Filipino migrants should meet all the following criteria:

- Former residents of the Philippines;

- New Zealand permanent residents or those who have acquired New Zealand citizenship

- Those who have hosted a Filipino friend or relative whose visit was made within the three to twelve months; and

- Can participate in both individual and family interviews between July and September 2012 in their New Zealand residence. Only family members who are at least 18 years old will be invited for the interviews. The individual interview which will take 60 to 90 minutes while the family interview will take no more than 60 minutes but may vary depending on how much information you provide

Your visiting friend or relative should meet all the following criteria:

- A Filipino citizen;

- At least 18 years old;

- Must have travelled to New Zealand and visited you within the last three to twelve months;

- Must only be carrying a visitor visa at the time of the trip;

- Currently residing in the Philippines; and

- Can participate in an individual interview which should take 60 to 90 minutes in their Philippine residence between November and December 2012. If your visiting friend or relative took the previous trip to New Zealand with a companion, a family or group interview will also be arranged which may last up to 60 minutes (if a group visited)

Should both you and your visiting friend or relative meet all of the criteria, kindly contact me directly either by phone or email: robert.capistrano@vuw.ac.nz and I will provide you with information to discuss the research in further detail. Kindly do not respond to the entire group but to me only.

I will send further information once I have received your much appreciated response of willingness to participate. I wish to thank you for your kind consideration of assistance in helping my research and study.

Salamat po,

Robert Charles Capistrano PhD Candidate

Victoria University of Wellington

School of Management

E-mail: robert.capistrano@vuw.ac.nz

Mobile No.: +64 220742193 


\section{Appendix 3. Information sheet for the immigrant-host family interviews in New Zealand.}

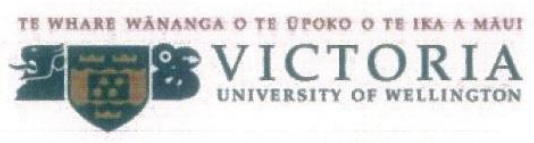

\section{INFORMATION SHEET FOR THE MIGRANT-HOST FAMILY INTERVIEWS IN NEW ZEALAND}

Dear [Name of Research Participant]:

I would like to thank you for expressing interest in my study. As a doctoral candidate in Tourism Management at Victoria University of Wellington, I have discovered that host-guest social interactions in the context of migrants and their visiting friends or relatives are understudied. The aim of my project is to find out more about your social interactions with your visiting friend(s)/relative(s) that encompasses the pre-, during, and post-trip experiences. I wish to analyze how the social interactions between you, your family, and your guest(s) influence the experiences that you share. For this, I will need to interview several first-generation Filipino families in New Zealand who have had previous experience hosting visiting friends or relatives from the Philippines.

I wish to interview all family members of the hosting household who are at least 18 years old. The interviews are composed of one-on-one interviews with the individual family members followed by a group interview (if you hosted as a group).

Members of your family will be requested to:

- Participate in both individual and group interviews at some point between July and September 2012. The individual interviews will take 60 to 90 minutes. The group interview should take no more than 60 minutes. The duration of the interviews may vary depending on how much information you provide.

- Assist in arranging for an interview with your respective visiting friend(s) or relative(s) in the Philippines between October and December 2012.

- Members of your family are welcome to bring souvenirs and photos to the interview as reminders of your interactions with your guest(s)

After the winter season, I will be going home to the Philippines so that I can interview your respective guest(s). As I am examining host-guest social interactions, I would like to ensure that both your family and your respective visiting friend(s)/relative(s) from the Philippines are willing to be interviewed. Otherwise, I will not be able to properly conduct my study.

I have included some guidelines below which I will be following in the conduct of this research:

(i) Respect for Privacy Rights of the Participants

- I will make every effort to maintain your anonymity to both strangers and common acquaintances. In this study, you will be identified by your significant role in the family (i.e., mother, father, son, daughter). Pseudonyms will also be used. 
- While I am aware that you will not be named in the research, specific examples and anecdotes that you provide could make you identifiable to other people-specifically family members and/or friends.

- I wish to tape record our conversations so that I have an accurate account of our discussions. If you do not wish to be tape recorded, I would like your permission to take notes during the interviews instead.

- In terms of the shared information resulting from the interview session, only you will be able to check the transcripts of your particular individual interview and the group interview that you may participate in.

- With regard to information within the family or group interview, you and your family can choose to keep this information confidential.

- Your guest(s) from the Philippines will be unable to read the transcripts from our conversation(s).

- I may request your permission to use a reproduction of one your photos in the study. However, photographs having identifiable features attributable to you will be obscured or pixelated. Any further use of the information will require your written consent. I may also ask to photograph one or more of your souvenirs for this study. This photograph may appear in the study or any published article.

(ii) Withdrawal of Information

- As an added assurance to your comfort level, I want you to know that you are free to withdraw on or before 15 December $\mathbf{2 0 1 2}$ without providing reasons. Should you decide to withdraw from this research, all information related to you will be destroyed.

(iii) Transparency

- The information you will provide will be used for this research in the form a PhD thesis and possible academic journal and conference papers.

- A summary of the research findings will be available to you by the end of this project via e-mail by about February 2014.

- The interview notes and tapes will be destroyed two years after completion of this project (approximately 2016).

It is now a requirement for all university research involving people to be approved by the Victoria University of Wellington's Human Ethics Committee and this study has received this approval. 
Filipino migrant families and their guests may find it a worthwhile experience to share their experiences, culture and values. A separate information sheet addressed to your respective guest will help explain the purpose of my study and some further instructions. Feel free to forward the attached information sheet to your relative/friend about this study.

If you require further information about the study, please contact myself or my supervisors. We will gladly answer your questions. Thank you for your interest.

Truly yours,

\section{Robert Charles Capistrano}

Doctoral Candidate

School of Management

Victoria University of Wellington

Room 102, Rutherford House

23 Lambton Quay, Pipitea Campus,

Wellington, New Zealand 6011

E-mail: robert.capistrano@vuw.ac.nz

Phone No. +64-4-463 5233 ext. 8912

Mobile No. +64 220742193

\section{Supervisors:}

\section{Dr. Adam Weaver}

Senior Lecturer in Tourism Management

School of Management

Victoria University of Wellington

Room 917, Rutherford House

23 Lambton Quay, Pipitea Campus,

Wellington, New Zealand 6011

E-mail:adam.weaver@vuw.ac.nz

Phone No. +64-4- 4635375

Fax No. +64-4-463 5180

\section{Philippine residence}

(from October 2012- February 2013):

7 Natividad Street

San Francisco del Monte

Quezon City, Philippines 1105

Residence Phone No. +63-2-373 5863
Prof. Douglas Pearce

Professor of Tourism Management

School of Management

Victoria University of Wellington

Room 921, Rutherford House

23 Lambton Quay, Pipitea Campus,

Wellington, New Zealand 6011

E-mail: douglas.pearce@vuw.ac.nz

Phone No. +64-4- 4635715

Fax No. +64-4-463 5180 


\section{Appendix 4. Immigrant-host family in New Zealand/visiting friends and relatives in} the Philippines Consent Form.

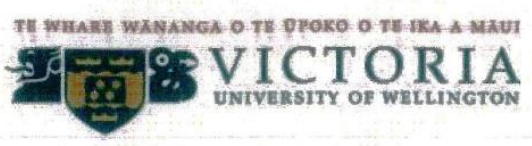

MIGRANT-HOST FAMILY IN NEW ZEALAND / VISTING FRIENDS AND RELATIVES IN THE PHILIPPINES INTERVIEW CONSENT FORM

Thesis Title: Host-Guest Interactions: First-Generation Filipino Migrants in New Zealand and their Visiting Friends and Relatives (VFR) from the Philippines

This document confirms that I was completely briefed about this research study and the circumstances of my participation as a respondent. I was given the opportunity to clarify issues, and I was satisfied with the answers and clarifications made by the researcher, hence, I am giving my consent, free from any forms of pressure and intimidation.

It is my understanding that I may withdraw myself from this study during the administration of this interview and inform the researcher of my withdrawal no later than 15 December $\mathbf{2 0 1 2}$. Should I decide to withdraw from this study, I do not have to provide a reason for such withdrawal. All information that pertains to me will be destroyed.

Please confirm your consent to participate in this research by placing a tick in the relevant boxes below:

- I have been provided a copy of the Information Sheet for the Migrant-Host Family /Visiting friends \& relatives [please encircle one].

I confirm that I was briefed about the nature and objectives of this study.

$\square \quad$ I understand that I will be interviewed as part of this research. I am able to participate in the following: $\square$ Individual interview

$\square$ Group interview, if necessary (if more than one host/guest)

I understand that depending on how much information I provide, the individual interview may take 60 to 90 minutes while the group interview should take no more than 60 minutes.

ㅁ I understand that I have the right to check the transcripts regarding my own private interview and the group interview that I participated in. However, I understand that I will be unable to review the interview transcripts of other household members and my respective guest(s) from the Philippines/host(s) from New Zealand.

$\square \quad$ I understand that my family and I can decide to keep information shared within the family or group interview confidential.

ㅁ I may decide not to provide answer(s) to certain question(s) without giving a reason.

$\square \quad$ I understand that the interviews will be tape recorded for an accurate account of discussions.

I do not wish to be tape recorded but consent to having notes taken during the interview(s).

I understand that my name will not be included in the written report/research, in which case, a pseudonym will be used.

․ I understand that while I am aware that I will not be named in the research, specific examples and anecdotes that I provide could make me identifiable to other people-specifically family members and friends.

$\square \quad$ I understand that the researcher may ask permission to use a reproduction of one of my photos in the study. I understand that any identifiable features attributable to me will be obscured or pixelated. 
I understand that the research may ask permission to photograph one or more of my souvenirs for his study. This photograph may appear in the researcher's thesis or in a published article.

$\square \quad$ I understand that the information I have provided will be used only for this research in the form of a PhD thesis and possible academic journal and conference papers. Any further use of the information will require my written consent.

- I understand that the interview notes and tapes will be destroyed two years after completion of this project (approximately 2016).

I wish to receive a summary of the research findings which will be available to by the end of this project in about February 2014 through [please choose one]: $\square$ E-mail $\square$ Post mail

Printed Name:

Mailing address:

E-mail address:

Signature:

Date:

Consent Form Prepared by:

Researcher:

Degree:

Robert Charles G. Capistrano

Doctor of Philosophy in Tourism Management

School of Management

Victoria University of Wellington

Room 102, Rutherford House

23 Lambton Quay, Pipitea Campus

E-mail:

Wellington, New Zealand 6140

Mobile No.:

robert.capistrano@vuw.ac.nz

+64 220742193 (New Zealand)

+632 3735863 (Philippine residence)

Supervisors:

\section{Dr. Adam Weaver}

Senior Lecturer in Tourism Management School of Management

Victoria University of Wellington

Room 917, Rutherford House

23 Lambton Quay, Pipitea Campus,

Wellington, New Zealand 6011

E-mail:adam.weaver@vuw.ac.nz

Phone No. +64-4- 4635375

Fax No. +64-4-463 5180
Prof. Douglas Pearce

Professor of Tourism Management

School of Management

Victoria University of Wellington

Room 921, Rutherford House

23 Lambton Quay, Pipitea Campus,

Wellington, New Zealand 6011

E-mail: douglas.pearce@vuw.ac.nz

Phone No. +64-4- 4635715

Fax No. +64-4-463 5180 


\title{
Appendix 5. Individual interview guide for the immigrant-host family in New Zealand.
}

\author{
Individual Interview Guide for the Migrant-Host Family in New Zealand
}

I will be asking probing questions throughout the interview in order to elicit more detailed responses such as:

What exactly did you mean by that?

you?

How do you feel about that?

Why is that important to

Can you give me an example?

The migrant-hosts are being asked about a visit by their guest(s) from the Philippines, who are either relative(s) or friend(s).

\section{Pre-trip Phase}

1. If relatives, how are you and your guest(s) related? If friends, how did you become friends with your guest(s)?

2. Do you consider yourself a host? What activities do you associate with hosting?

3. Did you communicate with your guest(s) as the trip was being planned? How did you and your guest(s) communicate? What did you discuss?

4. Why did you host your guest(s)? What is your primary motivation for hosting your guest(s)?

5. Do you believe that your guest(s) would visit New Zealand if you did not live here?

6. What was/were your expectation(s) in the anticipation of your guest ('s/s') visit?

7. What assistance (if any) did you provide your guest(s) before their visit (i.e., airfare; visa application, etc.)?

8. Did you have any difficulties in preparing for your guest $\left(' s / s^{\prime}\right)$ visit? What were your difficulties, if any?

9. Do you appreciate visits from guest(s) in your new homeland?

\section{During-Trip Phase}

1. How long was your guest('s/s') visit? Is this a repeat visit for them?

2. If you accommodated your guest in your home, please describe the experience. If you did not accommodate your guest in the home, why was that the case?

3. Did your guest(s) bring any gifts/items during the visit? If so, what did they bring?

4. What are the most memorable aspects of the hosting experience? What do you think was your best time together with your guest(s) in New Zealand?

5. What obligations do you have when hosting your guest(s)? What duties did you perform together?

6. Can you give me examples of hosting activities that you did enjoy and did not enjoy? Why did you or did not enjoy them?

7. How do you think Filipino norms and values influence your hosting of your guest(s)? Do you think that your hosting behaviour reflects aspects of Filipino culture and/or Kiwi culture?

8. What places did you visit with your guest(s)? What places did your guest(s) visit unescorted?

9. How do you consider and incorporate pakikipagkapwa (humaneness) when dealing with your guest(s)? 
Appendix 6. Group interview guide for the immigrant-host family in New Zealand.

Group Interview Guide for the Migrant-Host Family in New Zealand

I will be asking probing questions throughout the interview in order to elicit more detailed responses such as:

What exactly did you mean by that? How do you feel about that?
Why is that important to you? Can you give me an example?

1. Why do you consider yourselves hosts? What activities do you associate with hosting?

2. As a family, what was/were your motivation or reasons for hosting? Why do you want your friend(s)/relative(s) to visit?

3. Did your family plan and prepare together in anticipation of your guest ('s/s') visit?

4. What were your family's expectations in hosting? What do you expect from your guest(s)?

5. If you accommodated your guest(s) in your home, how would your family describe the experience? If your guest(s) was not accommodated in your home, how did you feel about that?

6. How was the selection of activities being decided? Did the guest(s) have some input?

7. Can you give me examples of activities that you enjoyed doing as a family together with your guest(s)? (everyone can give examples to compile a list and make use of the photos, if there are some)

8. What do you think is the ideal length of the visit? Can the visit be too long or can it be too short? Or does it matter?

9. As a host family, what do you think are your obligations to your guest(s)? Where you able to fulfill them?

10. Does every family member enjoy hosting? As a family can you agree on a time that you did (or did not) enjoy hosting? Why was that particular experience enjoyable/not enjoyable?

11. As a family, can you think of any advice on how to better organize the next visit of your guest(s)?

12. As a host family, do you think New Zealand offers experiences or attractions marketed to your guest(s)?

13. What are the most memorable activities of your family's hosting experience? As a group, what was your most memorable shared activity with your guest(s)?

14. As a family, what Filipino norms, values and practices do you think you still share with your guest?

15. What Filipino norms, values and practices, if any, have you let go of since moving to New Zealand?

16. Does your family believe that hosting strengthens your social and cultural ties with your guest(s)?

17. Do you feel that pakikipagpalagayang-loob (level of mutual trust) still exist between your family and your guest(s)?

18. Did your family feel a greater sense of belonging in New Zealand as a result of hosting?

19. Does the visit of your guest(s) promote the values and traditions of your former homeland or the values and traditions of your new homeland? How?

20. As a family, how important was it for you to have your guest(s) visit you? 


\section{Appendix 7. Information sheet for the visiting friends/visiting relatives in the Philippines.}

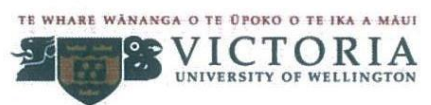

INFORMATION SHEET FOR THE VISITING FRIENDS/VISITING RELATIVES IN THE PHILIPPINES

Dear [Name of Research Participant]:

Magandang araw po mula sa New Zealand. My name is Bob Capistrano, a current Doctor of Philosophy (PhD) candidate in Tourism Management at Victoria University of Wellington. The family of Mr./Ms./Mrs. your friend/relative, has expressed their interest to participate in my study, entitled "Host-Guest Interactions: First-Generation Filipino Migrants in New Zealand and their Visiting Friends and Relatives (VFRs) from the Philippines." They have given your name to me as a prospective participant for my study.

My interest in VFR travel studies stems from being a Filipino citizen with the majority of my relatives and friends residing in the United States and Canada, including my mother who has lived in San Francisco, California for the last decade. As a doctoral candidate at Victoria University of Wellington, I have discovered that the host-guest social interactions in the context of migrants and their visiting friends or relatives are understudied.

The aim of my project is to find out more about your social interactions with your host family in New Zealand encompassing your pre-, during, and post-trip experiences. I wish to analyze how the social interactions between you and your host influence the experiences that you share. For this, I will need to interview several first-generation Filipino families in New Zealand and their previous guests after they have returned to the Philippines.

Mr./Ms./Mrs.

referred me to you because you meet all the following criteria: you have visited New Zealand within the last 3 to 12 months, you have been hosted by a Filipino family, you are at least 18 years old, and you currently reside in the Philippines. I am going home to the Philippines during the last quarter of this year and I am looking forward to meeting and interviewing you between October and December 2012 . The one-on-one interview should take 60 to 90 minutes and take place in your residence. If you took your previous trip to New Zealand with companions, I would also like to interview you as a group which may last up to 60 minutes (if a group of you visited). The duration of both interviews may vary depending on how much information you provide. You are welcome to bring photos and souvenirs to the interviews.

As I am examining host-guest social interactions, I will first conduct an interview with your host family in New Zealand between July and September 2012. Afterwards, I would like to ensure that you are free and willing to be interviewed during the last quarter of 2012 in your Philippine residence. Otherwise, I will not be able to properly conduct my study.

I have included some guidelines below which I will be following in the conduct of this research:

- I will make every effort to maintain your anonymity to both strangers and common acquaintances. In this study, you will be identified by your significant role in the family (i.e., visiting relative; visiting friend). Pseudonyms will also be used.

- While I am aware that you will not be named in the research, specific examples and anecdotes that you provide could make you identifiable to other people - specifically family members and friends.

- I wish to tape record our conversations so that I have an accurate account of our discussions. If you do not wish to be tape recorded, I would like your permission to take notes during the interviews instead.

- In terms of the shared information resulting from the interview session, only you will be able to check the transcripts of your particular individual interview and the group interview that you will participate in. 
- With regard to information within the family or group interview, you and your family can choose to keep this information confidential.

- Your host from New Zealand will be unable to read the transcripts from our conversation(s).

- I may request for your permission to use a reproduction of one your photos in the study. However, photographs having identifiable features attributable to you will be obscured or pixelated. Any further use of the information will require your written consent. I may ask to photograph one or more of your souvenirs for this study. This photograph may appear in the study or any published article.

(ii)

Withdrawal of Information

- As an added assurance to your comfort level, I want you to know that you are free to withdraw on or before 15 December 2012 without providing reasons. Should you decide to withdraw from this research, all information related to you will be destroyed.

(iii) Transparency

- The information you will provide will be used for this research in the form a PhD thesis and possible academic journal and conference papers.

- A summary of the research findings will be available to you by the end of this project via e-mail by about February 2014.

- The interview notes and tapes will be destroyed two years after completion of this project (approximately 2016)

It is now a requirement for all university research involving people to be approved by the Victoria University of Wellington's Human Ethics Committee and this study has received this approval.

As previous visiting friend(s) from the Philippines, I hope that you will find it to be a worthwhile experience to share your experiences and our culture and values and I hope that you find it to be the case. If you require further information about the study, please contact myself or my supervisors. We will gladly answer your questions. Thank you for your interest.

Truly yours,

Robert Charles Capistrano

Doctoral Candidate

School of Management

Victoria University of Wellington

Room 102, Rutherford House

23 Lambton Quay, Pipitea Campus,

Wellington, New Zealand 6011

E-mail: robert.capistrano@vuw.ac.nz

Phone No. +64-4-463-5233 ext. 8912

Mobile No. +64-22-07 42193

Supervisors:

Dr. Adam Weaver

Senior Lecturer in Tourism Management

School of Management, Victoria University of Wellington

Room 917, Rutherford House

23 Lambton Quay, Pipitea Campus,

Wellington, New Zealand 601

E-mail: adam.weaver@vuw.ac.nz

Phone No. +64-4- 4635375

Fax No. +64-4-463 5180
Philippine residence

(from October 2012- February 2013):

7 Natividad Stree

San Francisco del Monte

Quezon City, Philippines 1105

Residence Phone No. (+632) 3735863

Prof. Douglas Pearce

Professor of Tourism Management

School of Management, Victoria University of Wellington Room 921, Rutherford House

23 Lambton Quay, Pipitea Campus,

Wellington, New Zealand 6011

E-mail: douglas.pearce@vuw.ac.nz

Phone No. +64-4- 4635715

Fax No. +64-4-463 5180 


\title{
Appendix 8. Individual interview guide for the visiting relatives/visiting friends in the Philippines.
}

\author{
Individual Interview Guide for the Visiting Relatives/Visiting Friends in the Philippines
}

I will be asking probing questions throughout the interview in order to elicit more detailed responses such as:

\author{
What exactly did you mean by that? \\ Why is that important to
}

you?

How do you feel about that?

Can you give me an example?

The visiting relative(s) are being asked about a visit they made to their hosts in New Zealand.

\section{Pre-trip Phase}

1. How are you and your host related? / For VFs: How did you become friends with your host?

2. Did you communicate with your host as the trip was being planned? How did you and your host communicate? What did you discuss?

3. Why did you visit your host? What was the primary motivation for the trip?

4. Do you believe that you would have visited New Zealand if your host did not live there?

5. What was/were your expectation(s) in the anticipation of your visit?

6. What assistance (if any) did your relative(s) provide before your visit? (i.e., airfare; visa application, etc.)?

7. Did you have any difficulties in preparing for the visit?

8. Do you consider yourself as a guest? Why or why not?

\section{During-Trip Phase}

1. How long was your visit? Is this a repeat visit for you?

2. What type of accommodation did you use during the visit? Please describe the experience of staying with your host (or using other forms of accommodation).

3. Did you bring any gifts/items during the visit? If so, what did you bring?

4. What are the most memorable aspects of your visit? What do you think was your best time together with your host in New Zealand?

5. What obligations do you have when you visit your relative? What duties did you perform together?

6. Can you give me examples of the activities that you did enjoy and did not enjoy? Why did you enjoy or did not enjoy them?

7. How do you think Filipino norms and values influence your visit to your host? Do you think that the behaviour of your host reflects aspects of Filipino culture and/or Kiwi culture?

8. What places did you visit with your host? What places did your visit unescorted?

9. Do you have difficulties in terms of pakikisama (transaction; civility) to your host?

10. How do you consider and incorporate pakikipagkapwa (humaneness) when dealing with your host?

11. Did the visit lead to any misunderstandings in terms of your relationship with your host?

12. Do you feel New Zealand offers experiences or attractions that are marketed to you as a Filipino? Why or why not?

13. How important was it for you to visit your relative(s)? 


\section{Post-trip Phase}

1. Looking back, how would you describe your travel experience? Was it successful or not?

2. How would you describe the closeness of your relationship with your relative(s)/friend(s)?

3. Did the visit re-establish/renew the relationship that you have with your relative(s)/friend(s)?

4. Did the visit strengthen your social and cultural ties with your relative(s)/friend(s)?

5. Do you feel that your relative('s/s')/ /friend('s/s') behaviour still reflects their Filipino culture \& values? Why or why not?

6. What did you learn about New Zealand during the course of your visit?

7. Would you recommend New Zealand as a tourist destination to other Filipinos?

8. What would be your motivation(s) or reason(s) should you decide to visit your relative(s)/friend(s) again?

9. Do you think you will be hosted by your relative/friend in New Zealand in the near future?

10. What are your obligations (if any) to your host after the trip?

11. How are you continuing to communicate with your relative(s)/friend(s) after the trip?

12. Since you have returned home, how do you intend to reciprocate the hospitality showed by your host?

13. Do you believe that visiting your host makes you feel in solidarity with them (pakikipagpalagayang-loob)? If yes, how so?

14. As a visitor, is there anything else you would like to share with me? 


\title{
Appendix 9. Group interview guide for the visiting friends/visiting relatives in the Philippines.
}

\section{Group Interview Guide for the Visiting Relatives/Visiting Friends in the Philippines}

I will be asking probing questions throughout the interview in order to elicit more detailed responses such as:

What exactly did you mean by that?

How do you feel about that?

\author{
Why is that important to you? \\ Can you give me an example?
}

1. As a family/group, what was/were your motivation or reasons for visiting?

2. Did your family/group plan and prepare together in the anticipation of your visit?

3. What were your family's/group's expectations in visiting? What were you expecting from your host?

4. Did you stay with your host? If so, how did you feel about that? If not, how did you feel about that?

5. Did your family have some input into the selection of activities?

6. Can you give me examples of activities that you enjoyed doing in New Zealand as a family/group together with your host? (everyone can give examples to compile a list and make use of the photos, if there are some)

7. What do you think is the ideal length of the visit? Can the visit be too long or can it be too short? Or does it matter?

8. What are your obligations to your host? Were you able to fulfill them?

9. Does every family/group member enjoy the visit? As a family/group can you agree on a time that you did (or did not) enjoy the visit? Why was that particular experience enjoyable/not enjoyable?

10. As a family/group, can you think of any advice on how to better organize the next visit?

11. As a family/group, do you think New Zealand offers experiences or attractions marketed to you?

12. What are the most memorable aspects of your family's/group's visiting experience? As a family/group, what was your most memorable shared activity with your host?

13. As a family/group, what Filipino norms, values and practices do you think you still share with your host?

14. Does your family/group believe that visiting strengthens your social and cultural ties with your host?

15. Do you feel that pakikipagpalagayang-loob (level of mutual trust) still exist between your family/group and your host?

16. Did your family/group feel a greater sense of belonging and solidarity with your host while you were in New Zealand as a result of the visit?

17. Did the visit promote and allow you to share Filipino values and traditions with your host? Why or why not?

18. As a family/group, how important was it for you to visit your host? 